\title{
Synthesis of the elements in stars: forty years of progress
}

George Wallerstein

Department of Astronomy, University of Washington, Seattle, Washington 98195

Icko Iben, Jr.

University of Illinois, 1002 West Green Street, Urbana, Illinois 61801

Peter Parker

Yale University, New Haven, Connecticut 06520-8124

Ann Merchant Boesgaard

Institute for Astronomy, 2680 Woodlawn Drive, Honolulu, Hawaii 96822

Gerald M. Hale

Los Alamos National Laboratory, Los Alamos, New Mexico 87544

Arthur E. Champagne

University of North Carolina at Chapel Hill, Chapel Hill, North Carolina 27594

and Triangle Universities Nuclear Laboratory, Duke University, Durham, North Carolina

27706

Charles A. Barnes

California Institute of Technology, Pasadena, California 91125

Franz Käppeler

Forschungzentrum, Karlsruhe, D-76021, Germany

Verne V. Smith

University of Texas at El Paso, El Paso, Texas 79968-0515

Robert D. Hoffman

Steward Observatory, University of Arizona, Tucson, Arizona 85721

Frank X. Timmes

University of California at Santa Cruz, California 95064

Chris Sneden

University of Texas, Austin, Texas 78712

Richard N. Boyd

Ohio State University, Columbus, Ohio 43210

Bradley S. Meyer

Clemson University, Clemson, South Carolina 29630

David L. Lambert

University of Texas, Austin, Texas 78712

(Received 25 June 1997)

Forty years ago Burbidge, Burbidge, Fowler, and Hoyle combined what we would now call fragmentary evidence from nuclear physics, stellar evolution and the abundances of elements and isotopes in the solar system as well as a few stars into a synthesis of remarkable ingenuity. Their review provided a foundation for forty years of research in all of the aspects of low energy nuclear experiments and theory, stellar modeling over a wide range of mass and composition, and abundance studies of many hundreds of stars, many of which have shown distinct evidence of the processes suggested by $\mathrm{B}^{2} \mathrm{FH}$. In this review we summarize progress in each of these fields with emphasis on the most recent developments. [S0034-6861(97)00204-3] 


\section{CONTENTS}

I. Preface by E. Margaret Burbidge, Geoffrey R. Burbidge, and Fred Hoyle

II. Introduction by George Wallerstein

A. The cosmological foundations of $\mathrm{B}^{2} \mathrm{FH}$

B. The astronomical background in 1957

C. The eight processes

1. Hydrogen burning

2. Helium burning

3. The $\alpha$ process

4. The $e$ process

5. The $s$ process

6. The $r$ process

7. The $p$ process

8. The $x$ process

D. Neutrino astrophysics

1. The solar neutrino problem

2. Other aspects of neutrino astrophysics

E. Related reviews

III. Stellar Evolution by Icko Iben, Jr.

A. Historical preliminary

B. Evolution of single stars that become white dwarfs

1. Overview

2. Nucleosynthesis and dredge-up prior to the AGB phase

3. Nucleosynthesis and dredge-up during the AGB phase

4. The born-again AGB phenomenon

5. Other mixing processes, and wind mass loss, which affect surface composition

C. Evolution of massive single stars that produce neutron stars or black holes

D. Close binary star evolution

1. Modes of mass transfer and of orbital angular momentum loss

2. Scenario modeling

a. Cataclysmic variables and novae

b. White dwarf mergers: R CrB stars and type Ia supernovae

c. X ray binaries and pulsars

IV. Hydrogen Burning in the $p p$ Chain and $\mathrm{CN}$ Cycle by Peter Parker

A. The $p\left(p, e^{+} \nu_{e}\right) d$ reaction

B. The ${ }^{3} \mathrm{He}\left({ }^{3} \mathrm{He}, 2 p\right){ }^{4} \mathrm{He}$ reaction

C. The ${ }^{3} \mathrm{He}(\alpha, \gamma){ }^{7} \mathrm{Be}$ reaction

D. The ${ }^{7} \mathrm{Be}(\mathrm{p}, \gamma){ }^{8} \mathrm{~B}$ reaction

E. The ${ }^{7} \mathrm{Li}(n, \gamma){ }^{8} \mathrm{Li}$ reaction

F. The ${ }^{14} \mathrm{~N}(p, \gamma){ }^{15} \mathrm{O}$ reaction

G. The ${ }^{17} \mathrm{O}(p, \alpha){ }^{14} \mathrm{~N}$ reaction

H. The Hot CNO cycle

V. The $x$ Process by Ann Merchant Boesgaard

A. Introduction and retrospective

B. Abundances

1. Lithium

2. Beryllium

3. Boron

C. Nonlocal thermodynamic-equilibrium effects

D. Production mechanisms

1. Big Bang

2. Spallation

3. Asymptotic giant branch stars

4. Supernovae

VI. Helium Burning by Gerald M. Hale
A. Triple- $\alpha$ capture 1020

B. $\alpha+{ }^{12} \mathrm{C}$ capture 1021

1. E1 capture 1021

a. Direct measurements 1021

b. $\beta$-delayed $\alpha$ spectrum from the decay of ${ }^{16} \mathrm{~N}$

1022

2. E2 capture 1022

3. Other analyses and recommended values 1023

VII. H Burning in the NeSi Region: Laboratory Studies by Arthur E. Champagne

A. Introduction

1024

1024

B. Experimental approaches

1024

C. The neon-sodium cycle at low temperatures 1025

1. Reaction rates 1025

2. Network calculations

D. The magnesium-aluminum cycle at low temperatures

1. Reaction rates

2. Network calculations

1026

999

1000

1000

1000

1001

1001

1001

1001

1001

1003

1004

1006

1007

1007

1009

1009

1009

1010

1010

1012

1013

1013

1013

1014

1015

1015

1015

1016

1016

1016

1016

1016

1017

1018

1018

1019

1019

1019

1019

1019

1020

1020

E. High-temperature behavior

1. Reaction rates

2. Network calculations

F. Conclusion

VIII. Observational Evidence of Hydrogen Burning by George Wallerstein

A. CN cycle and mixing

B. O depletion and the enhancement of $\mathrm{Na}$ and $\mathrm{Al}$

C. Origin of the $\mathrm{Na}$ and $\mathrm{Al}$ enhancements

IX. Carbon, Neon, Oxygen, and Silicon Burning by Charles A. Barnes

A. Introduction

B. Carbon burning

C. Neon burning

D. Oxygen burning

E. Silicon burning

X. $s$ Process: Laboratory Studies and Stellar Models by Franz Käppeler

A. The $s$ process since $\mathrm{B}^{2} \mathrm{FH}$

B. Laboratory experiments

1. Neutron capture cross sections

2. Stellar $\beta$ decay

C. The canonical $s$ process

1. The $\sigma \mathrm{N}$ curve

2. Branchings

XI. Observations of the $s$ Process by Verne V. Smith

A. Brief history

B. Observations of nucleosynthesis and mixing in $\mathrm{CH}, \mathrm{Ba}, \mathrm{S}$, and $\mathrm{C}$ stars

C. The $s$ process as a function of metallicity

D. Rubidium and the $s$-process neutron density

E. Recent models: Radiative burning of ${ }^{13} \mathrm{C}$ during the AGB interpulse phase

1027

1027

1028

1029

1029

1029

1030

1030

1031

1032

1033

1034

1034

1035

1036

1036

1036

1038

1038

1038

1038

1039

1040

1040

1041

1042

1042

1043

1043

1045

1046

XII. The $r$ Process by Robert D. Hoffman and Frank X. Timmes

1047

A. Introduction

1047

B. A search for the astrophysical site

1047

C. Early model results, from conflict to clarity 1048

D. Twisting in the wind 1049

E. Concluding remarks

XIII. Observations of the $r$ Process by Chris Sneden

1049

1050

A. Defining the $r$-process elements 1050

B. Early $r$-process discoveries 1051

C. Recent $r$-process surveys 1051

D. Thorium and the age of the halo and disk 1053

E. Filling out the picture 1054

XIV. The $p$ Process by Richard N. Boyd 1054 

A. The $p$ process
B. Early $p$-process models
C. The $\gamma$ process
D. The $r p$ process
E. The $\nu$ process
F. Recent developments
G. Summary

$\mathrm{XV}$. The $e$ Process and the Iron-Group Nuclei by Bradley S. Meyer

A. Energetics and equilibria

B. Statistical equilibrium

C. A brief history of the ideas of iron-group element synthesis

D. Significance for astrophysics

XVI. Carbon Stars: Where Theory Meets Observations

by David L. Lambert

A. Prologue

B. Carbon stars-An observer's view

1. What is a carbon-rich star?

2. What makes a carbon-rich star?

3. The principal types of carbon-rich stars

a. R-type carbon stars

b. N-type carbon stars

C. Epilogue

c. Barium and related stars

XVII. Conclusions

Acknowledgments

References

\section{PREFACE}

It is curious that both the primordial and stellar theories of the origin of the elements should have been published in the same year, 1946. Little notice was at first taken of the stellar theory, attention being directed at first overwhelmingly to Gamow's suggestion of associating nucleosynthesis with the origin of the Universe.

While it is true that the one theory had to do with cosmology, the other did not. The suggestion that stellar nucleosynthesis had a connection to cosmology was an invention of Robert Oppenheimer and it never had any reality to it, since the first paper on stellar nucleosynthesis appeared in 1946, two years before the steady-state cosmological model was published. Nor were the objections to primordial synthesis by neutron addition by any means confined to the well-known gaps at $A=5$ and $A=8$, as is sometimes stated. There was always the pragmatic objection that the elements were distributed with too much spatial irregularity to be attributed to a universal origin. And the well-known iron peak was an obvious feature of solar system abundances, implying that at least in some places matter had been able to approach its most stable form.

For matter to approach its most stable form, temperatures in stellar interiors would be needed of the order of a hundred times those in main-sequence stars, a requirement that it would have been difficult to accept if the beginnings of an understanding of supernovae had not emerged in the early 1940s. In a rough kind of way it was possible to compare supernovae with ordinary stars in the same way that, in the mid 1940s, people were comparing nuclear weapons with chemical ones, this suggest- ing that temperatures vastly higher than those that seemed plausible in Eddington's day might be possible.

The fact that primordial nucleosynthesis ran far ahead of stellar nucleosynthesis in the years up to the early 1950s turned out to be advantageous to the eventual emergence of the $\mathrm{B}^{2} \mathrm{FH}$ paper in 1957 , because it permitted facts to accumulate quietly without any frenzied circus developing. In 1952, Salpeter discussed the stellar formation of carbon from alpha particles, and a few months later the relation of carbon synthesis to oxygen synthesis was shown to require a state in the carbon nucleus of about $7.65 \mathrm{MeV}$ above ground level. When this state was actually found, the laboratory discovery carried a strong measure of conviction for all those who were involved in it.

At the same time surveys of nearby stars by several groups, including some of us, were showing variations of metal abundances to hydrogen, the beginning of the concept of metallicity, that seemed impossible to associate with some form of universal synthesis. We were also puzzled by our 1953-54 analysis of high-dispersion spectra obtained at McDonald Observatory, which showed strange overabundances of some heavy elements and seemed to indicate that neutrons were involved. And in 1953-54, carbon-burning and oxygen-burning were found.

It was in the autumn of 1954 that the team of $\mathrm{B}^{2} \mathrm{FH}$ came together in the quiet ambience of Cambridge, England, without, as mentioned above, any frenzied circus developing.

During 1954-55 calculations involving neutron production in the interiors of evolved stars with heliumburning cores surrounded by a hydrogen-burning shell were followed by calculations on neutron addition to elements from neon to scandium. The process of neutron addition was slow enough for each neutron capture to be followed by beta decay (the origin of what we later named the $s$ process). In the same period, we became aware that A.G.W. Cameron was working along similar lines in Canada. These calculations showed the possibility of explaining the characteristic odd-even effect in abundance ratios.

In the autumn of 1955 we all moved, or returned, to Pasadena, where there was still a relatively peaceful setting at both the Kellogg Radiation Laboratory at Caltech and the Mount Wilson and Palomar offices at 813 Santa Barbara Street. We now also had the great advantage of being at the home of the large telescopes. In 1952 the process of neutron liberation and capture had been clinched by Paul Merrill's discovery of the unstable element technetium in S stars (evolved red giants), and we were able to obtain spectra with the Mount Wilson 100-inch telescope of an evolved star of the class known as "Barium II stars," in which we determined the overabundances of just those heavy elements that had at least one isotope with a magic neutron number.

One could not be in the same town as Walter Baade without hearing a lot about his famous light curve of the supernova in the galaxy IC 4182. With the interest of all 
four of us in supernovae as the spectacular death throes of stars at the end of their active evolution and thermonuclear energy production, we latched onto this. The exponential decay of the energy output of this supernova immediately suggested radioactive decay, following collapse, the release of a great burst of energy, neutrons, and neutrinos, and the formation of heavy unstable nuclei.

Also in 1956, an improved solar-system abundance curve became available from Suess and Urey. Values in the upper half of the chart of the nuclides showed two things: the association of magic neutron numbers with abundance peaks, and a separation of peaks corresponding to both slow and rapid neutron addition, the latter being fast enough not to allow time for the beta-decay involved in slow neutron addition. And released at that time were hitherto classified data on $(n, \gamma)$ cross sections for individual isotopes of elements, without which a meaningful analysis of what in $\mathrm{B}^{2} \mathrm{FH}$ was called the $s$ process would not have been possible.

$\mathrm{B}^{2} \mathrm{FH}$ can be seen in retrospect to have been a highly creative review article, putting together what the four authors had done previously, together with the facts on which the theory would now be based with considerable confidence. We each brought ideas and data from very different parts of physics to the table, and occasionally we stopped arguing long enough to work things out and write them down. There were a number of original contributions, notably the calculations for the $s$ and $r$ processes. The rest consisted of an extensive updating of previous work. But one should not forget the introduction of a lettering notation for the various nuclear processes: $\alpha, e, s, r, p$, and $x$, which may have done more for the development of the subject than almost anything else!

\section{INTRODUCTION}

As of 1957 enough evidence had been assembled for a review of what was known about nucleosynthesis in stars. The data (which would now be called fragmentary, though it then appeared to be spectacular) allowed Burbridge, Burbridge, Fowler, and Hoyle, $\mathrm{B}^{2} \mathrm{FH},{ }^{1}$ to combine progress in stellar and solar system abundances with laboratory nuclear physics data and stellar evolution calculations to show how stars can produce elements and their isotopes from helium to uranium. Their paper provided a basis for nuclear astrophysics for the decades that followed. How well did they do? In this review we will first outline the basic processes that they suggested to be the sources of elements heavier than hydrogen and evaluate progress in confirming and extending their suggestion.

\footnotetext{
${ }^{1}$ This was probably the first astronomical paper to be referred to by the initials of its authors.
}

\section{A. The cosmological foundations of $\mathrm{B}^{2} \mathrm{FH}$}

In 1957 there were two basic cosmological models, though neither was sufficiently developed to deserve the term "theory." These were, of course, Big Bang and steady state. The Big Bang obviously explained the expansion of the Universe but failed to predict the origin of the elements beyond the lightest species. Steady state provided for an understanding of the discrepancy between the expansion age and the apparent ages of the globular clusters - a problem that is still with us-but provided no physical basis for the continuous creation of matter, the expansion, or the collection of diffusely created matter into galaxies.

One of the strengths of the $\mathrm{B}^{2} \mathrm{FH}$ paper was its independence of the cosmological models then under discussion. The paper explained just how much stars could contribute to the synthesis of nuclei heavier than hydrogen. Since they did not have the answers for $\mathrm{D}, \mathrm{Li}, \mathrm{Be}$, and B they relegated them to the " $x$ process." Almost all other elements and isotopes could be produced in the stellar environment by one of their eight processes (with their " $x$ process" included as number eight).

\section{B. The astronomical background in 1957}

Starting in the late 1940s and early 1950s astronomers were assembling data to show that all stars did not have the same chemical composition. For 80 years it had been known that some red giants, referred to as carbon stars, showed molecular bands of carbon molecules in their spectra while the vast majority of cool stars showed oxide bands. Due to blending of absorption lines and molecular bands a quantiative comparison of carbon and oxygen red giants was not possible. More recently the subdwarfs had been found to be metal-poor by factors more than 10 (Chamberlain and Aller, 1951), and the heavy element stars such as the Ba II stars and S stars were known to show abundance excesses of certain species by factors near 10 (Burbidge and Burbidge 1957). Of great importance was the discovery of Tc in S stars (Merrill, 1952) since the longest lived isotope of Tc has a half-life of $4 \times 10^{6}$ years and Cameron (1955) had shown that ${ }^{99} \mathrm{Tc}$, with a half-life of only $3 \times 10^{5}$ years was the most easily produced isotope. This proved beyond any doubt that nucleosynthesis took place within stars and that the products could reach the stellar surface, with the help of mass loss and mixing.

\section{The eight processes}

In order to produce (almost) all known nuclear species in stars $\mathrm{B}^{2} \mathrm{FH}$ suggested eight separate processes.

\section{Hydrogen burning}

Following the fundamental papers by Bethe and Critchfield (1938) and Bethe (1939), B ${ }^{2} \mathrm{FH}$ described the laboratory experiments and the derived reaction rates of the various proton captures of the $p p$ chain and the CNO cycle. The details of the rates of the individual reactions in the $p p$ chain determine the energy spectrum 
of the resulting neutrinos, which is crucial in attempts to understand the solar neutrino problem. In addition they discussed the $p$ capture by the neon isotopes to produce ${ }^{23} \mathrm{Na}$ (also mentioned in Bethe's 1939 paper). In Secs. IV and VII, Parker and Champagne bring us up to date on the laboratory rates of hydrogen burning reactions from the $p p$ reaction through the $\mathrm{CNO}$ cycle, the $\mathrm{NeNa}$ cycle and the $\mathrm{MgAl}$ cycle. In addition the hot $\mathrm{CNO}$ cycle plays an important role in nova explosions, while the $r p$ process discussed by Boyd in Sec. XIII may be responsible for the production of certain $p$-rich isotopes.

\section{Helium burning}

By the time of $\mathrm{B}^{2} \mathrm{FH}$ helium burning to produce ${ }^{12} \mathrm{C}$ had been suggested by Opik (1951) and its rate estimated by Salpeter (1952). In Sec. VI. G. Hale describes the present state of the experiments that located and measured the width of the vital $7.65 \mathrm{MeV}$ level in ${ }^{12} \mathrm{C}$ (predicted by Hoyle) and the complicated combination of experiment and theory that is necessary to estimate the rate of the ${ }^{12} \mathrm{C}(\alpha, \gamma){ }^{16} \mathrm{O}$ reaction. The rate of the ${ }^{12} \mathrm{C}(\alpha, \gamma){ }^{16} \mathrm{O}$ reaction relative to the $3-\alpha$ process determines the carbon/oxygen ratio in massive stars, and this is crucial for the later evolution of such a star and its resulting nucleosynthesis. Unfortunately, 40 years after $\mathrm{B}^{2} \mathrm{FH}$, the rate of the ${ }^{12} \mathrm{C}(\alpha, \gamma){ }^{16} \mathrm{O}$ reaction is still not well determined. Fortunately the material that is returned to the interstellar medium by stars that are less massive than $11 M_{\odot}$ and evolve into white drawfs has been enriched by matter that has experienced only partial helium burning, so the uncertainty in the ratio of reaction rates plays a minor role.

\section{The $\alpha$ process}

$\mathrm{B}^{2} \mathrm{FH}$ suggested that further $\alpha$ captures could extend nucleosynthesis beyond ${ }^{16} \mathrm{O}$ to ${ }^{20} \mathrm{Ne},{ }^{24} \mathrm{Mg}$, etc., up to the very stable, doubly magic nucleus, ${ }^{40} \mathrm{Ca}$. However, after experiments showed that the ${ }^{16} \mathrm{O}(\alpha, \gamma){ }^{20} \mathrm{Ne}$ rate is very slow in stellar interiors, it became evident that carbon and oxygen burning are responsible for the origin of species from $\mathrm{Ne}$ to $\mathrm{S}$, with the nuclei consisting of integral numbers of $\alpha$ particles dominating the abundance curve in this region.

\section{The $e$ process}

At very high temperatures, about 4 or $5 \times 10^{9} \mathrm{~K}$, so many reactions take place that the nuclei settle down to statistical equilibrium dominated by the most tightly bound nuclei around ${ }^{56} \mathrm{Fe}$. Such conditions are reached only in supernovae. The observation of $\gamma$ rays from SN1987A due to the deexcitation of ${ }^{56} \mathrm{Fe}$, resulting from the $\beta$ decay of ${ }^{56} \mathrm{Ni}$, has demonstrated the importance of the production of iron-peak species in supernova explosions. Modern calculations of the iron-peak abundances are discussed by Meyer in Sec. XV.

\section{The $s$ process}

Beyond the iron-peak, utilizing neutrons produced by reactions such as ${ }^{13} \mathrm{C}(\alpha, n){ }^{16} \mathrm{O}$ and ${ }^{22} \mathrm{Ne}(\alpha, n){ }^{25} \mathrm{Mg}$, nuclei can be produced along or adjacent to the valley of stability via a process in which sequential neutron captures take place on a time scale that is slow compared to the beta-decay lifetime of these nuclei. This process can continue all the way up to lead and bismuth; beyond bismuth the resulting nuclei alpha decay back to $\mathrm{Pb}$ and $\mathrm{Tl}$ isotopes. In Secs. X and XI Käppeler and Smith bring us up to date on laboratory measurements, stellar models (also discussed in Sec. III by Iben), and abundance studies of the $s$-process elements.

\section{The $r$ process}

$\mathrm{B}^{2} \mathrm{FH}$ showed that, in addition to the $s$ process, there must be another neutron capture process in which the sequential neutron captures take place on a time scale which is much more rapid than the beta decay of the resulting nuclei. This process produces the much more neutron-rich progenitors that are required to account for the second set of abundance peaks that are observed about 10 mass units above the $s$-process abundance peaks corresponding to the neutron magic numbers, $N=50$ and 82 . Historically, the $r$ process has been associated with SN explosions, and in the past decade interest has focused more specifically on the neutrino-heated atmosphere surrounding the newly formed neutron star as the $r$-process site. In Sec. XII Hoffman and Timmes review both the physics and astrophysical scenario of rapid neutron capture during the explosion of massive supernovae.

\section{The $p$ process}

There are some relatively rare proton-rich nuclei such as ${ }^{92}$ Mo that are impossible to produce by $n$ capture alone. They may be produced by $p$ capture at high enough temperatures to overcome the huge coulomb barrier or by $(\gamma, n)$ reactions during supernova explosions. Recent work on $(p, \gamma)$ and $(\gamma, n)$ reactions including the $r p$ process are reviewed by Boyd in Sec. XIV.

\section{The $x$ process}

None of the above processes can produce $\mathrm{D}, \mathrm{Li}, \mathrm{Be}$, or $\mathrm{B}$, all of which are burned by $p$ capture at low temperatures in stars but hardly ever (except for ${ }^{7} \mathrm{Li}$ ) produced in stars. $\mathrm{B}^{2} \mathrm{FH}$ did not know how they were produced so they ascribed their synthesis to the $x$ process. Modern cosmological models of big bang nucleosynthesis are tuned to produce $\mathrm{D},{ }^{3} \mathrm{He},{ }^{4} \mathrm{He}$, and some ${ }^{7} \mathrm{Li}$ to fit observations of these species in very metal-poor stars and other astrophysical sources. The observations and theories of production of ${ }^{7} \mathrm{Li}, \mathrm{Be}$, and $\mathrm{B}$ in stars are reviewed by Boesgaard in Sec. V. For a brief review of the production of $\mathrm{D},{ }^{3} \mathrm{He},{ }^{4} \mathrm{He}$, and ${ }^{7} \mathrm{Li}$ in the early universe with further references see Olive and Schramm (1996). 


\section{Neutrino astrophysics}

\section{The solar neutrino problem}

In assembling this review we have decided to omit the solar neutrino problem because it has been developing so rapidly and hence is reviewed so frequently that, within the limited space available, we could not add substantially to the material already available. For the fundamentals of neutrino astrophysics see Bahcall (1989) and for a recent and thorough review see Haxton (1995). Some historical and summarizing remarks regarding the solar neutrino problem follow. For a much more detailed and very interesting history of the solar neutrino problem see the Appendix of Bahcall (1989).

The important place of neutrinos in present day astrophysics could hardly have been anticipated by $\mathrm{B}^{2} \mathrm{FH}$, which predated neutrino physics as an experimental science and the modern understanding of neutrino interactions. The possibility of detecting solar neutrinos had been suggested at the time of $\mathrm{B}^{2} \mathrm{FH}$, but not in the usual literature. Pontecorvo (1946) and Alvarez (1949) independently called attention to the possibility of the detection of neutrinos. The basic papers calling attention to the possibility of detecting solar neutrinos with a ${ }^{37} \mathrm{Cl}$ detector were published in 1964 (Davis, 1964; Bahcall, 1964). In 1968 Davis, Harmer, and Hoffman reported the first results of their $\mathrm{Cl}$ experiment in the Homestake Mine in South Dakota. Over the next 20 years or so continued observations by Davis and his colleagues and improved solar models by Bahcall and his colleagues (as well as others) demonstrated a discrepancy of a factor of 3 between the predicted number of ${ }^{8} \mathrm{~B}$ neutrinos and the detection rate for neutrinos with energy above 0.814 $\mathrm{MeV}$. No likely solar models have been able to explain the discrepancy. However, the suggestion by Mikheyev and Smirnov (1985) of matter-enhanced neutrino oscillations, following Wolfenstein's (1978) discussion of neutrino effective masses in matter, provides a very natural explanation for the observations, assuming massive neutrinos and flavor mixing. This possibility is commonly known as the MSW mechanism.

Neutrino astrophysics was given a great boost in the last decade by the results from three new solar neutrino experiments, one using a water Cerenkov detector to measure ${ }^{8} \mathrm{~B}$ neutrinos (Kamiokanda II/III) and two others using radiochemical ${ }^{71} \mathrm{Ga}$ detectors (SAGE and GALLEX). The threshold of the ${ }^{71} \mathrm{Ga}\left(\nu_{e}, e\right){ }^{71} \mathrm{Ge}$ reaction is sufficiently low, $0.233 \mathrm{MeV}$, that it is sensitive to the flux of $p p$ neutrinos produced in the first step of the $p p$ chain. As a steady-state sun must burn enough protons to account for the measured solar luminosity, there is a minimum value for neutrino capture in this detector of about 77 SNU (solar neutrino units), provided neutrinos behave as in the standard electroweak model. The measured value appears to be just consistent with this minimum value. However, the combined results of these three experiments are not fit very well by any combination of $p p,{ }^{7} \mathrm{Be}$, and ${ }^{8} \mathrm{~B}$ neutrinos, and imply an almost total absence of neutrinos from the ${ }^{7} \mathrm{Be}$ $\left(e, \nu_{e}\right){ }^{7} \mathrm{Li}$ reaction. This is very difficult to achieve in solar models given that the Kamioka II/III detector sees a substantial number of the associated ${ }^{8} \mathrm{~B}$ neutrinos (about half the standard solar model value).

This discrepancy now constitutes a major problem whose solution in terms of solar modeling, nuclear reaction rates, or neutrino physics has not yet become evident. The most recent solar models by Bahcall, Pinsonnealt, and Wasserburg (1995) which include diffusion of both helium and heavy elements actually predict slightly larger fluxes than do their models without diffusion. It appears that either new particle physics, such as the MSW mechanism (Haxton, 1995, Fig. 16), or rather desperate changes in the astrophysics, such as the mixing mechanism suggested by Cummings and Haxton (1996), will be needed.

\section{Other aspects of neutrino astrophysics}

Neutrinos play additional roles in stellar evolution and nucleosynthesis. At temperatures above about $10^{9}$ $\mathrm{K}$ the energy of the photons is sufficiently great to achieve an equilibrium of electrons and photons described by $\gamma+\gamma \leftrightarrow e^{+}+e^{-}$. There remains a very small probability (about once in $10^{19}$ interactions) that $e^{+}+e^{-} \rightarrow \nu+\bar{\nu}$ which is irreversible, so the neutrinos escape from the star. The consequent loss of energy can exceed the photon loss from the stellar surface, and thus greatly accelerate the evolution of massive stars as their temperatures rise above $10^{9} \mathrm{~K}$. In addition, at the very high densities achieved in stars that are evolving into white dwarfs, the gas is both hot and degenerate. Electromagnetic waves, when quantized into what are called plasmons, can decay into a $\nu+\bar{\nu}$ pair. These neutrinos carry off energy and accelerate the cooling and evolution of the central regions of those stars. For a full discussion of these processes see Bahcall (1989).

As the core of a massive star collapses to form a neutron star, the flux of neutrinos in the overlying shells of heavy elements becomes so great that, despite the small cross section, substantial nuclear transmutation is induced. Neutrinos, especially the higher energy $\mu$ - and $\tau$-neutrinos, can excite heavy elements and even helium to particle unbound levels. The evaporation of a single neutron or proton, and the back reaction of these nucleons on other species present, can significantly alter the outcome of traditional nucleosynthesis calculations. For example, the large abundance of ${ }^{20} \mathrm{Ne}$ in the neonburning shell may be the source in nature of ${ }^{19} \mathrm{~F}$, a relatively rare element, which is bypassed by most proton and $\alpha$-particle induced reactions Woosley et al. (1990), and Timmes, Woosley, and Weaver (1995). See also Jorissen et al. (1993) and Forestini et al. (1992), who discuss ${ }^{19} \mathrm{~F}$ production by $n$ capture). This is now called the "neutrino-", or $\nu$-process, and should be added to the eight processes suggested by $\mathrm{B}^{2} \mathrm{FH}$. 


\section{E. Related reviews}

There have been a number of reviews of nucleosynthesis in stars during the past 40 years. Trimble (1975) reviewed the situation very completely at about the halfway point between the publication of $\mathrm{B}^{2} \mathrm{FH}$ and the present (see also Trimble, 1991, 1995). More recently Wheeler, Sneden, and Truran (1989) reviewed stellar abundances and their nucleosynthetic origins. The monograph by Arnett (1996) includes a substantial amount of material on stellar abundances and their origins. We have not covered galactic nucleosynthesis but point to the excellent monograph by Pagel (1997). These are only samples of a substantial number of reviews that have been published within the past 20 years.

\section{STELLAR EVOLUTION}

\section{A. Historical preliminary}

Tremendous progress has been made in the field of stellar evolution since publication of the $\mathrm{B}^{2} \mathrm{FH}$ article in 1957. Much of what the authors anticipated in the way of element and isotope synthesis in stars has been borne out by model calculations, certainly in conceptual terms, if not in precise detail. $\mathrm{B}^{2} \mathrm{FH}$ noted, however, that "the whole problem of stellar evolution beyond the red giant stage is beset on the theoretical side by problems which are very difficult to handle with the present computational techniques." It is now apparent that much of the progress in modeling that has occurred over the past forty years is a consequence of the remarkable increase in the speed and memory of computers and of the introduction by Henyey, Forbes, and Gould (1964) of an implicit relaxation method for solving the equations of stellar structure.

On the other hand, all of the improvement in the world in computational facilities and methods of solution would have done little to advance the subject had the input physics remained inadequate. The absence of models beyond the shell hydrogen-burning red giant stage about which $\mathrm{B}^{2} \mathrm{FH}$ express concern was partly a consequence of an incomplete quantitative description of the physical conditions encountered by stars evolving beyond hydrogen-burning stages.

Although it was established by 1957 that the second excited state in the ${ }^{12} \mathrm{C}$ nucleus plays a crucial role in the burning of ${ }^{4} \mathrm{He}$ by the $3 \alpha$ process, the appropriate value for the gamma width of this state was uncertain by at least an order of magnitude. Strong neutrino losses in the stellar interior during advanced stages of evolution of intermediate mass stars play an absolutely crucial role in determining the relationship between the initial mass of the star and the mass of the white dwarf into which it evolves, but the current-current theory of weak interactions that predicts these losses was not invented until 1958 (Feynman and Gell-Mann, 1958) and the corresponding energy loss rates were not fully worked out until the late 60's (Beaudet, Petrosian, and Salpeter, 1967; Festa and Ruderman, 1969). The possibility of neutral-current contributions to neutrino losses entered physics a decade later with the unified electroweak theory (Weinberg, 1967, Salam, 1968), but calculation of the (15-30\%) contributions of these currents did not begin until several years later (Dicus, 1972, Dicus et al., 1976). Exploration of the full range of parameter space relevant for stellar physics is just now being completed (Itoh et al., 1996 and references therein).

Rosseland mean opacities in the continuum approximation for mixtures of hydrogen and helium were available (Keller and Meyerott, 1955), but the inclusion of line transitions in a systematic way did not occur until a decade later (Cox, 1965; Cox et al., 1965), and opacities for mixtures appropriate for more advanced stages and with line transitions included did not become available until even later (e.g., Cox and Stewart, 1970a, 1970b). At about the same time, accurate electron conductivities became available in the nonrelativistic regime (Hubbard and Lampe, 1969) and in the relativistic regime (Canuto, 1970). More recently, advances in computer capabilities, the increasing volume of requisite atomic data, and the sophistication of the atomic and statistical physics employed have led to major improvements in the determination of opacities in stellar envelopes (Iglesias, Rogers, and Wilson, 1990; Seaton et al., 1994), and opacities for a wide range of conditions in the interior have become available (Rogers and Iglesias, 1992; Iglesias and Rogers, 1996, and references therein). Improvements have, of course, also been made in the equation of state.

Finally, the huge increase in the wavelength range accessible to observers that has been brought about by technological advances in detectors and telescopes and by the move of telescopes and detectors into space has vastly increased the contact between theoretical model predictions and the real world, permitting a test of those phases of evolution in which most of the emitted light is outside of the optical range.

In the following, Sec. III.B will focus on the evolution of single stars of low and intermediate mass evolving into white dwarfs (WDs) after passing through a planetary nebula (PN) stage; Sec. III.C will focus on the evolution of massive stars evolving into neutron stars (NSs) or black holes (BHs) after a type II supernova (SNII) explosion; and Sec. III.D will focus on the evolution of close binary stars that can evolve into (a) cataclysmic variables (CVs) that experience nova outbursts, (b) a pair of WDs that can merge to produce a type Ia supernova (SNIa) or R Corona Borealis ( R CrB) star, or (c) an $\mathrm{x}$-ray binary in which the accretor has experienced a type Ib or type Ic supernova explosion (SNIb,c) to become a $\mathrm{NS}$ or $\mathrm{BH}$, and the mass donor is a low mass main-sequence star or subgiant (LMXBs) or a high mass star of spectral type OB (HMXBs).

\section{B. Evolution of single stars that become white dwarfs}

\section{Overview}

Some of what has been learned by modeling since the time of $\mathrm{B}^{2} \mathrm{FH}$ is summarized in the H-R diagram of Fig. 


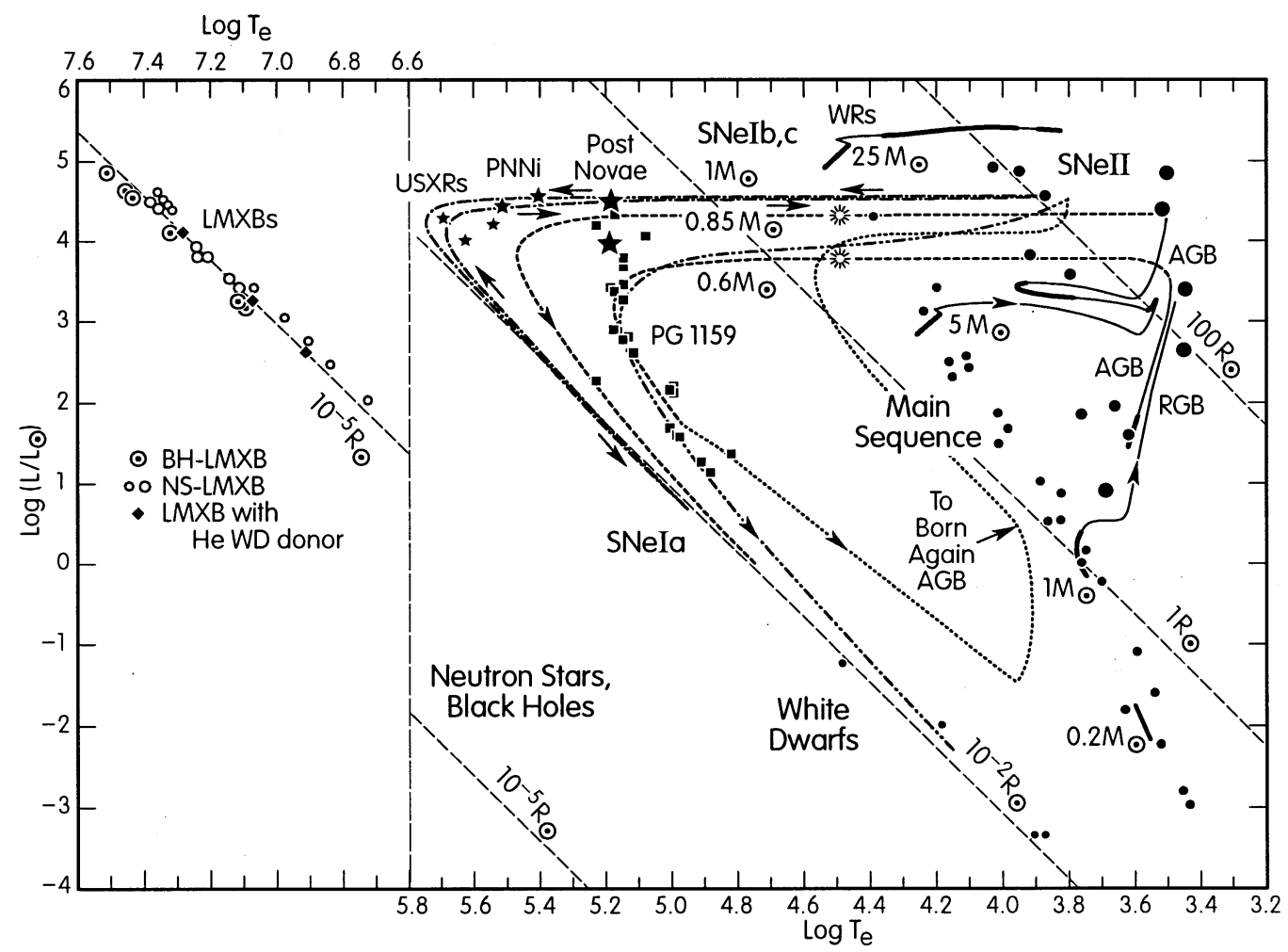

FIG. 1. Theory and observation compared in the theoretical H-R diagram. For guidance, lines are shown along which stellar radius is constant (dashed lines of negative slope). Solid curves in the right-hand portion of the figure are evolutionary tracks for single stars of mass $25,5,1$, and $0.2 M_{\odot}$. Heavy portions of the tracks denote phases of core nuclear burning. Dashed curves labeled $0.6 M_{\odot}$ and $0.85 M_{\odot}$ are evolutionary tracks following the superwind state on the AGB during which model stars of initial mass $1 M_{\odot}$ and $5 M_{\odot}$, respectively, lose most of their hydrogen-rich envelopes and evolve into white dwarfs; "sunburst" symbols along these tracks show where hard photons from the contracting central star are emitted sufficiently frequently to excite the ejected matter into fluorescence as a planetary nebula. Solid circles are observed white dwarfs (smallest), core hydrogen-burning main-sequence stars (next largest), core helium-burning stars (second to largest), and red giants or red supergiants (largest) burning hydrogen and/or helium in a shell (most data from Allen, 1973). Shown also is the track of a model of mass $0.6 M_{\odot}$ which experiences a final helium shell flash after having become a white dwarf and evolves into a "born again" AGB star. The dash-dotted track describes the path of a model white dwarf of mass $1 M_{\odot}$ accreting matter from a hydrogen-rich companion (Iben, 1982); after accreting a critical mass, the model experiences a nova explosion and evolves to high luminosity before returning to the white dwarf state. The solid star symbols describe ultra-soft x-ray binaries (USXRs, smallest), central stars of post classical novae (Post Novae, next largest), and central stars of planetary nebulae (PNNi, largest) (see Iben and Tutukov, 1996a for references). The maximum luminosity of low mass x-ray binaries for which distance estimates are available are shown in the left-hand panel along a line of constant radius comparable to the radius of a $1.4 M_{\odot}$ neutron star or a $10 M_{\odot}$ black hole $($ data from van Paradijs, 1995; van Paradijs and White, 1995; and Tanaka and Lewin, 1996, as used by Iben and Tutukov, 1977).

1. In the rightmost portion of this figure $\left(\log T_{\mathrm{e}} \approx 4.7\right)$, evolutionary tracks of model single stars (of initial composition close to that at the Sun's surface and of initial masses $0.2,1,5$, and $25 M_{\odot}$ ) are shown together with the positions of a selection of bright and nearby optically visible real stars. The tracks are defined by models that are solutions of the equations of stellar structure, including nuclear transformations at rates based either on laboratory cross-section measurements or on wellestablished weak interaction theory. Heavy portions along the tracks indicate where evolution proceeds on a core nuclear-burning time scale. The real stars are represented by filled circles of four different sizes (1 $=$ smallest, $4=$ largest, etc.); most of them are from Allen (1973). Figure 1 is very busy and the reader might find it useful to examine simultaneously Figs. 1 and 5 in Iben (1991a) and Fig. 1 in Chiosi et al. (1992).
One of the strongest arguments for the general notion that heavy elements are made in stars is the existence of three different sequences defined by real stars that can be understood in terms of the nuclear burning evolutionary models. Apparent discrepancies between the location of real stars in a given sequence and the locus defined by connecting the heavy portions of theoretical tracks for the relevant nuclear burning stage can in most cases be understood as consequences of uncertainties in observational estimates or of inapproriate choices of the parameters (such as initial composition and the efficiency of convection) chosen for the theoretical models.

Size 2 filled circles in Fig. 1 define a "main sequence" that is clearly coincident with the band defined by model stars that are burning hydrogen in their cores (the first heavy portion of the tracks). The main-sequence lifetime is inversely proportional to roughly the 2.25 power 
of the stellar mass, decreasing from about a Hubble time $\left(\sim 10^{10}\right.$ years $)$ for a star of mass $\sim 1 M_{\odot}$ to $\sim 7 \times 10^{6}$ years for a star of mass $\sim 25 M_{\odot}$. A star less massive than $0.8-1.0 M_{\odot}$ (depending on the composition) does not leave the main sequence in less than a Hubble time; this accounts for the shortness of the track displayed in Fig. 1 for the $0.2 M_{\odot}$ model.

Size 3 filled circles in Fig. 1 define a sequence that is coincident with the band defined by model stars burning helium in their cores and hydrogen in a shell (the second heavy portion along the $1 M_{\odot}$ and $25 M_{\odot}$ tracks and the third heavy portion of the $5 M_{\odot}$ track). Hydrogen burning provides most of the luminosity, but helium burning sets the time scale, which varies from about $25 \%$ of the main-sequence lifetime for intermediate mass stars to about $10 \%$ for massive stars. Models less massive than $\sim 2.3 M_{\odot}$ have roughly the same helium core mass during this stage and therefore have nearly the same luminosity $\left(\sim 50 L_{\odot}\right)$ and lifetime $\left(\sim 10^{8}\right.$ years).

Size 4 filled circles define a third, red giant, red supergiant sequence that can be understood in terms of the following: (a) models of initial mass $\approx 2.3 M_{\odot}$ with an inert electron-degenerate helium core and a hydrogenburning shell (e.g., that portion of the $1 M_{\odot}$ evolutionary track labeled RGB, the red giant branch); (b) models of mass in the range $2.3-20 M_{\odot}$ during a first phase of core helium burning and shell hydrogen burning (e.g., the second heavy portion of the $5 M_{\odot}$ evolutionary track preceding the main core helium-burning phase); and (c) models that have a neutrino-cooled electron-degenerate core composed of carbon and oxygen (CO core) (if initial mass is in the range $1-9 M_{\odot}$ ) or of oxygen and neon (ONe core) (if initial mass is in the range 9-11 $M_{\odot}$ ) and that are burning alternately hydrogen and then helium in shells. The case (c) models are known as "asymptotic giant branch (=AGB)" stars because the track of a model of low mass in this phase (e.g., the portion of the $1 M_{\odot}$ track in Fig. 1 labeled AGB) is asymptotic to the track during the RGB phase.

Another, clearly discernable sequence defined by optically selected stars is that of white dwarfs (size 1 filled circles at low luminosity in Fig. 1). The formation rate of white dwarfs $\left(\sim 0.5-1 \mathrm{yr}^{-1}\right)$ that is estimated by comparing the observed number-luminosity distribution of nearby white dwarfs with models of cooling white dwarfs is consistent with the formation rate of stars in the mass range $1-11 M_{\odot}$ determined by comparing the number-luminosity distribution of main-sequence stars with the model main-sequence lifetimes. This consistency supports the view (based on more detailed comparisons between theory and observation) that all stars that develop an electron-degenerate core along the giant branch shed their hydrogen-rich envelope during the AGB phase to become, first, the central star of a planetary nebula (which is the envelope that has been shed and which is excited into fluorescence by hard photons from the central star) and then a white dwarf.

The observation by Deutsch (1956) that demonstrated mass loss at a high rate from the M supergiant $\alpha$ Herculis and the speculation by Hoyle (1956) that, in the words of $\mathrm{B}^{2} \mathrm{FH}$, "mass loss may have a more important effect on the evolution of giants and supergiants of very low surface gravity than have nuclear processes" was slow to be incorporated into theoretical models of giants and supergiants. The first explicit calculation was that of Paczyński (1971a) and the second was that of Härm and Schwarzschild (1975). Both calculations consisted of removing mass from the surface of a model AGB star at a rate large compared to the rate at which matter is being processed in the interior by nuclear reactions, and both calculations showed that, once the mass of the hydrogen-rich envelope is reduced below a very small critical value, the model rapidly evolves to the blue in the H-R diagram. The dashed tracks in Fig. 1 labled 0.6 $M_{\odot}$ and $0.85 M_{\odot}$ are the consequence of similar calculations. The "sunburst" (rayed open circles) along these tracks indicate where photons emitted from the surface of the contracting stellar remnant are hard enough to ionize hydrogen atoms in the ejected material and to excite this material into fluorescence. The resulting object is known as a planetary nebula. The location of two relatively massive planetary nebula nuclei $(\mathrm{PNNi})$ are shown by the star symbols of intermediate size. A typical PNN has a mass $\sim 0.55-0.6 M_{\odot}$ and burns hydrogen in a thin shell near its surface for a few times $10^{4}$ years (Schönberner, 1981). This is consistent with the fact that the number-mass distribution of white dwarfs in the solar vicinity peaks somewhere in the $\sim 0.55-0.65 M_{\odot}$ range (Liebert and Bergeron, 1995).

Observation-based estimates of mass-loss rates from Mira variables and other acoustically pulsating AGB stars have shown that, once the pulsation period exceeds $\sim 400$ days, the rate of mass loss becomes of the order of $10^{-5}-10^{-4} M_{\odot} \mathrm{yr}^{-1}$, or typically $10^{2}-10^{3}$ times larger than the rate at which nuclear burning processes matter in the interior. This circumstance shows that a theoretical mapping between initial mass and final white dwarf mass is possible - the white dwarf mass is essentially the mass of the electron-degenerate core of a model when it first becomes a double-shell-burning AGB star augmented by only several hundredths of a solar mass (processed by nuclear burning before the hydrogen-rich envelope is removed by the stellar wind).

The AGB phase of mass loss at a high rate (sometimes called the "superwind" phase [Renzini, 1981] and sometimes called a planetary nebula ejection event) is due to a combination of effects (see, e.g., Bowen, 1988; Bowen and Willson, 1991): (1) pulsation (hard to calculate because the envelope is convective) leads to shocks; (2) shock heating inflates the atmosphere; (3) dust grains are formed and grow in low temperature regions of the atmosphere during the pulsation cycle; and (4) radiation pressure drives the grains to higher-than-escape velocity, and the grains drag the gas along.

\section{Nucleosynthesis and dredge-up prior to the AGB phase}

One of the recurrent themes in $\mathrm{B}^{2} \mathrm{FH}$ is that the exotic abundances found at the surfaces of several groups of evolved stars (e.g., R CrB stars, Wolf-Rayet stars, 
carbon stars) might be the consequences of nucleosynthesis, mixing, and mass-loss processes taking place in single stars and that the different abundance patterns might be correlated with the evolutionary stage in which a star finds itself. Subsequent theoretical calculations have shown this to be true in several instances, but, as recognized by $\mathrm{B}^{2} \mathrm{FH}$, some exotic surface abundances can be more easily understood in terms of binary star evolution during which mass is lost and transferred, sometimes to the extent of a complete merger.

There are four major dredge-up episodes which single stars can experience. During each episode, turbulent convection carries to the surface products of nucleosynthesis in the interior. The first episode occurs after hydrogen is exhausted over a substantial fraction of the interior (about $10 \%$ of the mass of the star if the mass of the star is less than $\sim 2.3 M_{\odot}$ [Schönberg and Chandrasekhar, 1942] or about $0.1 M_{\odot}\left(M / M_{\odot}\right)^{1.4}$ for larger masses $M$ [Iben and Tutukov, 1984]), and the hydrogenrich envelope expands in response to the contraction and heating of the helium core. The decreasing temperatures and densities in the expanding envelope lead to increasing opacities and a steepening of the temperature profile until turbulent convection is forced to carry the outward flux of energy emerging from the hydrogenburning shell (Hoyle and Schwarzschild, 1955). As the base of the convective envelope moves inward in mass, it sweeps first into regions where fragile elements such as lithium have been destroyed by reactions with protons, next into a region where primordial ${ }^{12} \mathrm{C}$ has been converted into ${ }^{13} \mathrm{C}$ by the reactions ${ }^{12} \mathrm{C}(p, \gamma){ }^{13} \mathrm{~N}\left(e^{+}, \nu\right){ }^{13} \mathrm{C}$, then into a region where most of the primordial ${ }^{12} \mathrm{C}$ has been converted into ${ }^{14} \mathrm{~N}$, and, in the most massive stars, into a region where ${ }^{16} \mathrm{O}$ has been converted into ${ }^{14} \mathrm{~N}$. Thus, the surface abundance of lithium drops first, followed by a decrease in the ratio ${ }^{12} \mathrm{C} /{ }^{13} \mathrm{C}$, a drop in the surface abundance of ${ }^{12} \mathrm{C}$, and an increase in the abundance of ${ }^{14} \mathrm{~N}$, and finally, in some stars, by a decrease in the surface abundance of ${ }^{16} \mathrm{O}$ and a second increase in the abundance of ${ }^{14} \mathrm{~N}$.

The predictions regarding changes in the surface abundances of CNO elements (Iben, 1964) are in reasonable agreement with the observations for field giants (Lambert and Ries, 1981). The predictions regarding changes in the surface lithium abundance in metal-rich stars (Iben, 1965, 1967b) are in reasonable agreement with the observations for red giants more massive than $\sim 1.3 M_{\odot}$ (Lambert, Dominy, and Sivertsen, 1980; Pilachowski, 1986), but they underestimate the depletion of lithium by as much as two orders of magnitude for lower mass giants (Luck and Lambert, 1982), suggesting that other mixing mechanisms involving convective overshoot or differential rotation are much more important than the standard first dredge-up mechanism. Excellent modern discussions of expected versus observed isotopic abundances of $\mathrm{CNO}$ elements are given by Dearborn (1992), El Eid (1994), and Prantzos et al. (1996).

In stars less massive than $\sim 2.3 M_{\odot}$, the helium core becomes electron-degenerate before helium is ignited. The core is kept roughly isothermal by electron conduc- tion, but plasma neutrino losses ensure that the maximum temperature in the star is not at the center. As the core grows in mass, gravitational potential energy is converted into heat and eventually, when the mass of the core reaches $\sim 0.45-0.5 M_{\odot}$, helium is ignited and burns in a series of flashes that work their way to the center, lifting the degeneracy (Mengel and Sweigart, 1981). The star becomes a "clump" star (the heavy line segment along the $1 M_{\odot}$ track at $L \sim 50 L_{\odot}$ in Fig. 1) and converts helium into carbon under non-electron-degenerate conditions. In more massive stars, helium ignites in the helium core before electron degeneracy sets in.

Once helium is exhausted in model stars less massive than $\sim 9 M_{\odot}$ (for solarlike initial composition), the $\mathrm{CO}$ core becomes electron degenerate and, due to neutrino losses, cools to the extent that carbon does not ignite. Helium burning in a shell provides most of the energy escaping from the surface. In models less massive than $\sim 5 M_{\odot}$, when the helium-burning shell comes close enough in mass to the hydrogen-helium discontinuity, hydrogen is reignited and the model enters a phase of alternating hydrogen and helium burning. In models of mass in the range 5-9 $M_{\odot}$, once the mass of the $\mathrm{CO}$ core exceeds $\sim 0.56-0.61 M_{\odot}$, the hydrogen-exhausted core behaves like a red giant with a helium core: the electron-degenerate $\mathrm{CO}$ core contracts and heats, while the helium rich layer above it expands and cools. The base of the convective envelope, which is initially above the hydrogen-helium discontinuity, moves inward in mass, extending eventually into the helium layer above the $\mathrm{CO}$ core. Fresh ${ }^{4} \mathrm{He}$ and ${ }^{14} \mathrm{~N}$ (into which most of the primordial $\mathrm{CNO}$ elements have been converted during hydrogen burning) are dredged into the convective envelope and appear enhanced at the surface (e.g., Iben, 1972; Becker and Iben, 1980).

This "second dredge-up" episode occurs also in model stars of mass in the range $9-11 M_{\odot}$, but the phenomenon is much more complex since the CO core is only partially electron degenerate and both carbon-burning and helium-burning shell flashes occur; the flux of energy that reaches the base of the convective envelope and forces this base to move inward in mass can have its origin in carbon burning, helium burning, the release of gravothermal energy, or in some combination of these sources. In a $9 M_{\odot}$ model of solar initial composition, the dredge-up occurs in conjuntion with the first carbonshell flash and gravothermal energy is responsible for dredge-up (García-Berro et al., 1997). In a $10 M_{\odot}$ model, dredge-up occurs near the end of the carbon-burning phase, and both helium burning and carbon burning contribute to the dredge-up (Ritossa et al., 1996). In a $10.5 M_{\odot}$ model, dredge-up occurs also near the end of the carbon-burning phase, and all three energy sources play a role in the dredge-up process (Iben, Ritossa, and García-Berro, 1997).

\section{Nucleosynthesis and dredge-up during the AGB phase}

Approximately $97 \%$ of all stars that can leave the main sequence in less than a Hubble time become AGB 
stars and experience thermal pulses due to helium shell flashes (Schwarzschild and Härm, 1965; Weigert, 1966) which periodically interrupt the normal, quiescent hydrogen-burning phase. Although their lifetime is only $\sim 10^{5}-10^{6}$ years (depending on the initial mass), thermally pulsing AGB (TPAGB) stars produce much of the ${ }^{12} \mathrm{C}$ and most of the $s$-process isotopes in the Universe. They are also responsible for most of the nitrogen (the ${ }^{14} \mathrm{~N}$ entering the convective envelope during the first and second dredge-up phases plus the ${ }^{14} \mathrm{~N}$ made by proton burning at the expense of the ${ }^{12} \mathrm{C}$ that enters the convective envelope following helium-shell flashes in the "third dredge-up" process described below).

The radius of the $\mathrm{CO}$ or ONe core of a TPAGB star is similar to that of a white dwarf of the same mass into which the core eventually evolves. Helium shell flashes occur for the same reason that white dwarfs accreting matter at a rate smaller than is necessary to support quiescent helium burning experience nova explosions when the mass of accreted helium reaches a critical value which is larger, the smaller the accretion rate. During the quiescent hydrogen-burning phase in AGB stars, helium is deposited into a layer above the underlying $\mathrm{CO}$ or $\mathrm{ONe}$ core at a rate which is an order of magnitude smaller than that necessary to maintain quiescent helium burning. As the helium layer grows in mass, it becomes compressed and heated until eventually helium ignites. The helium-burning rate is proportional to about the 40th power of the temperature and nuclear energy is released at a rate faster than the heat into which it is converted can diffuse out radiatively. A brief thermonuclear runaway continues until it is damped out by expansion and cooling.

The large fluxes generated during a flash create a convective zone that extends from the base of the heliumburning region almost to the hydrogen-helium discontinuity. An entropy barrier, to which radiation pressure contributes significantly, prevents the outer edge of the zone from reaching hydrogen-rich material (Iben, 1976, 1977a). Prior to a flash, the helium layer that is built up contains ${ }^{14} \mathrm{~N}$ at an abundance equal to that of primordial $\mathrm{CNO}$ elements. During the first part of a flash, this ${ }^{14} \mathrm{~N}$ is converted completely into ${ }^{22} \mathrm{Ne}$ by the reactions ${ }^{14} \mathrm{~N}$ $(\alpha, \gamma){ }^{18} \mathrm{~F}\left(\beta^{+}, \nu\right){ }^{18} \mathrm{O}(\alpha, \gamma){ }^{22} \mathrm{Ne}$. In models in which the core mass is $\left\ulcorner 0.9 M_{\odot}\right.$, temperatures at the base of the convective zone can reach over $350 \times 10^{6} \mathrm{~K}$ and the reaction ${ }^{22} \mathrm{Ne}(\alpha, n){ }^{25} \mathrm{Mg}$ can be a potent source of neutrons (Iben, 1975a, 1976, 1977a; Ritossa et al., 1996) for the production of $s$-process isotopes at abundances several hundred times larger than solar (Iben 1975b; Truran and Iben, 1977). It is interesting that $\mathrm{B}^{2} \mathrm{FH}$ speculated that the ${ }^{21} \mathrm{Ne}(\alpha, n){ }^{24} \mathrm{Mg}$ reaction might be an important neutron source in stars and did not consider the ${ }^{22} \mathrm{Ne}$ $(\alpha, n)^{25} \mathrm{Mg}$ reaction (Cameron, 1961).

In AGB models with $\mathrm{CO}$ cores less massive than $\sim 0.9 M_{\odot}$, temperatures do not become large enough at the base of the convective envelope for the conversion of more than a percent or so of ${ }^{22} \mathrm{Ne}$ into ${ }^{25} \mathrm{Mg}$ (Becker, 1981; Iben, 1983). However, it has been known since the discovery of Tc in stars by Merrill (1952) that $s$-process isotopes are made in abundance in such stars and the likely neutron source is the ${ }^{13} \mathrm{C}(\alpha, n){ }^{16} \mathrm{O}$ reaction (Cameron, 1955). The manner in which the ${ }^{13} \mathrm{C}$ source is activated may not be unique and it is not completely understood.

Schwarzschild and Härm (1967) found that, in one of thirteen pulses computed, the convective shell actually reached the hydrogen-helium discontinuity and ingested some hydrogen. Sanders (1967) pursued the consequences of this, finding that the ingested protons react with the ${ }^{12} \mathrm{C}$ present at large abundance in the convective shell to produce mostly ${ }^{13} \mathrm{C}$ which diffuses convectively inward until reaching temperatures of the order of $\sim 150 \times 10^{6} \mathrm{~K}$, whereupon $\alpha-n$ reactions provide neutrons for $s$-process nucleosynthesis. The difficulty with this scenario is that the Schwarzschild and Härm (1967) models did not include radiation pressure, and the ingestion mechanism has not been found in subsequent calculations which include radiation pressure. Another set of calculations (Iben and Renzini, 1982a, 1982b; Hollowell and Iben, 1989) shows that, after the convective shell has died away in low mass AGB stars of low metallicity, semiconvective mixing forces ${ }^{12} \mathrm{C}$ and ${ }^{1} \mathrm{H}$ to overlap at comparable number abundances in a region centered at the location of the outer edge of the convective shell at its maximum extent during the flash. Ultimately, matter in this region contracts and heats, and a small pocket of ${ }^{13} \mathrm{C}$ is formed in consequence of proton capture on ${ }^{12} \mathrm{C}$ followed by a beta decay. When the next shell flash occurs, the convective shell ingests the pocket of ${ }^{13} \mathrm{C}$ and matters proceed as outlined by Sanders (1967). This mechanism does not appear to work in AGB stars of solar metallicity (Iben, 1983).

It has recently been discovered that, even if a ${ }^{13} \mathrm{C}$ pocket is formed, it may act as a local neutron source during the quiescent hydrogen-burning phase between flashes (Straniero et al., 1995), rather than as a distributed source in a convective shell during flashes; surprisingly, the $s$-process abundance distributions produced are essentially the same in both cases (Straniero et al., 1995). Still more recently, Blöcker et al. (1997) and Herwig et al. (1997) have shown that convective overshoot beyond the base of the convective envelope during the third dredge-up phase leads to the formation of a ${ }^{13} \mathrm{C}$ pocket and this, in retrospect, could have been recognized from calculations already in the literature (e.g., Iben, 1976, Fig. 9).

The "third dredge-up" event occurs during the power down phase of a shell flash, when energy produced by helium burning leaks out of the carbon-rich region, increasing the flux of energy passing through the base of the convective envelope. This increase forces the base of the convective envelope to move inward in mass into the region which contains the products of partial helium burning. This event was first encountered in an AGB model of large $\mathrm{CO}$ core mass without invoking overshoot at the base of the convective envelope (Iben, 1975a), and later in AGB models of small core mass with the help of convective overshoot (Iben and Renzini, 1982a, 1982b; Iben, 1983). More on the history of 
this phenomenon may be found in reviews by Sackmann and Boothroyd (1991) and Iben (1991b).

Helium shell flashes and the third dredge up are responsible for the formation of carbon stars (stars in which carbon is more abundant than oxygen) and for producing overabundances of $s$-process isotopes in such stars. In addition to the enhancements which occur during the first and second dredge-up phases, a further enhancement of ${ }^{14} \mathrm{~N}$ can occur as a consequence of the burning of ${ }^{12} \mathrm{C}$ into ${ }^{14} \mathrm{~N}$ at the base of the convective envelope between flashes in massive AGB stars (e.g., Iben, 1975a; Renzini and Voli, 1981). The freshly synthesized ${ }^{14} \mathrm{~N},{ }^{12} \mathrm{C}$, and $s$-process isotopes are returned to the interstellar medium during the superwind phase that converts the AGB star into a planetary nebula. Thus, AGB stars are major contributors to the enrichment of the interstellar medium in these elements and isotopes (Iben and Truran, 1978), as well as in ${ }^{4} \mathrm{He}$ (Renzini and Voli, 1981), although the Big Bang is by far the major contributor of this latter isotope. It is worth noting that the material which is dredged up during the TPAGB phase has experienced only partial helium burning, with the abundance by mass of ${ }^{12} \mathrm{C}$ in dredged-up matter being only $\sim 0.15-0.25$. This means that the uncertainty in the cross section for the ${ }^{12} \mathrm{C}(\alpha, \gamma){ }^{16} \mathrm{O}$ reaction relative to the cross section for the triple alpha reaction does not produce a similarly serious uncertainty in the abundance of carbon in dredged-up matter.

The light isotopes ${ }^{3} \mathrm{He}$ and ${ }^{7} \mathrm{Li}$ are made in intermediate mass stars in interesting quantities (relative to the Big Bang contribution of these isotopes) and returned to the interstellar medium during the superwind phase. ${ }^{3} \mathrm{He}$ is made in the central regions of all main-sequence stars (Iben, 1967b) at an abundance by mass which, when averaged over the matter outside of active hydrogen-burning regions, is $\sim 4.6 \times 10^{-4} M_{\odot} / M_{\mathrm{MS}}$ (Iben, 1977b). This ${ }^{3} \mathrm{He}$ is preserved and returned to the interstellar medium by AGB stars with initial masses at the low end of the intermediate mass range $\left(1-2 M_{\odot}\right)$, as is confirmed observationally by the high ${ }^{3} \mathrm{He} / \mathrm{H}$ ratio in planetary nebulae (Balsar et al., 1997) which have progenitor masses less than $\sim 2 M_{\odot}$. However, the low ${ }^{12} \mathrm{C} /{ }^{13} \mathrm{C}$ ratios in small-mass red giants of old galactic clusters, red giants in globular clusters, and extremely metal-poor giants in the field (see Sec. VII) suggest that ${ }^{3} \mathrm{He}$ should be depleted in a substantial fraction of their mass. Hogan (1995) has shown that this implies that the observed ${ }^{3} \mathrm{He}$ in the Galaxy may be less than the primordial ${ }^{3} \mathrm{He}+\mathrm{D}$. In higher mass AGB stars, however, ${ }^{3} \mathrm{He}$ burns with ${ }^{4} \mathrm{He}$ at the base of the convective envelope to form ${ }^{7} \mathrm{Be}$. Cameron (1955) and Cameron and Fowler (1971) suggested that, if this ${ }^{7} \mathrm{Be}$ could be mixed outward to cooler regions where it would be destroyed by the ${ }^{7} \mathrm{Be}\left(e^{-}, \nu\right)^{7} \mathrm{Li}$ reaction rather than by the ${ }^{7} \mathrm{Be}(p, \gamma){ }^{8} \mathrm{~B}^{*}\left(2{ }^{4} \mathrm{He}\right)$ reactions, this might explain the observed superabundances of $\mathrm{Li}$ in some Galactic giants (Wallerstein and Conti, 1969; Boesgaard, 1970). Of course, the mixing must also be on a long enough time scale to prevent the rapid destruction of ${ }^{7} \mathrm{Li}$ by the ${ }^{7} \mathrm{Li}(p, \alpha){ }^{4} \mathrm{He}$ reaction. Scalo et al. (1975) demonstrated that this mechanism could work in the convective envelope of intermediate mass AGB stars. Smith and Lambert $(1989,1990)$ then showed that several of the most luminous stars in the Large Magellanic cloud (LMC) which could be identified as AGB stars by an overabundance of $\mathrm{ZrO}$ in their spectra (Wood et al., 1983) are super lithium rich. This finding has been reinforced by further observations (Plez et al., 1993; Smith et al., 1995, and references therein). Finally, Sackmann and Boothroyd (1992) have constructed evolutionary models of stars in the 3-7 $M_{\odot}$ range and find that (with appropriate choices of parameters in a time-dependent convective mixing algorithm) the observed lithium abundances and luminosities of the LMC super lithium rich stars can be reproduced by models of initial mass in the range 4-6 $M_{\odot}$.

\section{The born-again AGB phenomenon}

There are a number of observed stars and stellar systems that can be understood in terms of a final helium shell flash which a post-AGB star may experience after it has ceased to burn hydrogen. The phenomenon was predicted by Fujimoto (1977), encountered by Schönberner (1979), and exploited by Iben et al. (1983) and Iben (1984) in an attempt to explain the presence of knots of helium-rich, nitrogen-rich clumps near to, but moving with speeds of 20 to $30 \mathrm{~km} \mathrm{~s}^{-1}$ away from, the central stars of the planetary nebulae Abell 30 (Hazard et al., 1980) and Abell 78 (Jacoby and Ford, 1983).

When the flash occurs, the mass of the helium layer below the hydrogen-helium discontinuity is slightly smaller than that necessary for initiating a flash on the AGB. This is because the matter in the helium layer is partially degenerate and is heated by adiabatic contraction when the hydrogen-burning shell loses its power. The final flash is a strong one, and because the entropy barrier between hydrogen-rich matter and the helium layer is much smaller than on the AGB, the outer edge of the convective shell forced by helium burning extends into the hydrogen-rich region of the star. Protons are ingested and diffuse inward until reaching a point where reactions with ${ }^{12} \mathrm{C}$ inject so much entropy that the convective shell breaks into two parts, the lower shell being forced by fluxes from helium-burning reactions and the outer one being forced by fluxes from hydrogen-burning reactions (Iben and MacDonald, 1995).

The outer convective shell consists primarily of ${ }^{4} \mathrm{He}$ (76\% by mass) and ${ }^{12} \mathrm{C}(20 \%$ by mass), with only a few percent by mass of ${ }^{1} \mathrm{H}$, and a trace of ${ }^{16} \mathrm{O}$. The hydrogen abundance is so low because, prior to the flash, the mass of the hydrogen-rich layer is about 20 times smaller than the mass of the helium-rich layer with which it is mixed during the flash. As burning at the base of the outer convective shell (which extends to the photosphere) progresses, approximately $5 \%$ of the ${ }^{12} \mathrm{C}$ is converted into ${ }^{14} \mathrm{~N}$ by proton burning. Thus, this mode of evolution produces surface abundances that are the consequence of mixing products of partial helium burning with a small amount of hydrogen-rich matter and sub- 
jecting the mix to another bout of hydrogen burning. The track of the model that produces the quoted abundances is shown by the dotted curve in Fig. 1. The fact that the track extends to large luminosities and low surface temperatures has led to the designation "born again AGB" stars. Perhaps $10 \%$ of all post-AGB stars may experience a final helium shell flash after hydrogen burning is completed (Iben, 1984). Another 15\% may experience a final helium shell flash before hydrogen burning is finished. These too become born again AGB stars, but an entropy barrier prevents the ingestion of hydrogen into the convective shell produced by fluxes from the helium-burning region. Wind mass loss during the born again phase and thereafter may remove most if not all of the hydrogen rich surface layer, and the star may evolve into a "non-DA" white dwarf (surface abundance of hydrogen $\approx 10^{-4}$ by mass). There are now about a half dozen examples of stars that have been identified as passing through the born-again phase, the latest being Sakurai's object (Duerbeck and Benetti, 1996).

5. Other mixing processes, and wind mass loss, which affect surface composition

Mixing processes other than the standard dredge-up processes - thermal and gravitational diffusion, convective overshoot at radiative-convective boundaries, and rotation-induced mixing - can in several instances affect the surface composition of single stars. Convective overshoot has already been discussed as an important factor in the third dredge-up episode.

A pronounced Li deficiency in Hyades main-sequence stars with surface temperatures in the 6400-7000 K range (Boesgaard and Trippico, 1986) demonstrates that lithium is diffusing into the interior of the gap stars. It is possible that a combination of gravitational and thermal diffusion inward through the base of a shallow convective envelope combined with the effects of radiative levitation (Michaud, 1986) may account for a reduction in the surface $\mathrm{Li}$ abundance, but the fact that the surface abundance of lithium in subgiants in the old cluster M67 decreases with distance from the main sequence indicates that a fair fraction of the lithium diffusing inward must also be destroyed and this may imply rotationinduced mixing to temperatures large enough that lithium is destroyed (Deliyannis, King, and Boesgaard, 1996).

Wind mass loss can also influence surface composition. For example, a radiative wind may remove the hydrogen-rich surface layer of a massive enough postAGB star and possibly the remnant helium layer as well (Iben and Tutukov, 1996), and particle diffusion acts in white dwarfs of all masses to cause all but the lightest isotopes remaining in the white dwarf to settle below the photosphere. On passing through a molecular cloud, a white dwarf accretes hydrogen-rich material and this complicates the interpretation of the observed surface abundance distribution. Several of these processes are probably contributing to the observed variation in the ratio of DA white dwarfs (hydrogen-rich spectrum) to non-DA white dwarfs (hydrogen-deficient spectra) as a function of luminosity (MacDonald, 1992).

\section{Evolution of massive single stars that produce neutron stars or black holes}

Massive stars which ultimately explode as supernovae, leaving behind a relativistic remnant (NS or $\mathrm{BH}$ ), are responsible for most of the elements heavier than helium produced in the Galaxy. Lighter stars contribute primarily carbon, nitrogen, and $s$-process isotopes. One of the major features of massive star evolution prior to the supernova explosion is mass loss via a strong radiative wind (Cassinelli, 1979). The mass-loss rate increases with increasing initial mass and luminosity and continues as the star evolves, with mass-loss rates becoming as large as several times $10^{-5} M_{\odot} \mathrm{yr}^{-1}$. For the initially most massive stars, say $\widetilde{ } \widetilde{ } 30 M_{\odot}$, the mass-loss time scale is comparable to or shorter than the nuclearburning lifetime and, in constructing models to compare with the observations, it is absolutely essential to take mass loss into account. Work along these lines prior to 1986 is reviewed by Chiosi and Maeder (1986). More recently, extensive grids of theoretical models using Livermore opacities (Iglesias and Rogers, 1996) and incorporating mass loss according to various algorithms have been constructed by several groups for a variety of initial compositions (e.g., Schaller et al., 1992; Schaerer et al., 1992, 1993; Charbonnel et al., 1993; Bressan et al., 1992; Stothers and Chin, 1992, 1993a, 1993b).

The most massive stars eventually lose their hydrogen-rich envelopes and expose, first, matter which has experienced complete hydrogen burning and, then, matter that has experienced partial helium burning. These "stripped" stars are known collectively as WolfRayet (WR) stars and are subdivided into the WN class ( $\mathrm{He}$ and $\mathrm{N}$ in the spectrum) and the WC class (He, C, and $\mathrm{O}$ in the spectrum) (Abbott and Conti, 1987). In an important study of the structure of WR stars, Langer (1989a, 1989b) compares models with the observations to determine that the core mass $M_{\mathrm{WR}}$ of WR stars falls in the range $5 M_{\odot}<M_{\mathrm{WR}}<20 M_{\odot}$, independent of initial mass, that the mass-loss rate can be approximated by $\dot{M}_{\mathrm{WR}}=(0.6-1.0) \times 10^{-7}\left(M_{\mathrm{WR}} / M_{\odot}\right)^{2.5} \quad M_{\odot} \quad \mathrm{yr}^{-1}$, and that the luminosity $L_{\mathrm{WR}}$ satisfies $4.8<\log L_{\mathrm{WR}} / L_{\odot}<5.5$. The photosphere of WR stars lies in the escaping wind, and Langer finds the edge of the core to be typically at optical depth $\sim 10$. Finally, he finds that WN stars evolve from main-sequence progenitors of initial mass much smaller than the initial mass of WC progenitors.

The absence of all but a handful of stars in the Galaxy with luminosities greater than $\sim 10^{6} L_{\odot}$ and with surface temperatures less than $T_{\mathrm{e}} \sim(1-3) \times 10^{4}$ (Humphreys, 1978, Humphreys and Davidson, 1979, 1994) has been argued by De Jager (1984) to be the consequence of an instability in the photosphere, which sets in for stars brighter than the observed luminosity limit. The instability leads to dissipation of mechanical energy and the development of supersonic turbulent motions, which re- 
sult in a dramatic increase in the mass-loss rate with increasing luminosity. Stothers and Chin (1993a, 1993b, 1996) suggest another mechanism of enhanced mass loss involving a classical ionization instability in the stellar envelope. In both cases, enhanced mass loss inhibits evolution into the Humphreys-Davidson forbidden zone. Whatever the source of the instability, the observations show that single stars initially more massive than $\sim 50 M_{\odot}$ do not become giants before experiencing a supernova explosion, and this has important ramifications for massive close binary star evolution.

It is clear that massive stars interact with the interstellar medium both as energy sources and sources of isotope enrichment prior to the supernova explosion that terminates their lives. Some aspects of this interaction are explored in a recent volume by Kunth et al. (1997). Models of Galactic chemical evolution (e.g., Pagel, 1989; Matteuchi, 1989; and Taylor, 1990) rely heavily on theoretical estimates of the nucleosynthetic yields of massive stars (e.g., Maeder, 1992; Timmes et al., 1996; Thielemann et al., 1986; Arnett 1996), but uncertainties still remain (Arnett, 1995), particularly with regard to the extent of mass loss prior to the supernova explosion and with regard to the dividing line in initial mass between those stars which form BHs and therefore do not return iron-peak elements to the interstellar medium and those which form NSs and do (Maeder, 1992; Timmes et al., 1996).

After the core carbon-burning phase, the chemically evolved interior of massive stars follows one of two evolutionary paths, depending on the initial stellar mass and composition. For solarlike initial composition, models of initial mass in the 11-13 $M_{\odot}$ range experience core collapse initiated by electron capture before they form an "Fe-Ni" core (see below); in more massive models, core collapse is initiated after the formation of the $\mathrm{Fe}-\mathrm{Ni}$ core. A model star of mass $11 M_{\odot}$ is just at the borderline between stars that become TPAGB stars with stable electron-degenerate cores of $\mathrm{ONe}$ and stars that immediately form electron-degenerate $\mathrm{ONe}$ cores massive enough that electron captures on ${ }^{20} \mathrm{Ne},{ }^{23} \mathrm{Na}$, and ${ }^{24} \mathrm{Mg}$ trigger a rapid contraction that cannot be halted even by explosive O and Ne burning (Miyaji et al., 1980; Nomoto, 1984, 1987; Miyaji and Nomoto, 1987; Gutierrez et al., 1996). Nuclear reactions convert the composition of the core into iron-peak isotopes, and the Fe-Ni core collapses dynamically to nuclear matter densities. The real analog of the $11 M_{\odot}$ model and real analogs of models slightly less massive (say $\left\ulcorner 10.75 M_{\odot}\right.$ ) probably live long enough as AGB stars that the ONe core becomes massive enough and therefore dense enough to evolve into a NS (Nomoto, 1987).

Model stars more massive than $\sim 13 M_{\odot}$ burn carbon, neon, oxygen, and silicon quiescently and then form a core of iron-peak isotopes in statistical equilibrium (see Clayton, 1968; Arnett 1996; and Meyer, this review, Sec. $\mathrm{XV}$ ). Beyond the core carbon-burning phase (e.g., the third heavy portion along the $25 M_{\odot}$ track in Fig. 1), there is essentially no motion in the H-R diagram; the rapidly evolving core and the hydrogen-rich envelope are essentially decoupled. Contraction and heating of the Fe-Ni core leads to partial photodisintegration of iron-peak isotopes into alpha particles and neutrons (B ${ }^{2} \mathrm{FH}$; Hoyle and Fowler, 1960; Fowler and Hoyle, 1964). Details of the subsequent dynamical collapse of the core, neutronization, trapping of neutrinos, core bounce, and expulsion of the envelope in a type II supernova (SNII) explosion are described by many authors (e.g., Woosley and Weaver 1986; Arnett, 1996). The supernova ejecta contains ${ }^{56} \mathrm{Ni}$, which beta decays into ${ }^{56} \mathrm{Co}$, which in turn beta decays into ${ }^{56} \mathrm{Fe}$. Associated gamma emission from the decay of excited nuclear levels helps power the light curve. Analysis of the light curve of SN1987a in the Large Magellanic Cloud (Arnett et al., 1989 and references therein) suggests that $\sim 0.1 M_{\odot}$ of ${ }^{56} \mathrm{Fe}$ is ejected into the interstellar medium when a star of initial mass $\sim 20 M_{\odot}$ explodes, showing that SNeII are potent sources of iron in the Universe. That they must also be major sources of other heavy elements follows from detailed models of explosions set off in the envelopes of evolutionary models that have been carried to the stage of $\mathrm{Fe}-\mathrm{Ni}$ core collapse (e.g., Thielemann et al., 1996, and references therein).

Despite much effort expended over the past 40 years, an unambiguous theoretical picture of the detachment of the neutronized, lepton-degenerate core has not yet emerged, although the evolution of the final detached core is reasonably well understood (Burrows and Lattimer, 1986, 1987). The details of how energy is transferred from the core in such a way as to cause expulsion of the stellar envelope and a quantitative estimate of how much matter falls back onto the core are not yet known theoretically. Thus, a secure theoretical mapping between initial main-sequence mass and final NS or BH mass is not presently available (although, see Timmes et al., 1996 for an encouraging effort), and the critical initial mass $M_{\text {crit }}$ which separates stars that form NS remnants from those that form $\mathrm{BH}$ remnants is not known. However, an understanding of massive close binary evolution requires this information. In order to make progress, a concrete choice must be made, and, in the following, it will be assumed that $M_{\text {crit }}=40 M_{\odot}$, $M_{\mathrm{NS}}=1.4 M_{\odot}$, and $M_{\mathrm{BH}}=10 M_{\odot}$. The choice of $1.4 M_{\odot}$ for the gravitational mass of a typical neutron star is not inconsistent with the average value of $1.35 \pm 0.27$ estimated by Thorsett et al. (1993) for 17 NSs in binary systems.

Historically, supernovae have been classified primarily on the basis of their spectral features, with type I $\mathrm{SNe}$ (SNeI) being hydrogen deficient and SNeII exhibiting hydrogen lines. The SN light curve has in recent years become important in identifying the nature of the explosion. Comparison between spectral features and light curves of theoretical models of explosions (see, e.g., Wheeler et al., 1995) suggest that SNeII are the end result of the evolution of massive stars that retain their hydrogen-rich envelopes and explode as red or blue supergiants (e.g., SN1987A), and that SNeIb,c are the result of the evolution of stars that become WR stars before exploding. In close binaries, the primary may 
become a WR star in consequence of Roche lobe overflow, enlarging the range in initial mass of stars that produce $\mathrm{SNeIb}, \mathrm{c}$. SNeIa may involve the explosion of $\mathrm{CO}$ white dwarfs in close binary systems.

\section{Close binary star evolution}

Approximately half of all binary stars are at large enough initial orbital separation that each component evolves essentially as a single star for its entire life. The other half interact by mass transfer and/or mass loss from the system and evolve into single stars (in consequence of mergers), sometimes with unusual surface compositions (e.g., R CrB and other hydrogen deficient stars), or into exotic binary systems with interesting and often bizarre characteristics such as cataclysmic variables, $\mathrm{x}$-ray binaries, and close white dwarf pairs.

1. Modes of mass transfer and of orbital angular momentum loss

For a binary in a circular orbit, when the gravitational potential is calculated in the rotating frame on the assumption that both components are point masses, there is a unique equipotential surface consisting of two "Roche" lobes (forming an "hour-glass" with a figureeight cross section) which touch at a point along the line joining the stellar centers. The radius of a sphere having the same volume as a Roche lobe is known as the Roche lobe radius. When, in the course of evolution, the radius of a binary component approaches and exceeds its Roche lobe radius, mass is transferred from the "Roche lobe filling" star to its companion.

When the two components are of comparable initial mass and the Roche lobe filling star does not possess a deep convective envelope, mass is transferred on a time scale which is comparable to or longer than the thermal time scale of the accreting component. Under these conditions, mass is transferred "conservatively" (no mass is lost from the system) and, when the mass of the accretor exceeds that of the donor, orbital angular momentum conservation requires that the orbital separation $A$ increases with continued mass transfer.

When the donor is initially considerably more massive than its companion (by, say, a factor of 2 or more) and/or if it possesses a deep convective envelope, mass is transferred initially so rapidly that the accretor cannot adjust its structure to accomodate the proferred mass, which forms an expanding hot blanket about the accretor. The transferred matter soon fills the Roche lobe of the accretor and the situation can thereafter be described in terms of a "common envelope" (CE) (Paczyński, 1976) which consists of matter which has been supplied by the donor. The matter in the CE is driven away from the system by an "egg beater" frictional interaction between the imbedded stars and the CE material. The energy required to drive off $\mathrm{CE}$ material derives from the orbital binding energy and the orbit shrinks. The efficiency of the $\mathrm{CE}$ mechanism may be defined by $\alpha_{\mathrm{CE}}=\Delta E_{\text {remove }} / \Delta E_{\text {bind }}$, where $\Delta E_{\text {remove }}$ is the energy required to remove all of the $\mathrm{CE}$ matter from the system and $\Delta E_{\text {bind }}$ is the difference between the final and the initial orbital binding energy. The smaller $\alpha_{\mathrm{CE}}$, the greater is the degree of orbital shrinkage. The value of $\alpha_{\mathrm{CE}}$ appropriate for different situations has been debated for several decades (see, e.g., Iben and Livio, 1993, and references therein), but recent threedimensional smooth particle hydrodynamic calculations suggest a value near unity (Razio and Livio, 1996; Yorke et al., 1995).

Roche lobe filling can be achieved in consequence of the radius increase brought about by the nuclear evolution of a potential donor (e.g., Webbink et al., 1983; Taam, 1983a), or in consequence of orbital angular momentum loss due to gravitational wave radiation (GWR) (Kraft et al., 1962; Paczyński, 1967; Faulkner, 1971), or due to a magnetic stellar wind (MSW) (e.g., Verbunt and Zwaan, 1981; Taam, 1983b). In order for GWR to be important, $A$ must be less than $2-3 R_{\odot}$ (depending on component masses). In order for a MSW to be effective, the donor or the accretor must be a main-sequence star in the approximate range $0.3-1 M_{\odot}$. Reviews of evolutionary processes in binary star evolution include Paczyński (1971b) and Bhattacharya and van den Heuvel (1991).

\section{Scenario modeling}

With the help of the properties of evolving single stars described in Secs. III.B and III.C, and using the principles just mentioned, one can construct scenarios for the evolution of binary systems consisting initially of main-sequence stars for various choices of the mass $M_{10}$ of the primary, the mass ratio $q_{0}=M_{20} / M_{10}$, the orbital semimajor axis $A_{0}$, and the eccentricity $e_{0}$.

An approximation to the birthfunction for stars in the Galactic disk which is based on an analysis of the properties of many binary systems in the literature (Kraicheva et al., 1978; Popova et al., 1982) is (Iben and Tutukov, 1984)

$$
d^{3} \nu\left(\mathrm{yr}^{-1}\right)=0.2 d \log A_{0} \frac{d M_{10}}{M_{10}^{2.5}} d q_{0}
$$

where $M_{10}$ and $A_{0}$ are in solar units. Integrating Eq. (1) over $A_{0}=10-10^{6}, M_{10}=0.8-100$, and $q_{0}=0-1$ gives $\nu \sim 1 \mathrm{yr}^{-1}$ as the Galactic birthrate of stars that leave the main-sequence in less than a Hubble time.

When mass transfer is deemed to be conservative, conservation of orbital angular momentum gives

$$
J_{\text {orb }}=M_{1 \mathrm{f}} M_{2 \mathrm{f}}\left(\frac{G A_{\mathrm{f}}}{M_{\mathrm{t}}}\right)^{1 / 2}=M_{10} M_{20}\left(\frac{G A_{0}}{M_{\mathrm{t}}}\right)^{1 / 2},
$$

where the subscript $\mathrm{f}$ identifies system parameters after the mass transfer event is completed and $M_{\mathrm{t}}=M_{10}+M_{20}$ $=M_{1 \mathrm{f}}+M_{2 \mathrm{f}}$. When CE evolution is invoked, one must adopt some approximations for $\Delta E_{\text {remove }}$ and $\Delta E_{\text {bind }}$ in the definition of $\alpha_{\mathrm{CE}}$. For example, for large $q_{0}$, a rough approximation is

$$
\alpha_{\mathrm{CE}} \sim\left(\frac{G M_{10}^{2}}{A_{0}}\right) /\left(\frac{G M_{1 \mathrm{f}} M_{20}}{A_{\mathrm{f}}}\right)=\frac{M_{10}}{M_{20}} \frac{M_{10}}{M_{1 \mathrm{f}}} \frac{A_{\mathrm{f}}}{A_{0}} .
$$


Since both $M_{20}$ and $M_{1 \mathrm{f}}$ are small compared to $M_{10}$, it is clear that orbital shrinkage can be considerable in a CE event. Equations (1)-(3), in conjunction with properties of evolving stellar models (including externally imposed mass loss at a high rate), have been used to reproduce (with $\alpha_{\mathrm{CE}} \sim 1$ ) empirically estimated birthrates of a variety of evolved binary systems in the Galactic disk (e.g., Yungelson and Tutukov, 1991; Tutukov and Yungelson, 1992; Tutukov et al., 1992; Yungelson and Tutukov, 1993; Iben, Tutukov, and Yungelson, 1997).

\section{a. Cataclysmic variables and novae}

Cataclysmic variables (CVs) are white dwarfs with a low mass companion that is typically a main-sequence star that fills its Roche lobe (see Warner, 1995, and references therein). In systems with orbital periods in the 1.3-2 $\mathrm{h}$ range, mass transfer is driven by angular momentum loss due to GWR at a rate $(1-2) \times 10^{-10} M_{\odot}$ $\mathrm{yr}^{-1}$. In CVs with periods in the range 3-20 h, mass transfer is driven by a MSW at a rate in the range $10^{-9}$ $10^{-8} M_{\odot} \mathrm{yr}^{-1}$. Systems born into the long period category evolve to shorter periods as mass is transferred until $P_{\text {orb }} \sim 3 \mathrm{~h}$, whereupon the main-sequence component becomes completely convective and no longer supports a MSW. The mass transfer rate drops abruptly, causing the donor to shrink within its Roche lobe and mass transfer to cease. Mass transfer begins again when, in consequence of GWR, the orbital separation has decreased to the extent that the main-sequence star again fills its Roche lobe.

The hydrogen-rich matter supplied by the donor is stored in a disk and transferred from there to the white dwarf, sometimes in a sporadic discharge that produces a flare up called a dwarf nova outburst. The light emitted in such an outburst is due to the release of gravitational potential energy. In the absence of differential rotation, gravitational and thermal diffusion would mix hydrogen-rich accreted material with white dwarf material near the base of the accreted layer (Prialnik and Kovetz, 1984). However, matter from the disk reaches the surface of the white dwarf with velocities near the Keplerian velocity at the surface of the WD and angular momentum diffuses inward through the accreted layer and beyond, creating a radial gradient in the angular velocity. Differential rotation gives rise to a baroclinic instability (Fujimoto, 1988, 1993) which causes even greater mixing between accreted and white dwarf material than does particle diffusion (Fujimoto and Iben, 1997). When the white dwarf has accreted enough material (e.g., $\sim 10^{-5} M_{\odot}$ when $\dot{M}_{\mathrm{WD}} \sim 10^{-9} M_{\odot} \mathrm{yr}^{-1}$ ), hydrogen is ignited near the base of the accreted layer and a convective zone extends inward (to the point in the mixing region where the abundance by mass of hydrogen is initially $\sim 0.01$ ) and outward (to the surface), mixing large quantities of $\mathrm{CO}$ or $\mathrm{ONe}$ material outward. Conversion of nuclear energy into heat lifts the electron degeneracy in the hydrogen-rich layer, and enough energy is injected to force this layer to expand to giant dimensions (e.g., to the right along the dash-dot curve in
Fig. 1 found in a model calculation that neglects the companion [Iben, 1982]). In the real analog, called a classical nova, the expanding matter extends far beyond the Roche lobe of the white dwarf and a combination of CE action (e.g., MacDonald, 1980, 1986; Livio et al., 1990), wind mass loss (e.g., Kato, 1983; Kato and Hachisu, 1994), and, in some cases, dynamical acceleration (e.g., Starrfield et al., 1974, 1978) removes most of the expanding layer. The remnant hydrogen-rich, heavy element rich layer contracts onto the white dwarf and, in model calculations, the star moves to the left in the H-R diagram in Fig. 1 along the dash-dot track at radii smaller than $\sim 1 R_{\odot}$.

Equations (1)-(3), with $\alpha_{\mathrm{CE}} \sim 1$, have been used to estimate that $\mathrm{CVs}$ are born at the rate $\sim 0.001 \mathrm{yr}^{-1}$ (Iben and Tutukov, 1984). When observational selection effects are taken into account, this is not inconsistent with the birthrate estimated from the observed distribution of CVs in the solar vicinity (Trimble, 1982). The relative numbers of short period and long period CVs can be understood in terms of the time scales for mass transfer driven by GWR and a MSW and by observational selection effects (e.g., Iben et al., 1991).

The maximum brightness of a classical nova is directly related to the mass of the white dwarf according to the relationship $L_{\max } / L_{\odot}=46,000\left(M_{\mathrm{wD}} / M_{\odot}-0.26\right)$ (Iben and Tutukov, 1989, 1996a), which, for $M_{\mathrm{WD}} \widetilde{\sim} M_{\odot}$, is similar to the Paczyński-Uus (Paczyński, 1970; Uus, 1970) relationship, $L_{\max } / L_{\odot}=59,000\left(M_{\mathrm{WD}} / M_{\odot}-0.52\right)$, that is applicable to central stars of PNe. The difference between the two relations is due to the fact that the underlying white dwarf in the nova case is colder and therefore has a smaller radius than in the PN case.

The dependence of $L_{\max }$ on $M_{\mathrm{WD}}$ provides a strong observational bias for novae with massive white dwarf cores and explains why the progenitor of a typical observed nova is a main-sequence pair with a fairly massive primary. The requirement that the main-sequence donor be less massive than the white dwarf by a factor of $\sim 2 / 3$ (to ensure stable mass transfer) means, then, that $q_{0} \ll 1$. This, in turn, means that mass transfer in the progenitor binary is such that a $\mathrm{CE}$ is surely formed and that orbital shrinkage will, in many instances, force the main-sequence component to be drawn by a MSW or GWR into Roche lobe contact in less than a Hubble time.

\section{b. White dwarf mergers: $R$ CrB stars and type la supernovae}

R Corona Borealis ( $\mathrm{R} \mathrm{CrB}$ ) stars and SNeIa are most easily explained in terms of the merger of two white dwarfs. In the case of SNeIa, one of the most compelling arguments for implicating binary stars is the fact that SNeIa occur in elliptical galaxies that are primarily made up of old stars that are not by themselves massive enough to become SNe. SNeII do not occur in elliptical galaxies, showing that the formation of massive stars is not now taking place, and various observations tell us that, in our own Galaxy, single stars less massive than $\sim 10 M_{\odot}$ evolve into white dwarfs. 
A natural way of accounting for these facts is to suppose that, during an earlier period of active star formation, two fairly massive intermediate mass mainsequence stars evolve through two CE episodes into a pair of white dwarfs of total mass larger than the Chandrasekhar mass $\left(\sim 1.4 M_{\odot}\right)$ and at a separation small enough that angular momentum loss due to GWR brings the two together in less than a Hubble time until a merger followed by an explosion occurs (Iben and $\mathrm{Tu}-$ tukov, 1984; Webbink, 1984). The details of how this comes about are not resolved (see, e.g., Iben, 1997). If, when the less massive white dwarf overfills its Roche lobe, the mass ratio of the white dwarfs is less than $2 / 3$, mass transfer is stable (Tutukov and Yungelson, 1979). Otherwise, the less massive white dwarf is transformed on a dynamical timescale into a thick disk around the more massive white dwarf (Tutukov and Yungelson, 1979; Iben and Tutukov, 1984; Webbink and Iben, 1987; Benz et al., 1990; Mochkovich and Livio, 1990). If the angular momentum in the disk cannot diffuse outward and be carried away in a wind rapidly enough (Mochkovich and Livio, 1990), carbon burning may convert the interior into an ONe composition (Nomoto and Iben, 1985; Saio and Nomoto, 1985) and the final outcome may be collapse into a neutron star. If angular momentum can diffuse outward fast enough, a collapse will be followed by a star disrupting explosion, which converts much of the star into iron-peak elements moving with velocities of $\sim 10^{4} \mathrm{~km} \mathrm{~s}^{-1}$, similar to those observed for SNeIa explosions.

There is an abundance of theoretical calculations showing that, when a thermonuclear explosion is set off at the center of a $\mathrm{CO}$ white dwarf model, the resultant spectral distribution reproduces many of the observed features of SNIa spectra (e.g., Nomoto et al., 1984,1997), the theoretical light curves can be made to match observed light curves by varying parameters in the theory (e.g., Höflich and Khokhlov, 1996; Höflich et al. 1996), and the isotope mix produced in the explosion can help explain the abundances of several isotopes that are not produced in supernovae of other types (e.g., Thielemann et al., 1997). Most extant simulations of SNeIa explosions do not address the physics of the merger process, but assume rather that the CO WD accretes matter quiescently from a presumably hydrogen-rich companion. However, the merger configuration is highly nonspherical, carbon may ignite off center in many places at the same time, and the physics of burning under turbulent conditions is complex (e.g., Niemeyer et al., 1996). It will be interesting to see what new features are revealed when three-dimensional models of the merging process are constructed with adequate resolution and realistic physics.

On the scenario front, the estimate of $0.003 \mathrm{yr}^{-1}$ for the formation frequency of double $\mathrm{CO}$ white dwarfs in our Galaxy, which have a total mass larger than $1.4 M_{\odot}$ and which are close enough to merge in a Hubble time (Tutukov et al., 1992), is almost identical with empirical estimates of the SNIa birthrate (Baade and Minkowski, 1957; van den Bergh et al., 1987; van den Bergh and
Tammann, 1991). On the observational front, eight systems consisting of two close white dwarfs have been discovered and at least three of these pairs are at an orbital separation such that a merger will occur in less than a Hubble time. In most of the observed systems, the visible star is a helium WD. The distribution in number versus period of the observed systems is consistent with scenario predictions, and it is predicted that the observed sample of close binary WDs must be increased by at least a factor of 4 before the merging CO WD scenario for SNeIa begins to be seriously challenged or possibly strengthened (Iben, Tutukov, and Yungelson, 1997).

Other possible progenitors of SNeIa include supersoft $\mathrm{x}$-ray sources that are thought to be white dwarfs accreting matter at a high rate from a Roche lobe filling mainsequence star of mass 1-2 $M_{\odot}$ (van den Heuvel et al., 1992; Di Stefano et al., 1997). The high rate of mass transfer prevents nova explosions, but wind mass loss from the system is important and the hydrogen-rich matter in the system must somehow be hidden if these systems evolve into SNeIa.

$\mathrm{R}$ CrB stars may also be explicable in terms of binary star evolution (Webbink, 1984; Iben and Tutukov, 1984a, 1985; Iben and Tutukov, 1996a, 1996b). They are bright $\left(\langle L\rangle \sim 7 \times 10^{3} L_{\odot}\right)$ and cool (average $T_{\mathrm{e}} \sim 7,000$ $\mathrm{K})$ with extreme hydrogen-deficient spectra and strong overabundances of carbon (Bidelman, 1953) and nitrogen (Rao and Lambert, 1996, and references therein). Single star models of the born-again variety, while developing appropriately exotic surface compositions (Renzini, 1990; Iben and MacDonald, 1995), possibly do not live long enough as cool bright stars to account for most of the estimated 200-1000 R CrB stars in the Galaxy (Lawson et al., 1990). The problem is that the amount of fuel available for nuclear burning is inversely proportional to a high power of the mass of the underlying white dwarf core but the luminosity and radius achievable are directly proportional to core mass.

In the binary scenario, the immediate precursor of an $\mathrm{R}$ CrB star is a CO WD and a He WD at a separation small enough that a merger due to GWR will occur in less than a Hubble time. The idea is that, when it fills its Roche lobe, the lighter He WD will spill over onto the $\mathrm{CO} \mathrm{WD}$, and turbulent convective motions will mix carbon and oxygen from the CO WD into the helium and nitrogen of which the $\mathrm{He}$ WD is composed. Helium burning at the base of the helium-rich convective envelope of the merger product increases the mass of the $\mathrm{CO}$ core and, if the mass of the merger product is large enough $\left(\widetilde{\sim} 0.78 M_{\odot}\right)$, the star may evolve into the domain in the H-R diagram occupied by $\mathrm{R} \mathrm{CrB}$ stars when the $\mathrm{CO}$ core mass exceeds $\left(\sim 0.56-0.61 M_{\odot}\right)$.

The fuel available in the helium envelope for burning at high luminosity is $\sim 20$ times larger than in the bornagain scenario, and the predicted presence of 200-600 bright and cool systems in the Galaxy at any given time is consistent with the observational estimate of 2001000. The most important task in pursuing the binary scenario is the construction of 3-D hydrodynamic mod- 
els of the merging process to see if compositional mixing occurs to the degree necessary to explain the observed $\mathrm{R}$ $\mathrm{CrB}$ compositions. The technology for exploring this question is advancing rapidly (e.g., Bazan and Arnett, 1994; Burrows et al., 1995; Livne and Arnett, 1995; Glasner and Livne, 1995) and one may anticipate resolution of this question in the not too distant future. On the observational and scenario fronts, there are two known white dwarf pairs (WD 2331+290 and WD 0957-666) that will merge in less than a Hubble time and that have mass estimates that do not preclude evolution into the configurations envisioned here (Iben, Tutukov, and Yungelson, 1997).

\section{c. X-ray binaries and pulsars}

Approximately half of all massive stars are in binaries close enough that the primary fills its Roche lobe and loses its hydrogen-rich envelope before developing an Fe-Ni core that evolves into a NS or BH. The nucleosynthetic yield of such stars is quite different from that of single massive stars, and this difference must be taken into account in constructing models of Galactic nucleosynthesis. Furthermore, much of what we know about NSs and BHs comes from a study of close binaries in which at least one of the components is an accreting NS or BH (Novikov and Zeldovich, 1966; Shklovski, 1967).

Low mass x-ray binaries (LMXBs) consist of a NS or $\mathrm{BH}$ and either a low mass main-sequence donor or a subgiant donor with an electron-degenerate helium core (Bhattacharya and van den Heuvel, 1991, and references therein). The donor transfers matter to the relativistic component because it either fills or is close to filling its Roche lobe. Conventional wisdom has it that the donor fills its Roche lobe and that mass transfer is driven by angular momentum loss due to a MSW (if the donor is a main-sequence star) or by radial expansion of the donor due to interior nuclear transformations (if the donor is a subgiant). In this picture, the mechanisms of mass transfer in a CV and in an LMXB are identical and differences between the two types of system stem primarily from the huge difference (factor of $\sim 1000$ ) in the potential energy of a particle at the surface of the relativistic component in an LMXB relative to that of a particle at the surface of the WD in a CV. In an LMXB, the nuclear energy liberated in a thermonuclear outburst is a small fraction of the potential energy liberated in the process of accretion between thermonuclear outbursts; in a $\mathrm{CV}$, the reverse is true. Thus, an LMXB is most conspicuous when an accretion disk discharges onto the relativistic component, whereas a $\mathrm{CV}$ is most conspicuous during a classical nova outburst when the luminosity is due to the release of nuclear energy.

In Fig. 1, the maximum luminosity (van Paradijs, 1995) of a number of LMXBs with NS accretors for which distances have been estimated (van Paradijs and White, 1995) are shown along a line of constant radius $\left(10^{-5} R_{\odot}\right)$ to provide an indication of the large temperatures characterizing the observed spectrum. Shown also are the maximum luminosities of LMXBs with BH accretors according to Tanaka and Lewin (1995).
The progenitor of an LMXB is distinguished from that of a CV progenitor by the magnitude and nature of the hurdles that it must surmount (van den Heuvel, 1985; Webbink and Kalogera, 1994). The net result is that the theoretical birthrate of LMXBs with mainsequence or subgiant donors and NS accretors is quite small. For example, one estimate of this birthrate is $\nu \sim 2 \times 10^{-5} \mathrm{yr}^{-1}$ when $\alpha_{\mathrm{CE}}=1$ and $\sim 2 \times 10^{-6} \mathrm{yr}^{-1}$ when $\alpha_{\mathrm{CE}}=0.5$ (Iben and Tutukov, 1995). With the choices $M_{\mathrm{BH}}=10 M_{\odot}$ and $40<M_{10} / M_{\odot}<50$, systems with $\mathrm{BH}$ accretors are born approximately $20 \%$ as frequently as the corresponding systems with NS accretors.

The NS in an LMXB accretes mass from the base of an accretion disk at essentially the Keplerian velocity at the surface of the NS (Alpar et al., 1982), causing the NS to spin up until the counter torque exerted by a magnetic field coupled to ionized accreted matter balances the torque supplied by the accreted matter (Gosh et al., 1977; Chen and Ruderman, 1993). Accretion of only $\sim 0.01 M_{\odot}$ is sufficient to decrease the spin period into the millisecond range $\left(\approx 10 \times 10^{-3} \mathrm{~s}\right)$. In LMXBs with main-sequence donors, the donor is probably destroyed by absorption of particle and photon emission from the rapidly spinning NS, which then evolves into a single millisecond pulsar (MSP). In LMXBs with subgiant donors, the electron-degenerate core survives as a helium WD and the final system is a binary MSP. Comparison between a measured beat period and the Keplerian period permits one to infer the spin period of the NS in an LMXB (Alpar and Shaham, 1985; Lamb et al., 1985; Gosh and Lamb, 1992), and this has been accomplished in three LMXBs (Strohmayer et al., 1996; Morgan and Smith, 1996; Ford et al., 1997).

High mass x-ray binaries (HMXBs) consist of a NS or $\mathrm{BH}$ which accretes from the radiative wind emitted by a massive OB star which does not fill its Roche lobe (van den Heuvel and Heise, 1972; Tutukov and Yungelson, 1973). In many instances, the main-sequence components in the primordial binary are of comparable mass, so that the first mass transfer event is conservative, with the orbital separation of the binary increasing during the transfer and the secondary being spun up in the process (Rappaport and van den Heuvel, 1982).

Because the secondary in the progenitor system is massive to begin with, and the primary evolves into a helium star much less massive than the secondary, mass loss during the SNIb,c explosion of the helium star does not in general disrupt the binary system. The duration of the bright $\mathrm{x}$-ray stage is but a small fraction of the mainsequence lifetime of the donor (Massevich et al., 1979; Savonije, 1979), and this accounts for the observed paucity of bright HMXBs (there may be only $\sim 100$ with $L_{\mathrm{X}}>10^{3} L_{\odot}$ in the Galaxy).

Eventually, the OB component in an HMXB evolves to fill its Roche lobe, lose most of its hydrogen-rich envelope in a CE event, and explode as a SNIb,c. The binary system is, in general, disrupted. The typical final result is two high velocity NSs with spin rates of $\sim 3 \mathrm{~s}$ or 
less. Of course, some few remain bound, as is demonstrated by the famous binary pulsar 1913+16 (Hulse and Taylor, 1975).

NSs formed in SNIb,c explosions in close binaries may be the origin of most pulsars with spin period in the $0.01-3 \mathrm{~s}$ range. The first formed NS has a modest spin rate because it was spun up by accretion from its $O B$ companion. The second NS has a modest spin rate because its progenitor was spun up during the first mass transfer episode. The birthrate of all SNeIb,c explosions in the Galaxy is predicted to be $\sim 0.007 \mathrm{yr}^{-1}$ (Tutukov and Yungelson 1993), compared with a theoretical birthrate of $\sim 0.005 \mathrm{yr}^{-1}$ for NSs formed by systems which pass through the HMXB stage. These estimates are consistent with a semiempirical estimate of the pulsar birthrate of 0.004-0.008 $\mathrm{yr}^{-1}$ (Lorimer et al., 1993). On the other hand, the predicted birthrate of NSs formed in SNII explosions of massive single stars (including those formed by a merger in a first $\mathrm{CE}$ event) and massive stars in wide binaries is $\sim 0.021 \mathrm{yr}^{-1}$ (Tutukov and Yungelson, 1993), which agrees with the empirical estimate of the SNII birthrate (Baade and Minkowski, 1957; van den Bergh and Tammann, 1991). Thus, one could infer that pulsars are produced only by close binaries.

\section{HYDROGEN BURNING IN THE $p p$ CHAIN AND CN CYCLE}

In 1957, the energetics of $4{ }^{1} \mathrm{H} \rightarrow{ }^{4} \mathrm{He}$ fusion were well established, and the two sequences for accomplishing that fusion (the carbon-nitrogen cycle and the protonproton chain) were laid out. The rates of each of the four reactions of the $\mathrm{CN}$ cycle had been measured directly (down to energies of $\approx 100 \mathrm{keV}$ ) to determine extrapolated values of $S(0)$ with reported precisions of 15 to $30 \%$. But from the perspective of 1997 , it may be hard to realize what a mere thimbleful of information about the rates of the reactions in the $p p$ chain was available to $\mathrm{B}^{2} \mathrm{FH} 40$ years ago. The rate of the $p\left(p, e^{+} \nu_{e}\right) d$ reaction (and hence the rate of the $p p$ chain) was $30 \%$ too small, as a result of uncertainties in the weak-interaction coupling constants. (Measurements of the related lifetime of the neutron were $25 \%$ too long.) Initial measurements of the rate of the termination reaction, ${ }^{3} \mathrm{He}\left({ }^{3} \mathrm{He}, 2 p\right){ }^{4} \mathrm{He}$, would turn out to be a factor of 4 too small (due to a problem with the ${ }^{3} \mathrm{He}$ target), and the possibility of other terminations of the $p p$ chain via ${ }^{7} \mathrm{Be}$ and ${ }^{8} \mathrm{~B}$ were not yet even discussed. All of that changed very rapidly during the next 10 years.

Now, 40 years later, we present a summary of the current status of the most important of the nuclear reaction rates in $4^{1} \mathrm{H} \rightarrow{ }^{4} \mathrm{He}$ fusion, emphasizing recent important developments and still open questions.

\section{A. The $p\left(p, e^{+} \nu_{e}\right) d$ reaction}

The rate of the $p\left(p, e^{+} \nu_{e}\right) d$ reaction is determined from the ratio of the weak interaction coupling constants, $\mathrm{G}_{A} / \mathrm{G}_{V}$, and may be related to the results of Bahcall and May (1969) as

$$
\begin{aligned}
& S_{11}(0 ; \delta) \\
& =S_{11}(0 ; 0)_{B M 69} \frac{\Lambda^{2}}{\Lambda_{69}^{2}} \frac{\left(G_{A} / G_{V}\right)^{2}}{\left(G_{A} / G_{V}\right)_{69}^{2}} \frac{\left(f_{p p}^{R}\right)}{\left(f_{p p}\right)_{69}} \frac{\left[(f t)_{0^{+} \rightarrow 0^{+}}\right]_{69}}{\left[(f t)_{0^{+} \rightarrow 0^{+}}\right]} \\
& \quad \times(1+\delta)^{2},
\end{aligned}
$$

where $\Lambda$ is the overlap integral between the two protons and the deuteron (e.g., Bahcall and May, 1969); $\left(\mathrm{G}_{A} / \mathrm{G}_{V}\right)$ is the ratio of the axial-vector and vector weak interaction coupling constants; $\left(f_{p p}^{R}\right)$ is the phase-space factor for the $p p$ reaction, $\left(f_{p p}\right)_{69}=0.142$ and $\left(f_{p p}^{R}\right)=0.144$ (INT Workshop, 1997); [ $\left.(f t)_{0^{+} \rightarrow 0^{+}}\right]$corresponds to the strength of $0^{+}$to $0^{+}$superallowed beta decays; and $\delta$ is the fractional correction to the nuclear matrix element due to exchanges of $\pi$ and $\rho$ mesons. Since 1980, there has been a marked increase in the precision of measurements of the neutron decay parameters (the neutron lifetime, as well as the $\beta$ asymmetry parameter in the decay of polarized neutrons, and the $e-\nu$ angular correlation parameter) (e.g., Freedman, 1990; and Barnett et al., 1996).

By making use of recent values for these parameters,

$$
\Lambda^{2}=6.92( \pm 1 \%)
$$

(Kamionkowski and Bahcall, 1994),

$$
\left(G_{V} / G_{V}\right)=1.2654 \pm 0.0042
$$

(Barnett et al., 1996;

INT Workshop, 1997),

$$
\begin{aligned}
& {\left[(f t)_{0^{+} \rightarrow 0^{+}}\right]=3073.3 \pm 3.5 \quad \text { (Hardy et al., 1990), }} \\
& \delta=0.01(0.004 \leqslant \delta \leqslant 0.03)
\end{aligned}
$$

(Bahcall and Pinsonneault, 1992),

we find

$$
\begin{aligned}
S_{11}(0 ; \delta)= & 3.78 \times 10^{-25}\left(\frac{6.92}{7.08}\right)\left(\frac{1.2654}{1.2382}\right)^{2}\left(\frac{0.144}{0.142}\right) \\
& \times\left(\frac{3074}{3073.3}\right)(1+.01)^{2} \mathrm{MeV} \text { b. }
\end{aligned}
$$

Currently, the most important source of uncertainty in this value is in $\delta$. The current range in $\delta$ corresponds to a range in $\mathrm{S}_{11}(0 ; \delta)$ from $3.94 \times 10^{-25}$ to $4.15 \times 10^{-25}$ $\mathrm{MeV} b$ (i.e., $\mathrm{a}+4.0 \% /-1.3 \%$ uncertainty).

\section{B. The ${ }^{3} \mathrm{He}\left({ }^{3} \mathrm{He}, 2 p\right){ }^{4} \mathrm{He}$ reaction}

Measurements of the ${ }^{3} \mathrm{He}\left({ }^{3} \mathrm{He}, 2 p\right){ }^{4} \mathrm{He}$ reaction have recently been extended down to $E_{\text {c.m. }}=24 \mathrm{keV}$ (Krauss et al., 1987) and $E_{\text {c.m. }}=21 \mathrm{KeV}$ (Arpesella et al., 1996), well into the Gamov peak corresponding to the center of the sun; for this reaction, a temperature of $T_{6}=15.6$ corresponds to a Gamov peak at $E_{o}=22 \mathrm{keV}$ with a width $\Delta E_{o}=12 \mathrm{keV}$. The most recent of these experiments was carried out using the Gran Sasso tunnel as a cosmic-ray shield. These two data sets were measured at energies that are low enough so that interacting nuclei have a 
TABLE I. Experimental measurements of the ${ }^{3} \mathrm{He}+{ }^{4} \mathrm{He}$ reaction.

\begin{tabular}{|c|c|}
\hline $\mathrm{S}_{34}(0)(\mathrm{keV} \mathrm{b})$ & Reference \\
\hline \multicolumn{2}{|c|}{ Measurement of capture $\gamma$ rays } \\
\hline $0.47 \pm 0.05$ & Parker and Kavanagh (1963) \\
\hline $0.58 \pm 0.07$ & Nagatani et al. (1969) ${ }^{a}$ \\
\hline $0.45 \pm 0.06$ & Kräwinkel et al. (1982) b \\
\hline $0.52 \pm 0.03$ & Osborne et al. $(1982,1984)$ \\
\hline $0.47 \pm 0.04$ & Alexander et al. (1984) \\
\hline $0.53 \pm 0.03$ & Hilgemeier et al. (1988) \\
\hline \multicolumn{2}{|c|}{ Weighted mean $=0.507 \pm 0.016$} \\
\hline \multicolumn{2}{|c|}{ Measurement of ${ }^{7} \mathrm{Be}$ activity } \\
\hline $0.55 \pm 0.05$ & Osborne et al. $(1982,1984)$ \\
\hline $0.63 \pm 0.04$ & Robertson et al. (1983) \\
\hline $0.56 \pm 0.03$ & Volk et al. (1983) \\
\hline \multicolumn{2}{|c|}{ Weighted mean $=0.579 \pm 0.024$} \\
\hline
\end{tabular}

${ }_{\bar{a}}^{\mathrm{a} A s}$ extrapolated using the direct-capture model of Tombrello and Parker (1963).

${ }^{\mathrm{b}}$ As renormalized by Hilgemeier et al. (1988).

classical turning point which is near or outside the atomic radius $R_{a}$ and therefore electron-screening corrections are necessary in order to relate the laboratory (screened) measurements $\left(\sigma_{s}\right)$ to cross sections which would be measured for bare nuclei $\left(\sigma_{b}\right)$ (e.g., Assenbaum et al.., 1987).

$$
\sigma_{b}=\sigma_{s} / \exp \left(\pi \eta U_{e} / E_{\mathrm{c} . \mathrm{m} .}\right)
$$

where

$$
U_{e}=Z_{1} Z_{2} e^{2} / R_{a},
$$

and $\eta$ is the Coulomb parameter. For example, for the ${ }^{3} \mathrm{He}\left({ }^{3} \mathrm{He}, 2 p\right){ }^{4} \mathrm{He}$ reaction $\left(U_{e}=240 \mathrm{eV}\right)$ at $E_{\text {c.m. }}=22$ $\mathrm{keV}$, this amounts to a $20 \%$ correction, while for $E_{\text {c.m. }}>150 \mathrm{keV}$ the correction is $\leqslant 1 \%$. [This correction to the laboratory measurements should not be confused with the plasma screening correction (Salpeter, 1954) which must be applied in relating laboratory measurements of bare-nuclear cross sections to reaction rates in plasmas.] The results of Krauss et al. (1987) and Arpesella et al. (1996) are in agreement with each other and are consistent with earlier results at higher energies (see Bahcall and Pinsonneault, 1992, and references therein). A combination of all of these results yields a value of $\mathrm{S}_{33}(0)=5.15 \pm 0.35 \mathrm{MeV} \mathrm{b}$, where there are comparable contributions to this uncertainty from the statistical and systematic uncertainties and the uncertainty in the energy dependence of the extrapolation. It is important to note that as the Gran Sasso experiment continues, we are now rapidly approaching the time when the cross section will be measured across the entire Gamow peak; at that time the rate of this reaction in the energy range of the Gamow peak will be determined directly from the measured cross sections, and the issue of how to extrapolate from the higher-energy cross sections will no longer be important.

A low-energy ${ }^{3} \mathrm{He}+{ }^{3} \mathrm{He}$ resonance has occasionally been proposed as an explanation for the solar neutrino problem; while there have already been a number of negative indirect searches (e.g., McDonald et al., 1977, and references therein), the direct low-energy results of Krauss et al. (1987) and Arpesella et al. (1996) now set an upper limit on the energy $\left(E_{\text {c.m. }}<21 \mathrm{keV}\right)$ for any such resonance.

\section{The ${ }^{3} \mathrm{He}(\alpha, \gamma){ }^{7} \mathrm{Be}$ reaction}

The relative rates of the ${ }^{3} \mathrm{He}(\alpha, \gamma){ }^{7} \mathrm{Be}$ and ${ }^{3} \mathrm{He}\left({ }^{3} \mathrm{He}, 2 p\right){ }^{4} \mathrm{He}$ reactions determine what fraction of $p p$-chain terminations result in high-energy $\left({ }^{7} \mathrm{Be}\right.$ or $\left.{ }^{8} \mathrm{~B}\right)$ neutrinos. There are six sets of measurements of the cross section for the ${ }^{3} \mathrm{He}(\alpha, \gamma){ }^{7} \mathrm{Be}$ reaction which are based on detecting the capture gamma rays (Table I). The weighted average of those six results provides a value of $S_{34}(0)=0.507 \pm .016 \mathrm{keV} \mathrm{b}$, based on extrapolations using the calculated energy dependence for this direct-capture reaction. Separate calculations of this energy dependence based on the resonating group method (Kajino et al., 1987) and on a direct-capture cluster model (Tombrello and Parker, 1963) agree to within $\pm 1.25 \%$ and are in good agreement with the energy dependence of the measured cross sections. The energy dependence of this reaction seems to be well determined by resonating group models (as noted above) so that its extrapolation to $E=0$ seems well defined, and the only free parameter is associated with the normalization of such models to the measured data sets. While the energy dependence of these models is in good agreement with the energy dependence of the measured cross sections, it would be useful to carry out a theoretical study of how robust this energy dependence is for a wider range of models. 
In addition to the six data sets of capture gamma ray measurements, there are three sets of cross-section measurements for this reaction based on the activity of the synthesized ${ }^{7} \mathrm{Be}$ (Table I). These decay measurements have the advantage of determining the total cross section directly, but have the disadvantage that (since the source of the residual activity cannot be uniquely identified) there is always the possibility that some of the ${ }^{7} \mathrm{Be}$ may have been produced in a contaminant reaction in some way that evaded tests for it. The three activity measurements (when extrapolated in the same way as the capture gamma ray measurements) provide a value of $\mathrm{S}_{34}(0)=0.579 \pm .024 \mathrm{keV} \mathrm{b}$ which disagrees with the value based on the direct-capture gamma rays at the $3 \sigma$ level.

When the nine experiments are combined, the weighted mean is $\mathrm{S}_{34}(0)=0.533 \pm 0.013 \mathrm{keV} \mathrm{b}$, with $\chi^{2}=13.4$ for 8 degrees of freedom (d.o.f.). The probability of such a distribution arising by chance is $10 \%$, and that, together with the apparent grouping of the results according to whether they have been obtained from activation or prompt-gamma yields, suggests the possible presence of a systematic error in one or both of the techniques. An approach that gives a somewhat more conservative evaluation of the uncertainty is to form the weighted means within each of the two groups of data (the data show no indication of nonstatistical behavior within the groups), and then determine the weighted mean of those two results. Then an arbitrary but standard presciption can be adopted in which the uncertainties of the means of the two groups (and hence the overall mean) are increased by a common factor of 3.7 (in this case) to make $\chi^{2}=0.46$ for 1 degree of freedom, equivalent to making the estimator of the weighted population variance equal to the weighted sample variance. The result is an overall weighted mean of $S_{34}(0)=$ $0.53 \pm 0.05 \mathrm{keV}$ b.

\section{The ${ }^{7} \mathrm{Be}(\mathrm{p}, \gamma){ }^{8} \mathrm{~B}$ reaction}

The ${ }^{8} \mathrm{~B}$ termination of the $p p$ chain is so weak $(\sim .01 \%)$ that its details do not contribute significantly to the energy generation of the sun. However, the relatively high-energy neutrino resulting from the ${ }^{8} \mathrm{~B}$ beta decay $\left(E_{\nu}^{\max } \approx 14 \mathrm{MeV}\right)$ plays a key role in several of the solar neutrino experiments (e.g., Homestake, Kamiokande, Super-Kamiokande, and SNO), so that the rate of the ${ }^{7} \mathrm{Be}(p, \gamma){ }^{8} \mathrm{~B}$ reaction is a key to understanding the results of those experiments. There have been six direct measurements of this reaction using radioactive ${ }^{7} \mathrm{Be}$ targets. A reanalysis of the five most accurate experiments (Johnson et al., 1992; Langanke, 1994) and a renormalization to a ${ }^{7} \mathrm{Li}(d, p)$ cross section of $146 \mathrm{mb}$ (Strieder et al., 1996) determined a best value of $S_{17}(0)=20.3 \pm 2.0$ $\mathrm{eV} \mathrm{b}$ where the uncertainty includes a correction of $\sqrt{\chi^{2} / \text { d.o.f. }}=1.32$ to take into account the disagreement among the five experiments (apparently arising from unidentified systematic errors) and includes the uncertainty in the ${ }^{7} \mathrm{Li}(d, p){ }^{8} \mathrm{Li}$ cross section that was used as a normalization for all of those ${ }^{7} \mathrm{Be}(p, \gamma)^{8} \mathrm{~B}$ experiments.
More recently, at the INT Workshop on Solar Nuclear Fusion Rates (1997), a more selective analysis was used to cite the Filippone et al. (1983a, 1983b) experiment as the most reliable of the direct measurements, resulting in a recommended value of $S_{17}(0)=19_{-2}^{+4} \mathrm{eV} \mathrm{b}$.

We now appear to be on the threshold of a series of promising new experiments to measure the rate of this reaction using a variety of new techniques, such as ${ }^{7} \mathrm{Be}$ beams, new ${ }^{7} \mathrm{Be}$ target strategies, and the Coulomb dissociation of ${ }^{8} \mathrm{~B}$. Measurements with these techniques will have the advantage of avoiding some of the systematic uncertainties inherent in the previous $(p, \gamma)$ measurements [e.g., questions relating to target uniformity and the determination of the ${ }^{7} \mathrm{Be}$ content of the target through the ${ }^{7} \operatorname{Li}(d, p)^{8} \mathrm{Li}$ reaction]. At the same time these new techniques will have systematic uncertainties of their own which will need to be understood in order to interpret the results of these new experiments together with the previous $(p, \gamma)$ results. Initial measurements of the Coulomb dissociation of ${ }^{8} \mathrm{~B}$ have already been reported (Motobayashi et al. 1994), indicating that their preliminary results are consistent with the $(p, \gamma)$ results of Filippone, et al. (1983a, 1983b) and are encouraging for further studies to provide an independent determination of $S_{17}(0)$. Recently, the NABONA collaboration has measured initial data utilizing a ${ }^{7} \mathrm{Be}$ beam and a hydrogen gas target (Gialanella et al., 1996).

\section{E. The ${ }^{7} \mathrm{Li}(n, \gamma)^{8} \mathrm{Li}$ reaction}

This reaction is of interest both as the isospin mirror of the ${ }^{7} \mathrm{Be}(p, \gamma)^{8} \mathrm{~B}$ reaction and as the entrance to $A \geqslant 12$ nucleosynthesis in an inhomogeneous big bang. Measurements by Wiescher et al. (1989) had indicated that the cross section for this reaction was a factor of 2 smaller than reported by Imhof et al. (1959), thereby reducing the rate of heavy element synthesis in any primordial $r$ process. However, a number of more recent measurements (Blackmon et al., 1996, and references therein) are in good agreement with the Imhof (1959) results supporting the reaction rate used in the calculation of heavy element production by Malaney and Fowler (1987). It is perhaps interesting to note that the three-cluster generator coordinate method calculations of Descouvemont and Baye (1994), which are consistent with the Imhof/Blackmon ${ }^{7} \mathrm{Li}(n, \gamma){ }^{8} \mathrm{Li}$ results, predict $\mathrm{S}_{17}(0) \geqslant 24 \mathrm{eV}$ b for the isospin mirror, ${ }^{7} \mathrm{Be}(p, \gamma){ }^{8} \mathrm{~B}$ reaction.

\section{F. The ${ }^{14} \mathrm{~N}(p, \gamma){ }^{15} \mathrm{O}$ reaction}

Recent measurements of the ${ }^{14} \mathrm{~N}(p, \gamma){ }^{15} \mathrm{O}$ reaction (Schröder et al., 1987) show clear evidence for interference effects due to the high-energy tail of a subthreshold resonance $\left(E_{\text {c.m. }}=-504 \mathrm{keV} ; J^{\pi}=3 / 2^{+}\right)$, which accounts for nearly half of the total $\mathrm{S}(0)$ for this reaction. As the slowest piece of the $\mathrm{CNO}$ cycle, the rate of this reaction governs the rate of the cycle, and as such plays an important role in determining the evolution of massive 
stars and the ages of globular clusters. Combining the Schröder et al. (1987) measurements with the earlier low-energy $\left(93<E_{\text {c.m. }}<126 \mathrm{keV}\right)$ measurements of Lamb and Hester (1957), determines $S(0)=3.5_{-1.6}^{+0.4} \mathrm{keV} \mathrm{b}$ (INT Workshop, 1997), where the uncertainty arises primarily from uncertainty (because of the interference effects due to the subthreshold resonance) in how to extrapolate the laboratory measurements to zero energy. This uncertainty in the rate of the ${ }^{14} \mathrm{~N}(p, \gamma){ }^{15} \mathrm{O}$ reaction corresponds to an uncertainty of $\sim 1$ Gy in the age of globular clusters (e.g., Chaboyer et al., 1996; and Demarque, 1996).

\section{G. The ${ }^{17} \mathrm{O}(p, \alpha){ }^{14} \mathrm{~N}$ reaction}

The ${ }^{17} \mathrm{O}(p, \alpha){ }^{14} \mathrm{~N}$ reaction provides an important closure for the CNO Tri-Cycle (e.g., Rolfs and Rodney, 1975). Observations of the ${ }^{16} \mathrm{O} /{ }^{17} \mathrm{O}$ ratio in the envelopes of red giants can be combined with information about the production and destruction of ${ }^{17} \mathrm{O}$ in the stellar interior to determine the depth of convective mixing between the surface and the interior of such stars (e.g., Dearborn, 1992). Until recently there was a factor of $\sim 100$ uncertainty/disagreement in the rate of the ${ }^{17} \mathrm{O}(p, \alpha){ }^{14} \mathrm{~N}$ reaction, which is a key to determining the ${ }^{16} \mathrm{O} /{ }^{17} \mathrm{O}$ ratio in stellar nucleosynthesis. For temperatures below $T_{9} \sim 0.1$, the rate of the ${ }^{17} \mathrm{O}(p, \alpha){ }^{14} \mathrm{~N}$ reaction is determined by the properties of the $J^{\pi}=1^{-}$resonance at $E_{\mathrm{c} . \mathrm{m}}=70 \mathrm{keV}$, and a recent direct measurement of the strength of that resonance (Blackmon et al., 1995), $\omega \gamma \approx 5.5 \mathrm{neV}$, has reduced its uncertainty from a factor of $\sim 100$ to $\approx \pm 20 \%$. This much more accurate result will now allow refined observations of the ${ }^{16} \mathrm{O} /{ }^{17} \mathrm{O}$ ratio (particularly in stars with masses greater than 5 solar masses) to place significant constraints on models of convective mixing.

\section{H. The Hot CNO cycle}

One wrinkle, which was not anticipated in $\mathrm{B}^{2} \mathrm{FH}$, was the Hot CNO cycle that can become important when temperatures and densities are sufficiently high so that the ${ }^{13} \mathrm{~N}(p, \gamma){ }^{14} \mathrm{O}$ reaction is fast enough to bypass the normal beta decay of ${ }^{13} \mathrm{~N}$. This possibility was first described by Caughlan and Fowler (1962), and direct measurements of the rate of the ${ }^{13} \mathrm{~N}(p, \gamma){ }^{14} \mathrm{O}$ reaction have recently been made with radioactive beams; using ${ }^{13} \mathrm{~N}$ beams to measure the reaction directly (Decrock et al., 1991), and using ${ }^{14} \mathrm{O}$ beams to measure the inverse reaction, ${ }^{14} \mathrm{O}(\gamma, p){ }^{13} \mathrm{~N}$ (Motobayashi et al., 1991; Kiener et al., 1993). These three experiments are all in good agreement and measure a strength $\omega \gamma=2.3 \pm 0.5 \mathrm{eV}$ for the $E_{r}=529 \mathrm{keV}, \ell=0$, resonance that determines the rate of this reaction at nova temperatures. These results indicate that at the temperatures characteristic of a nova explosion $\left(0.2 \leqslant T_{9} \leqslant 0.4\right)$, the Hot CNO cycle will be the dominant energy generation process. One important consequence of the Hot $\mathrm{CNO}$ cycle is the production of an $\left[{ }^{15} \mathrm{~N} /{ }^{14} \mathrm{~N}\right]$ isotope ratio of $\sim 1$ (compared to the ratio of $\sim 4 \times 10^{-5}$ produced in the CNO cycle), indicating that in the solar system $\left(\left[{ }^{15} \mathrm{~N} /{ }^{14} \mathrm{~N}\right] \approx 4 \times 10^{-3}\right)$ most of the observed ${ }^{15} \mathrm{~N}$ was produced in nova explosions. At still higher temperatures, the reaction sequence ${ }^{15} \mathrm{O}(\alpha, \gamma){ }^{19} \mathrm{Ne}(p, \gamma){ }^{20} \mathrm{Na}$ may provide a breakout path from the Hot CNO Cycle to the $r p$ process (Wallace and Woosley, 1981), which can provide contributions to the nucleosynthesis of proton rich isotopes up through ${ }^{65} \mathrm{As}$ (e.g., Mohar et al., 1991), to as high as ${ }^{68} \mathrm{Se}$ (e.g., Blank et al., 1995), or even to ${ }^{96} \mathrm{Ru}$ (Schatz et al., 1997).

\section{THE $\times$ PROCESS}

\section{A. Introduction and retrospective}

It is quite remarkable how correct most of the basic ideas and the data were that were presented by $\mathrm{B}^{2} \mathrm{FH}$ on the $x$ process. In particular, the solar $\mathrm{Li}$ and $\mathrm{Be}$ abundances determined by Greenstein and Richardson (1951) and Greenstein and Tandberg-Hanssen (1954) are very close to the contemporary values. And whereas the meteoritic Suess-Urey (1957) value for Be is near the the solar value, the solar $\mathrm{Li}$ was found to be 100 times smaller than the meteoritic, still the situation today.

$\mathrm{B}^{2} \mathrm{FH}$ point out that $\mathrm{Li}, \mathrm{Be}$, and $\mathrm{B}$ are extremely rare compared to the elements near them on the periodic table $(\mathrm{H}, \mathrm{He}, \mathrm{C}, \mathrm{N}, \mathrm{O})$, but they are 100 times more abundant than the majority of the heavy elements. $\mathrm{B}^{2} \mathrm{FH}$ give the nuclear reactions that destroy the rare light elements, and that occur at temperatures found in stellar interiors, in the hydrogen-burning zone. These reactions convert the light elements into $\mathrm{He}$ isotopes through fusion with protons. The authors point out that before a star evolves off the main sequence $\mathrm{Li}, \mathrm{Be}$, and $\mathrm{B}$ are destroyed in the vast preponderance of a star's mass. Given this circumstance, a setting other than stellar interiors is required for the synthesis of these light elements. Possibilities such as spallation reactions in stellar atmospheres and supernovae shells were discussed by $\mathrm{B}^{2} \mathrm{FH}$, as was the preservation of ${ }^{7} \mathrm{Li}$ as ${ }^{7} \mathrm{Be}$ in late-type stars.

\section{B. Abundances}

Because $\mathrm{Li}, \mathrm{Be}$, and $\mathrm{B}$ are so readily destroyed in stars, their "cosmic" or "initial" abundances need to be determined in environments that are unaffected by nuclear processing in the interiors. The interstellar gas? The atmospheres of the youngest stars? Primitive solar system material? The first generation of stars?

Atomic and spectroscopic properties of these elements conspire to make all these species difficult to observe. Because the abundances are low, it is best to observe these elements through their absorption in the resonance lines of their various ions. For $\mathrm{Li}$, the resonance doublet of $\mathrm{Li}$ I at $6707 \AA$ is readily observed, but even in the coolest stars, $\mathrm{Li}$ is predominantly ionized because it has a low ionization potential as it is an alkali metal. And Li II is like He I with two electrons in a 
closed shell and has its resonance line at $199 \AA$. The resonance line of $\mathrm{Be} \mathrm{I}$ is at $2348.6 \AA$, unobservable with ground-based telescopes. The resonance doublet of Be II is near $3130 \AA$ where only about $30 \%$ of the flux penetrates the Earth's atmosphere. In solar-type stars, where the dominant ion is Be II, this region of the spectrum is crowded with lines of other elements. For B, both B I and B II resonances lines are in the ultraviolet at 2497.7 and $1362.5 \AA$, respectively. Nonetheless, the importance of these elements-as tracers of stellar structure, galactic evolution, and cosmology, and as part of the picture of the origin of the elements-has led to a huge amount of research work on determining their abundances in many astrophysical environments.

\section{Lithium}

With the exception of the hot $\mathrm{O}$ and $\mathrm{B}$ stars containing no ions of $\mathrm{Li} \mathrm{I}$ in their atmospheres, $\mathrm{Li}$ abundances have been determined for stars of every spectral type and luminosity class. Among the first of a veritable blizzard of papers surveying stellar Li contents was by Herbig (1965). More recent surveys of solar-type field stars include Duncan (1981), Boesgaard and Trippico (1986), Balachandran (1990), and Lambert et al. (1991). The Pop I maximum for field stars is about $\log \mathrm{N}(\mathrm{Li})=3.1-$ 3.3. There have been several studies on $\mathrm{Li}$ abundances in the youngest stars, $\mathrm{T}$ Tauri stars, which include Zapala (1972), Padgett (1990, 1991), Basri et al. (1991), King (1993), Lee et al. (1994), Cunha et al. (1995), and Duncan and Rebull (1996). Cunha et al. (1995) find a mean Li abundance for stars in the Orion association, as corrected for nonlocal thermodynamic equilibrium effects (see below), to be $\log \mathrm{N}(\mathrm{Li})=3.2( \pm 0.1)$ which is very close to the meteoritic value of 3.3 and conclude that there has been little Li enrichment in the Galaxy in the last 5 Gyr.

There has been considerable work done on $\mathrm{Li}$ abundances in open clusters, partly with the goal of obtaining an "initial" Li abundance for Pop I stars. See Thorburn et al. (1993 and references therein) for a partial review of the subject. The maximum $\mathrm{Li}$ abundances for stars in a given cluster can be found in the temperature region near $6200 \mathrm{~K}$ with the youngest clusters having the most Li (Boesgaard, 1991). Deliyannis et al. (1994) show that short-period tidally locked binaries (SPTLB) near that temperature in the Pleiades, age $\sim 80 \mathrm{Myr}$, have $\mathrm{Li}$ abundances on the mean trend, while in the Hyades (age $\sim 700 \mathrm{Myr}$ ) and M 67 (age 5 Gyr) they have somewhat higher $\mathrm{Li}$ abundances than their respective clustermates. This suggests that Li depletion has been inhibited in the SPTLB by the lack of rotationally induced mixing and indicates that the Li-age relation of Boesgaard (1991) results from a slow mixing process such as rotation. The maximum abundances for clusters are measured or inferred to be $\log \mathrm{N}(\mathrm{Li})=3.1-3.3$.

It is well known that evolved stars dilute their surface Li content as their convective zones deepen (Iben, 1965, 1967a). More recently Deliyannis, Demarque, and Kawaler (1990) have made dilution calculations for metal-poor stars. Although there has been much observational research on $\mathrm{Li}$ in such stars, it is not relevant to the "initial" Li content and will not be discussed here.

The abundance of $\mathrm{Li}$ in the oldest stars may reveal the primordial (big bang) value of $\mathrm{Li}$. In a study of $\mathrm{Al}$ in halo dwarfs, Spite and Spite (1982a, 1982b) discovered a Li line present in their spectra and found an approximately uniform value for the $\mathrm{Li}$ abundance that was about a factor of 10 lower than the Pop I star maximum. Several surveys since then have confirmed and extended these results: Spite et al. (1984), Rebolo et al. (1988), Hobbs and Duncan (1987), Spite and Spite (1993), Thorburn (1994), and Molaro et al. (1995). Several papers address the constancy of the Li abundance in the lowmetallicity Li plateau, for example Deliyannis et al. (1993), Ryan et al. (1996), Spite et al. (1996). These papers reach different conclusions about the amount of the intrinsic spread in $\mathrm{Li}$ and its dependence on effective temperature and $[\mathrm{Fe} / \mathrm{H}]$. But, as the rotational models predict (Deliyannis, 1990; Pinsonneault et al., 1992; Chaboyer and Demarque, 1994), even a small dispersion could be the result of Li depletion by an order of magnitude. The most comprehensive review of all the data, put on a uniform temperature scale, is by Ryan et al. (1996). They find that the correlation between Li abundance and temperature and $\mathrm{Li}$ abundance and $[\mathrm{Fe} / \mathrm{H}]$ is real. They point out three turn-off stars that have similar temperatures and very low metallicities (between -3.0 and -3.5 in $[\mathrm{Fe} / \mathrm{H}]$ ) for which $3-5 \mathrm{Li}$ observations have been made. The Li abundances are $1.89 \pm 0.05,2.01$ \pm 0.04 , and $2.29 \pm 0.05$, indicative of an intrinsic spread (of a factor of 2.5) due to influences other than the uniform nucleosynthesis of $\mathrm{Li}$ in the Big Bang.

The halo star, BD +233910 , has a $\mathrm{Li}$ abundance that is a factor of 2 to 3 above the plateau (King, Deliyannis, Hiltgen, et al., 1997). This high $\mathrm{Li}$ may result from a lower than average depletion in Li from a higher (primordial) $\mathrm{Li}$ value or may result from some type of galactic Li enrichment mechanism. These possibilities are explored in a search of other spectroscopic signatures of enrichment, but since none of the predicted anomalies are found, it seems more likely that $\mathrm{Li}$ in this star has been preserved from a high initial value.

Recently, Li has been detected in stars near the main sequence turn-off in globular clusters-in NGC 6397 by Molaro and Pasquini (1994) and Pasquini and Molaro (1996) and in M 92 by Deliyannis et al. (1995) and Boesgaard et al. (1997). For NGC 6397 the three turn-off stars have $\log \mathrm{N}(\mathrm{Li})=2.28 \pm 0.10$, higher than the halo field stars. From the Keck study of seven similar M 92 stars there is clear evidence for a dispersion in $\mathrm{Li}$ abundance of 0.5 dex. The $\mathrm{Li}$ abundance in one of the M 92 stars is nearly as high as that in BD +23 3910. The M 92 results were attributed to differences in the amount of $\mathrm{Li}$ depletion in otherwise identical (age, temperature, mass, luminosity, metallicity) stars. The differing degrees of depletion could result from differences in the initial angular momentum of the individual stars and the spindown rates. When the M $92 \mathrm{Li}$ dispersion is coupled with a possible dispersion in $\mathrm{Li}$ in the field star plateau, it 
indicates that there may be some Li depletion there too. The interesting discovery of ${ }^{6} \mathrm{Li}$ in a halo star (Smith et al., 1993) further complicates the interpretation that halo stars contain pristine Big Bang ${ }^{7} \mathrm{Li}$ only. However, see Lambert (1995) and Deliyannis and Malaney (1995) for discussions of stellar self-synthesis of ${ }^{6} \mathrm{Li}$.

Boesgaard and Tripicco (1986) examined their sample of Pop I and old disk stars for a relation between Li and $[\mathrm{Fe} / \mathrm{H}]$. They found the full range of $\mathrm{Li}$ abundances at solar metallicity, but an absence of low metalicity, Lirich stars. Surveys of Rebolo et al. (1988), Balachandran (1990), and Lambert et al. (1991) extend that result and include the Li abundances of the Pop II stars. There is an upper envelope to the $\mathrm{Li}$ abundance with a decrease in $\mathrm{Li}$ abundance as $[\mathrm{Fe} / \mathrm{H}]$ goes from -0.4 to -1.4 . Interpretations differ: if there is an age-metallicity relation and thus a Li-age relation, the upper envelope shows the increase in Li content over the evolution of the galactic disk, while the same relation with age could mean that the older stars have had longer to deplete Li by various "slow" processes from a higher or even much higher $\mathrm{Li}$ abundance.

The abundance of $\mathrm{Li}$ in the sun, $\log \mathrm{N}(\mathrm{Li})=1.16$ (Steenbock and Holweger, 1984), is depleted relative to other Pop I dwarfs and relative to meteorites at 3.3 \pm 0.04 (Anders and Greveese, 1989). King, Deliyannis, Hiltgen, et al. (1997) derive a solar Li abundance of 1.05 from three separate sets of high-resolution, high signalto-noise spectra of the sky and/or the moon. The Li content and isotope ratio has been determined in the interstellar medium, most recently by Lemoine et al. (1995). They conclude that there must be an extra source of ${ }^{7} \mathrm{Li}$ in the Galaxy.

It is likely that both stellar processing of $\mathrm{Li}$, which depletes $\mathrm{Li}$ in the oldest stars, and galactic processing of $\mathrm{Li}$, which contributes to the increased amount of $\mathrm{Li}$ in the youngest stars, exist together. These processes make it difficult to assess the true value for primordial $\mathrm{Li}$. However, since all the stellar processes are known to deplete $\mathrm{Li}$, the Pop II star Li probably sets a lower limit to the primordial $\mathrm{Li}$. The best estimates of that cover the range from $\log \mathrm{N}(\mathrm{Li})=2.2$ to 2.6 .

\section{Beryllium}

The study of Be abundances in Pop I began in the 1960's and was reviewed by Wallerstein and Conti (1969). A survey of Be in solar-type stars was done by Boesgaard (1976) who found that while most such stars had normal (i.e., solar) Be, some of the hotter ones were deficient in Be. Boesgaard and King (1993) presented $\mathrm{Be}$ results for Li-normal, solar-metallicity stars and found a range in abundances of a factor of about three. A survey of Be in some 60 Li-deficient F and G dwarfs (Pop I) by Stephens et al. (1997) shows Be deficiencies in the hotter stars, analogous to the Li dip at those temperatures, and near solar Be abundances for stars cooler than $6000 \mathrm{~K}$. Stars in which both $\mathrm{Li}$ and Be are depleted but present in detectable amounts show a depletion pat- tern very close to the predictions of models by Pinsonneault, Kawalar, and Demarque (1990) of rotationally induced mixing.

There have been several recent studies of $\mathrm{Be}$ in halo stars: Gilmore, Edvardsson, and Nissen (1991), Gilmore et al. (1992), Rebolo et al. (1988), Ryan et al. (1992), Boesgaard and King (1993), Garcia-Lopez et al. (1995), a Keck Be project reported by Boesgaard (1996), Thorburn and Hobbs (1996), Primas (1996), and Deliyannis et al. (1997). All show an increase in Be in stars as the metallicity increases. The slope of $\log \mathrm{N}(\mathrm{Be})$ vs $[\mathrm{Fe} / \mathrm{H}]$ is close to 1.0. There is no evidence of a Be plateau at the lowest $[\mathrm{Fe} / \mathrm{H}]$ values; the lowest $\mathrm{Be}$ detection is $\log$ $\mathrm{N}(\mathrm{Be}) / \mathrm{N}(\mathrm{H})=-13.5$ for $\mathrm{BD}-133442$ at $[\mathrm{Fe} / \mathrm{H}]=-3.0$. This evidence points to a galactic-wide production mechanism and no signature of any primordial $\mathrm{Be}$.

The solar $\mathrm{Be} / \mathrm{H}$ value has been well determined by Chmielewski, Muller, and Brault (1975) as $\mathrm{Be} / \mathrm{H}=1.4$ $( \pm 0.6) \times 10^{-11}$. The meteoritic value given by Anders and Grevesse (1989) is $2.6 \times 10^{-11}$. Recent work by King, Deliyannis, and Boesgaard (1997), who include the solar Be spectrum in their study of $\mathrm{Be}$ in $\alpha$ Cen $\mathrm{A}$ and $\mathrm{B}$, also indicates that the sun is depleted in Be relative to meteorites by a factor of 1.4 to 3.2. The sun appears to be mildly depleted in $\mathrm{Be}$ while still retaining some $\mathrm{Li}$, which suggests a slow-mixing process below the surface convection zone.

\section{Boron}

Under the observational evidence section on the $x$ process, $\mathrm{B}^{2} \mathrm{FH}$ say only "Boron is spectroscopically unobservable." In fact the resonance lines of both B I and B II are in the ultraviolet region of the spectrum, unobservable from the ground. However, with the advent of the Copernicus satellite in 1972, the IUE in 1978, and the Hubble Space telescope (HST) in 1990, B has finally been seen.

Vega was discovered to have the resonance line of $\mathrm{B}$ II at $1362 \AA$ by Boesgaard et al. (1974) with Copernicus observations at $0.2 \AA$ resolution. The $\mathrm{B} / \mathrm{H}$ abundance derived, with an estimate made for departures from local thermodynamic equilibrium, was $1.0 \times 10^{-10}$. Additional observations by Praderie et al. (1977) confirmed this result from spectra with $0.05 \AA$ resolution. In a study of 16 normal A and B stars, Boesgaard and Heacox (1978) made Copernicus observations to determine $\mathrm{B} / \mathrm{H}$ abundances from the B II resonance line. After correction for non-LTE effects, they give a mean value for cosmic $\mathrm{B} / \mathrm{H}$ of $2( \pm 0.9) \times 10^{-10}$.

Recent results for B in stars come from HST observations. Examples of papers on B abundances for Pop I stars are Lemke et al. (1993), Duncan et al. (1994), Cunha et al. (1997), and for Pop II stars are Duncan et al. (1992), Edvardsson et al. (1994), Duncan et al. (1997). The papers on Pop II stars probe the relationship between the $\mathrm{B}$ abundance and the $\mathrm{Fe}$ abundance and assess the $\mathrm{B} / \mathrm{Be}$ ratio. The slope of the relation between $\log \mathrm{N}\left(\mathrm{B}_{L T E}\right)$ and $[\mathrm{Fe} / \mathrm{H}]$ is $\sim 1$ and between $\log$ 
$\mathrm{N}\left(\mathrm{B}_{N L T E}\right)$ and $[\mathrm{Fe} / \mathrm{H}]$ is $\sim 0.7$ and the $\mathrm{B} / \mathrm{Be}$ ratio is typically $\sim 15$ (Duncan et al., 1997).

The solar abundance of B has been determined from the resonance line of B I at $\lambda 2496.8$ by Kohl, Parkinson, and Withbroe (1976) with a rocket-borne photoelectric detector with a spectral resolution of $0.028 \AA$; they derived a $\mathrm{B} / \mathrm{H}$ abundance of $1.6 \times 10^{-10}$. Zhai and Shaw (1994) find meteorites to have $16.9 \pm 2.2 \mathrm{~B}$ atoms per $10^{6}$ $\mathrm{Si}$ atoms or $\mathrm{B} / \mathrm{H}=6.0 \times 10^{-10}$. Anders and Grevesse (1989) report the ${ }^{11} \mathrm{~B} /{ }^{10} \mathrm{~B}$ to be 4.05 . Boron has been found in the interstellar gas from HST observations, e.g., Federman et al. (1993), Jura et al. (1996), with the abundance of $\mathrm{B} / \mathrm{H}$ of $0.9 \times 10^{-10}$. Federman et al. (1996) find the isotope ratio, ${ }^{11} \mathrm{~B} /{ }^{10} \mathrm{~B}$, in the interstellar medium to be $3.4_{-0.6}^{+1.3}$, probably not different from the meteoritic value.

\section{Nonlocal thermodynamic-equilibrium effects}

The above analyses were based on the assumption of local thermodynamic equilibrium. At the time of the publication of the $\mathrm{B}^{2} \mathrm{FH}$ paper, the actual solutions for the corrections needed were years in the future. For $\mathrm{Li}$ the most complete results are presented by Carlsson et al. (1994). For B similar results can be found in Kiselman (1994) and Kiselman and Carlsson (1996). An interesting situation prevails for Be: neutral Be atoms suffer overionization and the ground state of Be II is over populated, but bound-bound pumping overexcites Be II which depopulates the ground state. For solar-type stars the overionization is roughly balanced by the overexcitation and the effects of NLTE, which exist, cancel each other (Kiselman and Carlsson, 1995; Garcia-Lopez et al., 1995).

\section{Production mechanisms}

\section{Big Bang}

Big Bang nucleosythesis of light isotopes has been predicted by "standard" models by Wagoner et al. (1967), Wagoner (1973), and Thomas et al. (1993) and by inhomogeneous models by Thomas et al. (1994). Only the isotope ${ }^{7} \mathrm{Li}$ can be produced in substantial quantities in the standard models. At low values of the nucleon to photon ratios (or baryonic mass densities) it is produced mainly by ${ }^{4} \mathrm{He}\left({ }^{3} \mathrm{H}, \gamma\right){ }^{7} \mathrm{Li}$. As the ratio increases, that ${ }^{7} \mathrm{Li}$ is destroyed by ${ }^{7} \mathrm{Li}(p, \alpha){ }^{4} \mathrm{He}$ reactions. At still higher ratios, ${ }^{7} \mathrm{Li}$ can be produced by ${ }^{4} \mathrm{He}\left({ }^{3} \mathrm{He}, \gamma\right){ }^{7} \mathrm{Be}\left(e^{-}, \nu_{e}\right){ }^{7} \mathrm{Li}$. The two processes result in a dip for the $\mathrm{Li}$ abundance at intermediate values of the nucleon to photon ratio $\left(1-7 \times 10^{-10}\right)$. Predicted ${ }^{7} \mathrm{Li} / \mathrm{H}$ ratios cover the range of $10^{-10}$ to $10^{-9}$. See Boesgaard and Steigman (1985) and Krauss and Romanelli (1990) for more discussion of the synthesis of ${ }^{7} \mathrm{Li}$ during the Big Bang.

\section{Spallation}

There is the suggestion in $\mathrm{B}^{2} \mathrm{FH}$ that $\mathrm{Li}, \mathrm{Be}$, and $\mathrm{B}$ could be produced by spallation reactions on abundant elements such as $\mathrm{C}, \mathrm{N}, \mathrm{O}, \mathrm{Fe}$ with energies $>100 \mathrm{MeV}$ per nucleon. Although the site was thought to be stellar atmospheres, it was shown by Ryter et al. (1970) that production of light elements in the atmospheres of $\mathrm{T}$ Tauri stars was energetically unfeasible. Reeves et al. (1970) suggested that energetic galactic cosmic rays could bombard $\mathrm{C}, \mathrm{N}, \mathrm{O}$ atoms, breaking them up into the light isotopes. This concept was expanded by Meneguzzi et al. (1971). Their model was successful in many aspects, but it underproduced ${ }^{7} \mathrm{Li}$ and could not match the observed isotope ratios for $\mathrm{Li}$ and $\mathrm{B}$. A low-energy "carrot" of cosmic ray flux was added to help these problems. However, some additional ${ }^{7} \mathrm{Li}$ is produced in the Big Bang and in the envelopes of AGB stars. An interesting addition to spallation production is that spallation in the halo would be more efficient than $\alpha+\alpha$ reactions (Steigman and Walker, 1992).

In addition to accounting for the present day light element ratios, spallation models can be constrained better if they are required to explain the evolution of light elements over the history of the Galaxy. Information has become available on the evolution of both $\mathrm{Be}$ and $\mathrm{B}$ (as revealed by the use of $[\mathrm{Fe} / \mathrm{H}]$ as a chronometer) and the apparent constancy of Li. Models of galactic chemical evolution have been made to interpret the evolution of the light elements (Vangioni-Flam et al., 1990; Ryan et al., 1992; Prantzos, Cassé, and Vangioni-Flam, 1993; Feltzing and Gustafsson, 1994; Tayler, 1995; Lemoine et al., 1997). Duncan et al. (1997) interpret the most recent observations of $\mathrm{B}$ and $\mathrm{Be}$ evolution to favor a somewhat different version of the classical spallation production: it is the $\mathrm{C}$ and $\mathrm{O}$ atoms that bombard the ambient protons and alpha-particles. Vangioni-Flam et al. (1996) show that spallation by energetic $\mathrm{C}$ and $\mathrm{O}$ atoms can account for the nearly constant increase in $[\mathrm{Be}]$ and $[\mathrm{B}]$ with $[\mathrm{Fe} / \mathrm{H}]$. The source of $\mathrm{C}$ and $\mathrm{O}$ is from winds from massive stars, such as Wolf-Rayet stars, and massive supernovae. However, Prantzos et al. (1993) and Malaney and Butler (1993) argue that this mechanism has serious problems.

\section{Asymptotic giant branch stars}

It was pointed out in $\mathrm{B}^{2} \mathrm{FH}$ that Li-rich red giants had been discovered and that Cameron (1955) had proposed preserving ${ }^{7} \mathrm{Li}$ in the form of ${ }^{7} \mathrm{Be}$ in the hydrogenburning region. This mechanism was rediscussed by Cameron and Fowler (1971). There is very interesting modern data to support the production of ${ }^{7} \mathrm{Li}$ in AGB stars, first presented by Smith and Lambert $(1989,1990)$ for AGB stars in the Magellanic clouds. This work has been extended by Plez et al. (1993) and by Smith et al. (1995). The stars in the Magellanic clouds with Li excesses of 100-1000 times over the predicted (diluted) values range between bolometric magnitudes $M_{\mathrm{bol}}=-7.2$ to -6.5 corresponding to 4-8 $M_{\odot}$. Predictions have been made by Scalo et al. (1975) and Sackmann and Boothroyd (1992) that hot-bottom convective envelope burning would produce and then transport ${ }^{7} \mathrm{Be}$ to the observable surface of AGB stars where it becomes ${ }^{7} \mathrm{Li}$ 
through electron capture. The predictions and the observations are in good agreement. This mechanism may produce a substantial amount of enrichment in the galactic $\mathrm{Li}$ abundance over the Big Bang production (unless, of course, it is the case that Big Bang ${ }^{7} \mathrm{Li}$ was as high as the Pop I star value of 3.1-3.3). In any event, it is probable that this production mechanism exists inasmuch as there are some very Li-rich late-type giants, particularly carbon stars, many of which had been discovered by the time of the $\mathrm{B}^{2} \mathrm{FH}$ review and are referenced there.

\section{Supernovae}

In 1973 Audouze and Truran suggested that Li and B could be produced in supernovae shells from high energy particles that were produced in the shock waves; this was another component of spallation. However, today's theories for supernovae production of the light elements are very different. Models of type II supernovae by Woolsey et al. (1990) and Timmes et al. (1995) suggest a " $\nu$ process" that produces B and $\mathrm{Li}$ in supernovae envelopes. As a massive star undergoes core collapse to form a neutron star, it generates a huge flux of neutrinos. Although the cross sections for collision are small there are so many neutrinos that they excite heavy elements. Since deexcitation by particle emission is almost always favored over radiative deexcitation, the affected nuclei are likely to emit a proton, neutron or alpha particle. Among the products created are ${ }^{11} \mathrm{~B}$ and ${ }^{7} \mathrm{Li}$.

There is some evidence that indicates that this mechanism does not play a dominant role. Duncan et al. (1997) conclude that spallation by low energy $\mathrm{C}$ and $\mathrm{O}$ atoms onto protons and $\alpha$ particles in the vicinity of massive supernovae produce sufficient $\mathrm{B}$ and $\mathrm{Be}$ in the early history of the Galaxy and that the $\nu$ process would produce only a small part of the total B. Further evidence that the $\nu$ process would play only a minor role comes from Cunha et al. (1997) who found that two of their four stars in the Orion Association are $\mathrm{O}$ rich (by $60 \%$ ) and $\mathrm{B}$ deficient (by $250 \%$ ). If supernova ejecta produce the increase in $\mathrm{O}$, there should be an increase in $\mathrm{B}$, not the decrease observed.

\section{HELIUM BURNING}

As hydrogen burns in a star, a hot, dense core of helium is formed that fuels the nucleosynthesis of the heavier elements. The first stage of this process is the so-called "triple- $\alpha$ " capture to form ${ }^{12} \mathrm{C}$, followed by the subsequent capture of $\alpha$ particles to form ${ }^{16} \mathrm{O}$. In essence, helium burning terminates there, because further $\alpha$ captures (to form ${ }^{20} \mathrm{Ne}$, for example) occur too slowly at these temperatures and densities to be significant.

The termination of helium burning at ${ }^{16} \mathrm{O}$ was not realized at the time of $\mathrm{B}^{2} \mathrm{FH}$, because of uncertainty in the level structure of ${ }^{20} \mathrm{Ne}$ near the $\alpha+{ }^{16} \mathrm{O}$ threshold. However, it was later discovered that the levels closest to the threshold had the wrong parity and/or angular momentum to make a large resonant contribution to the

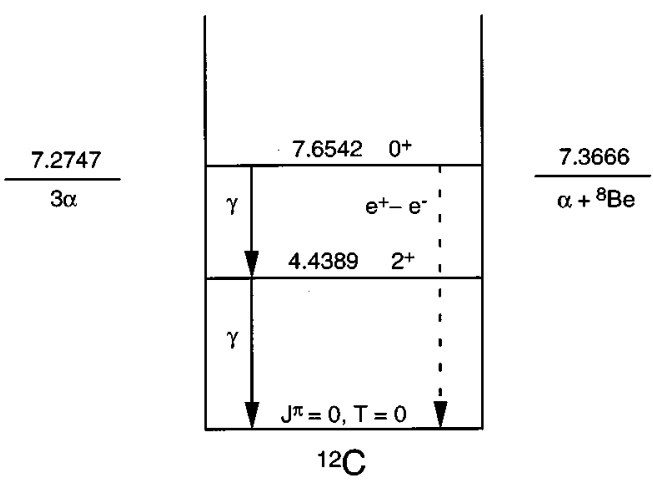

FIG. 2. The astrophysically important energy levels of ${ }^{12} \mathrm{C}$.

rate. A calculation by Fowler et al. (1975) showed that the rate for ${ }^{16} \mathrm{O}(\alpha, \gamma){ }^{20} \mathrm{Ne}$ is far below that for ${ }^{12} \mathrm{C}(\alpha, \gamma){ }^{16} \mathrm{O}$ for $\mathrm{T}_{9} \leqslant 0.2$. Therefore, the blocking of further $\alpha$ captures for normal helium-burning conditions allows us to concentrate on triple- $\alpha$ and $\alpha+{ }^{12} \mathrm{C}$ capture in the following discussion. Although the formulas used to describe the rates of these reactions are essentially unchanged from forty years ago, the major advance, as already pointed out earlier, has been the experimental determination, with great precision in some cases, of the nuclear parameters upon which the expressions depend.

\section{A. Triple- $\alpha$ capture}

This reaction actually occurs in two stages: first, two $\alpha$ particles resonate in the low-lying (but unbound) state that forms the ground state of ${ }^{8} \mathrm{Be}$. This state is sufficiently long-lived $\left(\tau_{1 / 2}=0.968 \times 10^{-16} \mathrm{~s}\right)$ that there is a non-neglible probability that a third $\alpha$ particle will be captured before it disintegrates, forming ${ }^{12} \mathrm{C}^{* *}\left(E_{x}=7.6542 \mathrm{MeV}, J^{\pi}=0^{+}\right)$. A level diagram showing the relevant states and thresholds for ${ }^{12} \mathrm{C}$ is given in Fig. 2. Because of its quantum numbers, there is only a small probability that this excited state will deexcite (rather than decay back into three $\alpha$ particles), either by $e^{+}-e^{-}$pair production, or by a $\gamma$ ray cascade through the first excited state, leaving ${ }^{12} \mathrm{C}$ in its $0^{+}$ ground state. The prediction (Hoyle et al., 1953), and subsequent experimental verification, of the properties of ${ }^{12} \mathrm{C}^{* *}$ in order to account for the observed abundance of ${ }^{12} \mathrm{C}$ remains one of the most impressive accomplishments of nuclear astrophysics.

The rate per unit volume for the deexcitation process at temperature $T$ is given by the resonance form (Rolfs and Rodney, 1988)

$$
r_{3 \alpha}=\frac{N_{\alpha}^{3}}{2} 3^{3 / 2}\left(\frac{2 \pi \hbar^{2}}{M_{\alpha} k T}\right)^{3} \frac{\Gamma_{\alpha} \Gamma_{\mathrm{rad}}}{\hbar \Gamma} \exp \left(-\frac{Q}{k T}\right),
$$

with $N_{\alpha}$ the $\alpha$ particle number density, $M_{\alpha}$ its mass,

$$
Q=\left(M^{12} \mathrm{C}_{* *}-3 M_{\alpha}\right) c^{2}=379.5 \pm 0.3 \mathrm{keV},
$$

and $\Gamma_{\alpha}$ and $\Gamma_{\text {rad }}$ the decay widths for $\alpha$ and $\left(e^{ \pm}+\gamma\right)$ emission, respectively, which sum to the total width $\Gamma$. Since $\Gamma_{\text {rad }} \ll \Gamma_{\alpha} \approx \Gamma$, the triple- $\alpha$ rate depends only on $\Gamma_{\text {rad }}=\Gamma_{\gamma}+\Gamma_{\text {pair }}$. The current values (Ajzenberg- 
Selove, 1990) of these widths are $\Gamma_{\gamma}=(3.64 \pm 0.50) \mathrm{meV}$ and $\Gamma_{\text {pair }}=(60.5 \pm 3.9) \mu \mathrm{eV}$. The underestimation of this rate by $\mathrm{B}^{2} \mathrm{FH}$ due to their value of $\Gamma_{\gamma}=1 \mathrm{meV}$ was compensated in part by having at that time a smaller $Q$ value $(372 \mathrm{keV})$ in the exponential factor of Eq. (8).

\section{B. $\alpha+{ }^{12} \mathrm{C}$ capture}

The rate of this reaction is responsible for one of the most important uncertainties in nuclear astrophysics today. Its rate at stellar temperatures, relative to that of "triple- $\alpha$ " capture, determines how much of the ${ }^{12} \mathrm{C}$ formed is converted to ${ }^{16} \mathrm{O}$, and thereby the carbon/ oxygen abundance ratio in the ejecta of massive stars and in the interiors of white dwarfs formed by stars initially less massive than $\sim 11 M_{\odot}$. The relative amounts of carbon and oxygen at the end of the helium-burning phase set the initial conditions for the next phase in massive stars, which is heavy-ion burning. In the process, many of the heavier elements up through iron are synthesized. All of these processes have been found to be quite sensitive to the $\alpha+{ }^{12} \mathrm{C}$ capture rate (Weaver and Woosley, 1993), so that the abundances of the mediummass elements, and even the final evolution of massive stars that explode as supernovae, depend critically on its determination.

The rate per unit volume for $\alpha+{ }^{12} \mathrm{C}$ capture is given by the familiar expression involving the number densities $N_{\alpha}$ and $N^{12} \mathrm{C}$, and the Maxwellian-averaged $\langle\sigma v\rangle$ for temperature $T$,

$$
r_{\alpha+12 \mathrm{C}}=N_{\alpha} N_{12} \mathrm{C}\left\langle\sigma_{\mathrm{cap}} v\right\rangle_{T} .
$$

The cross section is parametrized as

$$
\sigma_{\text {cap }}(E)=\frac{S_{\text {cap }}(E)}{E} \exp (-2 \pi \eta)
$$

in terms of Sommerfeld's Coulomb parameter $\eta$ for $\alpha+{ }^{12} \mathrm{C}$, and the $S$ factor for the capture reaction,

$$
S_{\text {cap }}(E)=S_{E 1}(E)+S_{E 2}(E),
$$

where $\eta=Z_{1} Z_{2} e^{2} / \hbar v$.

The $\langle\sigma v\rangle$ integral is mainly determined by the value of the cross section (or $S$ factor) at the Gamow energy, which for helium-burning temperatures $\left(\mathrm{T}_{9} \approx 0.2-0.6\right)$ is $E_{0}=0.3-0.9 \mathrm{MeV}$. Since present measurements cannot be extended to such low energies in the presence of Coulomb barriers as large as that for $\alpha+{ }^{12} \mathrm{C}$, the rate must be found by theoretical extrapolation. Due to their different energy dependencies, the $E 1$ and $E 2$ multipole components of the cross section are extrapolated separately to $E=E_{0}$, both being influenced by the presence of subthreshold levels in ${ }^{16} \mathrm{O}$ having $J^{\pi}=1^{-}$and $2^{+}$, respectively. The levels of ${ }^{16} \mathrm{O}$ near the $\alpha+{ }^{12} \mathrm{C}$ threshold are shown in Fig. 3.

\section{E1 capture}

Much effort has been devoted to extracting just the $E 1$ part of the capture cross section, which is dominated by a broad $1^{-}$resonance at $E=2.4 \mathrm{MeV}\left(E_{x}=9.585\right.$

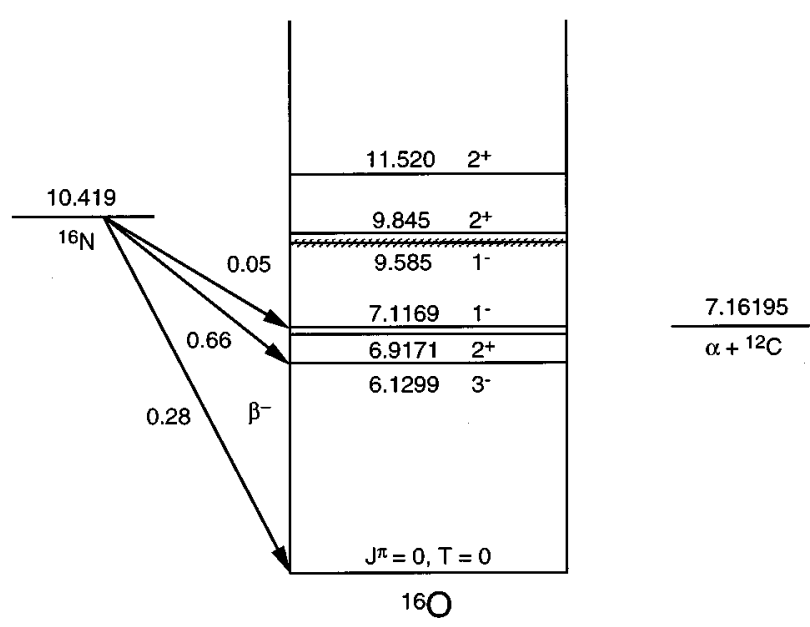

FIG. 3. The astrophysically important energy levels of ${ }^{16} \mathrm{O}$.

$\mathrm{MeV}$; all level energies and widths for ${ }^{16} \mathrm{O}$ are taken from Tilley et al., 1993). The importance of the subthreshold $1^{-}$state at $E=-45 \mathrm{keV}\left(E_{x}=7.11685 \mathrm{MeV}\right)$ in fitting and extrapolating the $E 1$ cross section was first demonstrated by Dyer and Barnes (1974). Most subsequent analyses have fit $S$ factors extracted from the measurements along with the $P$ wave phase shift obtained from $\alpha+{ }^{12} \mathrm{C}$ elastic scattering measurements (Plaga et al., 1987), using either $R$-matrix or $K$-matrix theory. More recently, several groups have acted on the longstanding suggestion of Barker (1971) to measure the $\beta$-delayed $\alpha$ spectrum from the decay of ${ }^{16} \mathrm{~N}$ with the hope of better determining the contribution of the subthreshold state, which is evident in the spectrum as a secondary maximum at low energies.

\section{a. Direct measurements}

Several direct measurements (Dyer and Barnes, 1974; Redder et al., 1987; Kremer et al., 1988; Ouellet et al., $1992)$ of the $E 1$ capture cross section have been made in the c.m. energy range $E=1.0-3.0 \mathrm{MeV}$. With the recently reported corrections in the data of Ouellet et al. (1996), these measurements are in relatively good agreement at energies between 1.3 and $3.0 \mathrm{MeV}$. They now all imply constructive interference of the subthreshold level with the positive energy resonance [originally, Ouellet et al. (1992), had found destructive interference], leading to extrapolated $S$ factors at $E=0.3 \mathrm{MeV}$ that range from 10 to $200 \mathrm{keV} \mathrm{b}$. The extrapolated $S$ factors obtained from these measurements, along with their assigned uncertainties, are given in Table II. The table also notes briefly the methods used to make and analyze the measurements. Most of the analyses involved doing standard $R$-matrix or $K$-matrix fitting, or using the "hybrid" $R$-matrix method (Koonin, Tombrello, and Fox, 1974), in which a potential is used to represent the resonances at $2.4 \mathrm{MeV}$ and above, in order to reduce the uncertainty in the "background" contribution to the $R$ matrix. 
TABLE II. Extrapolated values of $S_{E 1}\left(E_{0}=0.3 \mathrm{MeV}\right)$ obtained from direct measurements of the differential capture cross section.

\begin{tabular}{|c|c|c|}
\hline Reference & $S_{E 1}\left(E_{0}\right)(\mathrm{keV} \mathrm{b})$ & Methods \\
\hline \multirow[t]{3}{*}{ Dyer and Barnes (1974) } & & $\begin{array}{l}\text { measured } \sigma\left(90^{\circ}\right) ; \text { corrected for } E 2 \text { with } \\
\text { direct-capture calculations }\end{array}$ \\
\hline & $140_{-120}^{+140}$ & 3-level $R$ matrix \\
\hline & $80_{-40}^{+50}$ & hybrid $R$ matrix \\
\hline \multirow[t]{3}{*}{ Redder et al. (1987) } & & measured $\sigma(\theta)$; separated $E 1$ and $E 2$ \\
\hline & $200_{-110}^{+270}$ & 3-level $R$ matrix \\
\hline & $140_{-80}^{+120}$ & hybrid $R$ matrix \\
\hline \multirow[t]{2}{*}{ Kremer et al. (1988) } & & measured $\sigma\left(90^{\circ}\right)$, using $\gamma$-recoil coincidence \\
\hline & $10_{-10}^{+130}$ & $\begin{array}{l}\text { 3-level } R \text { matrix, constrained by } l=1 \alpha+{ }^{12} \mathrm{C} \\
\text { phase shift }\end{array}$ \\
\hline \multirow[t]{2}{*}{ Ouellet et al. (1996) } & & measured $\sigma(\theta) ;$ separated $E 1$ and $E 2$ \\
\hline & $79 \pm 16$ & $\begin{array}{l}R \text {-matrix, } K \text {-matrix fits; includes } \beta \text {-delayed } \alpha \\
\text { spectra }\end{array}$ \\
\hline
\end{tabular}

\section{b. $\beta$-delayed $\alpha$ spectrum from the decay of ${ }^{16} \mathrm{~N}$}

At least three groups (Buchmann et al., 1993; Zhao et al., 1993; Zhao et al., 1995) have measured the delayed $\alpha$ spectrum from the $\beta$ decay of ${ }^{16} N$ down to energies low enough to see the secondary maximum attributed to the presence of the subthreshold $1^{-}$state. Some of these new spectral measurements have been included in the fitting along with direct measurements of the $E 1$ cross section, in order to better constrain the parameters (in particlular, the reduced $\alpha$ width) of the subthreshold state. These constrained analyses give extrapolated values for $S_{E 1}\left(E_{0}\right)$ in the range $80-95 \mathrm{keV} \mathrm{b}$, as favored by the sensitivity study (Weaver and Woosley, 1993) of the dependence of calculated elemental abundances on the extrapolated $\alpha+{ }^{12} \mathrm{C}$ capture cross section.

The functional form of the spectrum can be obtained from the usual $R$-matrix relation of the scattering states to the level matrix, giving

$$
\frac{d N_{\alpha}}{d E}=f_{\beta}(E) P_{\alpha}(E)\left|\sum_{\lambda} B_{\lambda} \sum_{\lambda^{\prime}} A_{\lambda \lambda^{\prime}}(E) \gamma_{\lambda^{\prime} \alpha}\right|^{2},
$$

in which $f_{\beta}$ is Fermi's integrated $\beta$ decay function for $Z=8, P_{\alpha}$ is the $\alpha+{ }^{12} \mathrm{C}$ penetrability, $B_{\lambda}$ is the dimensionless $\beta$-feeding amplitude for level $\lambda, A$ is the level matrix, and $\gamma_{\lambda^{\prime} \alpha}$ is the reduced-width amplitude in the $\alpha+{ }^{12} \mathrm{C}$ channel for level $\lambda^{\prime}$. The product of the first two terms in the expression gives the phase-space behavior of the spectrum, while the third term gives the structure. A similar expression results from the $K$-matrix formalism (Humblet, Filippone, and Koonin, 1991).

The interference minimum between the two maxima in the spectrum cannot fix the interference of the levels in the cross section, due to the undetermined relative phases of the $B_{\lambda}$. However, as was mentioned in the previous section, all the direct cross-section measurements indicate that the interference is constructive. The magnitude of the low-energy peak in the spectrum is sensitive to the value of the reduced-width amplitude in the $\alpha+{ }^{12} \mathrm{C}$ channel for the subthreshold level, assuming that the one in the $\gamma+{ }^{16} \mathrm{O}$ channel, and the $\beta$-feeding amplitude for the state have been fixed by $\gamma$ decay data. When the spectral data were included in some of the recent analyses (Azuma et al., 1994; Buchmann et al., 1996), this sensitivity exaggerated the chi-square differences between the constructive- and destructiveinterference solutions for the cross section enough to rule out the latter solution.

The spectra measured by the TRIUMF (Buchmann et al., 1993; Azuma et al., 1994) and Yale/Connecticut (UConn) (Zhao et al., 1993) groups are generally in good qualitative agreement. However, questions have been raised on both sides about important experimental details, such as the shape of the low-energy peak in the Yale/UConn measurement, the shape of the high-energy peak in the TRIUMF data compared to an earlier measurement (Neubeck, Schober, and Wäffler, 1974), and the effect of target-thickness corrections on comparisons of the two measurements.

Two new measurements of the spectrum have recently been done at the University of Washington in Seattle (Zhao et al., 1995), and at Yale/UConn (France et al., 1997). These new measurements are said to agree well with each other, being as much as a factor of 2 higher than the TRIUMF data in the minimum between the two peaks. This difference could imply that a larger $F$-wave contribution to the spectrum is required in order to fill in the minimum, thus decreasing the $P$-wave contribution (and the extrapolated value of $S_{E 1}$ ) correspondingly. However, Hale (1997) has shown that allowing the $\beta$-feeding amplitude of the positive energy level to have a small imaginary part also has the effect of filling in the minumum between the peaks. Therefore the impact of these new measurements will depend on how they are analyzed.

\section{E2 capture}

The extrapolated E2 capture cross section is also determined by the interaction of positive energy levels (and "direct capture" contributions) with a subthreshold $2^{+}$state at $E=-245 \mathrm{keV}\left(E_{x}=6.9171 \mathrm{MeV}\right)$. In this 
TABLE III. Extrapolated values of $S_{E 2}\left(E_{0}=0.3 \mathrm{MeV}\right)$ obtained from direct measurements of the differential capture cross section.

\begin{tabular}{|c|c|c|}
\hline Reference & $S_{E 2}\left(E_{0}\right)(\mathrm{keV} \mathrm{b})$ & Methods \\
\hline \multirow[t]{3}{*}{ Redder et al. (1987) } & & measured $\sigma(\theta)$; separated $E 1$ and $E 2$ \\
\hline & $96_{-30}^{+24}$ & direct capture + single-level Bréit-Wigner form \\
\hline & $80 \pm 25$ & hybrid $R$ matrix \\
\hline \multirow[t]{2}{*}{ Ouellet et al. (1996) } & & measured $\sigma(\theta)$; separated $E 1$ and $E 2$ \\
\hline & $36 \pm 6$ & microscopic cluster model \\
\hline \multirow[t]{2}{*}{ Trautvetter et al. (1996) } & & measured $\sigma(\theta)$; separated $E 1$ and $E 2$ \\
\hline & $14.5_{-14}^{+96}$ & $\begin{array}{l}R \text { matrix, including } 2^{+} \text {levels below } E_{x}=11.52 \\
\mathrm{MeV}\end{array}$ \\
\hline
\end{tabular}

case, the only broad $2^{+}$level in the region is quite far above the threshold, at $E=4.36 \mathrm{MeV}\left(E_{x}=11.52 \mathrm{MeV}\right)$. In addition, there is a narrow resonance $(\Gamma=0.625 \mathrm{keV})$ at $E_{x}=9.8445 \mathrm{MeV}$ that is not even visible in many of the measurements. The approximate centroid of the E2 strength appears to be at $E_{x}=15 \mathrm{MeV}$, with a spread of about $15 \mathrm{MeV}$. Therefore, one expects the dominant $E 2$ contribution above threshold to come from distant levels and direct capture terms.

The direct measurements lead to extrapolated values of $S_{E 2}(0.3 \mathrm{MeV})$ ranging from 14.5 to $96 \mathrm{keV} \mathrm{b}$, as are shown in Table III. Most of the analyses used some form of microscopic or potential model (usually of Gaussian form) to do the extrapolation, since a pure $R$-matrix or $K$-matrix fit would have involved too many parameters for the number of available data points. In some cases, the potential-model amplitudes were combined with single-level parametrizations of the subthreshold level. However, the present E2 capture data are simply not good enough to allow an unambiguous separation of the resonant and direct effects, even when considered simultaneously with the elastic scattering data.

It is not known with certainty, for example, whether the interference between the subthreshold $2^{+}$state at $E=-245 \mathrm{keV}$ and the distant-level (or "direct") contribution is constructive or destructive. The recent analysis of Trautvetter et al. (1997) is one of the few that has attempted to include the two positive-energy $2^{+}$resonances at $E_{x} \leqslant 11.52 \mathrm{MeV}$. The uncertain nature of the interferences of these levels was a major contributing factor to the rather large uncertainty on the extrapolated $S$ factor listed in Table III. Thus the uncertain role of resonances in the predominantly direct reaction mechanism, and the increased reliance on theoretical models, introduce even more uncertainty into the $E 2$ extrapolation than that for the $E 1$ contribution.

An attempt has been made recently (Kiener et al., 1997) to extract the $E 2$ cross section from the inverse process, Coulomb dissociation of an ${ }^{16} \mathrm{O}$ beam by a ${ }^{208} \mathrm{~Pb}$ target. The data analysis to obtain the equivalent capture cross sections from the $\alpha-{ }^{12} \mathrm{C}$ coincidence spectra is heavily dependent on theoretical calculations, but the qualitative agreement with the capture measurements achieved at this stage shows the promise this method may hold for obtaining new information about the $E 2$ cross section.

\section{Other analyses and recommended values}

A number of analyses not connected with any particular measurement have been done in recent years (Barker, 1987; Filippone et al., 1989; Barker and Kajino, 1991; Humblet et al., 1991) that consider several data sets simultaneously, including the earlier measurement (Neubeck et al., 1974) of the $\alpha$ particle spectrum from the $\beta$ decay of ${ }^{16} \mathrm{~N}$. These analyses often included theoretical refinements of the usual $R$-matrix and $K$-matrix fitting procedures, such as taking into account the channel-region contributions to the photon widths in $R$-matrix theory, and explored the parameter spaces of the representations used. The extrapolated $S$ factors obtained range from 30 to $260 \mathrm{keV} \mathrm{b}$ for $S_{E 1}$, and from 7 to $120 \mathrm{keV}$ b for $S_{E 2}$.

Two recent analyses considered only the available primary data in order to avoid the correlations introduced by using derived quantities (such as elastic-scattering phase shifts) in determining the extrapolated $E 1$ and $E 2$ $S$-factor values. The analysis of Buchmann et al. (1996) included the differential cross-section measurements for ${ }^{12} \mathrm{C}(\alpha, \alpha)$ scattering and ${ }^{12} \mathrm{C}(\alpha, \gamma)$ capture, along with the $\beta$-delayed $\alpha$ spectrum of Azuma et al. (1994). Their results for the $E 1$ part of the cross section essentially confirmed their earlier extrapolated value $S_{E 1}\left(E_{0}\right)=(79 \pm 21) \mathrm{keV} \mathrm{b}$, and for the E2 part, established the upper limit $S_{E 2}\left(E_{0}\right)<140 \mathrm{keV} \mathrm{b}$.

Hale (1996) recently reported an $R$-matrix analysis of the $E 1$ cross section alone that also used the elasticscattering angular-distribution data of Plaga et al. (1987) rather than the $P$ wave scattering phase shift. This analysis indicated that a rather low value of $S_{E 1}\left(E_{0}\right), 20$ $\mathrm{keV} \mathrm{b}$, was consistent with all the measured data, including those of the $\beta$-delayed $\alpha$ spectrum. However, after that analysis was done, the paper of Ouellet et al. (1996) appeared, reporting corrections to earlier data (Ouellet et al., 1992) that significantly raised their low-energy cross sections. Because the uncertainties were so small, their earlier data had been a determining factor in the extrapolated value of $S_{E 1}\left(E_{0}\right)$ obtained by Hale (1996). Therefore, the recent changes in those data will likely raise Hale's extrapolated $E 1 S$ factor above $20 \mathrm{keV} \mathrm{b}$.

At this point, it appears that the best values to recommend for the extrapolated $S$ factors for $\alpha+{ }^{12} \mathrm{C}$ capture are the recent ones of Ouellet et al. (1996). The energy- 


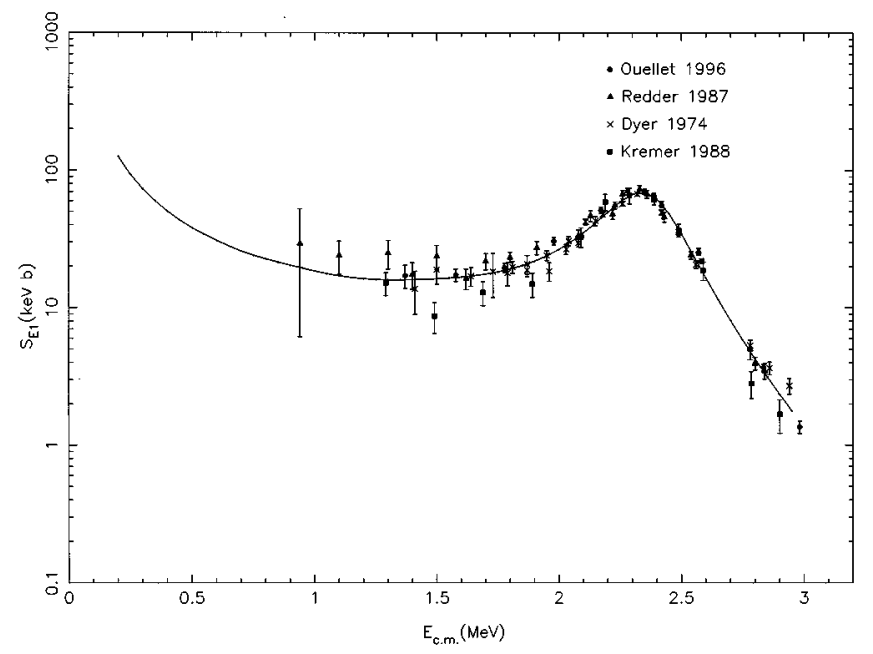

FIG. 4. Experimental $s$ factors of the E1 component of ${ }^{12} \mathrm{C}(\alpha, \gamma){ }^{16} \mathrm{O}$.

dependent $S$ factors from their analysis are shown compared with various direct measurements in Fig. 4 for the $E 1$ component, and in Fig. 5 for the E2 contribution. The curves extrapolate at $E_{0}=300 \mathrm{keV}$ to the values $S_{E 1}\left(E_{0}\right)=(79 \pm 16) \mathrm{keV} \mathrm{b}$ and $S_{E 2}\left(E_{0}\right)=(36 \pm 6) \mathrm{keV} \mathrm{b}$, giving in round numbers $S_{\text {cap }}\left(E_{0}\right)=(120 \pm 40) \mathrm{keV}$ b. To this needs to be added the cascade contributions to $E 2$

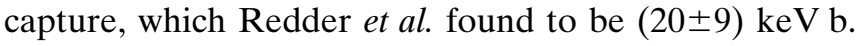
These values are quite consistent with the best estimates obtained by the TRIUMF group, with many of the earlier direct measurements, and with the results of the sensitivity study (Weaver and Woosley, 1993). However, it should be remembered that some of the recent data and analyses continue to indicate lower values of the $E 1 S$ factor. While the uncertainties of these important parameters are gradually decreasing with time, they remain well outside of the $10-15 \%$ level that is desirable for astrophysical applications. Clearly, more work remains to be done on $\alpha+{ }^{12} \mathrm{C}$ capture, especially concerning the extrapolation of the $E 2 S$ factor.

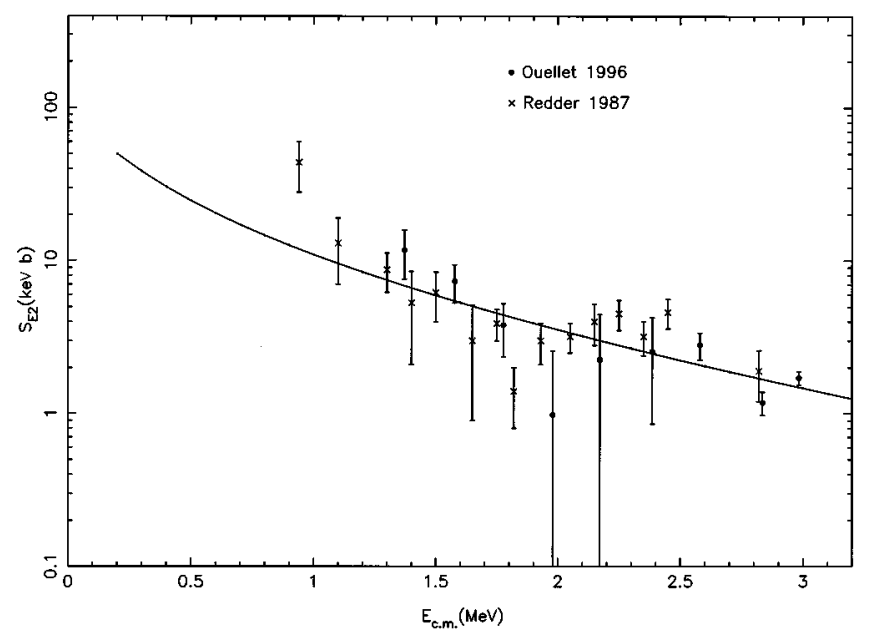

FIG. 5. Experimental $s$ factors of the E2 component of ${ }^{12} \mathrm{C}(\alpha, \gamma){ }^{16} \mathrm{O}$.

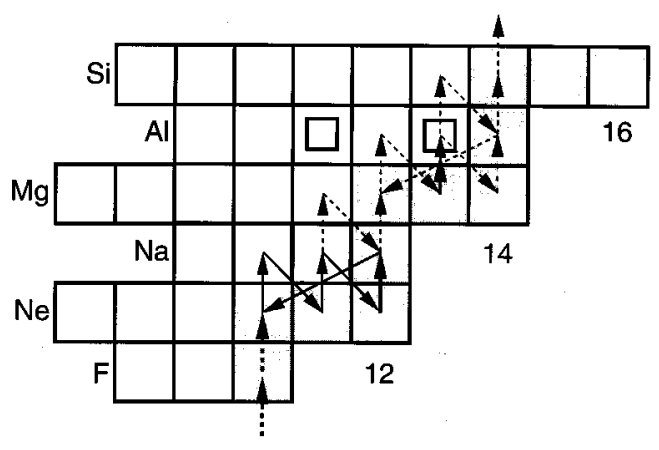

FIG. 6. The $\mathrm{NeNa}$ and $\mathrm{MgAl}$ cycles for $T_{9}=0.05$ and $\rho=100$ $\mathrm{g} / \mathrm{cm}^{3}$. Arrows show integrated fluxes with the heavy lines indicating the strongest flows and the dashed lines representing the weakest. Shaded boxes denote stable nuclei and double boxes indicate the presence of an isomeric state.

\section{H BURNING IN THE NeSi Region: LABORATORY StUdieS}

\section{A. Introduction}

Hydrogen burning beyond the $\mathrm{CNO}$ cycles can begin in the $\mathrm{NeNa}$ cycle and continue into the $\mathrm{MgAl}$ cycle (Fig. 6). The former cycle was described by $\mathrm{B}^{2} \mathrm{FH}$, primarily because they were interested in ${ }^{21} \mathrm{Ne}$ as a potential neutron source. They recognized that since the ${ }^{23} \mathrm{Na}(p, \alpha){ }^{20} \mathrm{Ne}$ reaction is stronger than the competing ${ }^{23} \mathrm{Na}(p, \gamma){ }^{24} \mathrm{Mg}$ reaction, a direct flow to heavier nuclei would be very weak. For this reason, their consideration of hydrogen burning stopped at $\mathrm{Na}$. The years following the publication of $\mathrm{B}^{2} \mathrm{FH}$ have seen (a) the discovery of the ${ }^{22} \mathrm{Ne}$ isotopic anomaly in meteorites (Black, 1972; Lewis et al., 1979; Eberhardt et al., 1979), (b) observations of anticorrelations of $\mathrm{Na}$ and $\mathrm{Al}$ with $\mathrm{O}$ in globular clusters (reviewed by Kraft, 1994 and Kraft et al., 1997), and (c) observations of $\mathrm{Na}$ enhancements in red giant atmospheres (Takeda and Takada-Hidai, 1994). All of these effects are thought to arise from some form of a $\mathrm{NeNa}$ cycle, and so it has remained a topic of interest (additional details concerning $\mathrm{Na}$ and $\mathrm{Al}$ in globular clusters can be found in Sec. VIII of this review). Similarly, the discovery of ${ }^{26} \mathrm{Mg}$ abundance anomalies in meteorites (Lee et al., 1977; Ireland and Compston, 1987; Gallino et al., 1994, Hoppe et al., 1994; Russell et al., 1996), and the discovery of ${ }^{26} \mathrm{Al}$ in the ISM of our galaxy (Mahoney et al., 1984; Share et al., 1985; MacCallum et al., 1987; von Ballmoos et al., 1987, Diehl et al., 1995) have focused a great deal of attention on the $\mathrm{MgAl}$ cycle. The reactions in these two cycles have been subjected to careful experimental scrutiny. Nonetheless, some uncertainties remain as a challenge to nuclear physics. In the following, we review what is known, and what is not known about hydrogen burning in the Ne-Si region.

\section{B. Experimental approaches}

Unlike the situation found in the $\mathrm{CNO}$ cycles, for almost all astrophysical scenarios the cross sections in the 
$\mathrm{NeNa}$ and $\mathrm{MgAl}$ cycles are determined primarily by the contributions of resonances. Although the procedure for measuring these cross sections is rather straightforward in concept, the experiments are difficult in practice because they involve energies well below the Coulomb barrier [see, e.g., Rolfs and Rodney (1988)]. At the lowest energies one must simultaneously produce high quality, high-current proton beams, targets that can withstand the high currents, and efficient detection systems; in many cases, that is a very daunting task.

A large number of resonance strengths has been published for the reactions of interest at lower temperatures. Many of these values result from relative yield measurements that are normalized to the strengths of various standard resonances. This has led to some discrepancies in the literature for cases where the absolute strengths have been in disagreement. For example, several studies have normalized their results to the absolute strengths of Engelbertink and Endt (1966), some of which have since been called into question. A number of more recent absolute measurements have been reported, the most precise being those of Paine and Sargood (1979), Keinonen and Brandenburg (1980), and Anderson et al. (1982), that all quote uncertainties on the order of $10 \%$. These (and other) measurements have provided a consistent and accepted set of absolute strengths. Corrected resonance strengths have been tabulated by Endt (1990, hereafter Endt90), but care must be exercised when referring to the original sources of these data and to collections of reaction rates [e.g., Caughlan and Fowler (1988), hereafter CF88].

The lowest-energy resonance accessible in a direct measurement is ultimately determined by count-rate limitations imposed by the Coulomb barrier. Most of the reactions of the low-temperature $\mathrm{NeNa}$ and $\mathrm{MgAl}$ cycles have been measured directly down to energies on the order of $E_{\text {c.m. }}=150-200 \mathrm{keV}$ which corresponds to stellar temperatures of about $\mathrm{T}_{9}=0.1$ (where the subscript denotes temperature in units of $10^{9} \mathrm{~K}$ ). It does appear possible to extend these measurements to lower energies with the use of modern detection techniques. However, at present the information pertaining to lower-energy resonances has been obtained from indirect nuclear spectroscopy. A simplifying feature at low energies is that the proton partial width, $\Gamma_{p}$, becomes much smaller than those for gamma or alpha emission. Consequently, the resonance strength is proportional to $\Gamma_{p}$, which can be extracted from stripping data via the relation $\Gamma_{p}=C^{2} S \Gamma_{s p}$. Here $C^{2} S$ is the proton spectroscopic factor and $\Gamma_{s p}$ is the proton width for a (ficticious) pure single-particle state. Since these are model-dependent quantities, potentially large systematic errors (factors of 10 or more) could enter into the final results. However, it is possible to check the reliability of this technique by also applying it to measured resonances.

At the high temperatures encountered during explosions, reactions can compete with $\beta$ decay. Consequently, the reaction flow moves to the proton-rich side of stability (Fig. 7) and radioactive beam techniques must be employed to measure the relevant reactions.

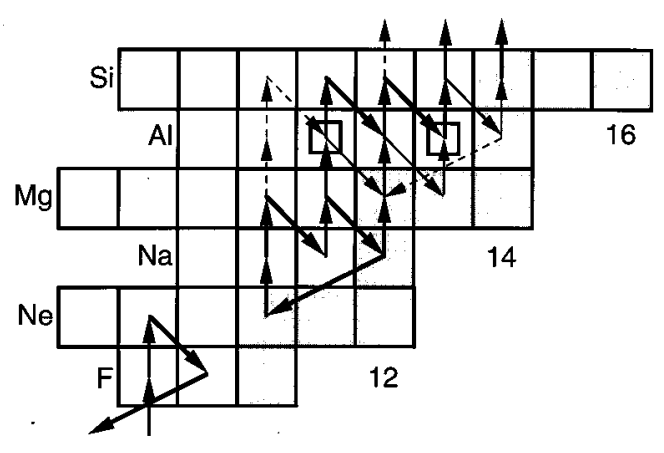

FIG. 7. Integrated fluxes for $T_{9}=0.3$ and $\rho=6000 \mathrm{~g} / \mathrm{cm}^{3}$.

Because reactions will typically occur on time scales that are short when compared with the duration of the explosion, the primary experimental focus is on measuring the rates of reactions that impede the flow to higher masses. The reactions of interest can be identified on the basis of their $(p, \gamma) Q$ values (Iliadis, 1996; see also Rembges et al., 1996). For $Q_{(p, \gamma)} \approx 2-6 \mathrm{MeV}$, the level density is low enough that the reaction rate is composed of the contributions of individual resonances and nonresonant direct capture. Although this in itself does not imply that the reaction is slow, it does mean that the rate is governed by the properties of only a few states. For $Q_{(p, \gamma)}$ below this range, the system can establish a $(p, \gamma)-(\gamma, p)$ equilibrium, in which case the reaction rate is less important. For higher $Q$ values, a large number of resonances may be populated and the reaction can take on a statistical character. Using these criteria, the reactions that are candidates for direct measurements are ${ }^{21} \mathrm{Na}(p, \gamma),{ }^{23} \mathrm{Mg}(p, \gamma)$, and ${ }^{24,25} \mathrm{Al}(p, \gamma)$.

\section{The neon-sodium cycle at low temperatures}

\section{Reaction rates}

All of the reactions of the NeNa cycle have been measured directly and the experimental results may be found in the tabulations by Endt (1990). Reaction rates derived from these measurements are listed in CF88, and little in the way of new experimental information has been presented since then. However, there are significant uncertainties in several of the rates caused by the possible existence of states that could correspond to low-energy resonances. The existing data were recently reevaluated by El Eid and Champagne (1995; hereafter EEC95) who recommended some modifications of the CF88 rates. The experimental situation may be briefly summarized as follows.

(1) The ${ }^{20} \mathrm{Ne}(p, \gamma){ }^{21} \mathrm{Na}$ reaction

At low temperatures, the ${ }^{20} \mathrm{Ne}(p, \gamma)^{21} \mathrm{Na}$ reaction includes an important contribution from the tail of a subthreshold resonance located at $E_{\text {c.m. }}=-6.5 \mathrm{keV}$. The $S$ factors for this state, for the low-energy resonances, and for nonresonant direct capture have been determined by Rolfs et al. (1975). Although $Q_{(p, \gamma)}$ is now $0.5 \mathrm{keV}$ lower (Endt90), EEC95 found that it had little effect on the 
reaction rate. However, this reaction is currently being remeasured (Wiescher, 1996).

(2) The ${ }^{21} \mathrm{Ne}(p, \gamma){ }^{22} \mathrm{Na}$ reaction

Resonances in the ${ }^{21} \mathrm{Ne}(p, \gamma)^{22} \mathrm{Na}$ reaction have been measured (Berg et al., 1977; Keinonen et al., 1977; Görres et al., 1982; Görres et al., 1983; Becker et al., $1992)$ down to $E_{\text {c.m. }}=121 \mathrm{keV}$. Two lower-energy states exist and uncertainties concerning their single-particle strengths led to large uncertainties in the rate tabulated in CF88. Limits on the proton spectroscopic factors for these states, based upon available data, were deduced by EEC95. They predict that the two low-energy states should have an almost negligible effect on the rate. The current lower limit on the rate is very nearly the same as the upper limit appearing in CF88.

(3) The ${ }^{22} \mathrm{Na}(p, \gamma){ }^{23} \mathrm{Mg}$ reaction

Although ${ }^{22} \mathrm{Na}$ is radioactive, it could provide a pathway for leakage out of the $\mathrm{NeNa}$ cycle in situations where the $(p, \gamma)$ lifetime is comparable to the lifetime for $\beta^{+}$decay (3.75 y). Schmidt et al. (1995) have used the ${ }^{22} \mathrm{Na}\left({ }^{3} \mathrm{He}, d\right)$ reaction to determine the strengths of low-energy resonances and direct $(p, \gamma)$ measurements have also been performed by Seuthe et al. (1990) and by Stegmüller et al. (1996). For pure hydrogen at a density of $10^{3} \mathrm{~g} / \mathrm{cm}^{3}$, the $\beta^{+}$decay and $(\mathrm{p}, \gamma)$ lifetimes are equal for temperatures $\mathrm{T}_{9}=0.039-0.068$, where the range in temperature reflects the uncertainty in the reaction rate. Note that this density is high for some low-temperature sites. At $10 \mathrm{~g} / \mathrm{cm}^{3}$, the transition occurs for $\mathrm{T}_{9}=0.08-0.1$.

(4) The ${ }^{22} \mathrm{Ne}(p, \gamma)^{23} \mathrm{Na}$ reaction

Unfortunately, the rate of the ${ }^{22} \mathrm{Ne}(p, \gamma)^{23} \mathrm{Na}$ reaction is rather poorly known at low temperatures because it appears that 13 states exist in the region between threshold and the first measured resonance (Endt90). Two of these states have been observed in $\left({ }^{3} \mathrm{He}, d\right)$ studies and the appropriate resonance strengths have been calculated by EEC95. Further $(p, \gamma)$ studies by Görres et al. (1982) and Görres et al. (1983) have indicated that perhaps two of the remaining eleven states might be astrophysically significant, but it is not clear that these states exist, and it is certainly not possible to place stringent limits on their resonance strengths. This situation leads to an uncertainty in the reaction rate of a factor of 23400 for $\mathrm{T}_{9}=0.03-0.08$. Although it is most likely that the actual rate is near its current lower limit, it is presently the most uncertain rate in the $\mathrm{NeNa}$ cycle.

(5) The ${ }^{23} \mathrm{Na}(p, \gamma){ }^{24} \mathrm{Mg}$ and ${ }^{23} \mathrm{Na}(p, \alpha){ }^{20} \mathrm{Ne}$ reactions

Resonances in the ${ }^{23} \mathrm{Na}+p$ system have been measured down to $E_{\text {c.m. }}=171 \mathrm{keV}$ (Zyskind et al., 1981; Görres et al., 1989), but again, the possibility exists that there are other resonances between this level and threshold that may be important at low temperatures. Crude estimates of spectroscopic factors were given by EEC95. In the case of the $(p, \alpha)$ reaction, large uncertainties exist, but only for $\mathrm{T}_{9} \leqslant 0.03$, whereas the $(p, \gamma)$ reaction is uncertain by about a factor of 2 for a narrow range of temperatures near $\mathrm{T}_{9}=0.08$. Although better experimental information is desirable, it is clear that the $(p, \alpha)$ is much faster than the $(p, \gamma)$ reaction at low temperatures and therefore the $\mathrm{NeNa}$ cycle will in fact cycle.

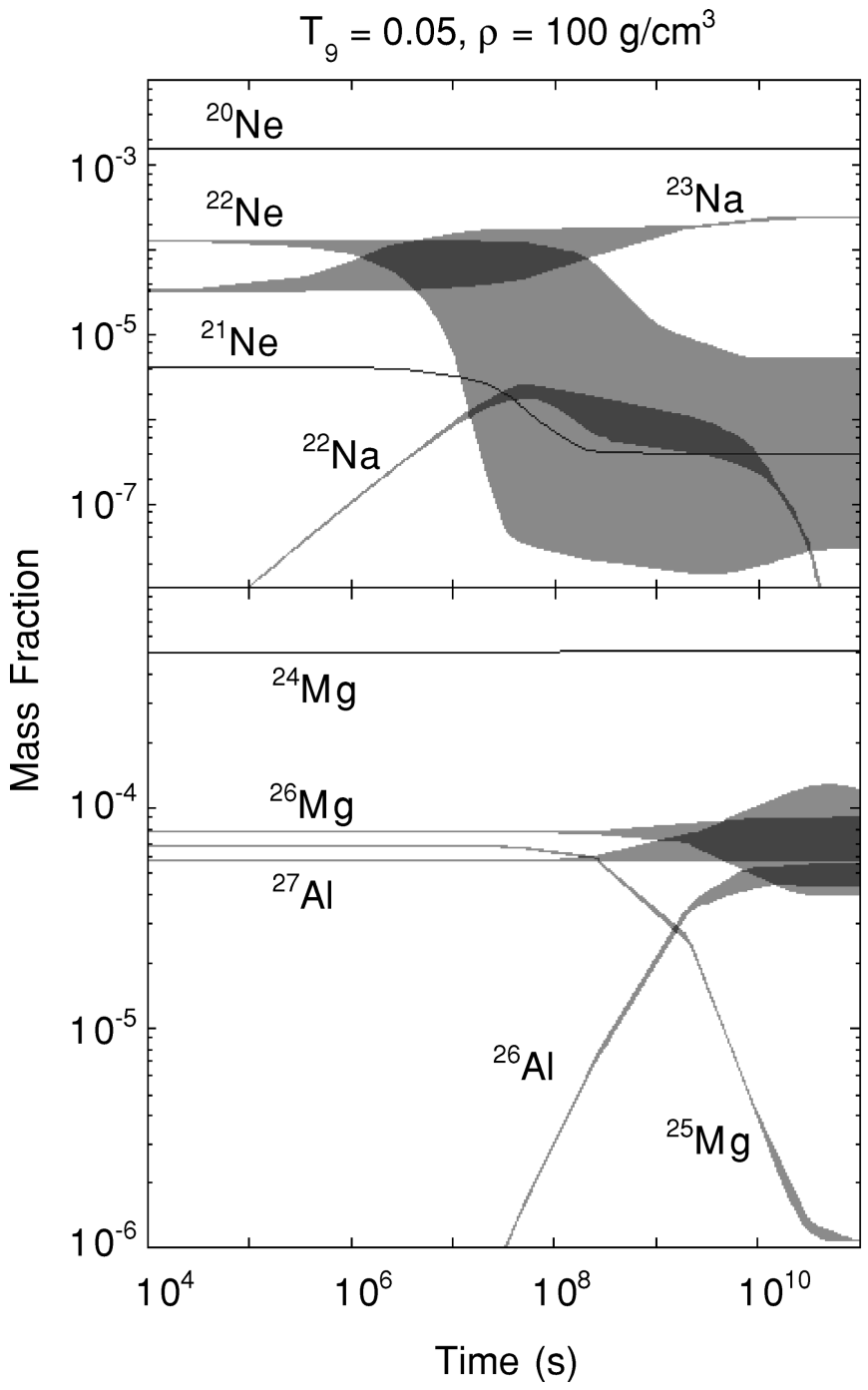

FIG. 8. Time evolution of mass fractions in the $\mathrm{NeNa}$ and $\mathrm{MgAl}$ cycle for $T_{9}=0.05, \rho=100 \mathrm{~g} / \mathrm{cm}^{3}$, and solar initial composition. Shaded areas indicate the range of values allowed by the upper and lower limits on the individual reaction rates.

The situation is reversed for temperatures $\mathrm{T}_{9}=0.18-0.6$, but as the temperature increases, the reaction flow will begin to leave the $\mathrm{NeNa}$ cycle via the ${ }^{22} \mathrm{Na}(p, \gamma)$ reaction, bypassing ${ }^{23} \mathrm{Na}$.

\section{Network calculations}

Shown in Fig. 8 are the results of network calculations performed for $\mathrm{T}_{9}=0.05$, assuming a constant density of $100 \mathrm{~g} / \mathrm{cm}^{3}$, and solar initial abundances [other calculations may be found in Arnould et al. (1995)]. Although, these are artificial and rather generic parameters, the results should illustrate what might be expected from the $\mathrm{NeNa}$ cycle in red giants, for example. The most striking features of Fig. 8 are the almost negligible depletion of ${ }^{20} \mathrm{Ne}(5 \%)$ and the strong destruction of ${ }^{22} \mathrm{Ne}$ which together lead to an enrichment in ${ }^{23} \mathrm{Na}$ by about a factor of 7 . Because ${ }^{21} \mathrm{Ne}$ is rapidly destroyed, the uncertainty in the ${ }^{21} \mathrm{Ne}(p, \gamma)$ reaction rate has little effect on the final abundances. The first branch point in 
the cycle appears at ${ }^{22} \mathrm{Na}$. At its upper limit, the ${ }^{22} \mathrm{Na}(p, \gamma)$ rate is about 3 times faster than the rate for $\beta^{+}$decay. This leads to a gradual production of ${ }^{23} \mathrm{Mg}$, but since this decays rapidly to ${ }^{23} \mathrm{Na}$, the net effect of the ${ }^{22} \mathrm{Na}(p, \gamma)$ reaction is just to bypass ${ }^{22} \mathrm{Ne}(p, \gamma)$. Since the latter reaction is also comparatively fast, this has little effect on the final abundance of ${ }^{23} \mathrm{Na}$. For similar reasons, the large uncertainty in the ${ }^{22} \mathrm{Ne}(p, \gamma)$ rate does not translate into a comparable uncertainty for ${ }^{23} \mathrm{Na}$. The overall cycle time $(9160 \mathrm{y}$ for these conditions) is determined by the rates of the slowest reactions, namely ${ }^{20} \mathrm{Ne}(p, \gamma)$ and ${ }^{23} \mathrm{Na}(p, \alpha)$, and at $\mathrm{T}_{9}=0.05$ these rates are known rather precisely. Because the ${ }^{23} \mathrm{Na}(p, \alpha)$ reaction is about 18 times faster than the competing ${ }^{23} \mathrm{Na}(p, \gamma)$ reaction, the $\mathrm{NeNa}$ cycle is essentially closed (though this will not be the case at higher temperatures and densities, as will be seen below).

\section{The magnesium-aluminum cycle at low temperatures}

\section{Reaction rates}

The origin of the radioactive isotope ${ }^{26} \mathrm{Al}$ became an interesting problem in astrophysics with the discovery of an excess of its daughter species, ${ }^{26} \mathrm{Mg}$, in the Allende meteorite (Lee et al., 1977). The presence of a $\gamma$ ray line due to the decay of ${ }^{26} \mathrm{Al}$ to ${ }^{26} \mathrm{Mg}$ in the Galaxy pointed to the production of ${ }^{26} \mathrm{Al}$ in the Galaxy at the present time, since the half-life of ${ }^{26} \mathrm{Al}$ is $7.2 \times 10^{5}$ years. In addition the discovery of an excess of $\mathrm{Al}$ (presumably ${ }^{27} \mathrm{Al}$ ) in globular cluster stars that are deficient in oxygen and show an excess of $\mathrm{Na}$ (Kraft et al., 1997) added impetus to efforts to understand the $\mathrm{MgAl}$ cycle. Although uncertainties in the $\mathrm{MgAl}$ cycle are somewhat smaller than those for the NeNa cycle, some further investigation is warranted. Unfortunately, most rate compilations predate some of the more definitive experimental results. Therefore in the following we have summarized the current status of these reactions.

(1) The ${ }^{24} \operatorname{Mg}(p, \gamma){ }^{25} \mathrm{Al}$ reaction

The ${ }^{24} \operatorname{Mg}(p, \gamma){ }^{25} \mathrm{Al}$ reaction has been studied rather extensively by Trautvetter and Rolfs (1975) and Trautvetter (1975). The reaction rate found in CF88 is based upon these results and appears to be reliable. However, it has been suggested (Cavallo et al., 1996; Shetrone, 1996a, 1996b) that the rate must be higher than believed in order to account for trends of $\mathrm{Mg}$ vs $\mathrm{Al}$ observed in globular clusters. Further work on this reaction is in progress (Iliadis, 1996a).

(2) $\mathrm{The}^{25} \mathrm{Mg}(p, \gamma){ }^{26} \mathrm{Al}$ reaction

Surveys of resonances in the ${ }^{25} \mathrm{Mg}(p, \gamma){ }^{26} \mathrm{Al}$ reaction have been reported (Elix et al., 1979; Anderson et al., 1980; Endt et al., 1986; Iliadis et al., 1990) and results are tabulated in Endt90. A search for possible low-energy resonances was undertaken by Champagne et al. (1983) using data obtained via the ${ }^{25} \mathrm{Mg}\left({ }^{3} \mathrm{He}, d\right)$ reaction. In order to eliminate the model dependence associated with calculations of single-particle widths, they obtained proton widths by normalizing to higher energy resonances, where both proton widths and spectroscopic fac- tors could be measured. Unfortunately, some of the conclusions that they drew from their data were in error. Motivated by the ${ }^{25} \mathrm{Mg}(p, \gamma \gamma)$ studies of Endt et al. (1986), improved, higher-resolution $\left({ }^{3} \mathrm{He}, d\right)$ measurements were performed (Champagne et al., 1986a) that produced a more reliable set of spectroscopic factors and reaction rates. A notable outcome of this work was the identification of a strong s-wave resonance at an energy of $E_{\text {c.m. }}=58 \mathrm{keV}$ which substantially increased the production of ${ }^{26} \mathrm{Al}$ at low temperatures. However, Endt and Rolfs (1987) calculated reaction rates that were in disagreement with these results, primarily because of their use of square-well single-particle widths, but also because they overestimated several important spectroscopic factors. The situation was clarified by Champagne et al. (1989) and Rollefson et al. (1990) who produced rates consistent with one another and with Champagne et al. (1986a) while also extending their analyses to include weakly populated states. Finally, Iliadis et al. (1996) derived reaction rates from the existing data by employing a realistic diffuse optical model potential in the calculation of single particle widths. Although the results obtained were virtually identical to those of Champagne et al. (1989), the treatment of single particle widths by Iliadis et al. (1996) has the advantage of selfconsistency. Though laborious, this approach appears to be the best method for extracting resonance parameters from stripping data.

Proton capture will produce ${ }^{26} \mathrm{Al}$ in either its longlived ground state $\left({ }^{26} \mathrm{Al}_{0}, T_{1 / 2}=7.2 \times 10^{5} \mathrm{y}\right)$ or in its short-lived first excited state $\left({ }^{26} \mathrm{Al}_{1}, 6.35 \mathrm{~s}\right)$ and these states will not equilibrate at low temperatures (Ward and Fowler, 1980). Therefore, gamma ray branching ratios (Endt and Rolfs, 1987) must be employed in order to separate the total reaction rate into separate contributions for each state. The most recent evaluations of these rates is given by Iliadis et al. (1996) and the results are similar to the tabulation found in Iliadis et al. (1990). However, they are significantly smaller (by factors of 3 to 6 for $\mathrm{T}_{9}=0.05-0.1$ ) than the rates found in CF88.

(3) The ${ }^{26} \mathrm{Mg}(p, \gamma){ }^{27} \mathrm{Al}$ reaction

Low-energy resonances are also found to make important contributions to the rate of the ${ }^{26} \mathrm{Mg}(p, \gamma){ }^{27} \mathrm{Al}$ reaction. States corresponding to resonances below 105 $\mathrm{keV}$ have been detected using the ${ }^{26} \mathrm{Mg}\left({ }^{3} \mathrm{He}, d\right)$ reaction (Champagne et al., 1990). Unfortunately, only an upper limit was obtained for the strength of a possible $90 \mathrm{keV}$ resonance. The resulting uncertainty in the reaction rate is a factor of 37 at $\mathrm{T}_{9}=0.04$ and grows to a factor of 74 at $\mathrm{T}_{9}=0.06$ before falling at higher temperatures. This is particularly troublesome because it directly affects predictions of the the ${ }^{26} \mathrm{Al} /{ }^{27} \mathrm{Al}$ production ratio. Further measurements are certainly called for. Direct $(p, \gamma)$ measurements have also been carried out (Buchmann et al., 1980; Keinonen and Brandenburg, 1980; Iliadis et al., 1990), and in contrast to the situation at lower temperatures, the rate at higher temperatures (i.e., $\mathrm{T}_{9} \geqslant 0.1$ ) is well established. The rate given in CF88 was based upon preliminary results for the low-energy reso- 
nances and differs by a factor of $0.45-2$ from the present upper limit appropriate for temperatures $\mathrm{T}_{9}=0.05-0.1$.

(4) $\mathrm{The}^{26} \mathrm{Al}_{0}(p, \gamma){ }^{27} \mathrm{Si}$ reaction

Initial measurements of the ${ }^{26} \mathrm{Al}_{0}(p, \gamma)^{27} \mathrm{Si}$ reaction were carried out by Buchmann et al. (1984) and a more complete investigation was made by Vogelaar (1989). However, the rate for $\mathrm{T}_{9} \leqslant 0.15$ is quite uncertain, again because of the possibility of several unobserved lowenergy resonances. None of these states were detected in $\left({ }^{3} \mathrm{He}, d\right)$ measurements (Vogelaar, 1989, Vogelaar et al., 1996), but they have been treated theoretically by Champagne et al. (1993). Although the results of these calculations seem justified, there is little in the way of experimental information (in particular, spins, parities, or spectroscopic factors) that would support them. Improved $\left({ }^{3} \mathrm{He}, d\right)$ studies, using more highly enriched ${ }^{26} \mathrm{Al}$ targets will be needed before uncertainties in this rate can be reduced.

(5) The ${ }^{27} \mathrm{Al}(p, \gamma){ }^{28} \mathrm{Si}$ and ${ }^{27} \mathrm{Al}(p, \alpha){ }^{24} \mathrm{Mg}$ reactions

Once ${ }^{27} \mathrm{Al}$ is synthesized, a $(p, \alpha)$ reaction may act to close the $\mathrm{MgAl}$ cycle, or alternatively, the reaction flow will proceed to higher masses via a $(p, \gamma)$ reaction. Resonances at $E_{\text {c.m. }}=72$ and $85 \mathrm{keV}$ (Endt90) could play a role in both reactions at low temperatures, but again they lie too low in energy to be observed in proton capture. The corresponding states in ${ }^{28} \mathrm{Si}$ were studied via $\left({ }^{3} \mathrm{He}, d\right)$ and $(\alpha, t)$ spectroscopy (Champagne et al., 1986b) and their particle decays have also been measured (Champagne et al., 1988). Although the original study (Champagne et al., 1986b) questioned whether either state possessed any single particle strength, the magnitude and shape of the $\left({ }^{3} \mathrm{He}, d\right)$ angular distribution for the lower state may imply a single particle contribution. Consequently, the rate used in the review of Arnould et al. (1995) includes a lower limit for the strength of the $72-\mathrm{keV}$ resonance. As a result, the possible $85-\mathrm{keV}$ resonance was found to be too weak to be of astrophysical significance.

Between these low-energy states and the known $(p, \alpha)$ resonance at $E_{\text {c.m. }}=487 \mathrm{keV}$ lie several states that have been observed in both the ${ }^{27} \mathrm{Al}(p, \gamma){ }^{28} \mathrm{Si}$ and ${ }^{24} \mathrm{Mg}$ $(\alpha, \gamma){ }^{28} \mathrm{Si}$ reactions. This means that they must be resonances in the ${ }^{27} \mathrm{Al}(p, \alpha){ }^{24} \mathrm{Mg}$ reaction. Limits on their strengths from direct $(p, \alpha)$ measurements (Timmermann et al., 1988) and complementary information from measurements of particle decays (Champagne et al., 1988) indicate that all can be ignored except for resonances at 196 and $215 \mathrm{keV}$. However, the strengths of these two resonances are unknown and this is a major source of uncertainty in the reaction rate. Subthreshold resonances may also be ignored [though note that the upper limits quoted by Timmermann et al. (1988) are in fact lower limits]. The strengths of higher energy resonances are listed in Endt90.

The reaction rate is uncertain by about a factor of six for $\mathrm{T}_{9}<0.07$, primarily because of the upper and lower limits on the strength of the $72-\mathrm{keV}$ resonance. At higher temperatures, the uncertainty grows dramatically as the 196 and $215 \mathrm{keV}$ resonances come into play. As was discussed by Champagne et al. (1988), the rate ap-

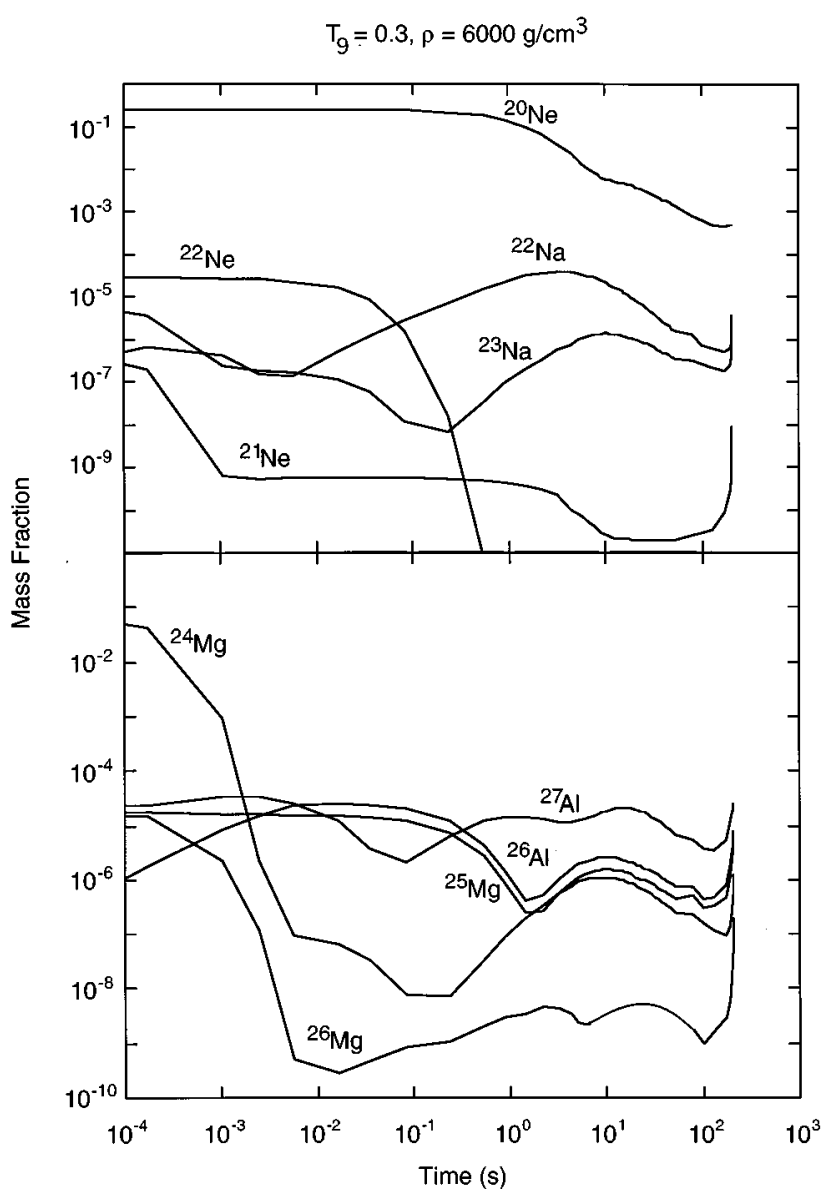

FIG. 9. Time evolution of mass fractions for $T_{9}=0.3, \rho=6000$ $\mathrm{g} / \mathrm{cm}^{3}$, and $\mathrm{O}-\mathrm{Ne}$ initial abundances.

pearing in CF88 is based in part on erroneous data and hence bears little resemblance to the rate derived from current information.

From these same data sets it is possible to obtain the rate for the ${ }^{27} \mathrm{Al}(p, \gamma){ }^{28} \mathrm{Si}$ reaction. Beyond $\mathrm{T}_{9}=0.07-0.08$, the $(p, \gamma)$ reaction is much faster than the competing $(p, \alpha)$ reaction. For lower temperatures, the $(p, \alpha)$ reaction is faster by about a factor of 4 , but the cycle time is significant. Hence the $\mathrm{MgAl}$ reactions will behave more like a reaction chain than a reaction cycle.

\section{Network calculations}

Network calculations for the $\mathrm{MgAl}$ cycle have been performed for the same conditions discussed above for the NeNa cycle and results are displayed in Fig. 9. The main effect of the $\mathrm{MgAl}$ cycle at low temperatures is the production of ${ }^{26} \mathrm{Al}$ at the expense of ${ }^{25} \mathrm{Mg}$. This also leads to ${ }^{26} \mathrm{Mg}$ production following the $\beta^{+}$decay of ${ }^{26} \mathrm{Al}_{1}$. If the rate for the ${ }^{26} \mathrm{Mg}(p, \gamma)$ reaction is near its present upper limit, then ${ }^{26} \mathrm{Mg}$ suffers a net depletion of a factor of 2.3 while ${ }^{27} \mathrm{Al}$ increases by a similar factor. Neither the ${ }^{27} \mathrm{Al}(p, \gamma)$ nor the ${ }^{27} \mathrm{Al}(p, \alpha)$ reaction has much effect on the final abundance of ${ }^{27} \mathrm{Al}$ since both reaction times are longer than the time required to deplete hydrogen (in this simulation). Hence the $\mathrm{MgAl}$ 
cycle acts as individual reactions terminating in ${ }^{26,27} \mathrm{Al}$. Rather high ${ }^{26} \mathrm{Al} /{ }^{27} \mathrm{Al}$ ratios are achieved $(0.38-0.95)$. The uncertainty in this ratio stems primarily from the uncertainty in the ${ }^{26} \mathrm{Mg}(p, \gamma)$ rate and, to a lesser extent, from ${ }^{26} \mathrm{Al}(p, \gamma)$.

\section{E. High-temperature behavior}

\section{Reaction rates}

For temperatures in excess of $\mathrm{T}_{9}=0.2$, nuclear burning proceeds in more or less a continuous flow through the $\mathrm{Ne}-\mathrm{Si}$ region to higher masses. The distinguishing feature of this scenario is that the reaction path is moved to the proton-rich side of stability. As was shown in Fig. 7, a number of $(p, \gamma)$ reactions involve short-lived nuclei, namely ${ }^{21} \mathrm{Na},{ }^{22,23} \mathrm{Mg}$, and ${ }^{23,25} \mathrm{Al}$. Since these reactions have not been measured directly, we presently rely upon estimated reaction rates.

A number of authors (Wiescher and Langanke, 1986; Wiescher et al., 1986; Wiescher et al., 1988, van Wormer et al., 1994; Herndl et al., 1995; Iliadis et al., 1996b) have examined the reactions in this mass region and have used similar procedures for obtaining resonance parameters. All make use of the known properties of states in stable isobars. In some cases, analog assignments were complicated by the fact that the states of interest, expected on the basis of the level structure in the analog nucleus, had not been observed and shell-model calculations were used in order to predict their locations. Proton widths were calculated from both experimental and theoretical single-particle spectroscopic factors. In the case of the ${ }^{21} \mathrm{Na}(p, \gamma)$ reaction, the magnitude of the energy shift between analogous states in ${ }^{22} \mathrm{Mg}$ and ${ }^{22} \mathrm{Na}$ was used to calculate single-particle reduced widths (Wiescher and Langanke, 1986). This procedure assumes that the states in question are primarily pure single-particle states and this must be justified on a state by state basis. For the ${ }^{22} \mathrm{Mg}(p, \gamma)$ reaction, estimated spectroscopic factors were initially employed (Wiescher et al., 1986), but were later improved upon by using shell-model predictions (Wiescher et al., 1988). With the ${ }^{23} \mathrm{Mg}(p, \gamma)$ and ${ }^{25} \mathrm{Al}(p, \gamma)$ reactions, it was possible to make use of measured spectroscopic factors, obtained from ${ }^{23} \mathrm{Na}(d, p)$ and ${ }^{25} \mathrm{Mg}(d, p)$, respectively (Wiescher et al., 1986, Iliadis et al., 1996b). Finally, in the case of the ${ }^{23,24} \mathrm{Al}(p, \gamma)$ reactions, spectroscopic factors were estimated from assumed nuclear structure systematics (van Wormer et al., 1994), and from shell-model calculations (Herndl et al., 1995).

Values for $\gamma$ widths were obtained from values for typical $\gamma$ ray transition strengths. The latter resulted from shell-model calculations of $B(E 2)$ factors as well as from simple estimates based upon the known lifetimes of the analog states. Because changes in excitation energies or widths may easily change a reaction rate by orders of magnitude, it is clear that the rates for these reactions must be periodically reexamined as new experimental information becomes available.

\section{Network calculations}

Network calculations were again performed for constant temperature and density, in this case $\mathrm{T}_{9}=0.3$ and $\rho=6000 \mathrm{~g} / \mathrm{cm}^{2}$. These conditions might be encountered at the peak of a nova outburst on an O-Ne white dwarf. Initial abundances were taken from Politano et al. (1995). Abundances were tracked until just after hydrogen exhaustion, which occurred after about 200 s. Note that the time scale is set by the rate of hydrogen consumption in the $\mathrm{CNO}$ region rather than in the $\mathrm{Ne}-\mathrm{Si}$ region. Also, it should again be emphasized that this calculation represents an artificial situation. Nonetheless, it should illustrate some of the behavior that would be found in a more realisitic treatment. The results are shown in Fig. 9.

Burning starts with a rapid decline in ${ }^{21} \mathrm{Ne}$. This is followed by a period of near equilibrium between ${ }^{21} \mathrm{Ne}$ production [from ${ }^{20} \mathrm{Ne}(p, \gamma)^{21} \mathrm{Na}\left(\beta^{+}\right)^{21} \mathrm{Ne}$ ] and destruction via the ${ }^{21} \mathrm{Ne}(p, \gamma)$ reaction. This situation persists until the abundance of ${ }^{20} \mathrm{Ne}$ starts to drop (at about 10 s) which slows the production of ${ }^{21} \mathrm{Ne}$. The equilibrium at ${ }^{21} \mathrm{Ne}$ results in a slow increase in the ${ }^{22} \mathrm{Na}$ abundance. As ${ }^{21} \mathrm{Ne}$ falls out of equilibrium and is destroyed, the ${ }^{22} \mathrm{Na}$ abundance also falls as its production can no longer keep pace with the ${ }^{22} \mathrm{Na}(p, \gamma)$ reaction. Similar behavior is observed for ${ }^{23} \mathrm{Na}$. Its increased abundance after $1 \mathrm{~s}$ is a consequence of the $\beta^{+}$decay of ${ }^{23} \mathrm{Mg}$ following the ${ }^{22} \mathrm{Na}(p, \gamma)$ reaction. The decrease in ${ }^{22} \mathrm{Na}$ following ${ }^{20} \mathrm{Ne}$ slows production of ${ }^{23} \mathrm{Na}$ while it is being destroyed by ${ }^{23} \mathrm{Na}(p, \gamma)$ and ${ }^{23} \mathrm{Na}(p, \alpha)$. After hydrogen exhaustion, the fast $\beta^{+}$decays of ${ }^{21} \mathrm{Na}$ and ${ }^{22,23} \mathrm{Mg}$ produce rapid increases in the abundances of ${ }^{21} \mathrm{Ne}$ and ${ }^{22,23} \mathrm{Na}$.

The abundance of ${ }^{24} \mathrm{Mg}$ displays a rapid initial drop from ${ }^{24} \operatorname{Mg}(p, \gamma)$ and later production after $\beta^{+}$decay of ${ }^{24} \mathrm{Al}$. The ${ }^{25} \mathrm{Mg}$ abundance is initially nearly flat as the ${ }^{25} \mathrm{Mg}(p, \gamma)$ is almost offset by production of ${ }^{25} \mathrm{Mg}$ through the $\beta^{+}$decay of ${ }^{25} \mathrm{Al}$. However, this causes significant production of ${ }^{26} \mathrm{Al}$ which for a short time is the second-most abundant nuclide in the $\mathrm{MgAl}$ region (after ${ }^{24} \mathrm{Mg}$ ). At these temperatures and densities, the time required for ${ }^{26} \mathrm{Al}_{0}$ to come into equilibrium with ${ }^{26} \mathrm{Al}_{1}$ is long compared with the overall burning time and thus these two levels may still be considered independently (Ward and Fowler, 1980). Following this period, ${ }^{26} \mathrm{Al}$ evolves in parallel with ${ }^{25} \mathrm{Mg}$. The drop in ${ }^{24} \mathrm{Mg}$ diminishes production of ${ }^{25} \mathrm{Mg}$ and results in a decrease in both ${ }^{25} \mathrm{Mg}$ and ${ }^{26} \mathrm{Al}$. This trend is reversed for a short time following the production of ${ }^{24} \mathrm{Mg}$, as a result of material moving out of the $\mathrm{NeNa}$ region. The ${ }^{27} \mathrm{Al}$ abundance shows the effects of competition between the ${ }^{26} \mathrm{Mg}(p, \gamma)$ reaction, and the ${ }^{27} \mathrm{Al}(p, \gamma)$ and ${ }^{27} \mathrm{Al}(p, \alpha)$ reactions. The $\beta^{+}$decay of ${ }^{26} \mathrm{Al}_{1}$ produces a slow rise in ${ }^{26} \mathrm{Mg}$ followed by a sharp increase after burning has ceased. Similar effects are observed for ${ }^{24,25} \mathrm{Mg}$ and ${ }^{26,27} \mathrm{Al}$.

As was mentioned above, under these conditions the reaction network is characterized by a flow through the $\mathrm{Ne}-\mathrm{Si}$ mass region rather than by reaction cycles. Only 
about $0.2 \%$ (by mass) of the initial $\mathrm{NeNa}$ material survives, primarily as ${ }^{20} \mathrm{Ne}$. About $0.04 \%$ of the $\mathrm{MgAl}$ material remains, mostly as ${ }^{27} \mathrm{Al}$.

\section{F. Conclusion}

Many of the developments that have lead to our continuing interest in the $\mathrm{NeNa}$ and $\mathrm{MgAl}$ cycles could not have been anticipated at the time of $\mathrm{B}^{2} \mathrm{FH}$, but are nonetheless direct descendents of this initial framework. It is possible that there are aspects to nucleosynthesis in this mass region that remain to be uncovered. It is clear that there is still a significant amount of experimental and theoretical nuclear astrophysics to be done before we will understand such things as the origin of ${ }^{26} \mathrm{Al}$ and the mechanism behind the $\mathrm{Na} / \mathrm{Al}$ abundance anomalies in meteorites. Many reaction rates are still uncertain, particularly at the extremes of temperature. For low temperatures, the problems seem tractable. However, calculations of nucleosynthesis at high temperatures require a wealth of information, such as masses, lifetimes, and decay schemes, in addition to reaction rates. Collecting these data requires the development of new experimental techniques, for example, the use of radioactive beams. Gaining insight from them will entail the construction of increasingly sophisticated models.

\section{OBSERVATIONAL EVIDENCE OF HYDROGEN BURNING}

There can be no denying that hydrogen burning provides the primary source of energy in the sun. No other process could maintain the sun's luminosity of $4 \times 10^{33}$ ergs $\mathrm{sec}^{-1}$ over a time scale of $4.5 \times 10^{9}$ years (Bethe, 1939). Although the neutrino spectrum from the sun has shown certain discrepancies when compared to predictions (see Introduction), these problems are likely to be solved as our understanding of both solar physics and neutrino physics improves (Haxton, 1995).

Since hydrogen burning dominates the energy generation of main-sequence stars and their early evolution it is worthwhile to mention some aspects of their evolution to supplement Sec. III of this review. Stars of more than about $20 M_{\odot}$ suffer mass loss at a rate that depends sensitively on the mass, reaching $10^{-5} M_{\odot} \mathrm{yr}^{-1}$ for masses near $50 M_{\odot}($ Cassinelli, 1979). The mass loss is so substantial that the radiative outer layers may be lost before the core has converted its $\mathrm{H}$ to He. As the products of nucleosynthesis reach the stellar surface the star first becomes an OB-peculiar star with excessive N (Walborn, 1987) and then a Wolf-Rayet star whose surface composition is dominated by $\mathrm{He}$ and $\mathrm{N}$. The latter species is greatly enhanced by the conversion of $\mathrm{C}$ and $\mathrm{O}$ during the CNO bi-cycle (Abbott and Conti, 1987). During their further evolution with an increasing mass-loss rate their $\mathrm{He}$ and $\mathrm{N}$ enriched envelope is lost and they become Wolf-Rayet stars of the carbon type, revealing the products of helium burning reactions.

Further down the main sequence (near $0.8 M_{\odot}$ ) are the stars in globular clusters that are evolving off the main-sequence after spending about $(11-13) \times 10^{9}$ years burning $\mathrm{H}$ in their radiative cores (Reid, 1997). Our understanding of the evolution of globular cluster stars has been vastly developed since the groundbreaking paper of Hoyle and Schwarzschild (1955) and has most recently been reviewed by Vandenberg et al. (1996). After the evolving star has crossed the subgiant branch its luminosity increases rapidly as the outer convection zone penetrates to greater depths and begins to dredge up material that had been hot enough for the $\mathrm{CN}$ cycle to convert C to $\mathrm{N}$ (Iben, 1967a). This process is commonly called the "first dredge-up."

As the star evolves up the red giant branch, additional mixing occurs (to be discussed later in this section) until the now degenerate core reaches about $80 \times 10^{6} \mathrm{~K}$ and the triple-alpha reaction triggers the "helium flash." The star then settles down on the horizontal branch of the HR diagram burning helium in its core and hydrogen in a shell that surrounds the core. When the helium in the core is depleted the star brightens along the asymptotic giant branch as described by Iben in Sec. III of this review.

Hydrogen burning has successfully predicted many phenomena that have been seen in the changes of chemical composition of the observable surface layers of stars. Two examples of the comparison of stellar observations with the theory of stellar structure, nuclear reaction rates, and mixing processes are the ratio of ${ }^{12} \mathrm{C} /{ }^{13} \mathrm{C}$ and the observed excesses of $\mathrm{Na}$ and $\mathrm{Al}$ in globular cluster stars. We first discuss the change of the ${ }^{12} \mathrm{C} /{ }^{13} \mathrm{C}$ ratios in stars as they evolve from the main sequence to the first-ascent giant branch. As stars advance up the red giant branch the convection zone deepens until it dredges up material in which both ${ }^{12} \mathrm{C}(p, \gamma){ }^{13} \mathrm{~N}\left(\beta^{+} \nu\right){ }^{13} \mathrm{C}$ and ${ }^{13} \mathrm{C}(p, \gamma){ }^{14} \mathrm{~N}$ had been active (Iben, 1967a; Dearborn et al., 1976; Sweigert et al., 1989). The ratio of rates of these reactions is insensitive to temperature so that the calculated ${ }^{12} \mathrm{C} /{ }^{13} \mathrm{C}$ ratio at any depth in a stellar model is very robust. The depth of mixing in the outer hydrogen convection zone increases as the star evolves to become a red giant, thereby bringing material with enhanced ${ }^{13} \mathrm{C}$ to the stellar surface. For small mass stars this occurs rather suddenly as the star leaves the subgiant branch and starts to evolve to higher luminosities.

The theory of mixing in the outer regions of stars is not simple. If we look at stellar models of cool stars from the outside inward we see that in the outermost layers, say with an optical depth less than unity, heat is transferred by radiation with most of the radiation escaping the star. For temperatures starting around 7000$8000 \mathrm{~K}$, photoionization of hydrogen from the first and second excited states causes the opacity to rise rapidly in the wavelength regions of maximum flux. This causes the temperature gradient, which is proportional to the opacity in a radiative atmosphere, to rise beyond the point of convective instability (Schwarzschild, 1906). So long as the radiative temperature gradient exceeds the limit set by 


$$
\frac{d \log T}{(d \log P)_{\mathrm{ad}}} \sim 0.4
$$

convection will take place. The fraction of energy carried by convection will depend on the density and the mixing length, defined as the distance over which a convective element travels before losing its identity. Theoretical estimates of the mixing length are difficult to calculate but we know enough about the sun, i.e., its mass, radius, luminosity, helium content, and vibration modes to establish a ratio of mixing length to scale height of $l / h \simeq 1.75$.

The application of the solar mixing length to red giants is questionable. The low density in a red giant's atmosphere means that convection will be less efficient. Hence radiation will still carry a substantial fraction of the flux and convective velocities will be higher than in the sun to compensate for the low density. Furthermore, the contribution to the opacity by bound-bound transitions in the sun in the $10^{5}-10^{6} \mathrm{~K}$ region (Rogers and Iglesias, 1992) depends on the metal abundance, which is reduced by a factor of as much as 200 in globular clusters and metal-poor fields stars, thus reducing their opacity and radiative temperature gradient. Hence the applicability of the solar ratio of $l / h$ to red giants of radius $10-50 R_{\odot}$ (and hence a surface gravity reduced by $10^{2}$ to $2.5 \times 10^{3}$ ) is questionable.

Convective overshooting at the bottom of the formal convection zone may be significant. The higher densities at those depths and positive density gradient tend to limit the overshooting to a fraction of a scale height. Overshooting is not sufficient to extend it to depths at which the ${ }^{12} \mathrm{C}$ once on the surface will be convected down to where it will be subjected to proton capture, i.e., where the temperature is more than about $1.5 \times 10^{7}$ $\mathrm{K}$.

The problem is how to mix material from the bottom of the convection zone to deeper layers. This problem is also of importance in the sun and other main-sequence stars of similar mass and age to the sun because the low Li abundance in the solar atmosphere cannot be explained by standard convection theory. Mixing must be induced by another effect. Two possibilities have been suggested. These are turbulence associated with a gradient in solar rotation and ionic diffusion (Richard et al., 1996).

\section{A. CN cycle and mixing}

A good example in which the models may be compared to theory is the rather old open cluster, M67. In Fig. 10 we show the results of calculations of the ${ }^{12} \mathrm{C} /{ }^{13} \mathrm{C}$ ratio for evolving stars in the theoretical HR diagram. The initial ratio was assumed to be 89 , the solar system value. The ratio drops rapidly as the star evolves off the subgiant branch and remains near 25 for its entire lifetime on the giant branch as well as on the "clump" of post-helium-flash models (Sweigert et al., 1989). Observations of the ${ }^{12} \mathrm{C} /{ }^{13} \mathrm{C}$ ratio by Gilroy and Brown (1991) are shown in Fig. 11 in the observational HR diagram.

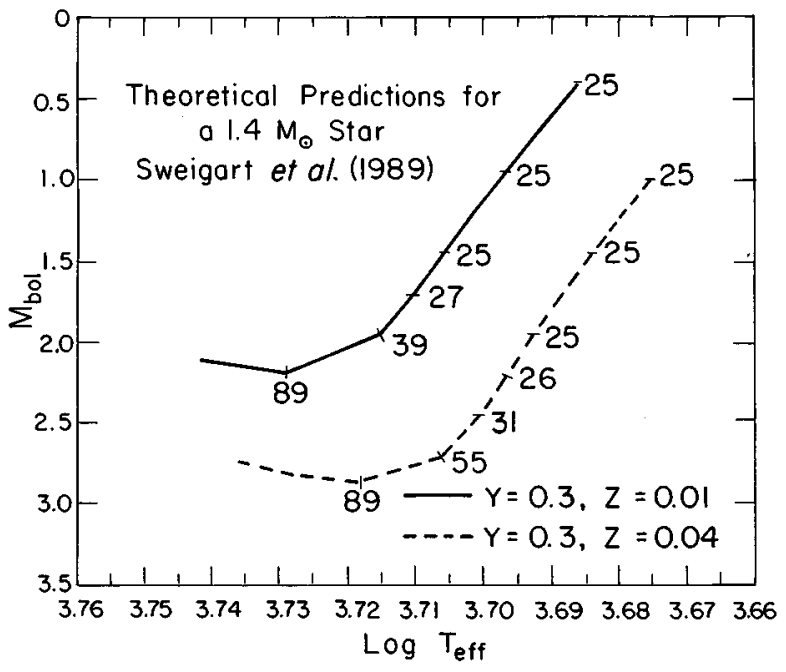

FIG. 10. Theoretical predictions of ${ }^{12} \mathrm{C} /{ }^{13} \mathrm{C}$ ratio for a $1.4 \mathrm{M} \odot$ star by Sweigert et al. (1989) for two different compositions. (Courtesy of the Astrophysical Journal.)

The real stars follow the models up the giant branch with just about the predicted ${ }^{12} \mathrm{C} /{ }^{13} \mathrm{C}$ ratio until they reach $T_{\text {eff }} \simeq 4200$ at which point additional mixing to greater depths further lowers the ${ }^{12} \mathrm{C} /{ }^{13} \mathrm{C}$ ratio to near 10. Models with standard convection theory fail to predict this additional mixing. Recent progress on the problem of mixing in small mass red giants and their ${ }^{12} \mathrm{C} /{ }^{13} \mathrm{C}$ ratios has been made by Charbonnel (1995) and by Boothroyd and Sackmann (1997).

The analysis of stars in many open clusters of differing ages and hence differing turn-off masses by Gilroy (1989) shows that the standard theory holds well for masses greater than about $2.0 M_{\odot}$ but fails to predict the lower ${ }^{12} \mathrm{C} /{ }^{13} \mathrm{C}$ ratios in the smaller mass stars. The extreme example is the metal-poor red giants analyzed by

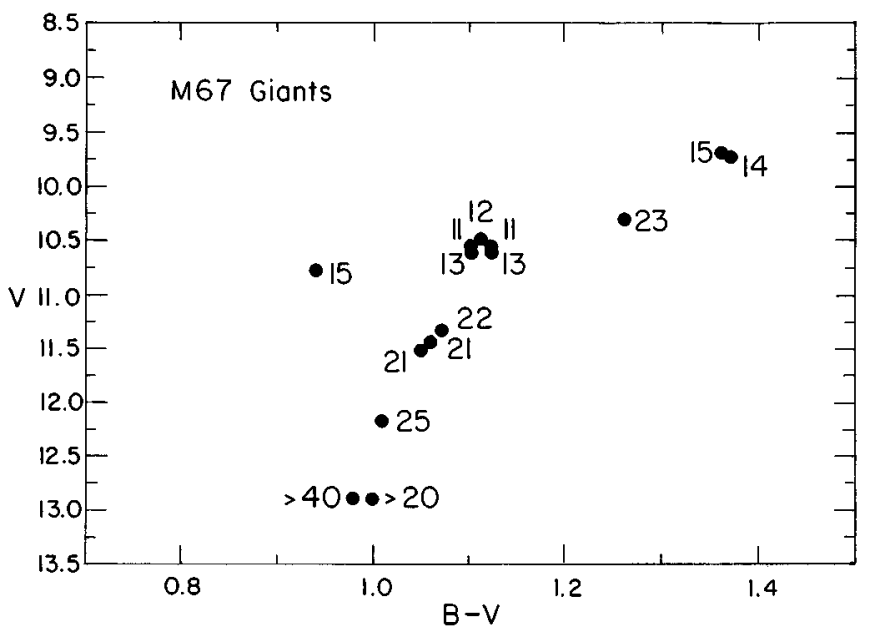

FIG. 11. Member stars of the cluster M67. The ${ }^{12} \mathrm{C} /{ }^{13} \mathrm{C}$ ratio for each star is shown beside the corresponding star. The color index, $\mathrm{B}-\mathrm{V}$, transforms to $\log T_{\text {eff }}$ with $\mathrm{B}-\mathrm{V}=1.0$ and 1.3 representing $\log T_{\text {eff }}=3.70$ and 3.65, respectively. (Courtesy of the Astrophysical Journal.) 
Sneden et al. (1986). Those stars, with metallicities similar to globular cluster stars, probably have masses near $0.8 M_{\odot}$. They show ${ }^{12} \mathrm{C} /{ }^{13} \mathrm{C}$ ratios near four, which is nearly the equilibrium abundance ratio under proton burning by the $\mathrm{CN}$ cycle. In globular clusters the ratio can be measured only for stars near the red giant tip of the color-magnitude diagram, where the ratio is found to lie in the range of 4 to 6 . Such small ratios indicate that not only has fresh ${ }^{13} \mathrm{C}$ been scooped up but that most of the visible material on the stellar surface has been carried down to burning depths so there has been almost no dilution of material processed at depth. Further evidence for depletion of ${ }^{12} \mathrm{C}$ by proton capture is provided by the analysis of giants from $M_{v}=+0.8$ to -2.2 in the globular cluster NGC6397 showing steady C depletion as stars evolve up the red giant branch (Briley et al., 1996).

\section{B. O depletion and the enhancement of $\mathrm{Na}$ and $\mathrm{Al}$}

Within the past few years proton captures by various isotopes from oxygen through magnesium have been invoked to explain the low oxygen combined with excess $\mathrm{Na}$ and $\mathrm{Al}$ in globular cluster red giants (Kraft, 1994; Kraft et al., 1997 and references therein). At the time of the writing of $\mathrm{B}^{2} \mathrm{FH}$ no member of a globular cluster had been analyzed in detail for its chemical composition. Multicolor photometry and low resolution spectroscopy had strongly suggested that globular cluster stars were metal deficient, probably similar to the subdwarfs analyzed by Chamberlain and Aller (1951). Using spectra obtained by Greenstein with the then new 200 inch telescope and exposures of up to three full nights, Helfer et al. (1959) found metal deficiencies of a factor of 20 and 200, as compared with the sun for stars in M13 and M92. Of the various elements studied no substantial discrepancies of their ratios to iron (as compared to the solar ratio) were found among the elements studied except for a deficiency of $\mathrm{Ba}$ and the rare earths in their M92 star whose $[\mathrm{Fe} / \mathrm{H}]$ value is -2.3 . Oxygen, sodium, and aluminum were not among the species for which useful data were available. High resolution spectroscopy of numerous globular cluster stars was not possible until the $4 \mathrm{~m}$ telescopes with their echelle spectrographs and image tube detectors became operational in the mid 1970 's. That combination made globular cluster stars accessible in the spectral region extending to about $8000 \AA$ at high resolution (but usually with lower signal to noise than the observers realized). By the mid 1980s CCD detectors replaced the image tubes and their photographic plates.

The first hint of unexpected proton captures by ${ }^{16} \mathrm{O}$ and heavier species was reported by Pilachowski et al. (1980) from the study of five red giants in the globular cluster M13, where a maximum difference of 1.5 dex in $[\mathrm{O} / \mathrm{Fe}]$ was reported for the most extreme stars in their sample. The large spread of $\mathrm{O} / \mathrm{Fe}$ in $\mathrm{M} 13$ was successively confirmed with improving accuracy by Leep et al. (1986), Hatzes (1987), and Brown et al. (1991). In definitive studies Kraft et al. (1993; also Kraft et al., 1997) found a spread from $[\mathrm{O} / \mathrm{Fe}]=+0.46$ to -0.71 among the red giants in M13. They attributed the O-poor stars to ON cycling and mixing of processed matter to the surface.

The evidence for $\mathrm{Na}$ enhancements in the O-poor star, II-67, in M13 also dates from 1980 (Peterson, 1980). She found $[\mathrm{Na} / \mathrm{Fe}]=+0.69$ in the O-poor star II-67 while $[\mathrm{Na} / \mathrm{Fe}]=-0.34$ and +0.13 in two O-normal stars. A followup large scale study by Kraft et al. (1993) showed a clear correlation of $\mathrm{Na}$ excess with $\mathrm{O}$ deficiency in many stars in M13 and a few stars in M3.

In M10, a cluster with a very blue horizontal branch, like M13, Kraft et al. (1995) have found $[\mathrm{O} / \mathrm{Fe}]$ to range from +0.5 to -0.35 in an analysis of 14 stars. For six stars with $[\mathrm{O} / \mathrm{Fe}]>\mathrm{O},[\mathrm{Na} / \mathrm{Fe}]=-0.65 \pm 0.14$, while for seven stars with $[\mathrm{O} / \mathrm{Fe}]<\mathrm{O},[\mathrm{Na} / \mathrm{Fe}]=0.23 \pm 0.08$. A variety of other species from $\mathrm{Si}$ to $\mathrm{Ni}$ show no correlation with $[\mathrm{O} / \mathrm{Fe}]$. Aluminum lines were not observed. In none of these clusters was a significant spread in $[\mathrm{Fe} / \mathrm{H}]$ detectable.

Perhaps the most interesting database is that of Norris and Da Costa (1995, NDC) for Omega Centauri (see also Zucker et al., 1996), the most massive globular cluster in our Galaxy. Omega Cen is the only globular to show a wide spread in metallicity, certainly due to selfenrichment. NDC analyzed 40 red giants with a range in $[\mathrm{Fe} / \mathrm{H}]$ from -1.9 to -0.8 . For species such as $\mathrm{Si}, \mathrm{Ca}$, and Ti the spread in $[\mathrm{X} / \mathrm{Fe}]$ is no more than might be expected from observation and analytical uncertainties and no trend with $[\mathrm{Fe} / \mathrm{H}]$ is discernible. The same holds for four iron-peak elements. The $[\mathrm{O} / \mathrm{Fe}]$ value ranges from +0.5 (as commonly seen in metal-poor stars) down to $<-0.4$ throughout the entire range of $[\mathrm{Fe} / \mathrm{H}]$. Most stars are depleted in carbon and enhanced in nitrogen, as is expected by $\mathrm{CN}$ and $\mathrm{CNO}$ cycling. The sum of $\mathrm{C}+\mathrm{N}+\mathrm{O}$ appears to be constant, near $+0.3 \mathrm{dex}$, as is usually the case for metal-poor stars. Of course this points to a rearrangement of the $\mathrm{CNO}$ nuclei during proton captures. There are no trends with $[\mathrm{Fe} / \mathrm{H}]$ to indicate that the cause of the changes is related to the general, and presumably gradual, metallicity enhancement in the cluster.

When the abundances of $\mathrm{Na}, \mathrm{Mg}$, and $\mathrm{Al}$ are examined the plot thickens. For $\mathrm{Na}$ the correlation of $[\mathrm{Na} / \mathrm{Fe}]$ with $[\mathrm{O} / \mathrm{Fe}]$ is similar to that in M13, running from $[\mathrm{Na} /$ $\mathrm{Fe}] \approx+0.6$ for stars with $[\mathrm{O} / \mathrm{Fe}]$ between -0.1 and $<-0.4$ to $[\mathrm{Na} / \mathrm{Fe}]$ ranging from -0.3 to +0.3 for the stars with $[\mathrm{O} / \mathrm{Fe}]$ between 0.0 and 0.6. For aluminum the range is larger. The $\mathrm{O}$-poor stars show $[\mathrm{Al} / \mathrm{Fe}]$ as large as +1.0 while the $\mathrm{O}$-normal stars show $[\mathrm{Al} / \mathrm{Fe}]=+0.4$ to about -0.2 , with seven $\mathrm{Al}$ abundances being only upper limits. These trends were confirmed and strengthened by Zucker et al. (1996) who observed some additional Al lines not covered by NDC. In Omega Cen the correlation of $\mathrm{Mg}$ and $\mathrm{Al}$ is really strange. There is a clear break in $[\mathrm{Al} / \mathrm{Fe}]$ at +0.5 dex. The low-Al stars range from $[\mathrm{Al} / \mathrm{Fe}]=+0.4$ to $\lesssim-0.2$. All of them show $[\mathrm{Mg} / \mathrm{Fe}] \simeq+0.45$ which is normal for metal-poor stars. The Al-rich stars with $[\mathrm{Al} / \mathrm{Fe}]=+0.7$ to +1.1 show a range of $[\mathrm{Mg} / \mathrm{Fe}]$ values from +0.5 to -0.1 with no correlation 
TABLE IV. Abundances on the $\log N$ scale with $\log N(H)=12$ for O-rich (i.e., normal) and O-poor (i.e., depleted) stars in M13 and Omega Centauri.

\begin{tabular}{lccccc}
\hline \hline & \multicolumn{5}{c}{$\log N$} \\
\hline Object & ${ }^{16} \mathrm{O}$ & ${ }^{20} \mathrm{Ne}$ & ${ }^{23} \mathrm{Na}$ & ${ }^{24} \mathrm{Mg}$ & ${ }^{27} \mathrm{Al}$ \\
Sun $^{\mathrm{a}}$ & 8.9 & $8.1:$ & 6.3 & 7.6 & 6.5 \\
M13, O-rich $^{\mathrm{b}}$ & 7.6 & $6.8:$ & 4.5 & 6.3 & 4.9 \\
M13, O-poor $^{\mathrm{b}}$ & 6.5 & $?$ & 5.1 & 6.0 & 6.1 \\
Omega Cen, O-rich $^{\mathrm{c}}$ & 7.6 & $6.8:$ & 4.6 & 6.4 & 4.9 \\
Omega Cen, O-poor $^{\mathrm{c}}$ & 6.9 & $?$ & 5.5 & 6.4 & 5.8 \\
Omega Cen, O- and Mg-poor $^{\mathrm{c}}$ & 6.8 & $?$ & 5.2 & 6.0 & 5.8 \\
\hline
\end{tabular}

${ }^{\mathrm{a}}$ From Anders and Grevesse (1989).

${ }^{\mathrm{b}}$ Assuming $[\mathrm{Fe} / \mathrm{H}]=-1.6$.

${ }^{\mathrm{c}}$ Adjusted to $[\mathrm{Fe} / \mathrm{H}]=-1.6$ using individual $[\mathrm{Fe} / \mathrm{H}]$ values from NDC.

with $[\mathrm{Al} / \mathrm{Fe}]$. If $\mathrm{Al}$ is produced by $p$ captures on ${ }^{24} \mathrm{Mg}$ (as will be discussed below) this distribution is very strange because it indicates that the $\mathrm{Mg}$ abundance is unchanged as the $\mathrm{Al}$ abundance increase by a factor of 10 , except in five stars in which $\mathrm{Mg}$ was depleted by a factor 3 as $\mathrm{Al}$ increased by the same amount as it did in the Mg-normal stars. The Mg-poor stars show moderate metal deficiencies with $[\mathrm{Fe} / \mathrm{H}]$ ranging from -1.67 to $-1.35,[\mathrm{Na} / \mathrm{Fe}]$ excesses near +0.5 and large oxygen depletions. One problem is that there is no really secure baseline for $[\mathrm{Al} / \mathrm{Fe}]$ which can be described as "normal" for a metal-poor globular. The Mg-deficient stars are some of the brightest red giants in Omega Cen. In moderately metal-poor main sequence stars, the $[\mathrm{Al} / \mathrm{Fe}] \mathrm{ra}-$ tio is slightly positive, about +0.2 dex, but ranging from 0.0 to +0.4 (Edvardsson et al., 1993). For dwarfs with $[\mathrm{Fe} / \mathrm{H}]$ between -1 and $-2,[\mathrm{Al} / \mathrm{Fe}]$ is about +0.1 (Francois, 1986).

In M13 there are similar correlations (Kraft et al., 1997, and references therein). As $[\mathrm{O} / \mathrm{Fe}]$ descends from +0.4 to $-0.2,[\mathrm{Na} / \mathrm{Fe}]$ rises from -0.2 to +0.4 and remains near +0.4 for the most $\mathrm{O}$-poor stars at $[\mathrm{O} / \mathrm{Fe}]=$ -0.8 . Metal-poor field giants do not show a significant spread in $[\mathrm{O} / \mathrm{Fe}]$ but show the usual $[\mathrm{O} / \mathrm{Fe}] \simeq 0.3$, though $\mathrm{Na}$ ranges from $[\mathrm{Na} / \mathrm{Fe}]=-0.6$ to 0 . In M13 the ratio of $\mathrm{Al} / \mathrm{Fe}$ shows a tighter correlation with oxygen in the same sense as does $\mathrm{Na}$ with $[\mathrm{Al} / \mathrm{Fe}]$ ranging from 0.0 at $[\mathrm{O} / \mathrm{Fe}]=+0.4$ to +1.2 for $[\mathrm{O} / \mathrm{Fe}]$ between -0.2 and -0.8 . Magnesium shows the standard excess of $[\mathrm{Mg} / \mathrm{Fe}]=+0.3$ for $0.4 \geq[\mathrm{O} / \mathrm{Fe}] \geq-0.1$ and then drops to 0.0 for $[\mathrm{O} / \mathrm{Fe}]$ $\leq-0.2$. At this point it is best to revert to absolute abundances. In Table IV we show the logarithmic abundances of the critical species, $\mathrm{O}, \mathrm{Na}, \mathrm{Mg}$, and $\mathrm{Al}$ on the usual arbitrary astronomical scale of $\log N(H)=12$.

The oxygen-rich stars show abundances similar to metal-poor field stars and similar to metal-poor stars in many other globular clusters. Hence the O-poor stars are the abnormal ones.

\section{Origin of the $\mathrm{Na}$ and $\mathrm{Al}$ enhancements}

If the excesses of $\mathrm{Na}$ and $\mathrm{Al}$ seen in the O-poor stars are due to proton captures and subsequent mixing within the observed stars themselves, Table IV tells us what must have happened. First we must look at the nuclear physics and secondly we will look at the problem of mixing. In 1957 Marion and Fowler suggested that under the right conditions a $\mathrm{NeNa}$ cycle could occur by successive proton captures by the $\mathrm{Ne}$ isotopes followed by $\beta$ decays and ending with ${ }^{23} \mathrm{Na}(p, \alpha){ }^{20} \mathrm{Ne}$. The analogy to the $\mathrm{CN}$ cycle is obvious. The $\mathrm{NeNa}$ cycle is not likely ever to be a major energy source but it can readily convert significant amounts of ${ }^{22} \mathrm{Ne}$ into ${ }^{23} \mathrm{Na}$ (provided that the star's original ${ }^{22} \mathrm{Ne} /{ }^{23} \mathrm{Na}$ ratio was similar to the solar ratio of 4.1 ) or ${ }^{20} \mathrm{Ne}$ to ${ }^{23} \mathrm{Na}$ by three $p$ captures and a sufficiently low temperature or low cross section for $p$ capture by ${ }^{23} \mathrm{Na}$. Denissenkov and Denissenkova (1990) were the first to suggest that the NeNa cycle may be effective in cool stars. There have been many follow up reviews such as Briley et al. (1994), Kraft (1994), and Kraft et al. (1997), the first concentrating on CNO and the latter two on the Na-Mg-Al problem.

The tough question is the production of the excess Al. In M13, $\mathrm{Al}$ must rise from $\log N=4.9$ to $\log N=6.1$, while in Omega Cen the rise is from 4.9 to 5.8. If the source is ${ }^{24} \mathrm{Mg}$, approximately half of the original ${ }^{24} \mathrm{Mg}$ must have been converted to ${ }^{27} \mathrm{Al}$ in M13, leaving the other half as $\mathrm{Mg}$ in accordance with the changes shown in Table IV. This solution excludes any dilution by unprocessed material which would reduce the surface $\mathrm{Al}$ abundance and restore the surface $\mathrm{Mg}$ toward its original value. For Omega Cen the rise in $\mathrm{Al}$ must be from $\log N=4.9$ to 5.8 according to NDC and to 6.0 according to Zucker et al. who measured four lines, rather than one or two by NDC. In that case, a third of the original ${ }^{24} \mathrm{Mg}$ must have been processed. If the $\mathrm{Mg}$-poor stars in Omega Cen really are depleted from $\log N=6.4$ to 6.0 , then conservation of nuclei implies that their $\mathrm{Al}$ abundance must be $\log N=6.2$. These severe requirements strain credulity. To overcome them, Cavallo et al. (1996) suggest that ${ }^{20} \mathrm{Ne}$, which presumably is present with $\log N=6.9$ (assuming its ratio to iron is enhanced, as are $\mathrm{O}$ and $\mathrm{Mg}$, in most globular cluster stars), may be processed up to Al. The trouble with their solution is the tendency for the $\mathrm{NeNa}$ sequence to cycle because the reaction rate of ${ }^{23} \mathrm{Na}(p, \alpha)$ is larger than the rate for ${ }^{23} \mathrm{Na}(p, \gamma)$. The rates are fully discussed in Sec. VII of this review by A. Champagne. The most successful effort to date to explain the excess of $\mathrm{Al}$ (Cavallo et al., 1997) requires that ${ }^{24} \mathrm{Mg}$ be the source of the $\mathrm{Al}$ excess. For M13, as shown in Table IV, the ratio of ${ }^{24} \mathrm{Mg}$ in O-rich stars to ${ }^{27} \mathrm{Al}$ in $\mathrm{O}$-poor stars is only 0.2 dex. Hence much of the original ${ }^{24} \mathrm{Mg}$ must be processed to ${ }^{27} \mathrm{Al}$; it must not cycle back to ${ }^{24} \mathrm{Mg}$ and must not capture an additional proton to produce ${ }^{28} \mathrm{Si}$.

If the observed stars did not enhance their $\mathrm{Al}$ (and perhaps the $\mathrm{Na}$ as well), then their observed abundances must have been inherited from inhomgeneous enrichment very early in the lifetime of the cluster as suggested by Cottrell and Da Costa (1981) for 47 Tuc and NGC 6752. Additional evidence for an inhomogeneity of $\mathrm{Na}$ has been presented by Briley et al. (1996) for the cluster 47 Tucanae, the second most massive globular in 
our Galaxy. They have measured the sodium D lines in six stars near the turnoff of the main sequence where convection cannot possibly mix material from the center to the surface and where central temperatures are near $2 \times 10^{7} \mathrm{~K}$, too cool to run the $\mathrm{NeNa}$ cycle. The derived $\mathrm{Na}$ abundances run from $\log N=5.4$ to 5.8 , while the derived $\mathrm{Ca}$ abundances show no correlation with the $\mathrm{Na}$ abundances. Furthermore the $\mathrm{Na}$ abundances correlate with the strength of the $\mathrm{CN}$ band at $3883 \AA$ showing a correlation of $\mathrm{Na}$ with the $\mathrm{N}$ abundance. Current models have so far been unable to explain this quantitatively, so either the models are seriously in error or the $\mathrm{Na}$ and $\mathrm{CN}$ excesses were present at the time of star formation. Detailed calculations by Denissenkov et al. (1997) show that metal-poor AGB stars of 5-8 $M_{\odot}$ are able to produce (and presumably eject by a stellar wind) an excess of ${ }^{23} \mathrm{Na}$ but fail to produce the observed excess of ${ }^{27} \mathrm{Al}$.

Self-enrichment by globular clusters during their early years has been shown to be very likely (Morgan and Lake, 1989). With much interstellar matter still in the cluster, gas and dust ejected by massive stars may be confined within a small fractional volume of the cluster. After the intracluster matter has been blown away, probably by supernova explosions, further ejecta from stars will easily escape from the relatively weak gravitational well of the cluster. Massive stars are known to lose material at a prodigeous rate, especially as WolfRayet (WR) stars. The latter type show an excess of N, $\mathrm{C}, \mathrm{O}$, and presumably ${ }^{22} \mathrm{Ne}$ in their atmospheres. Lines of $\mathrm{Na}$ are not likely to be present in WR stars but the Al III resonance lines at 1855 and $1863 \AA$ were readily observable with the International Ultraviolet Explorer and may easily be observed with the Hubble Space Telescope. Their analysis to derive the $\mathrm{Al}$ abundance in WR stellar winds would be extremely valuable. The same holds for the analysis of other hot stars with strong nitrogen lines known as OBN stars (Walborn, 1987).

Clearly we are seeing proton captures by ${ }^{16} \mathrm{O}$, and by the Ne isotopes as first suggested by Marion and Fowler $(1957)^{2}$ to enhance $\mathrm{Na}$ and by the $\mathrm{Mg}$ isotopes to produce the excess Al. The uncertain question is whether the observed star is responsible for polluting its own atmosphere or did the star inherit its unusual composition while still forming from a nearby rapidly evolving neighbor? For ${ }^{16} \mathrm{O}$ there is no question but that $\mathrm{CNO}$ cycling is responsible because the sum of $\mathrm{C}+\mathrm{N}+\mathrm{O}$ is the same in the O-rich and O-poor stars in M13 (Brown et al., 1991); however, that does not tell us where or when it happened.

\section{CARBON, NEON, OXYGEN, AND SILICON BURNING}

\section{A. Introduction}

In addition to producing ${ }^{12} \mathrm{C}$ and ${ }^{16} \mathrm{O}$, helium burning was identified by $\mathrm{B}^{2} \mathrm{FH}$ as the source of ${ }^{20} \mathrm{Ne}$ and pos-

\footnotetext{
${ }^{2}$ The possibility of the reaction ${ }^{22} \mathrm{Ne}(p, \gamma){ }^{23} \mathrm{Na}$ was noticed by Bethe (1939, Table VI).
}

sibly some ${ }^{24} \mathrm{Mg}$. It is now known that there is no suitable excited state of ${ }^{20} \mathrm{Ne}$ near the $\alpha+{ }^{16} \mathrm{O}$ threshold to serve as a resonance for $\alpha$ capture on ${ }^{16} \mathrm{O}$. (See Sec. VI of this review.) Formation of nuclei heavier than ${ }^{16} \mathrm{O}$ then proceeds successively by core carbon, neon, oxygen, and silicon burning. (Of these processes, only neon burning was included in $\mathrm{B}^{2} \mathrm{FH}$.)

An important new feature of the carbon burning and ensuing stages is that the star's dominant luminosity (i.e., its energy loss) is by neutrino emission directly from the core of the star, rather than electromagnetic radiation from the star's surface. A natural result of this prodigious luminosity is that the star's core temperature is increased, evolution is greatly sped up, and carbon burning and the following stages have successively shorter time durations.

The exhaustion of carbon in the core is followed at $\mathrm{T}_{9} \sim 1.3$ by a process called neon burning, in which the ambient blackbody radiation photodisintegrates the matter present with the lowest $\alpha$ threshold energy, ${ }^{20} \mathrm{Ne}$, with a $(\gamma, \alpha)$ threshold of only $4.7 \mathrm{MeV}$. The $\alpha$ particles liberated in this way can react with ${ }^{20} \mathrm{Ne}$ to form ${ }^{24} \mathrm{Mg}$. Although the photodisintegration is endoergic, the exoergic reaction that forms ${ }^{24} \mathrm{Mg}$ has a higher energy release ( $Q$ value), and the overall combination of the two reactions is exoergic.

Following neon burning, the core contracts and heats to $\mathrm{T}_{9} \sim 2$ when oxygen can begin to burn. The principal immediate products are ${ }^{28} \mathrm{Si},{ }^{31} \mathrm{P}$, and ${ }^{31} \mathrm{~S}$, but a large range of nuclei are also built up to $A \sim 40$, with the $A$ $=4 n$ nuclei favored by their higher binding energies. Together, neon burning and oxygen burning are the modern version of the $\alpha$ process of $\mathrm{B}^{2} \mathrm{FH}$.

The temperature increases throughout oxygen burning and eventually, at $\mathrm{T}_{9} \sim 3-3.5$, oxygen burning merges into silicon burning, which can best be described as the photodisintegration of nuclei to produce $\alpha$ particles, protons, and neutrons from the nuclei present, beginning with those with the lowest thresholds, and finally consuming the most "refractory" nuclei like ${ }^{28} \mathrm{Si}$. The freed alphas, protons, and neutrons build nuclei up to the region of the most stable nuclei, the iron abundance peak. If the buildup occurs rapidly, with little increase of the neutron excess, $\eta=\sum_{i}\left(N_{i}-Z_{i}\right) / A_{i}$, the most prominent nucleus will be ${ }^{56} \mathrm{Ni}$. On the other hand, if the buildup occurs slowly, $\eta$ will increase during silicon burning from its initial value of about $1.5 \times 10^{-3}$ to $10^{-2}$ or even higher, and the most abundant nucleus will shift to ${ }^{54} \mathrm{Fe},{ }^{56} \mathrm{Fe}$, or ${ }^{58} \mathrm{Fe}$ for larger $\eta$ (see Sec. XIV of this review).

The observation in $\gamma$ detectors carried by both satellites and high altitude balloons of $\gamma$ rays from SN1987a, which arise from the positron decays ${ }^{56} \mathrm{Ni} \rightarrow{ }^{56} \mathrm{Co}+e^{+}+\nu_{e}, \quad{ }^{56} \mathrm{Co} \rightarrow{ }^{56} \mathrm{Fe}+e^{+}+\nu_{e}, \quad$ and ${ }^{57} \mathrm{Co} \rightarrow{ }^{57} \mathrm{Fe}+e^{+}+\nu_{e}$, confirms the general correctness of this picture (Matz et al., 1988; Rester et al., 1988; Mahoney et al., 1988; Teegarden et al., 1989; Sandie, et al., 1988).

A more detailed discussion of the scenarios for the various burning processes than space allows would dis- 
tinguish between core burning and shell burning, and also whether the burning is hydrostatic or explosive. In the case of explosive nucleosynthesis and the comparison of its products with the spectrum of radiation from stellar surfaces, or with solar system matter, such as terrestrial material and meteorites, it is always necessary to remember that much of the stellar material that has evolved through the most advanced explosive evolutionary stages may remain "locked up" in some form of supernova remnant, such as a neutron star or black hole. Other matter that may not have gone through advanced evolution may be locked up in low mass white or brown dwarfs.

\section{B. Carbon burning}

The suggestion that carbon burning would occur after helium burning was made by Salpeter (1952). For carbon burning, the "Gamow Window" peaks at $E_{0}=2.42$ $T_{9}^{2 / 3} \mathrm{MeV}$ and has a width $\Delta=1.054 T_{9}^{5 / 6}$; from stellar models the temperature range for hydrostatic carbon burning is $0.8 \leq T_{9} \leq 1.2$. The principle reactions are

$$
\begin{aligned}
{ }^{12} \mathrm{C}+{ }^{12} \mathrm{C} & \rightarrow{ }^{20} \mathrm{Ne}+{ }^{4} \mathrm{He}+4.617 \mathrm{MeV} \\
& \rightarrow{ }^{23} \mathrm{Na}+p+2.241 \mathrm{MeV} \\
& \rightarrow{ }^{23} \mathrm{Mg}+n-2.599 \mathrm{MeV} .
\end{aligned}
$$

Clearly, the neutron producing reaction will be the weakest of these, especially at lower temperatures. Other ${ }^{12} \mathrm{C}+{ }^{12} \mathrm{C}$ reactions are possible but are believed to be much less important for a variety of reasons. The freed $\alpha$ particles, protons, and neutrons can interact with the nuclei present, including CNO nuclei from hydrogen and helium burning, to build a range of nuclei including ${ }^{20,21,22} \mathrm{Ne},{ }^{23} \mathrm{Na},{ }^{24,25,26} \mathrm{Mg},{ }^{26,27} \mathrm{Al},{ }^{28,29,30} \mathrm{Si}$, and ${ }^{31} \mathrm{P}$, but the main products are ${ }^{20} \mathrm{Ne}$ and ${ }^{24} \mathrm{Mg}$, the latter from $\alpha$ capture on ${ }^{20} \mathrm{Ne}$, or ${ }^{23} \mathrm{Na}(p, \gamma)$.

After early studies at the Chalk River Laboratories (Almqvist et al., 1960), ${ }^{12} \mathrm{C}+{ }^{12} \mathrm{C}$ studies were resumed at Caltech at lower energies (Patterson, Winkler, and Zaidins, 1969). By this time it was known that a state in ${ }^{20} \mathrm{Ne}$ that had appeared to be a possible resonance for radiative $\alpha$ capture by ${ }^{16} \mathrm{O}$ had a combination of $J$ and parity that cannot be formed by ${ }^{16} \mathrm{O}+{ }^{4} \mathrm{He}$, and that formation of ${ }^{20} \mathrm{Ne}$ by helium burning would not proceed without a much higher temperature than helium burning would provide. It was then realized that carbon burning would follow the production of ${ }^{12} \mathrm{C}$ and ${ }^{16} \mathrm{O}$ in helium burning.

Numerous other studies of the ${ }^{12} \mathrm{C}+{ }^{12} \mathrm{C}$ reactions have since been published (Dayras et al., 1977; Mazarakis and Stephens, 1972; High and Cujec, 1977; Kettner et al., 1980; Erb et al., 1980; Becker et al. 1981). The most striking revelation of these studies is that the yield vs energy curve ("excitation function") displays a strongly resonant structure, the origin of which remains obscure, notwithstanding many suggestions regarding this intermediate structure (see Fig. 12). The various experiments also reveal rather poor agreement at the lowest energies,

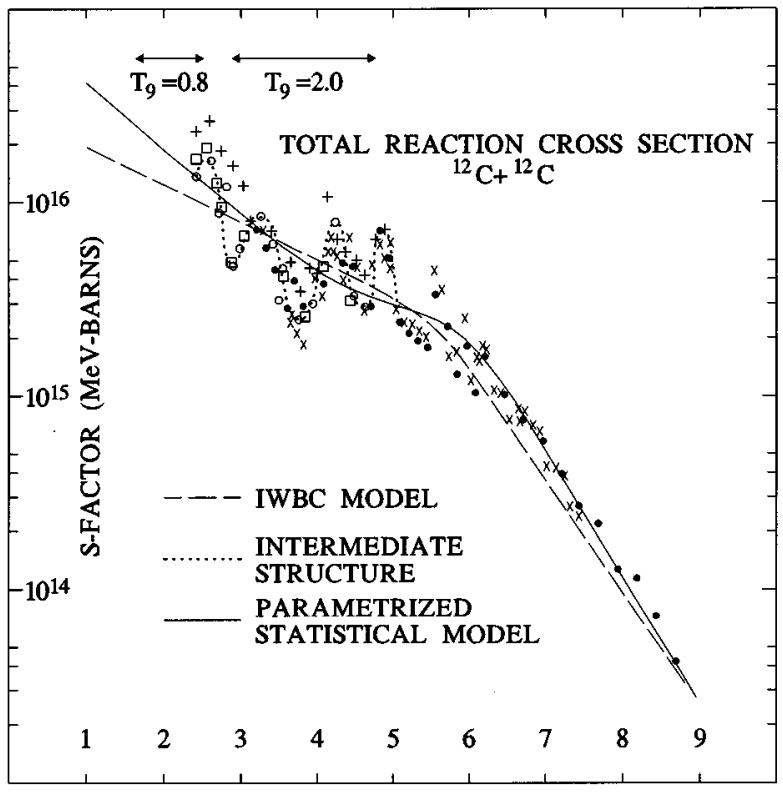

CM ENERGY (MeV)

FIG. 12. ${ }^{12} \mathrm{C}+{ }^{12} \mathrm{C} \mathrm{S}$ factor vs center-of-mass energy. The data shown are from Patterson et al. (1969), black dots and $\times$ 's; Mazarakis and Stephens (1972), plus signs; High and Cujec (1977), open squares; and Kettner et al. (1980), open circles. The dotted curve is a rough sketch of the intermediate structure resonances. The dashed curve is an incoming wave boundary condition model, and the solid curve is a statistical model fit of Caughlan and Fowler (1988).

and even the trend of the $\sigma(E)$ curve, or the $S(E)$ curve, remains somewhat uncertain at the lower energies. The results of Mazarakis and Stephens led Michaud (1972) to postulate a new phenomenon called "penetration under the barrier," attributable to a nucleusnucleus potential that had its imaginary (absorptive) part extending to radii larger than the real part of the potential. This was assumed in order to produce an enhancement of the cross-section curve above the conventional barrier penetration expectation because it would effectively result in a thinner Coulomb barrier. However, later studies of ${ }^{12} \mathrm{C}+{ }^{12} \mathrm{C}$ and other heavy-ion reactions such as ${ }^{12} \mathrm{C}+{ }^{16} \mathrm{O}$ (Cujec and Barnes, 1976) did not support such low-energy enhancements. It seems possible that the observed effect was produced by a slightly erroneous effective beam energy, as the $\sigma(E)$ curve is a very steep function of $E$ at low energies. A review of the ${ }^{12} \mathrm{C}+{ }^{12} \mathrm{C}$ (and other heavy-ion reactions) has been published by Barnes et al. (1985); the average trend of the data (ignoring the resonances) has been fitted with a variety of optical models that give somewhat different values of the extrapolated cross sections. Fortunately, the measured $S(E)$ values cover the range $E_{0} \pm \Delta / 2$ for explosive carbon burning, and reach almost down to $E_{0}$ for hydrostatic carbon burning.

The ${ }^{12} \mathrm{C}+{ }^{16} \mathrm{O}$ reactions show some hints of resonant structure at the lowest energies. These reactions only become significant near ${ }^{12} \mathrm{C}$ exhaustion, and thus play only a small role, but they do help to improve agreement between predicted and measured abundances. 


\section{Neon burning}

At the end of carbon burning, at $\mathrm{T}_{9} \sim 1.2$, the remaining core nuclei are mainly ${ }^{16} \mathrm{O},{ }^{20} \mathrm{Ne}$, and ${ }^{24} \mathrm{Mg}$. Before oxygen can react with oxygen, the temperature becomes high enough to photodisintegrate ${ }^{20} \mathrm{Ne}$, because of its unusually low $\alpha$ threshold energy (4.73 MeV). The freed $\alpha$ particles can combine readily with ${ }^{20} \mathrm{Ne}$ to form ${ }^{24} \mathrm{Mg}$. Thus the reactions involved are

$$
{ }^{20} \mathrm{Ne}+\gamma \rightarrow{ }^{16} \mathrm{O}+{ }^{4} \mathrm{He}, \quad{ }^{20} \mathrm{Ne}+{ }^{4} \mathrm{He} \rightarrow{ }^{24} \mathrm{Mg}+\gamma
$$

and are equivalent, in an energetic sense, to

$$
{ }^{20} \mathrm{Ne}+{ }^{20} \mathrm{Ne} \rightarrow{ }^{16} \mathrm{O}+{ }^{24} \mathrm{Mg} \text {. }
$$

The overall products are rather similar to those from carbon burning, with the obvious exception that neon burning destroys neon, whereas carbon burning produces neon.

\section{Oxygen burning}

After neon burning has destroyed most of the core neon, the temperature rises to $\mathrm{T}_{9} \sim 2$, where oxygen can begin to fuse with oxygen. For oxygen burning, $E_{0}=3.91$ $T_{9}^{2 / 3} \mathrm{MeV}$ and $\Delta=1.34 T_{9}^{5 / 6} \mathrm{MeV}$. The principal reactions are

$$
\begin{aligned}
{ }^{16} \mathrm{O}+{ }^{16} \mathrm{O} & \rightarrow{ }^{28} \mathrm{Si}+{ }^{4} \mathrm{He}+9.594 \mathrm{MeV} \\
& \rightarrow{ }^{31} \mathrm{P}+p+7.678 \\
& \rightarrow{ }^{31} \mathrm{~S}+n+1.500 \\
& \rightarrow{ }^{30} \mathrm{Si}+2 p+0.381 \\
& \rightarrow{ }^{30} \mathrm{P}+d-2.409
\end{aligned}
$$

as well as several other branches with less favorable $Q$ values. The released $\alpha$ particles, protons, and neutrons interact with the nuclei present to produce a large spread of nuclei including ${ }^{28} \mathrm{Si},{ }^{32,33,34} \mathrm{Si},{ }^{35,37} \mathrm{Cl},{ }^{36,38} \mathrm{Ar}$, ${ }^{39,41} \mathrm{~K},{ }^{40,42} \mathrm{Ca},{ }^{46} \mathrm{Ti}$, and ${ }^{50} \mathrm{Cr}$, of which ${ }^{28} \mathrm{Si}$ and ${ }^{32} \mathrm{~S}$ are the main results (Woosley et al., 1972, 1973; Woosley, 1986; Arnett, 1996). The calculation of Arnett (1996) indicates that the neutron excess, $\eta$, increases from about $2 \times 10^{-3}$ to $\sim 6 \times 10^{-3}$ during core oxygen burning. Since such a large value of $\eta$ would give a nucleosynthetic result that disagrees with observation, it seems likely that the products of such core oxygen burning must be only rarely, if ever, ejected into the interstellar medium (ISM). Oxygen shell burning can proceed at lower density and higher temperature, up to $\mathrm{T}_{9} \sim 2.6-3$, and yields a distribution in better accord with solar system abundances (Arnett, 1996).

The burning process is accompanied by quasiequilibrium nuclear clusters that link together at higher temperatures; at the end of oxygen burning the nuclei form a cluster from ${ }^{24} \mathrm{Mg}$ to the mass region $40 \lesssim A \lesssim 50$, where the closed nuclear shells at $Z=20$ and $N=20$ and 28 inhibit propagation of the cluster to the iron-peak nuclei (Woosley, 1986; Arnett, 1996).

The curve of $S(E)$ vs $E$ for the ${ }^{16} \mathrm{O}+{ }^{16} \mathrm{O}$ reactions does not exhibit the resonant structure seen in ${ }^{12} \mathrm{C}+{ }^{12} \mathrm{C}$.
However, the various data sets do not agree well with one another at the lowest energies (Spinka and Winkler, 1972; Hulke et al., 1980; Wu and Barnes, 1984; Thomas et al., 1986). From the point of view of explosive oxygen burning where $\mathrm{T}_{9} \sim 3.1-3.9$, the experimental data cover most of the needed region of energies. For hydrostatic oxygen burning, at $T_{9} \sim 2$, less than half the needed energy region is covered by experiment, but the covered region includes the larger cross-section portion. Among the many model fits that have been made, we cite the time-dependent Hartree-Fock model calculations by Reinhard et al. (1984) and the parametrized statistical model fit of Caughlan and Fowler (1988). These fits agree within a factor of 2 over the energy range covered by experiment as well as the range of energies needed for both explosive and hydrostatic oxygen burning. Although this is reassuring, the experiments disagree among themselves by more than a factor of 5 at the lowest energies, and exhibit different trends at these energies [see, for example, Barnes et al. (1985)]. It is understandable that the different experimental methods pursued may suffer from different systematic errors, but a substantial improvement in the reproducibility of the low-energy data is clearly desirable.

\section{E. Silicon burning}

The silicon-burning process refers to the large group of nuclear reactions that start with the products of the previous burning processes and build the nuclei up to the iron peak in the abundance curve. Photodisintegration of the nuclei present plays a crucial role as it liberates the $\alpha$ particles, protons, and neutrons needed to carry out this buildup, first photodisintegrating the nuclei with the lowest thresholds and proceeding to the most refractory nuclei. An oversimplified but instructive picture of silicon burning would be to consider the process of breaking down ${ }^{28} \mathrm{Si}$ by $(\gamma, \alpha),(\gamma, p)$, and $(\gamma, n)$ processes, and building heavier nuclei by adding these particles in reactions such as $(\alpha, \gamma),(p, \gamma),(n, \gamma)$, $(\alpha, p)$, and $(\alpha, n)$ (Truran et al., 1966; Bodansky et al., 1968; Clayton, 1968). Core silicon burning begins at about $T_{9}=2.7$, allowing time for the neutron excess per nucleon, $\eta$, to increase while the temperature rises to about $T_{9}=4$ or higher. When the buildup reaches the situation where most of the core has reached the iron peak, the most abundant nuclei are likely to be ${ }^{54} \mathrm{Fe}$, ${ }^{56} \mathrm{Fe}$, and ${ }^{58} \mathrm{Ni}$. Here we see the residual effect of the $e$ process of $\mathrm{B}^{2} \mathrm{FH}$. However, not only would this produce too much iron-peak material if ejected into space, it is also not a good fit to isotopic abundance observations. Thus, this material must remain part of the ensuing supernova remnant if current theory is correct. As we have noted earlier, explosive silicon burning produces significant amounts of the $N=Z$ nucleus ${ }^{56} \mathrm{Ni}$, which decays to ${ }^{56} \mathrm{Co}$ and then to ${ }^{56} \mathrm{Fe}$. These and other radioactivities power at least some supernova light curves (e.g., SN 1987a). (See, for example, Woosley et al., 1981.)

During core silicon burning, helium, carbon, and oxygen shell burning can occur in massive stars, and the 
elements found by optical or x-ray observations to be enhanced in many supernova nebular remnants fit rather well to oxygen-shell oxygen burning, enhanced somewhat by shock wave induced explosive burning.

As the number of nuclear reactions involved in silicon burning is so large, and as many would involve very short-lived radioactive targets, the response of the experimental nuclear astrophysics community is to measure as many of the reactions as resources permit, chosen both for their own intrinsic importance, and especially as guides for theoretical efforts to devise models that can predict nuclear reaction cross sections with acceptable precision. Such models rely on a high density of states in the compound nucleus, and try to determine the way the particle decays of these states distribute themselves among the energetically allowed final states (channels). These statistical models, usually referred to as Hauser-Feshbach models, work best for $A \geq 30$ nuclei interacting with protons or alpha particles, but work quite well also for a few lighter nuclei with unusually high compound nucleus excitation energy, i.e., where the compound nuclear level density is high.

Briefly, the aim of these models is to average over compound nucleus levels. The cross section for the reaction $I^{\mu}+j \rightarrow L^{\nu}+k$, where $j$ and $k$ are particles with no excited states, can be written as (Hauser and Feshbach, 1952; Vogt, 1968; Woosley, 1986)

$$
\begin{aligned}
\bar{\sigma}_{j k}^{\mu \nu}\left(E_{j}^{\mu}\right)= & \frac{\pi \chi_{I j}^{2}}{\left(2 J_{I}^{\mu}+1\right)\left(2 J_{j}+1\right)} \\
& \times \sum_{J_{c} P}\left(2 J_{c}+1\right) \frac{T_{j}^{\mu}\left(J_{c} P\right) T_{k}^{\nu}\left(J_{c} P\right)}{T_{\mathrm{tot}}\left(J_{c} P\right)} .
\end{aligned}
$$

$T_{j}^{\mu}\left(J_{c} P\right)$ is the transmission coefficient for the incident channel to form the compound nuclear state with angular momentum $J_{c}$ and parity $P . T_{\text {tot }}\left(J_{c} P\right)$ is the sum of all transmission coefficients, including that of the entrance channel. The summation indicated is over all $J_{c}$ and $P$ in the compound nucleus. The transmission coefficients are calculated from a Schrödinger equation with proton, neutron, or $\alpha$ particle potentials provided as input. The calculation also requires a level density formula in order to carry out the indicated summation (or integration). If temperatures are high enough that many nuclei are in exicted states, partition functions are also needed. There are many other fine points that we cannot discuss here for reasons of length. A few of the various models for reaction rates are those of Truran et al. (1966); Arnett et al. (1971); Holmes et al. (1976), Woosley and Howard (1978); Mann (1976); Thielemann et al. (1986); Rauscher et al. (1997). The results of the most recent models seem to be roughly comparable.

As far as experiment is concerned, we can also only cite a sampling of the many papers from a variety of sources, including Caltech (Zyskind et al., 1977), the University of Colorado (Roughton et al., 1979, Roughton et al., 1983), and the University of Melbourne (Sargood, 1982).

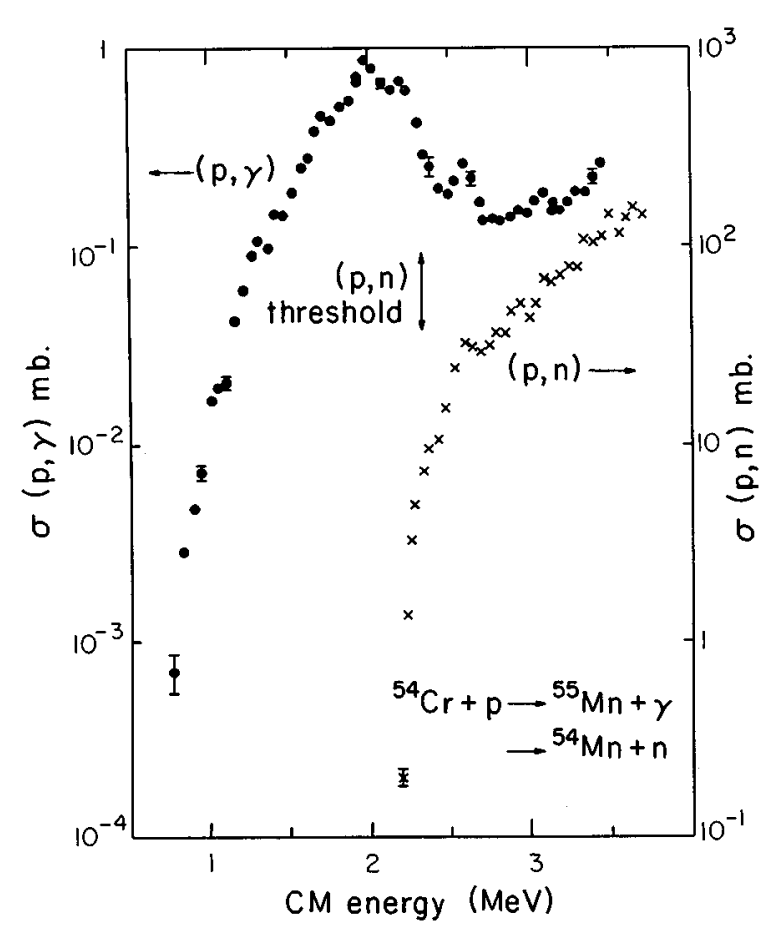

FIG. 13. Excitation functions for the reactions ${ }^{54} \mathrm{Cr}(p, \gamma){ }^{55} \mathrm{Mn}$ (black dots), and ${ }^{54} \mathrm{Cr}(p, n){ }^{54} \mathrm{Mn}$ ( $\times$ 's). The rapid decrease in the $\gamma$ ray yield beginning near $2.3 \mathrm{MeV}$ is caused by the rapid opening of the neutron channel for breakup of the compound nucleus.

Figure 13 shows an example of a phenomenon that Fowler and Caltech colleagues have named competition cusps. The gamma-ray yield from the reaction ${ }^{54} \mathrm{Cr}+p \rightarrow{ }^{55} \mathrm{Mn}+\gamma$ shows an abrupt decrease (by about a factor of 10 from the level it would otherwise have reached) beginning at the threshold for the reaction ${ }^{54} \mathrm{Cr}+p \rightarrow{ }^{54} \mathrm{Mn}+n$ (the structure is not a true mathematical cusp, and the name is purely descriptive). When such structures were discovered, it was immediately clear that they would provide sensitive tests of the models of the decay of the compound nucleus, requiring all transmission coefficients to be essentially correct.

Some of the most recent work of the Melbourne group is summarized in a paper by Hansper et al. (1997) who have made a new search to improve the nuclear potentials used in the Hauser*4 model they have chosen for comparison (Mann, 1976). In the cases studied, they have reduced the deviations from unity of the ratio $\sigma_{\text {th }} / \sigma_{\exp }$ to less than a factor of 2 . For some of the reactions at least, the deviations appear to be systematic in compound nucleus excitation energy; further improvements in the models may thus be possible.

To some extent, the tendency of silicon burning towards quasiequilibrium means that the needed data often reduce to nuclear masses, angular momenta, and other structural properties. However, there is little doubt that the need to include nuclear reaction rates for nuclei far from the locus of the stable nuclei in the $Z-N$ plane will lead to continuing demands for both experimental and theoretical improvements in silicon-burning reaction rates, and for other reactions among still 
heavier nuclei as well. We can anticipate that the new radioactive beam facilities now on line, under construction, or planned will lead to further clarification of the reaction rates for radioactive or isomeric nuclei.

As noted in Sec. VI of this review, the carbon to oxygen ratio produced in helium burning significantly affects the subsequent nucleosynthesis, and possibly the ratio of black hole to neutron star supernova remnants for massive stars (Weaver and Woosley, 1993). If the carbon to oxygen ratio is less than a few percent, core carbon and neon burning will essentially be skipped, and core oxygen burning involving more core material ensues, leading to a larger iron core, and lowering the initial stellar mass required to form black-hole remnants.

\section{$X . s$ PROCESS: LABORATORY STUDIES AND STELLAR MODELS}

\section{A. The $s$ process since $\mathrm{B}^{2} \mathrm{FH}$}

Essentially all relevant features of the slow neutron capture process were already considered by $\mathrm{B}^{2} \mathrm{FH}$. They included the stellar $(n, \gamma)$ cross sections as the important nuclear physics input, the canonical $s$-process model, the neutron sources in the helium-burning zones and the observations of $s$-process enhancements in stellar atmospheres.

This section concentrates on the nuclear physics input and its consequences for the closely related canonical or classical model, which is based on the empirical assumption that a certain fraction of the original iron was exposed to an appropriate neutron irradiation at constant temperature. It is impressive to remember the surprisingly detailed conclusions derived by $\mathrm{B}^{2} \mathrm{FH}$ despite the very uncertain input data available 40 years ago. The product of the stellar cross section and the resulting abundance, $\langle\sigma\rangle_{(A)} N_{s(A)}$, was clearly identified as the characteristic quantity of the $s$ process. Two different $s$-process components were inferred with the "local approximation" being valid between magic neutron numbers. Finally, the information that can be deduced from branchings in the synthesis path was outlined. More quantitative conclusions could not be obtained at that time, mostly due to severe uncertainties in the stellar $(n, \gamma)$ cross sections.

This major uncertainty in $s$-process studies has been greatly reduced. Neutron physics in the astrophysically relevant energy regime from about $0.1 \mathrm{keV}$ to a few hundred $\mathrm{keV}$ has reached a relatively mature stage. The advent of suitable accelerators and detector technologies led to the present situation where the stellar $(n, \gamma)$ rates for $s$-process applications start to be sufficiently reliable for interpreting the observed abundance patterns as a critical test for models of stellar helium burning. If the current efforts in this field are pursued for another decade, then the quality of the data will reach a satisfactory level.

One problem, however, that was not anticipated by $\mathrm{B}^{2} \mathrm{FH}$ was the enhancement of $\beta$ decay rates at stellar temperatures. In typical $s$-process environments, such enhancements result from transitions between individual, low-lying nuclear states that are populated by the hot photon bath. So far, theoretical estimates for these environments can be tested by only a limited number of experiments. In view of its importance for the interpretation of $s$-process branchings, this topic should be reexamined.

At present, the stellar rates for $(n, \gamma)$ reactions and $\beta$ decays allow one to draw an increasingly quantitative picture of the $s$ process. This holds even for the canonical model despite the simple and schematic assumptions on which it is based. Within the uncertainties of the input data this model is capable of describing the $s$-process abundance distribution as well as the various branchings. Only recently have accurate cross-section measurements led to significant inconsistencies with certain abundance features. At the same time, refined astronomical observations and the discovery of a variety of isotopic anomalies in meteoritic material confirmed the limited applicability of this approach. Further progress, therefore, has to be based on more realistic $s$-process modeling with advanced stellar codes.

\section{B. Laboratory experiments}

\section{Neutron capture cross sections}

Though produced with high initial energies predominantly in the exothermic $(\alpha, n)$ reactions on ${ }^{13} \mathrm{C}$ and ${ }^{22} \mathrm{Ne}$, stellar neutrons are quickly thermalized under $s$-process conditions. According to the range of thermal energies from $\approx 8 \mathrm{keV}$ in low mass stars to $80 \mathrm{keV}$ during carbon burning in massive stars, laboratory studies of stellar $(n, \gamma)$ cross sections cover the neutron energy range up to a few hundred $\mathrm{keV}$.

Neutrons in the keV range are produced in the laboratory in several ways. Nuclear reactions, such as ${ }^{7} \mathrm{Li}(p, n){ }^{7} \mathrm{Be}$ in connection with low-energy particle accelerators offer the possibility of tailoring the neutron spectrum exactly to the energy range of interest; this has the advantage of low backgrounds. Therefore, comparably short neutron flight paths can be used in these cases to compensate for the limitations in neutron flux. Measurements with this approach in Oak Ridge by Macklin and Gibbons (1967) provided the first comprehensive set of stellar cross sections for $s$-process studies. Presently, this neutron source is extensively used, for example, at the pulsed electrostatic accelerators in Karlsruhe, Germany (e.g., Käppeler, Beer, and Wisshak, 1989) and Tokyo, Japan (e.g., Nagai et al., 1991).

Much higher intensities can be achieved at linear accelerators via $(\gamma, n)$ reactions by bombarding heavy metal targets with electron beams of typically $50 \mathrm{MeV}$. When these very energetic neutrons are slowed down by a moderator the resulting spectrum contains all energies from thermal to near the initial electron energy. With respect to $s$-process data, the ORELA accelerator in Oak Ridge was the most productive facility where numerous $(n, \gamma)$ measurements were carried out by Macklin and collaborators (see, for example, the compilation 
of Bao and Käppeler, 1987). Another intense neutron source is provided by spallation reactions induced by high energy particles. The very high neutron fluxes achieved at the LANSCE facility in Los Alamos allow even time-of-flight (TOF) studies on radioactive samples (e.g., Koehler et al., 1994).

The experimental methods for measuring $(n, \gamma)$ cross sections fall into two groups, TOF techniques and activations. TOF techniques are applicable to all stable nuclei. Such measurements require a pulsed neutron source for determining the neutron energy via the flight time between target and detector. Capture events in the samples are identified by the prompt $\gamma$-ray cascade in the product nucleus. The differential data, $\sigma\left(E_{n}\right)$, obtained in TOF experiments are then folded with the Maxwellian neutron spectra for various stellar temperatures to determine the effective stellar cross sections. In general, the Maxwellian averaging for the "standard" thermal energy of $k T=30 \mathrm{keV}$ requires data in the energy range from a few $\mathrm{keV}$ up to $\sim 200 \mathrm{keV}$. Recent stellar models suggest, however, that the $s$ process may partly operate at much lower temperatures corresponding to $k T=8 \mathrm{keV}$, which means that stellar cross sections are also affected by contributions from lower energies.

A completely different approach for the determination of stellar $(n, \gamma)$ rates is by activation in a quasistellar neutron spectrum. Compared to the techniques based on the prompt capture $\gamma$ rays, this method offers the advantages of superior sensitivity (which means that much smaller samples can be measured reliably-an important aspect for the investigation of radioactive isotopes on the $s$-process path), and of selectivity (which means that samples of natural composition can be studied instead of expensive enriched samples required by the TOF techniques). However, it is restricted to those cases where neutron capture produces an unstable nucleus.

\section{Stellar $\beta$ decay}

The idea that isotopes, that are exposed to high temperatures and densities in a stellar plasma may experience a dramatic enhancement of their decay rate originated in the forties (Daudel et al., 1947), but more detailed investigations started much later (Bahcall, 1961; Bahcall, 1964a, 1964b). The enhancement results from different effects related to the thermal population of excited nuclear states and to the high degree of ionization.

In 1987, a comprehensive tabulation of stellar decay rates for $s$-process studies by Takahashi and Yokoi (1987) provided the necessary basis for a systematic discussion of $s$-process branchings. Such branchings result from the competition between neutron capture and $\beta$ decay whenever the $s$-process reaction path encounters an unstable isotope with an effective half-life comparable to the respective neutron capture time. In addition to previously studied branchings, the work of Takahashi and Yokoi (1987) revealed a number of unexpected

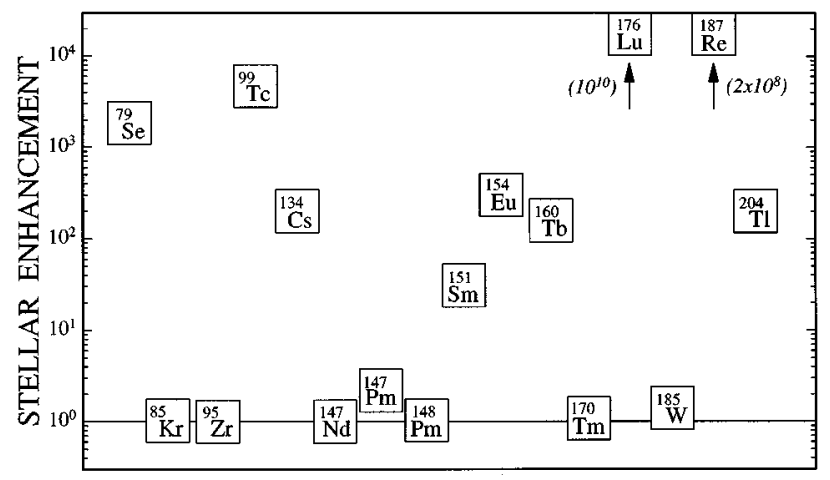

FIG. 14. The stellar enhancement factors (stellar decay rate/ terrestrial rate) for some important branch point nuclei on the $s$-process path at $k T=30 \mathrm{keV}$.

cases where terrestrially stable isotopes such as ${ }^{163} \mathrm{Dy}$ and ${ }^{179} \mathrm{Hf}$ become unstable under $s$-process conditions.

Figure 14 shows the stellar enhancement factors, i.e., the ratios of the stellar decay rates and the terrestrial rates, for the most important branch point nuclei. Obviously, there are two groups, one showing almost no enhancement at $s$-process temperatures and a second with a significant acceleration of the decay. Accordingly, the abundance patterns of the first type of branchings is suited for the determination of the $s$-process neutron density, while the second group can be interpreted in terms of the $s$-process temperature. The enhancement factors differing by large factors provides an additional test for the consistency of the branching analyses and, hence, for the investigated model.

Thermal enhancement effects are difficult to study in the laboratory since in practically all cases excited nuclear states decay much faster via $\gamma$ emission than by $\beta$ decay. The only experimentally investigated example for a $\beta$ decay from an excited state is ${ }^{79} \mathrm{Se}$. In this isotope, the enhancement is determined by the $\beta$ decay branch from the $96 \mathrm{keV}$ isomer $\left(t_{1 / 2}=4 \mathrm{~min}\right)$ which could be detected in a reactor experiment (Klay and Käppeler, 1988).

An indirect determination of a stellar lifetime was carried out for ${ }^{176} \mathrm{Lu}$ where thermal excitation of the shortlived, $\beta$-unstable isomer was quantified by a detailed study of the level scheme by means of several reactions. In this case, it was shown that the half-life of the potential $s$-process chronometer ${ }^{176} \mathrm{Lu}\left(t_{1 / 2}=36 \mathrm{Gyr}\right)$ reduces to a few years at $s$-process temperatures. Accordingly, the observed ${ }^{176} \mathrm{Lu}$ abundance can be interpreted as a thermometer for the $s$-process site (Klay et al., 1991).

The capability to accelerate and to store relativistic heavy ions at GSI Darmstadt, Germany, gave us the opportunity to demonstrate the bound state $\beta$ decay. If these terrestrially stable isotopes are completely stripped of their electrons, the electron binding energy adds to their marginally negative $Q_{\beta}$ values, thus enabling the emission of electrons into the unoccupied orbits. In the GSI measurements, stripped ions were accumulated in the storage ring and their decay followed over a period of several hours. In this way, the half-lives 
for bound state decay could be measured for ${ }^{163} \mathrm{Dy}$ (Jung et al., 1992) and ${ }^{187} \operatorname{Re}$ (Bosch et al., 1996), and were found in reasonably good agreement with the calculated values of Takahashi and Yokoi (1987). In all other cases, the stellar enhancement was calculated by using $\beta$-decay matrix elements for the excited states that were derived via the systematics of known analogous transitions. The detailed discussion of these problems and uncertainties by Takahashi and Yokoi (1987) should be considered as the basis for further theoretical and experimental improvements.

\section{The canonical s process}

\section{The $\sigma \mathrm{N}$ curve}

The first attempt at a quantitative description of the $s$ process dates back to $\mathrm{B}^{2} \mathrm{FH}$, and was later improved mainly by Seeger, Fowler, and Clayton (1965). In this approach it is empirically assumed that a certain fraction $G$ of the original stellar ${ }^{56} \mathrm{Fe}$ was irradiated by an exponential distribution of neutron exposures. In this case, an analytical solution can be obtained if a possible time dependence of the neutron capture rates, $\lambda_{n}=n_{n}\langle\sigma\rangle v_{T}$, is neglected. In other words, it is assumed that temperature and neutron density, $n_{n}$, are constant. Then the product of stellar cross section and resulting $s$ abundance, which is the characteristic $s$-process quantity, can be given by

$$
\langle\sigma\rangle_{(A)} N_{s(A)}=\frac{G \cdot N_{56}^{\odot}}{\tau_{0}} \prod_{i=56}^{A}\left(1+\frac{1}{\tau_{0}\langle\sigma\rangle_{i}}\right)^{-1},
$$

where $G$ is the fraction of ${ }^{56} \mathrm{Fe}$ abundance required as the $s$-process seed, $N_{56}^{\odot}$ is the solar ${ }^{56} \mathrm{Fe}$ abundance, and $\tau_{0}$ is the mean neutron exposure in units of $\mathrm{mb}^{-1}$.

Apart from the two parameters $G$ and $\tau_{0}$ (which are adjusted by fitting the abundances of the $s$-only nuclei), the only remaining input for this expression is the stellar $(n, \gamma)$ cross sections $\langle\sigma\rangle$. Further details may be found in Käppeler et al. (1989).

Given the very schematic nature of this classical approach, it was surprising to see that it provides an excellent description of the $s$-process abundances. Figure 15 shows the calculated $\langle\sigma\rangle N_{s}$ values compared to the corresponding empirical products of the $s$-only nuclei (symbols) in the mass region between $A=56$ and 209. The error bars of the empirical points reflect the uncertainties of the abundances and of the respective cross sections. One finds that equilibrium in the neutron capture flow was obtained between magic neutron numbers, where the $\langle\sigma\rangle N_{s}$ curve is almost constant. The small cross sections of the neutron magic nuclei around $A \sim 88,140$, and 208 act as bottlenecks for the capture flow, resulting in the distinct steps of the $\sigma N$ curve.

The global parameters $G$ and $\tau_{0}$, which determine the overall shape of the $\langle\sigma\rangle N_{s}$ curve, represent a first constraint for the stellar $s$-process site with respect to the required seed abundance and total neutron exposure. It is found that $0.04 \%$ of the observed ${ }^{56} \mathrm{Fe}$ abundance are a sufficient seed, and that on average about 15 neutrons

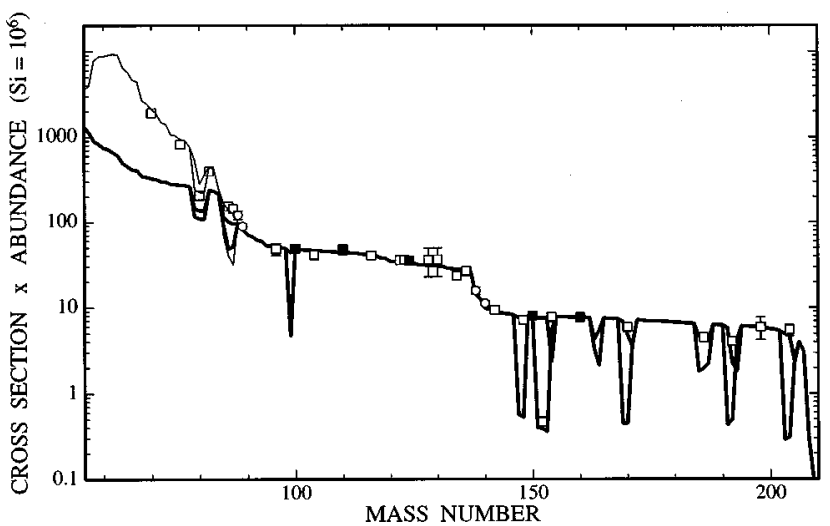

FIG. 15. The characteristic product of cross sections times $s$-process abundance plotted as a function of mass number. The solid line was obtained via the classical model, and the symbols denote the empirical products for the $s$-only nuclei. Some important branchings of the neutron capture chain are indicated as well.

are captured by each seed nucleus (Käppeler et al., 1990). These numbers refer to the main $s$-process component given by the thick line in Fig. 15. For $A<90$, this line falls below the empirical points, and these discrepancies require an additional, the so-called weak, component. Given the large number of nuclei between Fe and Bi that are described with very few parameters, the excellent agreement between the empirical points and the data obtained with the classical model is a unique feature of the $s$ process. In fact, those $s$-only nuclei that are not affected by branchings are reproduced by the model with a mean square deviation of only 3\% (Käppeler et al., 1990).

In terms of stellar sites, the main component can be attributed to helium shell burning in low mass stars (Iben and Renzini, 1983; Hollowell and Iben, 1989). In this stage of evolution, enery is produced in a narrow double shell is produced on top of an inert core consisting of ${ }^{12} \mathrm{C}$ and ${ }^{16} \mathrm{O}$. Hydrogen burning at the bottom of the deeply convective envelope produces helium that accumulates in a thin layer around the core. As soon as this layer exceeds a critical mass, helium burning is ignited leading to a highly unstable situation because of the large associated energy production. As a result, strong convective motions cause practically instantaneous mixing in the helium-burning layer, and eventually freshly synthesized matter is dredged up to the surface (third dredge-up, see Sec. III). While helium burning lasts for only about $200 \mathrm{yr}$, it takes about $5 \times 10^{4}$ yr for hydrogen burning to replenish the consumed helium. These helium-burning episodes can repeat up to about 20 times.

Neutron production and concordant $s$ processing occur in two steps: by the ${ }^{13} \mathrm{C}(\alpha, n){ }^{16} \mathrm{O}$ reaction during the hydrogen-burning stage at relatively low temperatures of $\mathrm{T}_{8} \sim 1$ and during the subsequent helium burning by the ${ }^{22} \mathrm{Ne}(\alpha, n)^{25} \mathrm{Mg}$ reaction $\left(\mathrm{T}_{8} \sim 3\right)$. The resulting $s$-process abundances have been investigated by numerous papers from the Torino group (e.g., Gallino et al., 


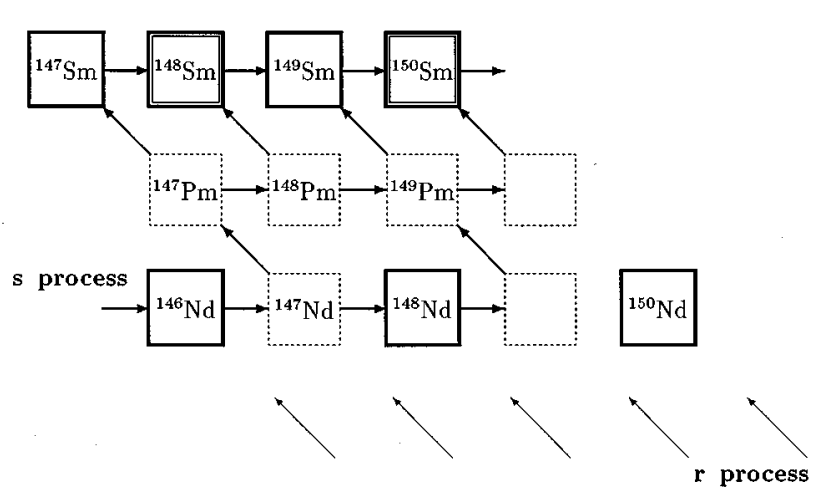

FIG. 16. The $s$-process reaction path in the $\mathrm{Nd} / \mathrm{Pm} / \mathrm{Sm}$ region with the branchings at $A=147,148$, and 149 . Note that ${ }^{148} \mathrm{Sm}$ and ${ }^{150} \mathrm{Sm}$ are shielded against the $r$ process. These two isotopes define the strength of the branching.

1988; Gallino, 1989; Straniero et al., 1995; Gallino et al., 1996).

As a consequence of the comparably short time scale and the strong impact of convection, stellar models for the red giant phase are very different from the assumption of a steady situation made in the classical approach. In order to account for the more complex stellar scenario, the canonical model was recently complemented by a network code (Jaag, 1990) and by a parametrized model (Beer et al., 1997) for describing the combined action of the pulsed ${ }^{13} \mathrm{C}$ and ${ }^{22} \mathrm{Ne}$ sources.

The weak component has been ascribed to core helium burning in massive stars (Peters, 1968; Couch, Schmeidekamp, and Arnett, 1974; Lamb et al., 1977; Langer et al., 1989; Prantzos et al., 1990; El Eid and Baraffe, 1990; Raiteri et al., 1993) when the temperatures become sufficiently high for neutron production via the ${ }^{22} \mathrm{Ne}(\alpha, n){ }^{25} \mathrm{Mg}$ reaction. The resulting neutron density is comparatively low and lasts for about $3 \times 10^{4} \mathrm{yr}$, resulting in the modest neutron exposure of the weak $s$-process component.

The models for the scenarios suggested for the main and weak components can best be tested by detailed analyses of the $s$-process branchings that occur in the respective mass regions.

\section{Branchings}

A branching in the reaction flow occurs when an unstable nucleus that exhibits comparable neutron capture and $\beta$ decay rates is encountered. The resulting abundance pattern can be used to determine information on the physical conditions during the $s$ process. The example of Fig. 16 shows the $s$-process flow in the mass region between neodymium and samarium, with the possible branchings at ${ }^{147} \mathrm{Nd}$ and ${ }^{147-149} \mathrm{Pm}$. Note that ${ }^{148} \mathrm{Sm}$ and ${ }^{150} \mathrm{Sm}$ are shielded against the $r$ process by their isobars in neodymium. As the result of a significant branching at $A=147-149$, the $\langle\sigma\rangle N_{s}$ value of ${ }^{150} \mathrm{Sm}$ will, therefore, always be larger than that of ${ }^{148} \mathrm{Sm}$.

The strength of a branching can be expressed in terms of the rates for $\beta$ decay and neutron capture of the in- volved branch point nuclei as well as by the $\langle\sigma\rangle N_{s}$ values of the involved $s$-only isotopes,

$$
f_{\beta}=\frac{\lambda_{\beta}}{\lambda_{\beta}+\lambda_{n}} \approx \frac{\left(\langle\sigma\rangle N_{s}\right)_{148 \mathrm{Sm}}}{\left(\langle\sigma\rangle N_{s}\right)_{150 \mathrm{Sm}}}
$$

(Clayton, 1968). Inserting the equations for the decay rates, this expression can be solved for the neutron density $n_{n}$. If, for simplicity, only the branching at ${ }^{148} \mathrm{Pm}$ is considered, one obtains

$$
n_{n}=\frac{1-f_{\beta}}{f_{\beta}} \frac{1}{v_{T}\langle\sigma\rangle_{148 \mathrm{Pm}}} \frac{\ln 2}{t^{*}{ }_{1 / 2\left({ }^{148} \mathrm{Pm}\right)}} .
$$

This equation demonstrates the input data that are important for reliable branching analyses.

The first term depends on the cross sections for the $s$-only nuclei, which define the branching factor $f_{\beta}$. Since the neutron density is required to be $\sim 10 \%$, the branching factor, and hence the cross sections for the $s$-only nuclei need to be known to about $1 \%$ in many cases. While conventional techniques are limited to uncertainties $>4 \%$, new developments such as a $4 \pi \mathrm{BaF}_{2}$ detector (Wisshak et al., 1990) or-in favorable casesthe activation technique allow measurements of the required accuracy.

The second term contains the stellar cross sections of the radioactive branch point isotopes. Since there are practically no measurements for the unstable branch point nuclei, only calculated cross sections are available at present. Even the most careful statistical model calculations are limited to uncertainties of $20 \%$ to $30 \%$, not sufficient for deducing the entire information contained in the abundance patterns. For some of these short-lived nuclei, experimental cross section studies aiming at a $5 \%$ to $10 \%$ uncertainty were recently suggested (Jaag and Käppeler, 1995; Käppeler, 1992).

The last term denotes the stellar decay rate of the branch point isotope. While there is no difference between the stellar and the terrestrial rate for some of the branch points, a variety of examples sometimes exhibit drastic changes under the high temperatures and densities of the stellar plasma (Fig. 14).

Since the $\beta$ decay rates of the branch points at $A=147-149$ in Fig. 16 are not significantly affected by temperature, these branchings can be used for a determination of the $s$-process neutron density. Compared to the previous result of Winters et al. (1986) $\left(f_{\beta}=0.92 \pm 0.04\right)$, the measurement with a $4 \pi \mathrm{BaF}_{2}$ detector yields $f_{\beta}=0.870 \pm 0.009$, with a four times smaller uncertainty (Wisshak et al., 1993). This branching factor implies a neutron density of $n_{n}=(4.1 \pm 0.6) \times 10^{8} \mathrm{~cm}^{-3} \quad$ (Toukan et al., 1995), in agreement with the previously estimated $(3.4 \pm 1.1) \times 10^{8}$ $\mathrm{cm}^{-3}$ (Käppeler et al., 1990). An example of a branching that is readily observable in stellar atmospheres occurs at ${ }^{85} \mathrm{Kr}$ as described by V. V. Smith in Sec. XI.

Along the $s$-process path there are about 15 to 20 significant branchings which can be studied with respect to the physical conditions at the stellar site. In a first 
TABLE V. Results from various branching analyses of relevance for the main $s$-process component.

\begin{tabular}{lll}
\hline \hline Branch point isotope & Deduced $s$-process parameter & \multicolumn{1}{c}{ Reference } \\
\hline${ }^{147} \mathrm{Nd} /{ }^{147} \mathrm{Pm} /{ }^{148} \mathrm{Pm}$ & $n_{n}=(4.1 \pm 0.6) \times 10^{8} \mathrm{~cm}^{-3}$ & Toukan et al. $(1995)$ \\
${ }^{151} \mathrm{Sm} /{ }^{154} \mathrm{Eu}$ & $T_{8}=3.5 \pm 0.4$ & Wisshak et al. $(1995)$ \\
${ }^{163} \mathrm{Dy} /{ }^{163} \mathrm{Ho}$ & $\rho_{s}=(6.5 \pm 3.5) \times 10^{3} \mathrm{gcm}^{-3}$ & Jaag and Käppeler $(1996)$ \\
${ }^{176} \mathrm{Lu}$ & $T_{8}=3.1 \pm 0.6$ & Klay et al. $(1991)$ \\
& & \\
${ }^{121} \mathrm{Sn} /{ }^{122} \mathrm{Sb}$ & $T_{8}>2.4$ & Käppeler et al. $(1993)$ \\
${ }^{134} \mathrm{Cs}$ & $T_{8}=1.9 \pm 0.3$ & Voss et al. $(1994)$ \\
& $T_{8}=1.7 \pm 0.5$ & Koehler et al. $(1996)$ \\
${ }^{185} \mathrm{~W} /{ }^{186} \mathrm{Re}$ & $n_{n}=\left(3.5_{-1.1}^{+1.7}\right) \times 10^{8} \mathrm{~cm}^{-3}$ & Käppeler et al. $(1991)$ \\
\hline \hline
\end{tabular}

step, the neutron density must be obtained from those branchings that are not affected by temperature. With this information, the effective branching factor of the remaining cases can be derived and the mean stellar decay rates be determined. Eventually, the dependence of these rates on temperature and/or electron density yields the $s$-process temperature and mass density (Käppeler et al., 1990). The present status of branching analyses with the classical approach is summarized in Table V. Prominent examples in this list are from the lanthanide region, which is particularly important in this context because the chemical similarity of these elements implies that their relative abundances are well defined. Therefore, the total reaction flow can be defined by ${ }^{150} \mathrm{Sm}$, analogously to the situation of Fig. 16. Such branchings occur at $A=141,151,154,163$, and 169 and are defined by the $s$ isotopes ${ }^{142} \mathrm{Nd},{ }^{152} \mathrm{Gd},{ }^{154} \mathrm{Gd},{ }^{164} \mathrm{Er}$, and ${ }^{170} \mathrm{Tm}$, respectively.

As far as the neutron density is concerned the best value in Table $\mathrm{V}, n_{n}=(4.1 \pm 0.6) \times 10^{8} \mathrm{~cm}^{-3}$, is in good agreement with the estimate obtained from the ${ }^{185} \mathrm{~W} /$ ${ }^{186} \mathrm{Re}$ branching. The various estimates of the $s$-process temperature are also compatible with each other, except for the result from the ${ }^{134} \mathrm{Cs}$ branching. This discrepancy, however, may originate from an uncertainty in the temperature-dependent decay rate of ${ }^{134} \mathrm{Cs}$ which had been noted explicitly by Takahashi and Yokoi (1987). Hence, this problem should be investigated further before the ${ }^{134} \mathrm{Cs}$ branching can be discussed in any quantitative way.

A first attempt to determine the mass density in the $s$ process was carried out by Beer et al. (1985) by analyzing a small branching to ${ }^{164}$ Er. Their value, $\rho_{s}=(8 \pm 5) \times 10^{3} \mathrm{~g} \mathrm{~cm}^{-3}$, is in reasonable agreement with the recent analysis cited in Table V (Jaag and Käppeler, 1996).

So far, the results of the different branching analyses in Table $\mathrm{V}$ seem to be compatible with each other, pointing to the physical conditions typical of the ${ }^{22} \mathrm{Ne}(\alpha, n){ }^{25} \mathrm{Mg}$ neutron source. In a way, this apparent success also marks the limitation of the classical model: it can only interpret the final abundance pattern. Being thus restricted to the last modification of the $s$ abundances, the dominance of the ${ }^{13} \mathrm{C}(\alpha, n){ }^{16} \mathrm{O}$ source is missed, in clear contradiction to observation (see Sec.
$\mathrm{XI})$. Though there are a few features from the ${ }^{13} \mathrm{C}$ era surviving the ${ }^{22} \mathrm{Ne}(\alpha, n)$ episode-especially near magic neutron numbers where cross sections are small-these hints are too weak for reconstructing the true stellar $s$ process. This limitation is common to all attempts of reconstructing the $s$ process from the observed abundance pattern alone.

Accordingly, progress in understanding the physics of the $s$ process can only be achieved by means of stellar models. But since these models are still plagued by a number of open questions, further laboratory studies have to concentrate on the aspect of constraining critical parameters in these calculations. In particular, theoretical concepts of convection and mixing may be tested via their effect on $s$-process branchings. In any case, the measurements have to account for the lower temperatures during the ${ }^{13} \mathrm{C}(\alpha, n)$ phase.

\section{OBSERVATIONS OF THE $s$ PROCESS}

\section{A. Brief history}

At the time of the writing of $\mathrm{B}^{2} \mathrm{FH}$, one of the key observations - that many of the elements heavier than He might be produced in stars-was Merrill's (1952) classic, and often cited, spectroscopic detection of neutral technetium in certain red giant stars of spectral type S. As technetium consists only of cosmically short-lived isotopes $\left(t_{1 / 2} \sim 10^{5}-10^{6} \mathrm{yrs}\right)$, the Tc I observed by Merrill had been "recently" synthesized in the interiors of the S stars and mixed to their surfaces. The Tc in the S stars, and the other neutron-rich heavy elements observed to be overabundant in many types of red giants (e.g., $\mathrm{Zr}$, $\mathrm{Ba}$, or $\mathrm{La}$ ), was created at low neutron densities and is a product of $\mathrm{B}^{2} \mathrm{FH}$ 's $s$ process.

It has been known since the work of Schwarzschild and Härm (1967) and Sanders (1967) that the preferred astrophysical site for the production of many of the $s$-process nuclei was in a region associated with the $\mathrm{H}$ and ${ }^{4} \mathrm{He}$-burning shells found in asymptotic giant branch (AGB) stars. Much of the early work which connected the AGB stellar models to the details of the $s$-process nucleosynthesis was carried out by Iben and collaborators and is summarized nicely in the review by Iben and Renzini (1983). 
This is a brief review of a few of the recent observational results of the $s$ process in AGB stars.

\section{B. Observations of nucleosynthesis and mixing in $\mathrm{CH}, \mathrm{Ba}$, $\mathrm{S}$, and $\mathrm{C}$ stars}

Of the various nuclear processes characterized by $\mathrm{B}^{2} \mathrm{FH}$, the $s$ process is perhaps one of the easiest to study using direct observations of stars. Because the $s$ process has the greatest effect on the relatively minor abundance elements heavier than Fe, such as $\mathrm{Y}, \mathrm{Zr}, \mathrm{Ba}$, or La (all of which have readily accessible spectral lines), rather small abundance changes of a factor of 2 , or more, can be quickly detected by straightforward spectroscopic techniques. Also, the $s$ process is activated during the relatively stable phase of stellar evolution along the AGB, thus there are a variety of stars that can be studied which bear the spectroscopic signs of the mixing of freshly synthesized $s$-process elements to their surfaces. The result of $s$-process nucleosynthesis in AGB stars has left its mark on a number of stellar classes which exhibit overabundances of the classic $s$-process nuclei, although of course it has only been since the linking of the $s$ process to evolution along the AGB that these rather diverse stellar types have been found to have common origins.

As quasiperiodic shell ${ }^{4} \mathrm{He}$ burning occurs during thermal pulses on the AGB, mixing episodes increase the atmospheric $\mathrm{C} / \mathrm{O}$ ratio in these red giants, giving rise to the spectral sequence of $\mathrm{M}$ to $\mathrm{MS}$ to $\mathrm{S}$ to $\mathrm{C}$. This increase in $\mathrm{C} / \mathrm{O}$ is accompanied, in general, by an increasing $s$-process overabundance, thus the MS, S, and $\mathrm{C}$ stars are heavy element rich AGB stars. Carbon stars have been known for well over a century, while the intermediate $\mathrm{C} / \mathrm{O}$ ratio $\mathrm{S}$ stars were first isolated as a specific stellar type by Merrill (1922) and their spectral cousins the MS stars were discussed by Keenan (1954). Other warmer, heavy element rich stellar classes are known; Bidelman and Keenan (1951) identified the barium $(\mathrm{Ba})$ giants as being heavy element $(\mathrm{Ba}$ and $\mathrm{Sr})$ rich, and soon after it was realized that the $\mathrm{CH}$ stars discovered and classified by Keenan (1942) were metalpoor halo relatives of $\mathrm{Ba}$ stars. In more recent times, Bond (1974) found subgiant and near main-sequence relatives of the giant $\mathrm{CH}$ and $\mathrm{Ba}$ stars and dubbed them $\mathrm{CH}$ subgiants. Since Bond's discovery, many more true main-sequence $s$-process rich stars of spectral types $\mathrm{F}$ and $G$ have been identified: Tomkin et al. (1989) or North et al. (1994). Observationally defined s-process rich stars thus span the range in luminosity from mainsequence to extremely luminous AGB giants.

Since the $s$ process was identified with AGB evolution in the 1970's, the lower luminosity s-process rich Ba and $\mathrm{CH}$ giants and subgiants defied explanation for many years. McClure et al. (1980) broke the logjam of understanding these diverse groups when they discovered that all $\mathrm{Ba}$ stars were binaries with white dwarf companions. The solution to the $s$-process nature of these stars thus centers on mass transfer; when the white dwarf now inhabiting these binary systems was a luminous AGB star and had mixed $s$-process rich material to its surface, it transferred some of this processed matter to its companion, which is now observed as an $s$-process rich star. Since the pioneering paper of McClure et al. (1980), follow-up studies (McClure, 1984; McClure and Woodsworth, 1990) have confirmed the mass-transfer scenario. In addition, it has been shown that the $40 \%$ of S stars which do not show the lines of Tc I in their spectra are the cooler relatives of the Ba stars and result from mass transfer (Iben and Renzini, 1983; Smith and Lambert, 1988; Brown et al., 1990; Jorissen et al., 1993). Most recently, a number of the active symbiotic binaries have been identified as $\mathrm{C}$ rich (Schmid and Nussbaumer, 1993; Schmid, 1994), while the yellow symbiotic stars AG Dra and BD-21 3873 have both been shown to be $s$-process rich and thus technically Ba stars as well as symbiotics (Smith et al., 1996, 1997). There is now a coherent picture of the $s$-process rich classes of stars: the $\mathrm{N}$-type carbon stars, along with the MS and S stars with Tc, are bonafide AGB stars that have recently dredged fresh $s$-process material to their surfaces. The other variety of $s$-process stars (the $\mathrm{Ba}$ and $\mathrm{CH}$ giants, subgiants, and main-sequence stars, the carbon star dwarfs, and now the $\mathrm{C}$ and $s$-process rich symbiotic stars) result from mass-transfer in certain binary star systems.

With so many varieties of stars exhibiting clear signatures of enhancements of the $s$ process in their abundance patterns, it is not surprising that a great deal of effort has been expended by observational spectroscopists in analyzing the details of $s$-process nucleosynthesis in the heavy-element rich stars. A number of detailed abundance analyses have been conducted on the various heavy-element rich stars over the last 15 years and can be summarized as follows: for the barium giants we have Tomkin and Lambert (1983, 1986), Smith (1984), Kovacs (1985), Smith and Lambert (1984), Malaney and Lambert (1988), and Busso et al. (1995), while the mainsequence $\mathrm{Ba}$ stars have been studied by Tomkin et al. (1989) and North et al. (1994). The CH giants have been studied by Vanture (1992a, 1992b). The MS and S stars have been studied by Smith and Lambert (1990), Plez et al. (1993), Busso et al. (1995), and Lambert et al. (1995), while the most detailed analysis of the $s$ process in the carbon stars is still Utsumi (1985). Recent abundance studies have uncovered $s$-process rich post-AGB stars in the globular cluster $\omega$ Cen (Gonzalez and Wallerstein, 1994), as well as $s$-process enhancements in the yellow symbiotics (Smith et al., 1996, 1997).

Taken together, these abundance studies provide the raw material with which to probe our current understanding of the details of $s$-process nucleosynthesis in AGB stars and in the next sections we summarize these results.

\section{The $s$ process as a function of metallicity}

One question that is basic to an understanding of the $s$ process in AGB stars is the nature of the neutron source. Since the realization that the slow capture of neutrons is a distinct stellar nuclear process, two candi- 
date neutron producing reactions have been suggested: ${ }^{13} \mathrm{C}(\alpha, n){ }^{16} \mathrm{O}$ and ${ }^{22} \mathrm{Ne}(\alpha, n){ }^{25} \mathrm{Mg}$ (Cameron, 1955; Greenstein, 1954). These two separate sources have very different characters; the $s$ process in AGB stars is associated with ${ }^{4} \mathrm{He}$-burning temperatures of $T \geqslant 10^{8} \mathrm{~K}$, with the ${ }^{13} \mathrm{C}$ source being active near $1 \times 10^{8} \mathrm{~K}$, while the ${ }^{22} \mathrm{Ne}$ source requires higher temperatures nearer to $(2-3) \times 10^{8} \mathrm{~K}$. The different temperatures required to drive the $s$ process via the different neutron sources will occur in different mass AGB stars, or at different points along the AGB. Historically, the ${ }^{22} \mathrm{Ne}$ source was favored as the most likely $s$-process candidate because the production of neon-22 can occur quite naturally as the result of succesive $\alpha$ captures onto ${ }^{14} \mathrm{~N}$ in the He-burning shell of an AGB star $\left.\left[{ }^{14} \mathrm{~N}(\alpha, \gamma){ }^{18} \mathrm{~F}\left(e^{+}, \nu\right){ }^{18} \mathrm{O}(\alpha, \gamma)\right)^{22} \mathrm{Ne}\right]$, with the initial ${ }^{14} \mathrm{~N}$ itself coming from CNO-cycle $\mathrm{H}$ burning (Iben, 1975a, 1975b, 1976, 1977a, 1977b). The carbon-13 neutron source required an extra mixing mechanism to move protons into the He-burning shell to produce ${ }^{13} \mathrm{C}$ via ${ }^{12} \mathrm{C}(p, \gamma){ }^{13} \mathrm{~N}\left(\beta^{+}, \nu\right){ }^{13} \mathrm{C}$, and it was not clear as to the physical nature of this extra mixing. However, the expected behavior of these neutron sources with metallicity is different and can be tested by the observations.

A convenient parameter to characterize the relative abundance distribution of the $s$ process is the neutron exposure $\tau$ defined as

$$
\tau=\int_{0}^{t^{\prime}} N_{n}(t) V(t) d t
$$

where $N_{n}$ is the neutron density and $V(t)$ the relative velocity of neutrons and nuclei, with the integral taken over the interval of the $s$-process episode. A large neutron exposure leads to a larger amount of heavier nuclei produced (e.g., $\mathrm{Ba}, \mathrm{La}$, or $\mathrm{Ce}$ ) relative to the lighter nuclei, such as $\mathrm{Y}$ or $\mathrm{Zr}$.

As pointed out by Clayton (1988), the nature of the dominant $s$-process neutron source can be tested by using the heavy- to light-element abundance distribution of the $s$ process (a measure of $\tau$ ) as a function of metallicity. For example if, during the $s$-process episode, the neutrons are in local equilibrium between their production and destruction, then the neutron density will be

$$
N_{n}=\left(\Sigma N_{i} N_{j}\langle\sigma\rangle_{i, j}\right) /\left(\Sigma N_{k}\langle\sigma\rangle_{k, n}\right),
$$

where the numerator is summed over all neutronproducing reactions between species $i, j$, with Maxwellian-averaged cross sections of $\langle\sigma\rangle_{i, j}$, and the denominator has the neutron destruction (absorbing) reactions, summed over all neutron absorbers, $k$, and again a Maxwellian-averaged neutron absorption cross section of $\langle\sigma\rangle_{k, n}$. Making the simplifying assumption that the neutron producing reactions come from $\alpha$ captures, and that the dominant neutron absorbers are the seed nuclei of $\mathrm{Fe}$, the above expression can be approximated as

$$
N_{n} \propto\left(N_{\mathrm{He}} N_{j}\right) /\left(N_{\mathrm{Fe}}\right),
$$

where $N_{j}=N\left({ }^{13} \mathrm{C}\right)$ for the carbon-13 source and $N\left({ }^{22} \mathrm{Ne}\right)$ for the neon-22 source. Since the ${ }^{22} \mathrm{Ne}$ in the
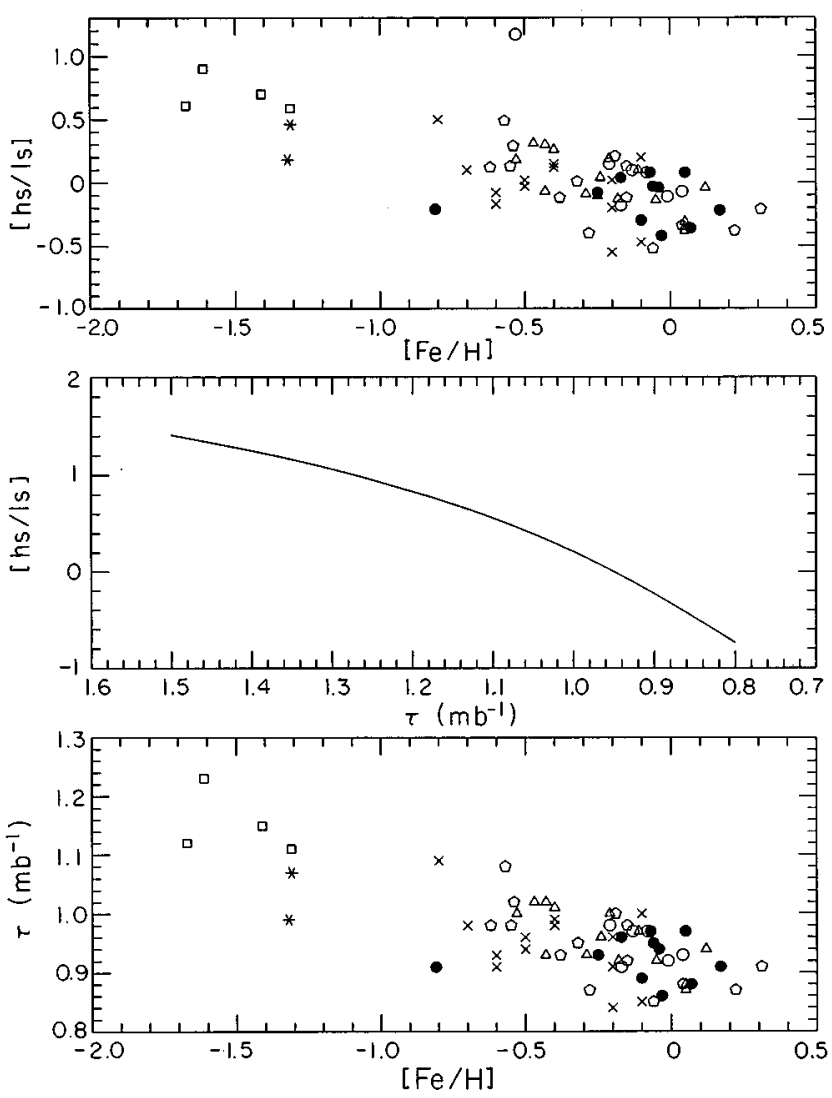

FIG. 17. Published abundance results for the heavy s-process elements $(\mathrm{Ba}, \mathrm{La}$, and $\mathrm{Ce})$ relative to the light $s$-process species ( $\mathrm{Y}$ and $\mathrm{Zr}$ ) for the MS/S stars (filled circles, with Tc, and open circles, without Tc), Ba giants (open triangles), Ba dwarfs (open pentagons), $\mathrm{CH}$ giants (open squares), $\mathrm{CH}$ subgiants (crosses), and yellow symbiotics (six-pointed stars). The middle panel shows the same quantity, [hs/ls], as in the top panel, except that the abundances come from models of $s$-process nucleosynthesis from Malaney (1987). This is plotted versus the neutron exposure, $\tau$, for a single exposure model. In the bottom panel are shown the observed values of [hs/ls] transformed to $\tau$ from Malaney's models, plotted versus [Fe/ $\mathrm{H}]$. The increase of neutron exposure with decreasing metallicity is clear.

He-burning layers comes from ${ }^{14} \mathrm{~N}$ which, itself, comes from the initial $\Sigma \mathrm{CNO}$ which is $\propto N_{\mathrm{Fe}}$, the expected neutron density (and neutron exposure) would be expected to be roughly independent of metallicity. Carbon-13, on the other hand, must result from the mixing of protons into a ${ }^{12} \mathrm{C}$-rich region, where the carbon-12 comes from ${ }^{4} \mathrm{He}$, which is almost independent of metallicity. Thus if the structures of He shells in AGB stars are not strong functions of metallicity the numerator of the above expression is constant and $N_{n}$ (and $\tau$ ) is $\propto N_{\mathrm{Fe}}^{-1}$.

The neutron exposure can be estimated using published abundance results and we show the results of such an exercise in Fig. 17. In the top panel is plotted the quantity $[\mathrm{hs} / \mathrm{ls}]$ versus $[\mathrm{Fe} / \mathrm{H}]$ from a number of studies. The value of [hs/ls] stands for heavy-s to light-s and is an average overabundance of representative heavy 
$s$-process nuclei ( $\mathrm{Ba}, \mathrm{La}$, and $\mathrm{Ce}$ ) relative to an average overabundance of the light $s$-process species $\mathrm{Y}$ and $\mathrm{Zr}$, measured in standard spectroscopic bracket notation. There is rather large scatter, although this is not surprising as we are comparing results from a large number of investigators using somewhat different techniques and different quality data. Nonetheless, there is a clear and significant trend of increasing [hs/ls] with decreasing metallicity. The quantity [hs/ls] can be mapped into a neutron exposure using the theoretical calculations from Malaney (1987), which we illustrate in the second panel: here we plot [hs/ls] defined in exactly the same way as for the stellar results, but using Malaney's model abundances, versus the single-exposure neutron exposure $\tau$. In the bottom panel, the observed $\tau$ derived from Malaney's "calibration" is plotted versus $[\mathrm{Fe} / \mathrm{H}]$. There is a significant increase of neutron exposure with decreasing metallicity as predicted from Clayton's (1988) simple argument for the ${ }^{13} \mathrm{C}(\alpha, n)$ neutron source. Based on the observations, it would appear that the current best candidate for the $s$-process neutron source in the AGB stars of low mass is carbon- 13 .

\section{Rubidium and the $s$-process neutron density}

In an $s$-process environment, for the limiting case where the neutron density goes to zero, the $s$ process follows a unique path along the valley of stability because the first $\beta$-unstable nucleus will decay rather than capture a neutron. As $N_{n}$ increases, however, certain $\beta$-unstable nuclei will either decay or capture another neutron depending on how the neutron capture rate (which increases with increasing neutron density) compares to the $\beta$ decay rate for that particular nucleus. As described in detail in Sec. X, at these critical branch points, the $s$-process path can follow somewhat different routes, and certain branch points can result in measurable abundance differences, depending on which path the $s$ process travels (which will depend on the neutron density). One such branch point that can have observable consequences occurs at ${ }^{85} \mathrm{Kr}$, which controls the $s$-process path either through ${ }^{85} \mathrm{Rb}$ or ${ }^{87} \mathrm{Rb}$. Krypton- 85 has a ground-state half-life of 10 years and $\beta$ decays to the stable nucleus ${ }^{85} \mathrm{Rb}$. If ${ }^{85} \mathrm{Kr}$ captures a neutron, however, it leads to the stable isotope ${ }^{86} \mathrm{Kr}$ which will neutron capture to ${ }^{87} \mathrm{Kr}$, which will then $\beta$ decay (in just over an hour) to stable ${ }^{87} \mathrm{Rb}$. Depending on whether ${ }^{85} \mathrm{Kr} \beta$ decays or captures a neutron, the $s$-process path will travel to either ${ }^{85} \mathrm{Rb}$ or ${ }^{87} \mathrm{Rb}$; the half-life of ${ }^{86} \mathrm{Rb}$ is 18 days (much less than ${ }^{85} \mathrm{Kr}$ ), so the low-density branch of the $s$ process bypasses ${ }^{87} \mathrm{Rb}$, while the high-density branch bypasses ${ }^{85} \mathrm{Rb}$. These two $\mathrm{Rb}$ isotopes have very different neutron capture cross sections $\left[\sigma\left({ }^{85} \mathrm{Rb}\right)=240\right.$ $\mathrm{mb}$ and $\sigma\left({ }^{87} \mathrm{Rb}\right)=21 \mathrm{mb}$ at $k T=30 \mathrm{keV}$ (Beer and Macklin, 1989), so the resultant $\mathrm{Rb}$ abundance will vary according to the ratios of their respective cross sections. Both the high- and low-density $s$-process paths go from $\mathrm{Rb}$ on to the nearby elements $\mathrm{Sr}, \mathrm{Y}$, and $\mathrm{Zr}$, so the elemental abundance ratio of $\mathrm{Rb}$ to $\mathrm{Sr}$, or $\mathrm{Y}$, or $\mathrm{Zr}$ can

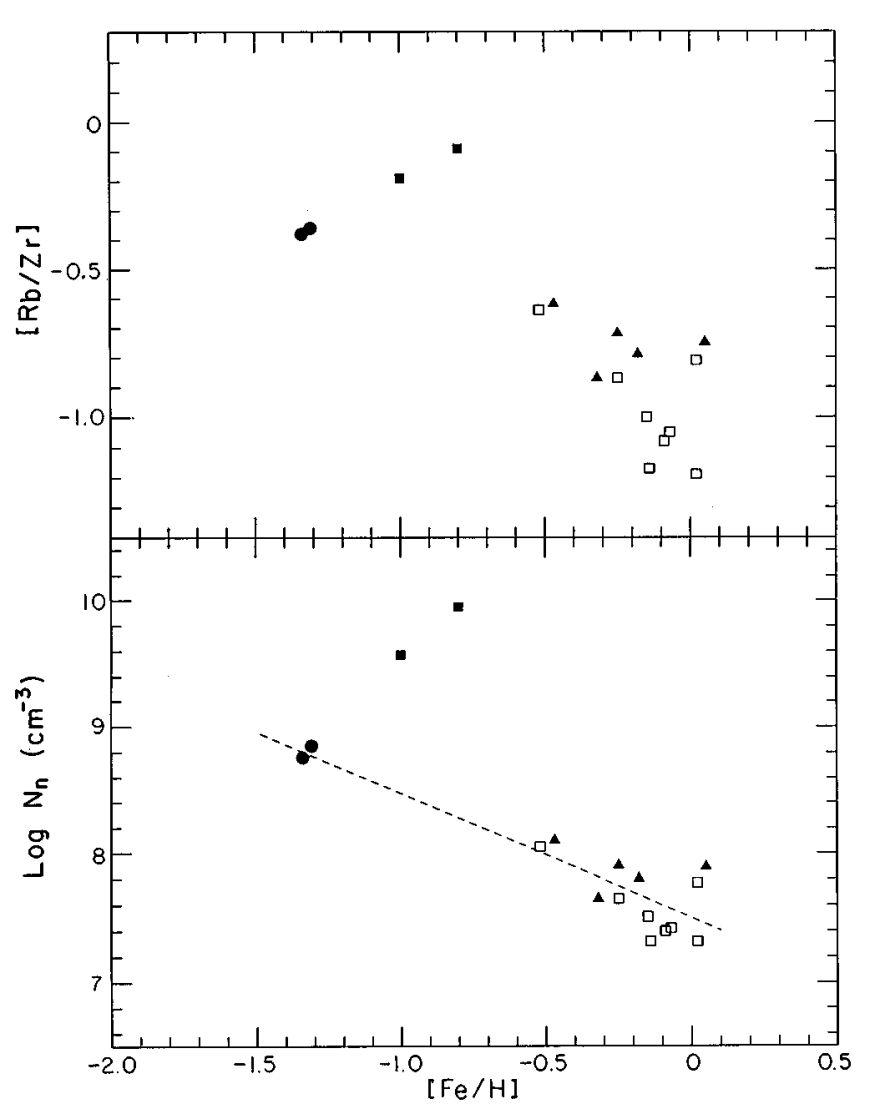

FIG. 18. The $[\mathrm{Rb} / \mathrm{Zr}] s$-process abundances versus $[\mathrm{Fe} / \mathrm{H}]$ (top panel) in the $\mathrm{Ba}$ giants (filled triangles), the MS/S stars (open squares), the yellow symbiotic stars (filled circles), and the $\omega$ Cen giants (filled squares). The increase of $[\mathrm{Rb} / \mathrm{Zr}]$ with decreasing $[\mathrm{Fe} / \mathrm{H}]$ is striking. In the bottom panel, $[\mathrm{Rb} / \mathrm{Zr}]$ has been transformed to neutron density using calculations presented in Malaney (1987) and Malaney and Lambert (1988). A linear least-squares fit to the MS/S, Ba, and yellow symbiotic stars is shown: this line has a slope of -1 . The $\omega$ Cen stars are not included in this fit because the $s$-process enrichments result from more complicated chemical evolution (see the text for a discussion).

be used as a neutron density monitor. The Rb abundance changes most rapidly between neutron densities of about $10^{7}-10^{10} \mathrm{~cm}^{-3}$.

As $\mathrm{Rb}$ is a fairly low abundance species and represented only by $\mathrm{Rb} \mathrm{I}$, whose ionization potential is 4.18 $\mathrm{eV}$, it is difficult to analyze and must be attacked using the resonance line at $7800 \AA$. In the last 15 years or so a number of results for $\mathrm{Rb}$ have become available for $\mathrm{Ba}$ stars (Tomkin and Lambert, 1983; Smith and Lambert, 1984; Malaney and Lambert, 1988), the MS and S stars (Lambert et al., 1995), $s$-process rich giants in the globular cluster $\omega$ Cen (Vanture et al., 1994), and the $s$-process rich yellow symbiotic stars (Smith et al., 1996, 1997). In addition, Wallerstein (1992) has analyzed the $\mathrm{Rb}$ I resonance line in emission in the S star, $\mathrm{R}$ Andromeda, and estimated the $\mathrm{Rb} / \mathrm{K}$ abundance ratio (using the $\mathrm{K}$ I resonance line also in emission). Wallerstein assumed that the collisional excitation and deexcitation cross sections are the same for both $\mathrm{Rb} \mathrm{I}$ and $\mathrm{K} \mathrm{I}$. Thus there is now a rather substantial set of data on the be- 
havior of $\mathrm{Rb}$, and hence the neutron density, over a range in stellar metallicity. The discussion of the previous section holds here and, for the case of the ${ }^{13} \mathrm{C}(\alpha, n)$ neutron-source, one might expect an increasing neutron density with decreasing metallicity. Results for $\mathrm{Rb}$ are summarized in Fig. 18: the top panel presents the observational quantities $[\mathrm{Rb} / \mathrm{Zr}]$ versus $[\mathrm{Fe} / \mathrm{H}]$ for the barium star references listed above (filled triangles), the MS and $S$ stars (open squares), the $\omega$ Cen giants (filled squares), and the yellow symbiotics (filled circles). The large increase of the $\mathrm{Rb} / \mathrm{Zr}$ abundance ratio with decreasing $[\mathrm{Fe} / \mathrm{H}]$ is obvious. The observed quantity $[\mathrm{Rb} / \mathrm{Zr}]$ can be converted to a rough neutron density for the $s$ process using the results from Malaney and Lambert (1988) and Malaney (1987) and this is shown in the bottom panel of Fig. 18. The dashed line in the bottom panel is a leastsquares fit to the MS/S, Ba star, and yellow symbiotic points, while the $\omega$ Cen stars (filled squares) clearly fall above this trend. The MS/S, Ba, and symbiotic stars exhibit $s$-process abundance enhancements that result from either the dredge up of processed material in a single star, or mass transfer from a single AGB star (in the case of the $\mathrm{Ba}$ and symbiotic stars). The $\omega$ Cen stars, on the other hand, owe their $s$-process enhancements to the material from which they formed, as first suggested by Lloyd Evans (1983) and shown recently by Vanture et al. (1994). The $s$-process elements in $\omega$ Cen presumably result from processing by a number of AGB stars covering a range in mass and metallicity, as well as $\mathrm{Fe}$ abundances ejected from SN II. It may not be surprising that they do not fall on a relation defined by single AGB stars; clearly $\mathrm{Rb}$ in $\omega$ Cen deserves further attention in the future. Concentrating only on the $s$ process isolated from single AGB stars, the linear least-squares fit from Fig. 18 has a slope of -0.97 , or, $N_{n} \propto N_{\mathrm{Fe}}^{-1}$. This is the expected result from the simple arguments discussed above for the ${ }^{13} \mathrm{C}$ neutron source. If this result holds for a larger sample of stars covering a range in metallicity, it may suggest that the internal structure of the Heburning shells in AGB stars is largely independent of metallicity.

\section{E. Recent models: Radiative burning of ${ }^{13} \mathrm{C}$ during the AGB interpulse phase}

Based upon the observational results provided by the heavy element $s$-process rich stars, there is strong evidence that ${ }^{13} \mathrm{C}(\alpha, n){ }^{16} \mathrm{O}$ is the dominant neutron source in the AGB stars. Both the increasing neutron exposures and neutron densities support the picture of a neutron source whose main reactant is a primary element $\left({ }^{13} \mathrm{C}\right.$ from ${ }^{12} \mathrm{C}$ from ${ }^{4} \mathrm{He}$ ). The production of sufficient quantities of ${ }^{13} \mathrm{C}$ at the $\mathrm{H}-\mathrm{He}$ interface remains a difficult problem for theorists to model, but much progress has been made in recent years in reconciling model predictions from AGB stars with the observations (see Sec. III by I. Iben in this review).

Based on models of intermediate mass stars (say $\left.M \sim 4-8 M_{\odot}\right)$, neutrons were predicted to be generated by the ${ }^{22} \mathrm{Ne}(\alpha, n){ }^{25} \mathrm{Mg}$ reaction (see Iben and Renzini, 1983, for references to these earlier models). In such models, peak neutron densities at solar metallicities reached $10^{9}-10^{10} \mathrm{~cm}^{-3}$; as can be seen from the bottom panel of Fig. 18, such densities are not fit by the $\mathrm{Rb} / \mathrm{Zr}$ abundance ratios observed in solar metallicity AGB stars. Various modifications were tried by a number of authors and Lambert (1991) and Lambert et al. (1995) give nice discussions of these efforts. The basic result is that intermediate mass AGB models with the neon-22 neutron source do not provide the low neutron densities needed for the $s$ process.

Investigations using lower mass AGB models with the ${ }^{13} \mathrm{C}$ neutron source require some form of extra mixing at the $\mathrm{H}-\mathrm{He}$ interface. Using refinements to semiconvective mixing, originally used for Population II AGB models by Iben and Renzini (1982a, 1982b) and Hollowell and Iben $(1988,1989)$, it was found that thermal pulses in such models give rise to a double burst of neutrons. In the first burst, neutrons are generated at $T \sim 10^{8} \mathrm{~K}$ by ${ }^{13} \mathrm{C}(\alpha, n){ }^{16} \mathrm{O}$, while in a second, weaker burst (later in the evolution of the thermal pulse when temperatures are higher) ${ }^{22} \mathrm{Ne}(\alpha, n){ }^{25} \mathrm{Mg}$ is activated, but contributes much less to $s$-process production. Gallino (1989) and Käppeler et al. (1990) explored such models; however, the total amount of ${ }^{13} \mathrm{C}$ found in the He shell prior to ignition was basically an adjustable parameter. Busso et al. $(1992,1995)$ continued such parametrized calculations with the s-process neutron density most strongly dependent on how much ${ }^{13} \mathrm{C}$ is synthesized by $\mathrm{H}$ diffusing into the shell between thermal pulses and the rate of injection of the ${ }^{13} \mathrm{C}$ into the convective He-burning shell. In all cases, however, the predicted neutron densities were still higher than indicated by the observations.

A recent potential breakthrough in understanding the discrepancies between the neutron densities in the AGB models versus the observations has been found by Straniero et al. (1995). These authors followed the evolution of a $3 M_{\odot}$ stellar model from the ZAMS up to thermal pulses on the AGB. They find that any ${ }^{13} \mathrm{C}$ synthesized at the H-He interface on the AGB in between thermal pulses (by any mechanism) is burned via $(\alpha, n)$ reactions before the next thermal pulse develops. This results in two major modifications to the $s$ process: the first is that most of the $s$-process nucleosynthesis occurs at temperatures of less than $10^{8} \mathrm{~K}$. At these lower temperatures, the rate at which ${ }^{13} \mathrm{C}$ burns is much slower, with lower resultant neutron densities of $\sim(1-2) \times 10^{7}$ $\mathrm{cm}^{-3}$ at solar metallicity. Inspection of the lower panel of Fig. 18 shows that this value is in excellent agreement with the observationally derived $N_{n}$ 's. The second important result is that much of the $s$ process will now occur in a radiative, instead of a convective, environment. Because the temperature and density vary throughout the radiative He shell, each radiative layer is characterized by different conditions, such that the resulting distribution of neutron exposures is not an exponential distribution. Further modeling may result in bet- 
ter fits to the abundance distributions in low metallicity $s$-process rich stars which, traditionally, seem to have been better fit by single exposure models (e.g., Vanture, 1992a; Smith et al., 1996, 1997).

\section{THE $r$ PROCESS}

\section{A. Introduction}

Of the eight nucleosynthetic processes described by $\mathrm{B}^{2} \mathrm{FH}$, the theoretical quest to explain the production of the $r$-process isotopes, and in particular to identify a plausible astrophysical site capable of synthesizing them in amounts that satisfy observational constraints, has entertained perhaps the widest range of possibilities. Many of the basic nuclear systematics of rapid neutron capture on preexisting seed nuclei discussed in the first and pioneering explorations of $\mathrm{B}^{2} \mathrm{FH}$ remain true in the modern context. Some of the details have changed, especially in the light of new observations and measurements. For example, the accepted values of the solar $r$ abundances, scarcity of reliable nuclear data in the vicinity of the neutron drip line, and the observed $r$-process abundance levels as a function of the Milky Way's evolutionary history each determine to some extent the important details that the origin site must satisfy. The difficulty is not a lack of theoretical modelsthere are numerous ones and the large number of models is a measure of the complexity of the problembut deciding which one (or ones) are the most promising. The ultimate goal of a successful model for the origin site of the $r$ process must be explaining the variety of stellar observations with the nuclear data that are available, and to provide unambiguous predictions that can be tested by future observations.

$\mathrm{B}^{2} \mathrm{FH}$ and Cameron (1957) suggested that during the $r$ process a large flux of neutrons (whose source was unspecified) would be captured by seed nuclei (typically iron) on time scales that were short compared to the $\beta^{-}$ decay time scales of the newly created nuclei. Neutron capture on nuclei with a given number of protons would proceed to very neutron-rich isotopes until the competition between $\beta^{-}$decay and photodisintegration reactions force the nuclear flows to move onwards to nuclei with a larger number of protons. With a suitable neutron exposure, the $r$ process could proceed to the heaviest elements (thorium and uranium), and achieve a steady $(n, \gamma) \leftrightarrow(\gamma, n)$ flow equilibrium. As the neutron flux diminished, $\beta^{-}$decays would then populate the first stable isobar encountered for a given mass number $A$. Comprehensive reviews of the $r$ process by Hillebrandt (1978), Schramm (1982), Mathews and Ward (1985), Cowan et al. (1991), and Meyer (1994) provide very thorough and complete background material. The purpose of this survey is to confront the search for the astrophysical origin site of the $r$ process with the observational and theoretical information that has accumulated over the last few years.

\section{B. A search for the astrophysical site}

All proposed origin sites for the $r$ process can be classified as either primary or secondary production sites. In the primary production scenarios, neutrons are captured rapidly onto seed nuclei that are made in situ. Notable examples would include inhomogeneous primordial nucleosynthesis, supernova cores, jets and/or magnetic bubbles inside rotating stellar cores, and binary compact remnant interactions. In secondary production scenarios, neutrons are rapidly captured onto seed nuclei made by a previous generation of stars. These would include explosive helium or explosive carbon burning in massive stars, neutrino irradiation in the helium layer of massive stars, helium core flashes in low mass stars, intermediate and low mass stars as they pass through the asymptotic giant branch phase of evolution, accretion disks around neutron stars, and classical novae. The detection of solar $r$-process abundances in the $56 \leqslant Z \leqslant 76$ range in very metal-poor (thus presumably very old) halo stars strongly suggests a primary $r$-process origin site that contributed its nucleosynthetic products to the interstellar medium (e.g., Type II supernovae of Pop III or II) prior to the formation of currently observed halo stars (Sec. VIII). The present observational data for $Z<56$, however, is not so conclusive and could permit a secondary origin site.

Much of the recent theoretical work has centered on core collapse supernovae, especially within the region where a neutrino-driven wind is blown from the surface of a nascent neutron star following a delayed Type II or Ib supernova explosion. As the gravitational binding energy of the neutron star is liberated during its cooling time scale $\left(\tau_{\mathrm{KH}} \sim 10 \mathrm{~s}\right)$, the enormous neutrino flux drives the mass loss and provides unique physical conditions under which neutron-rich heavy element synthesis can occur. The $r$ process is primary in this scenario, and is not a purely neutron driven process. It begins with seed nuclei synthesized through a reassembladge of nucleons from an alpha-rich freeze out from nuclear statistical equilibrium in which there is a large neutron excess. This process has been termed the " $\alpha$ process" (Woosley and Hoffman, 1992; Witti et al., 1994). The same name was originally used by $\mathrm{B}^{2} \mathrm{FH}$ to describe what is presently referred to as "neon burning." The resulting distribution of seed nuclei is centered on the krypton to zirconium region with $A \sim 100$, not on iron peak nuclei (as would be the case for a freeze out with a small neutron excess). The larger neutron excess is due to the intense neutrino flux from the protoneutron star, which drives a large number of neutron liberating charged current neutrino capture reactions $(\nu, n)$ on free nucleons. A freeze out with a large neutron excess bypasses the first closed neutron shell at $N=50$, bridging the waiting point there, and shortens the overall timescale needed for a solar abundance producing $r$-process to operate. During the subsequent neutron capture phase of this $r$-process scenario, the presence of these heavier seed nuclei reduces the overall neutron to heavy seed ratio required of a purely neutron-driven process. 
The total mass of $r$-process material ejected is typically $\sim 10^{-5} M_{\odot}$ per supernova, and is set from first principle calculations of the neutrino wind's physical properties. This value of the mass ejected per supernova is consistent with estimates of the Galactic Type II + Ib supernovae rate (10-1 events per century) and the total mass of $r$-processed material $\left(\simeq 10^{4} M_{\odot}\right)$ inferred to presently exist in the $\simeq 10$ Gyr old Galaxy. That is, neither too little material is ejected, nor too much.

The $r$ process described above that may occur within a given mass element can be succinctly described by three parameters: evolution of the entropy per baryon $S / N_{A} k$ (which specifies the thermodynamics of the material), evolution of the electron fraction $Y_{e}$ (which specifies the composition of the material and is related in a simple algebraic way to the neutron excess), and the dynamic timescale $\left(t_{\mathrm{dyn}}\right)$ over which the material cools. Larger entropies give smaller abundances of heavy seed nuclei. This is due to the large density dependence of the intrinsically weak three-body reaction sequences $\alpha(\alpha \alpha, \gamma){ }^{12} \mathrm{C}$ and $\alpha(\alpha n, \gamma){ }^{9} \mathrm{Be}(\alpha, n){ }^{12} \mathrm{C}$. These two reaction chains are very important because they bridge the unstable mass gaps at $A=5$ and $A=8$. Physically, a high entropy means many photons per baryon. A significant fraction of these photons can be on the high energy tail of the Bose-Einstein distribution, and therefore can maintain, for example, a low abundance of ${ }^{9} \mathrm{Be}$ through the highly efficient photodisintegration reactions. The neutron excess determines the overall availability of neutrons in the material. It chiefly affects the path of the nuclear flow and hence the distribution of heavy seed nuclei produced at the end of the freeze out from nuclear statistical equilibrium. In general, lower values of $Y_{e}$ give smaller abundances of heavy seed nuclei. A shorter expansion time scale also reduces the seed abundance by limiting the time over which the freeze out operates. A smaller heavy seed abundance at the end of the $\alpha$ process gives a larger neutron-to-seed ratio, which enhances the production of heavier $r$-process nuclei. It is important to emphasize that the entropy and neutron excess of a given mass element are time-dependent quantities. The total $r$-process yield is the sum over all ejected mass elements, each of which follows a somewhat different nucleosynthetic pathway as a result of the thermodynamic history and initial neutron excess of each mass element. Hydrodynamics (especially in multiple dimensions) are therefore important in our understanding of neutron-rich heavy element synthesis (Burrows et al.; 1995, Bazan and Arnett, 1994a, 1994b).

\section{Early model results, from conflict to clarity}

Detailed $r$-process nucleosynthesis studies in spherically symmetric $20 M_{\odot}$ stars have been studied by Woosley et al. (1994) and Takahashi et al. (1994). In both works a proper amount of $r$-process material was ejected, a reasonable agreement with the solar $r$-process isotopic distribution was achieved, and high entropies $\left(S / N_{A} k \sim 400\right)$ were considered to be required to reproduce the solar abundance of platinum peak $(A=195)$ nuclei. Both works also shared the common drawback of producing unacceptably large amounts of the $N=50$ closed neutron shell nuclei ${ }^{88} \mathrm{Sr},{ }^{89} \mathrm{Y}$, and ${ }^{90} \mathrm{Zr}$ during the $\alpha$-process phase of their calculations. This was caused by having too many neutrons present $\left(Y_{e} \sim 0.46\right)$ during the early evolution of the neutrino-powered wind. If the number of neutrons was decreased slightly ( $Y_{e} \sim 0.48$ ), the production factors for the $N=50$ closed neutron shell nuclei became smaller, and hence more palatable (Hoffman et al., 1996). In this case, some of the light $p$-process nuclei were coproduced, most notably the hitherto unproduced isotope ${ }^{92} \mathrm{Mo}$ (Sec. XIV). Several correlations have been noted between the production ratios of some light- $p$ and $r$-process isotopes as calculated by these studies and the production ratios that have been measured in the Allende and Touluca meteorites. This is suggestive, but certainly not conclusive, evidence that such isotopes were produced in a common environment. The isotope ${ }^{64} \mathrm{Zn}$ is also produced, and appears to be a unique signature of the $\alpha$ process. This might be important for understanding the observed zinc to iron $[\mathrm{Zn} / \mathrm{Fe}]$ ratio in Galactic dwarf stars and the QSO absorption line systems, which show a solar $[\mathrm{Zn} / \mathrm{Fe}]$ ratio for all metallicities larger than $0.001 Z_{\odot}$.

Each group shared the dubious distinction of claiming significantly different entropy per baryon structures in the neutrino-powered wind. This spurred a quest for a more physical understanding of the conditions extant in these winds. Inspired by the early work of Duncan, Shapiro, and Wasserman (1986) analytic equations for the entropy per baryon, dynamic time scale, and mass-loss rate were derived as functions of the neutron-star mass, radius, and emergent neutrino luminosities and spectra (Qian and Woosley, 1996). An equation for the electron fraction in terms of the charged current lepton capture reactions on free nucleons was also derived. These analytical expressions, with the transparent physical insight their derivations provide, were verified to a high degree of accuracy by resolved numerical simulations of steadystate neutrino winds. For typical neutron star properties, neutrino luminosities and spectra, the derived entropy per baryon fell short, by roughly a factor of 2 , of the value apparently needed to produce the $A=195$ platinum peak $r$-process nuclei (Hoffman et al., 1997). When a modest increase to the energy produced by the wind was added at the base of the neutrino atmosphere, sufficient conditions for producing platinum peak nuclei were achieved. Possible sources of energy capable of justifying this energy perturbation, which were not included in either the analytical equations or the numerical simulations, could include rotation or vibration of the neutron star, general relativistic effects, and magnetic fields (Qian and Woosley, 1996).

It is important to stress that high entropy is not a necessary requirement for a successful neutrino-wind driven $r$ process (Fig. 19; see also Hoffman et al., 1997). A shorter dynamic time scale will limit the duration of the precursor $\alpha$ process. This reduces the heavy seed abundances and increases the neutron to heavy seed ra- 


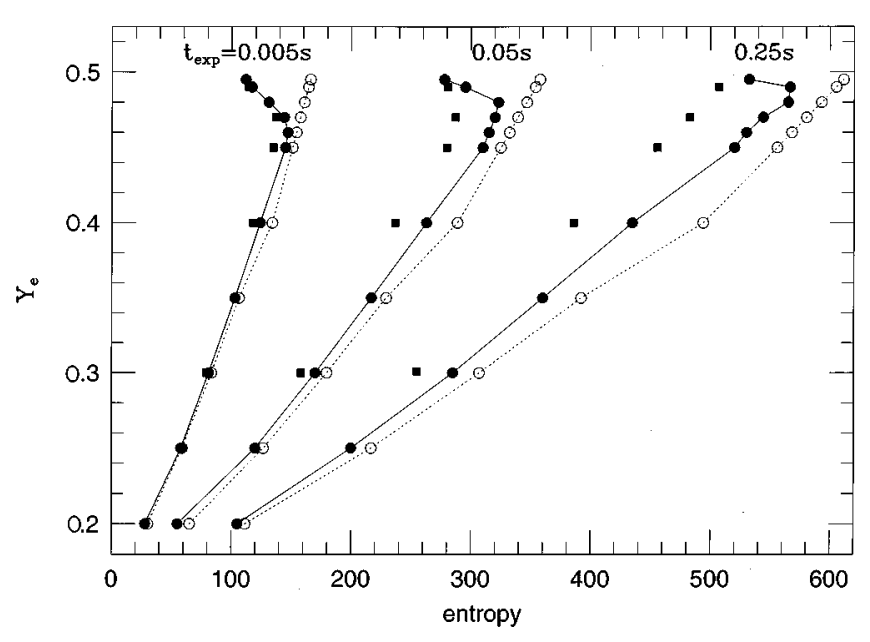

FIG. 19. The minimum entropy required to make platinum peak nuclei $(A \sim 195)$ by neutron capture following an $\alpha$ process for a given electron fraction $\left(Y_{e}\right)$ and expansion time scale $\left(t_{\text {exp }}\right)$. Filled circles connected by solid lines are the results of a numerical calculation for a constant entropy of $S=3.33 T_{9}^{3} / \rho_{5}$. Filled squares (not connected by a line) are the results of a numerical calculation for an exact adiabatic equation of state. Open circles connected by dotted lines are from analytic expressions for the minimum entropy. Figure from Hoffman et al. (1997).

tio for the subsequent neutron capture phase. The same neutron to seed ratio can be obtained at $\frac{1}{2}$ the entropy if the expansion time scale is about 8 times shorter. The chief utility of such parametrized reaction network surveys could be to determine the suitability of detailed core collapse simulations (or other astrophysical environments, e.g., Ruffert et al., 1997) as potential $r$-process sites, provided the $r$ process is envisioned as coming from a neutron-rich, $\alpha$-rich freezeout from nuclear statistical equilibrium.

\section{Twisting in the wind}

As with any evolving theory, our understanding of the $r$ process in neutrino winds is far from complete, and new twists to our current thinking about the time evolution of the key parameters that describe it continue to permeate the literature. At issue are uncertainties in the nuclear equation of state, neutrino-nucleus interaction cross sections, possible neutrino-flavor transformations, fallback of freshly synthesized material onto the compact remnant, and neutrino transport. Their continuing resolution will play a central role in determining whether the neutrino-driven wind will remain an attractive site for the $r$ process.

Neutrino transport is probably the most serious, and perhaps the most challenging, of these issues. The transport of neutrinos directly impacts the nucleosynthesis through the time evolution of $Y_{e}$; previous studies suggest an almost uncomfortable sensitivity of the nucleosynthesis to this parameter (Sec. XIV). $Y_{e}$ also couples to the hydrodynamical question of how much mass falls back onto the remnant. Even if all supernovae core- collapse events can make a successful $r$ process, much (or all) of it might not be injected into the interstellar medium. Neutrino transport also affects the magnitude of any asymmetrical energy deposition (kicks), which may manifest itself in the observed pulsar proper motions (Burrows and Hayes, 1996). Studies to date have generally relied upon flux-limited multigroup neutrino diffusion schemes. A less ambiguous, if more ambitious, treatment would solve the Boltzmann equation for all the neutrino species (Mezzacappa and Bruenn, 1993) and couple it to a multidimensional hydrodynamics solver. Such tools are just now coming to the forefront of supernova research.

Neutrino-nucleus interactions during the early phases of the core collapse are probably essential, and were not included in the $r$-process studies cited above. The electron fraction is set by a competition between chargedcurrent lepton $\left(e^{-}, e^{+}, \nu_{e}\right.$, and $\left.\overline{\nu_{e}}\right)$ capture processes on free nucleons, the neutrino capture reactions being the most important. Neutral current spallation of $\alpha$ particles is also important, but only in the limit of a high entropy $r$ process. Neutrino interactions with heavy nuclei do not affect $Y_{e}$ (McLaughlin et al., 1996; Hoffman et al., 1997), however, they do exhibit interesting effects during the neutron-capture phase by spreading and damping the peaks in the abundance distribution. Haxton et al. (1997) find that nuclei in the mass regions $124 \leqslant A \leqslant 126$ and $183 \leqslant A \leqslant 187$ show an inordinate sensitivity to neutrino postprocessing effects, which could impose stringent bounds on the freeze-out radii and dynamic time scales governing the $r$ process in future core collapse models. Freeze-out from statistical equilibrium has already been deemed necessary to account for other unique isotopic signatures within the solar $r$-process abundance pattern using $\beta$-decay properties and abundances of the $r$-process nuclei themselves (also see Kratz et al. 1993).

The evolution of $Y_{e}$ might also be affected by neutrino flavor transformation near the surface of the nascent neutron star due to the Mikeheyev-SmirnovWolfenstein (MSW) effect (Fuller et al., 1987). Transformation between $\bar{\nu}_{\mu(\tau)}$ and $\bar{\nu}_{e}$ can occur if the vacuum masses for these species satisfy $m_{\bar{\nu}_{e}}>m_{\overline{\nu_{\mu, \tau}}}$ and $\Delta m^{2}>1 \mathrm{eV}^{2}$. Such transformations, however, are not expected to drive $Y_{e}>0.5$, and hence do not pose any difficulty for the envisioned $r$-process origin site. In stark contrast, $\nu_{\mu \tau}$ to $\nu_{e}$ conversions, in which the neutrino energy hierarchy of the $\nu_{e}$ is reversed, could have damaging consequences (Qian and Fuller, 1995).

\section{E. Concluding remarks}

The search for an overarching $r$-process paradigm that encompasses the measured solar $r$-process abundances, observed halo and disk star $r$-process abundances, calculated $r$-process nucleosynthesis, and observed chemical evolution of the $r$-process abundances has not been without its sucesses, but it remains unfulfilled. The attempt to satisfy these demands with a natu- 


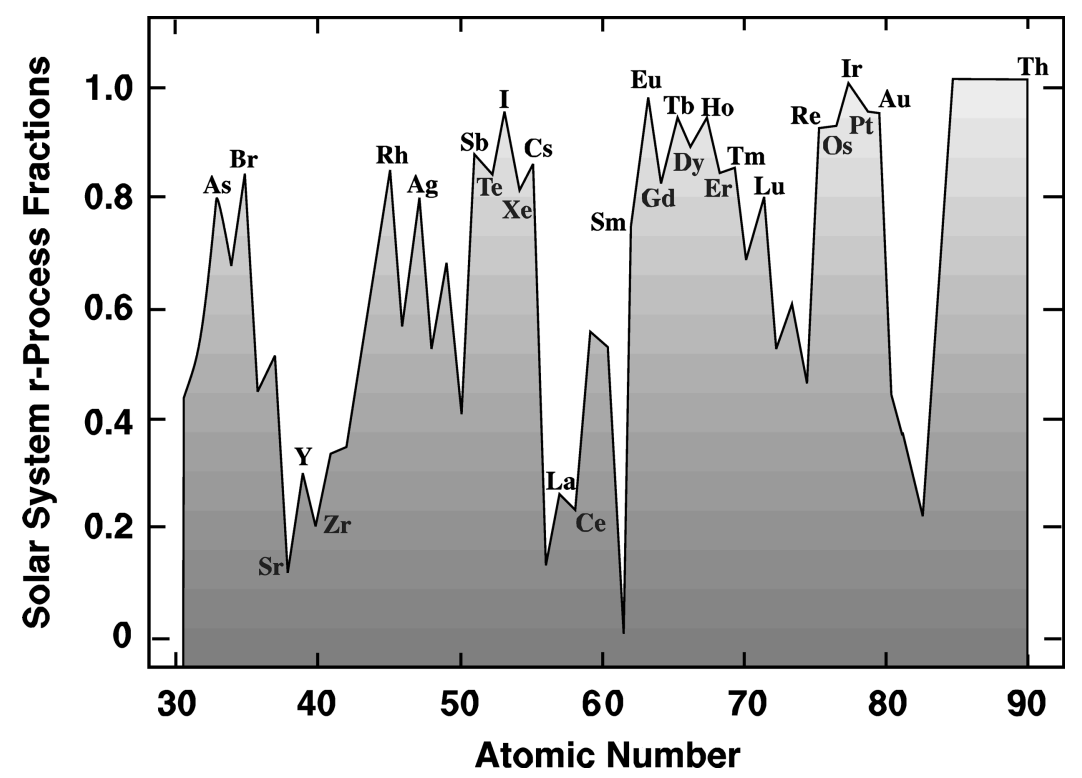

FIG. 20. Fractions of neutron-capture solar system element abundances attributable to the $r$ process $\left(F_{\odot}[r]\right)$ as a function of atomic number. These abundance fractions have been determined by Sneden et al. (1996) and Cowan and Burris (1997) by combining $r$ - and $s$-process solar system isotopic fractions (Käppeler et al., 1989) with solar system elemental abundances (Anders and Grevesse, 1989; revised abundance recommendations of Grevesse et al., 1996, for a few elements would not materially affect these fractions).

ral astrophysical origin site encompassing these aspects promises to be an exciting adventure into the next millennia.

\section{OBSERVATIONS OF THE $r$ PROCESS}

\section{A. Defining The $r$-process elements}

In this section we will review the stellar spectroscopic data that confirm the existence of $r$-process nucleosynthesis at the early epochs of our galaxy, and that show the growth of the $r$-process element abundances with time. Only analyses of cool stars will be considered, in order to avoid the spectroscopic peculiarities of hot stars that are more indicative of surface and outer envelope anomalies rather than true element nucleosynthesis. Stellar metallicity will of necessity be the stand-in for stellar age, with the most metal-poor stars being assumed to be the oldest stars. Much of the discussion will center on $r$-process elements in old, very metal-poor stars of the Galactic halo, for $r$-process elements can be most easily disentangled from other elements in such objects. Although we will concentrate on positive evidence for $r$-process nucleosynthesis, we will also comment on the relative contributions of $r$ and $s$ processes as a function of stellar metallicity.

$\mathrm{B}^{2} \mathrm{FH}$ devoted nearly 20 pages to a detailed description of physical conditions for and expected yields from the $r$ process. However, their discussion of direct proof of the existence of the $r$ process in stars was limited to a possible interpretation of Type I SNe light curves as being due to the decay of ${ }^{254} \mathrm{Cf}$ (which later turned out to be incorrect). There is still no direct evidence of $r$-process species in the spectra of SNs or their remnants
(Wallerstein et al., 1995). While $\mathrm{B}^{2} \mathrm{FH}$ could display spectra of stars with very obvious enhanced $s$-process compositions (their Plate 3), no such dramatic examples of stars with enhanced $r$-process compositions were known for a very long time after publication of $\mathrm{B}^{2} \mathrm{FH}$.

The firm identification of $r$-process nucleosynthesis products in stars was difficult, because $r$-process elements are not easy to observe in cool stars. To amplify this point we first identify all elements whose abundances are sensitive to $r$-process synthesis, remembering that stellar spectroscopy is mostly limited to determination of elemental, and not isotopic abundances. In Fig. 20 we display the solar system elemental abundance fractions attributable to the $r$ process $\left(F_{\odot}[r]\right)$ and the $s$ process $\left(F_{\odot}[s]\right)$ of all stable "neutron-capture" elements in the range $31 \leqslant Z \leqslant 83$. We also show the solar system abundance fraction of the important unstable element Th $(Z=90)$, which is produced only in the $r$ process $\left(F_{\odot}[r]=1.0\right)$ because $s$-process nucleosynthesis cannot proceed past $Z=83$. The values of $F_{\odot}[r]$ and $F_{\odot}[s]$ have been determined by Cowan and Burris (1997) in the manner described by Sneden et al. (1996); see Fig. 20 caption for details of this procedure. In this figure, element symbols are printed near the $F_{\odot}[r]$ values, but only for those elements whose solar abundance is due chiefly to the $r$ process (arbitrarily defined here as $\left.F_{\odot}[r]>0.75\right)$ or the $s$ process $\left(F_{\odot}[r]<0.25\right)$. Therefore for convenience we shall call the labeled elements $\mathrm{Sr}$, $\mathrm{Zr}, \mathrm{Ba}, \mathrm{Ce}, \mathrm{La}$, and $\mathrm{Pb}$ " $s$-process elements," and the remaining labeled elements will be called the " $r$-process elements." It must be acknowledged that the neutroncapture element abundances in a given star may be due to a very different mix of $r$ - and $s$-process syntheses than those that produced the solar system composition. 
There are $24 r$-process elements identified in Fig. 20, but unfortunately only a handful of these elements are easily observable in stars. Several $r$-process elements (As, $\mathrm{Br}, \mathrm{Te}, \mathrm{I}, \mathrm{Xe}, \mathrm{Cs}, \mathrm{Re}$ ) have never been detected in the solar photospheric spectrum (e.g., Grevesse et al., 1996). Several other $r$-process elements (Ag, Lu, Os, Ir, $\mathrm{Pt}, \mathrm{Au}$ ) display only one or two usable solar spectral features, and then only in the crowded and difficult to observe UV spectral region $(\lambda<3500 \AA)$. Thus the "observable" $r$-process elements usually come down to those in the range $62 \leqslant Z \leqslant 69$, and of those elements only $\mathrm{Eu}\left(Z=63, F_{\odot}[r] \simeq 0.97\right)$ has spectral features strong enough to be detected in many very metal-poor stars.

\section{B. Early $r$-process discoveries}

Most stellar $r$-process surveys have concentrated on $\mathrm{Eu}$, and assessments of the $r$ - and $s$-processes contributions to stellar compositions usually compare Eu abundances to those of $\mathrm{Ba}(Z=56)$, a nearby $s$-process element $\left(F_{\odot}[s] \simeq 0.88\right)$ with strong spectral features. To begin a brief history of $r$-process observations, Wallerstein et al. (1963) detected a single Eu II feature in their spectrum of HD 122563, which is by far the brightest very metal-poor star $([\mathrm{Fe} / \mathrm{H}] \sim-2.7)$ in the sky. Their analysis, and Pagel's (1965) reanalysis of the same data set, did not find an easy match to $r$-process predictions, for they noted that $\mathrm{Eu}$ was deficient with respect to $\mathrm{Fe}$ in this star: $[\mathrm{Eu} / \mathrm{Fe}] \sim-0.4$. However, the $\mathrm{Ba}$ abundance of this star was found to be even lower: $[\mathrm{Ba} / \mathrm{Fe}] \sim-1.0$, leading Pagel to note that the relative abundances of neutron capture elements in HD 122563 were "an almost exact mirror-image" of the abundance enhancements of $\mathrm{CH}$ stars. Therefore, since the neutron-capture element enhancements of $\mathrm{CH}$ stars are due to the $s$ process (much greater overabundances of $\mathrm{Ba}, \mathrm{La}$, and $\mathrm{Ce}$ than $\mathrm{Eu}$ ), this statement then constitutes the first solid evidence for $r$-process contributions to the material of very old, very metal-poor stars.

A pioneering survey of neutron-capture elements in many metal-poor stars by Spite and Spite (1978) showed a steep decline in relative $\mathrm{Ba}$ abundances with decreasing metallicity (that is, $[\mathrm{Ba} / \mathrm{Fe}] \propto[\mathrm{Fe} / \mathrm{H}]$ ) for stars in the metallicity range $-1.3 \leqslant[\mathrm{Fe} / \mathrm{H}] \leqslant-2.6$, while finding little change in the relative Eu abundances $([\mathrm{Eu} / \mathrm{Fe}] \sim 0$ at all metallicities). Consideration of these abundance ratios, along with those of lighter neutron-capture elements $\mathrm{Sr}$, Y, and Zr led Truran (1981) to claim that "the heavy element abundance patterns characteristic of the most iron-deficient stars ... are therefore entirely compatible with their having an $r$-process origin."

Sneden and Parthasarathy (1983) investigated the HD 122563 spectrum over greater spectral range and with higher signal-to-noise data, producing first detections of several $r$-process elements (Gd, Dy, Er) in a very metalpoor star. The relative underabundance of all neutroncapture elements in this star was confirmed, but the element-to-element abundance pattern could only be matched by a dominant $r$-process contribution to the element distribution.

Soon it became apparent that some very metal-poor stars possess neutron-capture element overabundances, most notably HD 115444 (Griffin et al., 1982), and HD 110184 (Sneden and Pilachowski, 1985). The large overabundances of $\mathrm{Eu}$ in both of these stars signaled a strong $r$-process contribution. Finally, a 20 -star survey by Gilroy et al. (1988) demonstrated the pervasiveness of $r$-process nucleosynthesis products in metal-poor stars, and argued strongly for significant star-to-star scatter in the overall levels of the neutron-capture element abundances relative to $\mathrm{Fe}$ for stars with $[\mathrm{Fe} / \mathrm{H}]<-2$. Gilroy et al. suggested that significant contributions by $s$-process nucleosynthesis to the neutron-capture elements are only apparent in stars more metal-rich than $[\mathrm{Fe} / \mathrm{H}] \sim-2$. Results from other investigations of neutron-capture elements from this era may be found in reviews such as Wheeler et al. (1989).

\section{Recent $r$-process surveys}

Emphasis in recent years has turned to large surveys of stars over a wide range of metallicity. These surveys try to elucidate abundance ratio trends that might be apparent even though the ratios for individual stars may still have substantial uncertainties. Major boosts to these studies have come from new catalogs of low metallicity stars. To cite two examples, Laird et al. (1988) have produced a large set of $[\mathrm{Fe} / \mathrm{H}]$ values for nearby (mostly low luminosity dwarf and subgiant) high proper motion stars, and Beers et al. $(1985,1992)$ have an ongoing program to discover extremely low metallicity, high luminosity giant stars at large distances into the galactic halo.

These metallicity surveys are proving especially useful in finding stars with $[\mathrm{Fe} / \mathrm{H}]<-2$; such stars surely were the recipients of the first wave of galactic nucleosynthesis, and abundance ratios of many element groups with respect to Fe show major departures from solar ratios in such stars. In Fig. 21 we show average abundance ratios of three neutron-capture element groups from some recent studies of low metallicity stars. Since the purpose of this figure is only to show rough abundance ratio trends, several stipulations accompany this figure. First, the data chosen for inclusion here are representative (emphasizing large-sample abundance ratio surveys), and are not meant to include all studies of low metallicity stars. Second, no attempt has been made to renormalize the data sets of different studies to a common scale (see the individual cited papers for discussions of this point). Third, some bright "standard" stars (e.g., HD 122563) appear in more than one survey, but we have chosen to plot all the points from each survey. Fourth, we have made these three-element averages in an attempt to minimize analysis uncertainties; in most studies often only one or two lines of a given element have been analyzed, and there are difficulties in deriving accurate abundances from the very strong spectral features of $\mathrm{Sr}$ 


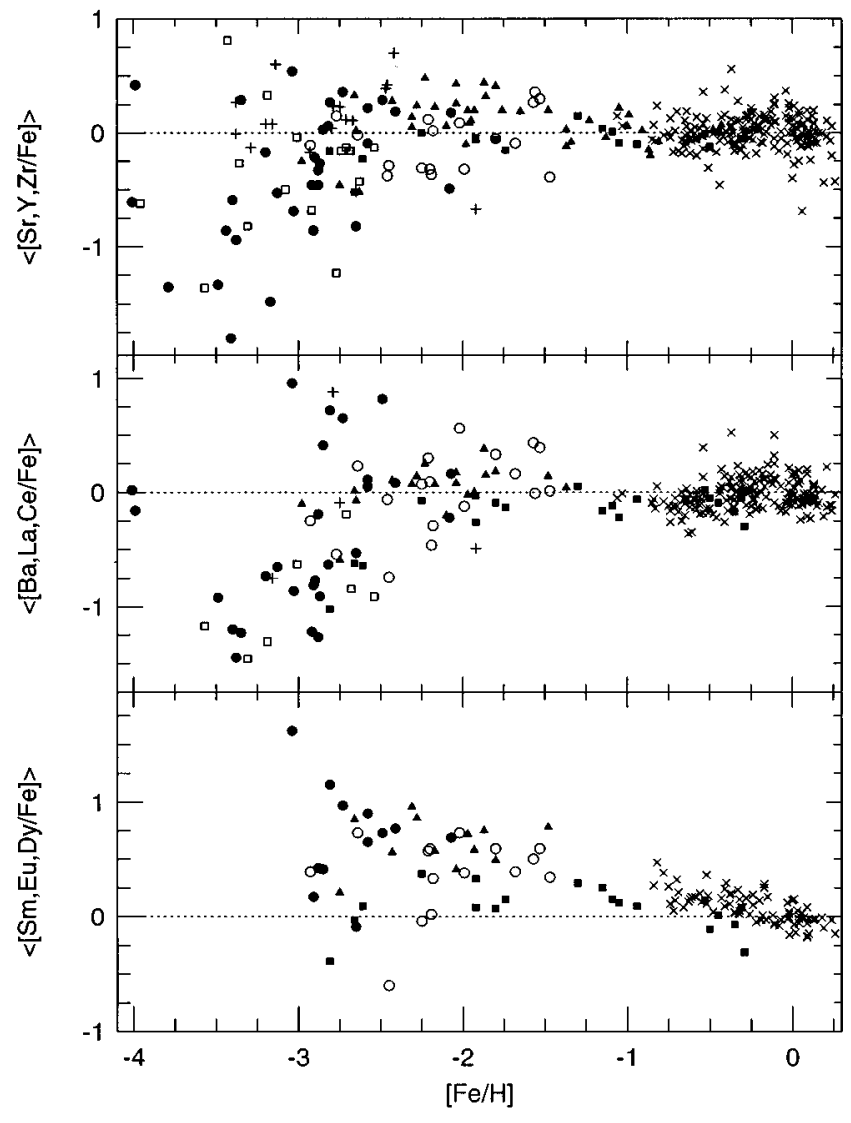

FIG. 21. Mean relative abundance ratios of "light" $s$-process elements Sr, Y, and Zr (top panel), "heavy" $s$-process elements $\mathrm{Ba}, \mathrm{La}$, and $\mathrm{Ce}$ (middle panel), and $r$-process elements $\mathrm{Sm}, \mathrm{Eu}$, and Dy (bottom panel), as functions of stellar metallicity $[\mathrm{Fe} / \mathrm{H}]$. The relative abundances are defined as $[A / B] \equiv \log _{10}\left(N_{A} / N_{B}\right)_{\text {star }}-\log _{10}\left(N_{A} / N_{B}\right)_{\odot}$ for elements $A$ and $B$. In each panel the dotted horizontal lines represent the solar abundance ratios of these elements: filled circles, McWilliam et al. (1995); open circles, Gilroy et al. (1988); open squares, Ryan et al. (1996); plus signs, Ryan et al. (1992); filled circles, Gratton and Sneden (1994); filled triangles, Magain (1989), Zhao and Magain (1990, 1991); crosses, Edvardsson et al. (1993) in the top and middle panels, Woolf et al. (1995) in the lower panel.

II (e.g., Gratton and Sneden, 1994) and Ba II (e.g., Magain, 1995, Sneden et al., 1996). In many stars only a $\mathrm{Sr}$ or a $\mathrm{Ba}$ abundance has been determined, and those abundances must of necessity stand for the threeelement averages in such cases. Finally, we have eliminated some stars that are chemically evolved (e.g., $\mathrm{CH}$ stars) and thus are unrepresentative of the chemical mix from which they formed.

Even with all the preceding caveats, several abundance trends are apparent in Fig. 21. If we arbitrarily label stars with $[\mathrm{Fe} / \mathrm{H}]<-2.5$ as "ultra-metal-poor" (hereafter UMP) stars, at first glance the dominant abundance signature for UMP stars is an extraordinarily large star-to-star scatter in the overall level of neutroncapture elements with respect to $\mathrm{Fe}$. This abundance scatter, which, in the case of the Ba-La-Ce group, is more than a factor of 100 at a given $[\mathrm{Fe} / \mathrm{H}]$ and is well beyond any possible observational and analytical uncertainties. The reality of the star-to-star scatter can be demonstrated with simple comparative plots of the stellar spectra (e.g., Gilroy et al., 1988, McWilliam et al., 1995). Clearly, knowledge of a UMP star's Fe metallicity gives one absolutely no insight to its neutron-capture element abundance levels. More importantly, this very large scatter means that the neutron-capture element abundances in UMP stars are products of one or very few prior nucleosynthesis event(s) that occurred in the very early, poorly mixed galactic halo, an interpretation favored by Truran (1981) from different observational clues.

Beyond the obvious star-to-star scatter, the data displayed in the top panel of Fig. 21 show that the light $s$-process elements on average are nearly as abundant with respect to Fe in UMP stars as they are in the sun. However, there is a pronounced decrease in the $\mathrm{Ba}$ La-Ce group level in most UMP stars (see the middle panel of Fig. 21). Like the observed star-to-star abundance scatter, the observed decline in the Ba-La-Ce group with metallicity is far too large to blame on observational and analytical uncertainties. In contrast to the heavy $s$-process element decline, the $r$-process $\mathrm{Sm}$ Eu-Dy element group remains quite abundant in UMP stars (see the bottom panel of Fig. 21), at least to metallicities as low as $[\mathrm{Fe} / \mathrm{H}] \sim-3$. In many UMP stars, $\langle[\mathrm{Ba}, \mathrm{La}, \mathrm{Ce}]\rangle /\langle[\mathrm{Sm}, \mathrm{Eu}, \mathrm{Dy}]\rangle \sim-0.6$, while for higher metallicity halo stars the average abundance ratio of these two element groups is $\sim 0$. It is tempting to argue, as did Gilroy et al. (1988), that the Ba-La-Ce deficiency argues against significant $s$-process contributions to galactic nucleosynthesis until the halo ISM metallicity attained $[\mathrm{Fe} / \mathrm{H}] \sim-2$. Then, if one adopts the common assumption that significant $s$-process nucleosynthesis occurs mainly during the He-burning phases of intermediate to low mass stars $\left(M<10 M_{\odot}\right)$, the time scale for buildup of galactic halo metallicity to $[\mathrm{Fe} / \mathrm{H}] \sim-2$ must have been no less than $10^{7}$ years (the rough time scale for the evolution of a $10 M_{\odot}$ star).

How dominant is the $r$ process in UMP star neutroncapture elements? The bottom panel of Fig. 21 clearly shows the presence of a vigorous synthesis of the standard $r$-process Sm-Eu-Dy element group early in the history of the galaxy. But was it also responsible for the low level of the element groups that are $s$-process products in solar system material? The nearly solar abundance ratios of the light $s$-process groups, Sr-Y-Zr, argues for significant contributions by the $s$ process in many UMP stars. Moreover, attempts to match a scaled set of $r$-process abundance fractions to the detailed abundance ratios among these elements have not met with much success (e.g., Gilroy et al., 1988, Cowan et al., 1995).

Among the heavier $s$-process elements, Magain (1995) has estimated isotopic ratios of Ba from analysis of a $\mathrm{Ba}$ II line profile in one UMP dwarf star (HD 140283), concluding that no clear evidence exists for a significantly nonsolar, enhanced $r$ - to $s$-process ratio in the total $\mathrm{Ba}$ abundance of this star. On the other hand, 


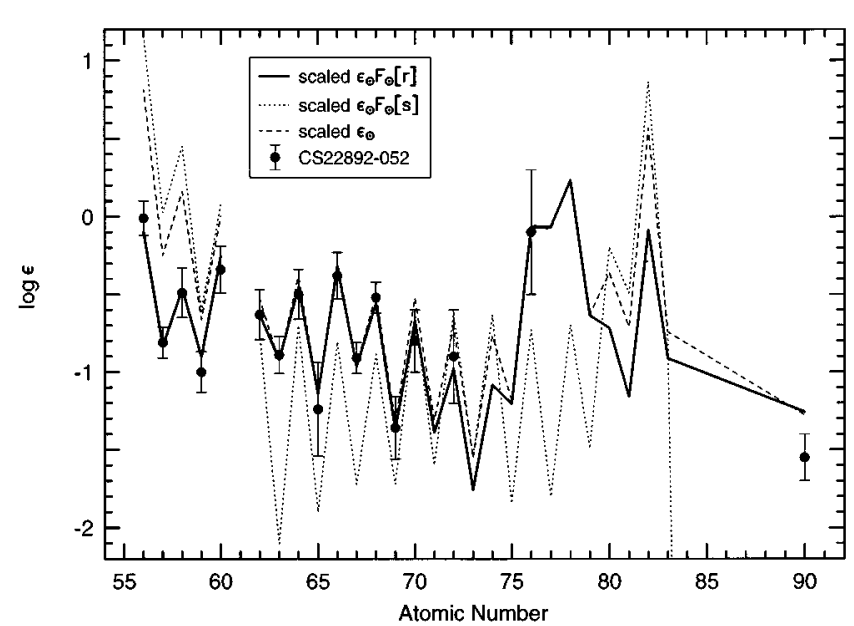

FIG. 22. Neutron capture element abundances in the UMP star CS 22892-052 (filled circles with error bars), compared with scaled solar system abundance distributions. The abundances are "absolute," defined as $\log \epsilon(\mathrm{A})$ $\equiv \log _{10}\left(N_{A} / N_{H}\right)+12.0$ for any element $A$. Anders and Grevesse (1989) and Grevesse et al. (1996) are the sources for the solar system $\epsilon$ values, and the $r$ - and $s$-process curves are generated by multiplying these values by the appropriate $F_{\odot}[r]$ and $F_{\odot}[s]$ fractions discussed earlier in this section. Each solar $\log \epsilon$ curve has been shifted by a single vertical additive constant to match the CS 22892-052 data points. The $r$-process curve has been shifted to make the best average match to the abundances in the range $56 \leqslant Z \leqslant 72$. The total solar system curve has been normalized to agree with the observed $\mathrm{Eu}$ abundance. The $s$-process solar system curve has been shifted by an approximate amount, for it does not match any set of observed abundance ratios.

McWilliam et al. (1995) use the subsolar Ba/Eu ratios of nearly all UMP stars of their sample to assert that early galactic synthesis of $\mathrm{Ba}$ was carried out via the $r$ process. Moreover, serendipitous inclusion of the star CS 22892052 in the McWilliam et al. survey provides perhaps the strongest case yet uncovered of $r$-process dominance in early galactic nucleosynthesis.

CS 22892-052 is a UMP giant star with extraordinary overabundances of all neutron capture elements. It has the largest $r$-process/Fe abundance contrast of any known star: In the bottom panel of Fig. 21, this star appears at $[\mathrm{Fe} / \mathrm{H}] \sim-3.1,\langle[\mathrm{Sm}, \mathrm{Eu}, \mathrm{Dy} / \mathrm{Fe}]\rangle \sim+1.5$. Sneden et al. (1996) have presented a detailed neutron capture element analysis of CS 22892-052 using new high signal-to-noise spectra. In Fig. 22 we display their derived abundances for elements in the range $56 \leqslant Z \leqslant$ 90. Among the 17 elements detected in this star are several (Tb, Ho, Tm, Hf) never before found in cool star spectra. Additionally, the abundances of elements previously detected in other metal-poor stars are based on larger numbers of transitions (18 features in the case of $\mathrm{Nd}$ ) in CS 22892-052, increasing confidence in the derived abundances. Clearly CS 22892-052 is one of the best stellar "laboratories" for investigation of early galactic neutron capture nucleosynthesis. In Fig. 22 we compare the observed CS 22892-052 abundances to scaled solar-system $r$ process, $s$ process, and total abun- dance distributions [see Sneden et al. (1996) for details of this procedure]. Neglecting for the moment the element Th $(Z=90)$, the data match the $r$-process curve exceedingly well, and fail substantially when compared to the other curves (the scaled total solar system abundance curve does indeed match the elements in the range $62 \leqslant Z \leqslant 72$, but that is because these are $r$-process elements in the solar system). There is little reason to postulate a significant $s$-process component to any of these elements in this star. Since the $r$ process apparently cannot occur during quiescent stellar evolution, these neutron-capture species must have been created in a previous stellar generation, almost certainly during the explosive death of a prior high mass star. Additionally, the extreme overabundance of all these elements with respect to Fe strongly suggests that they were created by a single prior supernova that was "local" to the ISM from which CS 22892-052 was created. Finally, it seems remarkable that a scaled solar system $r$-process abundance distribution should match that of an old, UMP halo star so well (Truran, 1996). This seems to imply a universality to $r$-process nucleosynthesis: the conditions in supernovae that lead to production of $r$-process elements that subsequently survive to seed the ISM apparently are always quite similar. Perhaps there is only a relatively narrow mass range of supernovae that contribute to bulk production of $r$-process elements in the galaxy.

\section{Thorium and the age of the halo and disk}

Thorium $(Z=90)$ is synthesized only in the $r$ process and it decays with a half-life of $14 \mathrm{Gyr}$, and so a substantial fraction of any $\mathrm{Th}$ created at any galactic epoch should still survive today. For this reason much effort has been devoted to the derivation of $\mathrm{Th}$ abundances (from the single available Th II spectral feature at 4019 $\AA$ ) and their use in estimations of the age of the galaxy. Butcher (1987) pioneered stellar Th cosmochronology, estimating the age of the galactic disk by comparing Th abundances to those of the stable neutron capture element Nd. His work has been further refined by Morell et al. (1992). Unfortunately, interpretation of Th/Nd ratios for cosmochronology is clouded by the necessity to consider only that part of the $\mathrm{Nd}$ abundance in a star that is due to the $r$ process (a bare majority in the sun: $\left.F_{\odot}[r]=0.53\right)$. Therefore, Pagel (1989) suggested using $\mathrm{Th} / \mathrm{Eu}$ ratios instead, since $\mathrm{Eu}$ is an almost pure $r$-process element $\left(F_{\odot}[r]=0.97\right)$. François et al. (1993) carried out an extensive study of $\mathrm{Th} / \mathrm{Eu}$ in low metallicity halo stars. They found approximately solar abundance ratios of these elements at all metallicities, but they did not derive galactic ages from their data because they felt that the production ratios for these two elements were insufficiently understood to make meaningful age estimates. Frustratingly, in stellar Th cosmochronology one cannot perform the classical parent/daughter test to discover the original content of the parent (which of course must be known to determine a radioactive age for the star). 
CS 22892-052 and other UMP stars that are very rich in $r$-process elements strengthen the use of Th in cosmochronometry (Wallerstein, 1994). The key is the determination of many stable $r$-process abundances (more than just $\mathrm{Eu}$ ), particularly those with atomic numbers as close to Th as possible. Notice in Fig. 22 what little flexibility in the scaled solar system $r$-process distribution is permitted from the 16 stable elements observed in CS 22892-052. This makes plausible the assumption used by Sneden et al. (1996) that the implied, scaled Th abundance (corrected back to the start of the solar system) from the scaled $r$-process curve may be a good representation to the original Th in CS 22892-052. Sneden et al. used the mismatch between predicted and observed Th abundances in this star to suggest that the progenitor supernova to this star exploded 15.2 $\pm 3.7 \mathrm{Gyr}$ ago. Cowan et al. (1997) have considered uncertainties in all aspects of Th observations, nuclear reaction rates, and galactic chemical evolution to suggest that the "age" of the $r$-process elements in CS 22892-052 might be as old as $17 \mathrm{Gyr}$, but ages much younger than about $12 \mathrm{Gyr}$ are unlikely.

\section{E. Filling out the picture}

$r$-process observations have progressed from nonexistent at the writing of $\mathrm{B}^{2} \mathrm{FH}$ to the rich data sets that are available today. However, many years of effort remain to complete our knowledge of these elements and how they relate to galactic nucleosynthesis. Here we highlight a few areas of special interest.

Light neutron capture element synthesis. The unsatisfactory understanding of the Sr-Y-Zr element abundances has been mentioned above. The large abundances of these elements even in stars with very deficient heavier $s$-process elements (the Ba-La-Ce group) may mean that the $s$ process did produce the lightest neutron capture elements during the quiescent evolutionary stages of the high mass stars that eventually manufactured the $r$-process elements at the supernova stage. Alternatively, the $r$ process may have at least two components, perhaps produced in supernovae of different mass. A "second" $r$ process that would produce just the lighter neutron capture elements has been suggested by Wasserburg et al. (1995), but definitive observational tests will be difficult. One possibility might be to search for spectroscopic features of elements in the atomic number range $41 \leqslant Z \leqslant 55$, none of which have been studied extensively in stars other than the sun. Most elements in this range cannot be detected spectroscopically. One possibility is to search for $\operatorname{Ag}(Z=47)$, which has two strong lines in the near-UV solar spectrum. Even this one element filling in a very large neutron capture element gap would allow us to eliminate some of the possible scenarios to explain the lighter neutron capture elements. At present we lack a definitive observational signature to understand the abundances of lighter neutron capture elements in UMP stars.

Detection of the heaviest stable neutron capture elements. If we cannot observe radioactive Th decay prod- ucts, then at least we must derive abundances of several of the heaviest stable elements in UMP stars. This is necessary so that we may predict with more confidence the original Th abundances ejected by supernovae and now residing in diminished numbers in stars with detected Th features. In practice, the elements Os, Ir, Pt, $\mathrm{Au}$, and $\mathrm{Pb}$ are candidates for observation, but all strong transitions of these elements occur in the UV below the atmospheric cutoff. Therefore the high resolution spectrographs of the Hubble Space Telescope must be employed for this work, and Cowan et al. (1996) report detections of $\mathrm{Os}, \mathrm{Pt}$, and $\mathrm{Pb}$ in the moderately metal-poor HD $126587([\mathrm{Fe} / \mathrm{H}] \sim-1.7)$. The derived abundances for these elements are consistent with a scaled, mixed $r$ - and $s$-process solar abundance distribution of elements from $Z=56$ to $Z=82$. In new HST observations, this same group has detected Pt and Os in HD 115444, a UMP star with $r$-process overabundances. Preliminary analysis suggests that abundances of these elements also agree with those that would have been predicted from the contents of the neutron capture elements near $Z=60$.

\section{THE $p$ PROCESS}

\section{A. The $p$ process}

One process for which $\mathrm{B}^{2} \mathrm{FH}$ anticipated much of the modern description is the $p$ process. It creates the proton-rich nuclides heavier than iron that are blocked from formation by either the $r$ or $s$ processes by stable nuclides. This definition immediately suggests the major task of any $p$-process description: how to find ways other than $\beta$ decay to process the usually more abundant $r$ and $s$-process nuclides into the usually less abundant $p$ nuclides. $\mathrm{B}^{2} \mathrm{FH}$ described two possible mechanisms by which $p$ nuclides could be formed: proton radiative captures in a hot $\left(\mathrm{T}_{9} \sim 2-3\right)$ proton-rich environment, or photon-induced $n, p$, and $\alpha$-particle removal reactions, also in a hot environment. $\mathrm{B}^{2} \mathrm{FH}$ also noted some of the modern $p$-process problem nuclei. Finally, they anticipated Type I and Type II supernovae as possible sites for the $p$ process, both of which remain fashionable today, although for rather different reasons than those suggested by $\mathrm{B}^{2} \mathrm{FH}$.

Following their qualitative description of the $p$ process, $\mathrm{B}^{2} \mathrm{FH}$ developed some numerical estimates for nuclei in the $A \sim 100$ region that suggested that $(p, \gamma)$ reactions dominated over photonuclear processes for $p$-nuclide synthesis in this mass region. They also noted that the $p$ process could proceed by successive proton capture until equilibrium between $(p, \gamma)$ and $(\gamma, p)+(\gamma, n)$ reactions was achieved, which they estimated, from assuming a generalized $\beta$ decay life-time formula, to lie 2-3 nucleons to the proton-rich side of stability. Finally they noted that proton capture rates did not appear to be a limitation to the $p$ process; captures would proceed at a sufficiently rapid rate to drive the $p$ process. However, those $\beta$-decay rates could limit the number of protons that could be added during the $p$-process event. 
The $p$ process is now thought to take place at some level in a high temperature proton-rich environment, both by proton captures on light seed nuclides and by photonuclear processes on the heaviest abundant stable nuclides. However, development of the $\alpha$-rich freezeout model in Type II supernovae, discussed elsewhere in this review, has suggested a new site for synthesis of some $p$ nuclides. This would occur along with the $r$ process in the neutrino wind resulting from supernova collapse. And other $p$ nuclides are now thought to be synthesized by neutrino-induced nucleon removal from nearby nuclides in the " $\nu$ process." Although the idea of concurrent $r, \nu$, and $p$ processes is relatively new, some features thereof are very appealing. They are discussed in greater detail below.

However, it would be premature to declare the $p$ process to be understood, partly because production of all the $p$ nuclides in the periodic table seems to require several processes (although this may not be surprising), and partly because there seem to be several explanations for production of some of those nuclides. A few of the $p$-process nuclides are so difficult to make by global mechanisms that their synthesis seems to necessitate very specific processes. In other cases, processes that seem inevitable in supernovae overproduce $p$ nuclides that are also thought to be produced in other sites, creating an apparent abundance excess. Also, the predictions of the heavy nuclide production via photodissociation are based on cross sections no one of which has been measured. Finally, it has been sufficiently difficult to identify a site in which the conditions that $p$-process models require that any $p$-process scenario must be greeted with caution.

The separate mechanisms that are suggested as contributing to $p$-process nucleosynthesis, as well as their strengths and weaknesses, are discussed below. Additional detail about the $p$ process can be found in the review article by Lambert (1992). In particular, he describes many of the details associated with determining the isotopic abundances essential to pinpointing the nature of the $p$ processes of nucleosynthesis. Specifically, studies of meteoritic samples have provided most of these details, since astronomical observation of isotopes in even the lightest $p$-process nuclides is usually not possible.

The generally accepted list of $p$ nuclides is given in Table VI. Their most general characteristic is their rarity, with a few notable exceptions, with respect to the other isotopes of each element. Note that some of them can also be made by $r$ or $s$ processes, but those processes are not thought to contribute a large fraction of any particular $p$ nuclide's total abundance.

\section{B. Early $p$-process models}

The basic features of $p$-process nucleosynthesis, and therefore some framework for the conditions required to synthesize the $p$ nuclides, were first set forth by Ito (1961). He assumed a very high-temperature hydrogenrich environment, $T_{9} \sim 2.5-3$, and $(p, \gamma)$ and $(\gamma, n)$ reac- tions to produce significant abundances of all the $p$ nuclides. Truran and Cameron (1972) studied the properties of a potential site for $p$-process nucleoysnthesis, a shock heated hydrogen-rich environment, and found that the lighter $p$ nuclides could be produced by proton capture reactions, while the photodissociative reactions were required for synthesis of the heavier nuclides, in basic agreement with $\mathrm{B}^{2} \mathrm{FH}$. They concluded that temperatures of around $\mathrm{T}_{9} \sim 2.5$ or more were required to synthesize the $p$ nuclides throughout the periodic table.

Audouze and Truran (1975) included $(p, \gamma),(n, \gamma)$, $(\alpha, \gamma),(p, n),(\alpha, p)$, and $(\alpha, n)$ reactions and their inverses in their network to study the successes and failures of the early $p$-process description. They assumed a density of $10^{4} \mathrm{~g} \mathrm{~cm}^{-3}$ and temperatures of $\mathrm{T}_{9} \sim 2$ that decreased exponentially with an $e$-folding time of either $0.446 \mathrm{~s}$, the adiabatic expansion time, or $1 \mathrm{~s}$. Their results identified some deficiencies, e.g., underproduction of the mass 92-98 $p$ nuclides. They also noted that the site for the requisite temperature and density might not exist. Arnould (1976) considered hydrostatic burning conditions that would produce simultaneous $r$ and $p$ processes, but found that very long times, greater than $10^{4} \mathrm{~s}$, were required for appreciable $p$-nuclide production. These concerns were amplified by Woosley and Howard (1978), who noted that the energy required to heat an appropriately thick hydrogen-rich shell to $T_{9} \sim 2$ for $10 \mathrm{~s}$ exceeds the entire mechanical energy output of the supernova. Woosley and Howard also found that the seed nuclei in the Audouze-Truran model would have to be enhanced in abundance by a factor of 100 for supernovae to produce the required $p$-nuclide overproduction factors. Thus, the early $p$-process description could have been correct only if a site that produces its requisite conditions can be found, and that site is not readily identifiable with either supernovae or novae.

\section{The $\gamma$ process}

The $\gamma$ process appears to be capable of producing nearly all the heavy $(A>100) p$ nuclei. As noted above, it was first studied by Ito (1961) and advanced by Audouze and Truran (1975). The current description was formulated two decades ago by Woosley and Howard (1978) who showed that, with a few notable exceptions, the predicted ratios of the abundances of all the heavy $p$ nuclides corresponded reasonably well with those observed. One of the exceptions, ${ }^{146} \mathrm{Sm}$, was subsequently explained (Woosley and Howard, 1990) by a more careful handling of the nuclear physics details. The other two, ${ }^{180} \mathrm{Ta}$ and ${ }^{138} \mathrm{La}$, are discussed below. Both solar and $s$ process enhanced seeds were tried. The latter, of course, enhanced the $p$-nuclide abundances, but did little redistribution thereof. The Woosley-Howard work was updated in 1990 (Woosley and Howard, 1990).

The $\gamma$ process operates essentially as the $\mathrm{B}^{2} \mathrm{FH} p$ process, i.e., photons from a high-temperature $\left(\mathrm{T}_{9} \sim 2-3\right)$ bath containing previously synthesized heavy nuclides initiate successive $(\gamma, p),(\gamma, n)$, and $(\gamma, \alpha)$ reactions on 
TABLE VI. The $p$-process nuclides.

\begin{tabular}{|c|c|c|c|c|c|}
\hline Element & $Z$ & $A$ & $N_{A}^{\mathrm{a}}$ & $\left(N_{A} / \Sigma N_{A}\right) \times 100$ & Comments \\
\hline $\mathrm{Se}$ & 34 & 74 & 0.55 & 0.88 & \\
\hline $\mathrm{Kr}$ & 36 & 78 & 0.15 & 0.34 & \\
\hline $\mathrm{Sr}$ & 38 & 84 & 0.13 & 0.56 & \\
\hline $\mathrm{Nb}$ & 41 & 92 & 0.0 & 0.0 & $\beta$ decays to ${ }^{92} \mathrm{Mo} ;\left(T_{1 / 2}\right)^{\mathrm{b}}=3.5 \times 10^{7} \mathrm{y}$ \\
\hline \multirow[t]{2}{*}{ Mo } & 42 & 92 & 0.38 & 14.84 & \\
\hline & & 94 & 0.24 & 9.25 & Also produced by $s$ process \\
\hline \multirow[t]{2}{*}{$\mathrm{Ru}$} & 44 & 96 & 0.10 & 5.52 & \\
\hline & & 98 & 0.035 & 1.88 & \\
\hline $\mathrm{Pd}$ & 46 & 102 & 0.014 & 1.02 & \\
\hline \multirow[t]{2}{*}{$\mathrm{Cd}$} & 48 & 106 & 0.020 & 1.25 & \\
\hline & & 108 & 0.014 & 0.89 & \\
\hline In & 49 & 113 & $7.9 \times 10^{-3}$ & 4.3 & Also produced by $r$ and $s$ processes \\
\hline \multirow[t]{2}{*}{ Sn } & 50 & 112 & 0.037 & 0.97 & \\
\hline & & 114 & 0.025 & 0.66 & Also produced by $s$ process \\
\hline $\mathrm{Te}$ & 52 & 120 & $4.3 \times 10^{-3}$ & 0.09 & \\
\hline \multirow[t]{2}{*}{$\mathrm{Xe}$} & 54 & 124 & $5.7 \times 10^{-3}$ & 0.12 & \\
\hline & & 126 & $5.1 \times 10^{-3}$ & 0.11 & \\
\hline \multirow[t]{2}{*}{$\mathrm{Ba}$} & 56 & 130 & $4.8 \times 10^{-3}$ & 0.11 & \\
\hline & & 132 & $4.5 \times 10^{-3}$ & 0.10 & \\
\hline $\mathrm{La}$ & 57 & 138 & $4.1 \times 10^{-3}$ & 0.09 & $\beta$ decays to ${ }^{138} \mathrm{Ce} ;\left(T_{1 / 2}\right)^{\mathrm{b}}=1.5 \times 10^{11} \mathrm{y}$ \\
\hline \multirow[t]{2}{*}{$\mathrm{Ce}$} & 58 & 136 & $2.2 \times 10^{-3}$ & 0.19 & \\
\hline & & 138 & $2.8 \times 10^{-3}$ & 0.25 & \\
\hline \multirow[t]{2}{*}{$\mathrm{Sm}$} & 62 & 144 & $8.0 \times 10^{-3}$ & 3.10 & \\
\hline & & 146 & 0.0 & 0.0 & $\alpha$ decays to ${ }^{142} \mathrm{Nd} ;\left(T_{1 / 2}\right)^{\mathrm{b}}=1.03 \times 10^{8} \mathrm{y}$ \\
\hline \multirow[t]{2}{*}{ Dy } & 66 & 156 & $2.2 \times 10^{-4}$ & 0.06 & \\
\hline & & 158 & $3.8 \times 10^{-4}$ & 0.10 & \\
\hline $\mathrm{Er}$ & 68 & 162 & $3.5 \times 10^{-4}$ & 0.14 & \\
\hline $\mathrm{Yb}$ & 70 & 168 & $3.2 \times 10^{-4}$ & 0.13 & \\
\hline Hf & 72 & 174 & $2.5 \times 10^{-4}$ & 0.16 & \\
\hline $\mathrm{Ta}$ & 73 & 180 & $2.5 \times 10^{-6}$ & 0.01 & $\begin{array}{l}\text { Actually }{ }^{180} \mathrm{Ta}^{m} ;\left(T_{1 / 2}\right)^{\mathrm{b}}=1.2 \times 10^{15} \mathrm{y} \\
\beta^{-} \text {decays to }{ }^{180} \mathrm{~W} \text { or } e^{-} \text {captures to }{ }^{180} \mathrm{Hf} \\
\text { Also produced by } s \text { process }\end{array}$ \\
\hline $\mathrm{W}$ & 74 & 180 & $1.7 \times 10^{-4}$ & 0.13 & Also possibly produced by $s$ process \\
\hline Os & 76 & 184 & $1.2 \times 10^{-4}$ & 0.02 & \\
\hline $\mathrm{Pt}$ & 78 & 190 & $1.7 \times 10^{-4}$ & 0.01 & \\
\hline $\mathrm{Hg}$ & 80 & 196 & $5.2 \times 10^{-4}$ & 0.15 & \\
\hline
\end{tabular}

those nuclides to synthesize the heavy $p$ nuclides. Typical processing times must be less than a second, as longer times would, at these high temperatures, destroy all the heavy nuclides by photonuclear processes. Blocking of the $\beta^{-}$decays from the abundant nuclides on the neutron-rich side of stability to the $p$ nuclides, the standard inhibition to $p$-nuclide production, is circumvented by the photon-induced reactions that populate the $p$ nuclides directly. For example, any sequence of photonuclear reactions in which 14 neutrons and 4 protons are removed from ${ }^{208} \mathrm{~Pb}$ would produce the $p$ nuclide ${ }^{190} \mathrm{Pt}$. Initially the $(\gamma, n)$ reactions would dominate, but $(\gamma, p)$ and $(\gamma, \alpha)$ reactions would become more probable as the photodisintegrations proceeded. In some cases proton-rich unstable nuclides are formed which $\beta^{+}$decay back to the stable $p$ nuclides after the $\gamma$-process conditions have subsided. Of course, the $Q$ values and, for charged particles, Coulomb barriers will determine the probability of each reaction from any intermediate nucleus. In addition to photonuclear production, the light $p$ nuclides might also be formed by rapid proton captures on lighter (and more abundant) seed nuclides, in which the seed nuclei are driven to the proton drip line. They decay back to stability after the high temperature environment has cooled.

A major issue for the $\gamma$ process appears to be whether or not a site could be found for it that would yield appropriate overproduction factors for the $p$ nuclides. Rayet et al. (1990) extended the original WoosleyHoward work to an oxygen burning shell of a supernova. Their calculations involved $(n, \gamma),(p, \gamma),(\alpha, \gamma)$, $(n, p),(n, \alpha)$, and $(\mathrm{p}, \alpha)$ reactions and their inverses, as 
well as reactions between several heavy nuclei. The peak temperatures ranged from $\mathrm{T}_{9}=2.2-3.2$, and fell off exponentially with an e-folding time of either $0.446 \mathrm{~s}$ or $1 \mathrm{~s}$, and densities were $10^{6} \mathrm{~g} \mathrm{~cm}^{-1}$. Despite the differences between the studies of Rayet et al. (1990) and of Woosley and Howard (1978), their results did not differ from each other in most cases by more than a factor of 2 .

Prantzos et al. (1990) extended the study of Rayet et al. (1990) by using their reaction network in a model of the thermodynamic conditions of a Type II supernova. The model used had been found (Nomoto and Hashimoto, 1988) to give a good representation of SN 1987A. The temperatures involved ranged from $\mathrm{T}_{9}$ of 2 to 3.2 which was found to be the range relevant to the $p$ process, as very little processing occurred at lower temperatures, and the seeds were destroyed at higher temperatures. It was assumed that the seed nuclei were those resulting from the $s$ process. Finally Rayet et al. (1995) considered $p$-nuclide production from Type II supernovae spanning a mass range from 13 to $25 M_{\odot}$. The results of these three studies were significant $p$-nuclide overproduction factors, but a wide variation in their relative abundances. Most notably, the Mo and $\mathrm{Ru} p$ nuclides were badly underproduced, as were a number of others. However, it should be noted that the overproduction factors of the $p$ nuclides lighter than Mo were roughly the same as those for the more massive nuclides excepting the usual $p$-nuclide problems. It is noteworthy that the model of Prantzos et al. (1990) produced roughly the correct overproduction factor of ${ }^{180} \mathrm{Ta}$, a nuclide that is a problem for all other $\gamma$-process models.

A recent extension of the Woosley-Howard work by Howard et al. (1991) has identified a plausible site for the $\gamma$ process. They find the requisite thermodynamic and seed conditions to occur when a carbon-oxygen white dwarf explodes, either as Type Ia or a subclass of a Type II supernova. The resulting high temperatures, $\mathrm{T}_{9} \sim 2-3$, produce the high-energy photons necessary to photodissociate the heavy nuclear seeds to yield the heavy $p$ nuclides, and the carbon-burning reactions of the white dwarf produce enough protons to fuel the $(p, \gamma)$ reactions necessary to synthesize the lighter $p$ nuclides. Virtually all of the $p$ nuclei heavier than ${ }^{92} \mathrm{Mo}$ have overproduction factors within a factor of 9 of each other (excepting ${ }^{180} \mathrm{Ta},{ }^{146} \mathrm{Sm}$, and ${ }^{138} \mathrm{La}$ ), and absolute overproduction factors of order $10^{4}$, a number large enough to suggest that the site should be taken seriously. The overproduction factors (ratios of averaged stellar to solar system mass fractions) of order of the $p$ nuclides from this model are shown in Fig. 23.

The exploding carbon-oxygen white dwarf model does have some deficiencies, even aside from ${ }^{180} \mathrm{Ta}$ and ${ }^{138} \mathrm{La}$. Specifically, ${ }^{152} \mathrm{Gd}$ is badly underproduced, and the light $p$ nuclides are overproduced relative to their more massive counterparts. In addition, the ${ }^{74} \mathrm{Se},{ }^{78} \mathrm{Kr}$, and ${ }^{84} \mathrm{Sr}$ overproduction factors fall as much as a factor of 2 above those of the heavier nuclides. While this alone might not constitute a fatal flaw, other processes also produce those lighter $p$ nuclides. The resulting total overproduction would thus present a serious conflict

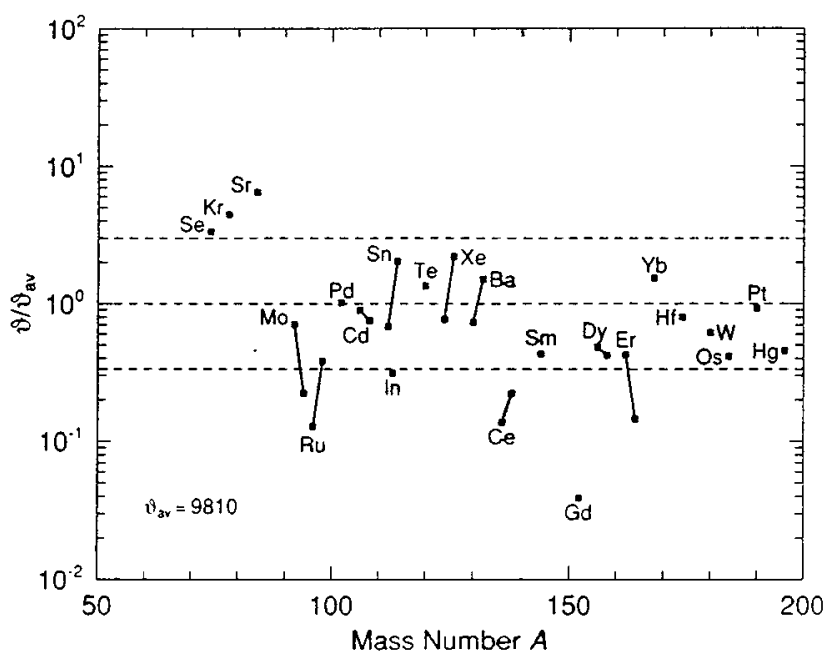

FIG. 23. Overproduction factors for $p$ nuclides, relative to the average overproduction factor, as a function of atomic mass. The values shown are an average of equally weighted contributions from temperatures in the range $\mathrm{T}_{9}=2.4$ to 3.2 in steps of 0.1. From Howard et al. (1991).

with the observed abundances. Finally, as usual, the Mo and $\mathrm{Ru} p$ nuclides are underproduced.

Essential inputs to any $\gamma$-process description are the properties of the very proton-rich lighter nuclides that are produced primarily by rapid proton captures. In particular, the masses are crucial, as the high temperature environment that characterizes the $\gamma$ process will certainly produce $(\gamma, p)$ and $(\gamma, \alpha)$ reactions to compete with the $(p, \gamma)$ reactions, and the probabilities of the photonuclear processes depend critically on the reaction $Q$ values. Those nuclides with long half-lives will tend to inhibit the flow of the $\gamma$ process and will build up in abundance. Finally, the decay modes may be important as, e.g., $\beta^{+}$decay and $\beta^{+}-p$ decay will produce different final nuclides from the $\gamma$ process. Thus significant effort has gone into observing these properties of the very proton-rich nuclides (Boyd, 1994; Winger et al., 1993; Blank et al., 1995).

\section{The $r p$ process}

Some nucleosynthesis of the lighter $p$ nuclides is provided by the $r p$ process. The modern formulation of the $r p$ process, proposed by Wallace and Woosley (1981) and described also in Sec. IV.H, describes the explosive nucleosynthesis that would occur in a fairly hightemperature hydrogen-rich environment. Wallace and Woosley considered temperatures as high as $\mathrm{T}_{9} \sim 2$, and found that even at temperatures appreciably less than a $\mathrm{T}_{9}$ of 1 they could produce appreciable abundances of ${ }^{74} \mathrm{Se}$ and ${ }^{78} \mathrm{Kr}$. Possible sites for such environments could result from accretion from the periphery of a red giant onto a companion white dwarf or neutron star. The seed nuclei contained in the $r p$ process environment are driven to the proton-drip line, from which they decay after the high temperatures have subsided. Note 
that, at high temperatures, the $r p$ and $\gamma$ processes are qualitatively similar; high-energy photons photodissociate the heavy nuclei and proton radiative captures boost the masses of the lighter nuclei.

Beyond iron, the $r p$ process begins to populate some of the nuclides that are the progenitors of the $p$ nuclides. The details of the burning were worked out by van Wormer et al. (1994), who assumed (constant) temperatures ranging from $\mathrm{T}_{9}$ of $0.15-1.5$, densities around $10^{4} \mathrm{~g}$ $\mathrm{cm}^{-3}$, and processing times of up to $1000 \mathrm{~s}$ for the lowest temperatures. At the upper end of their temperature range significant abundances of ${ }^{74} \mathrm{Se}$ and ${ }^{78} \mathrm{Kr}$ could be produced. However, ${ }^{69} \mathrm{Br}$, which might be thought to be formed by ${ }^{68} \mathrm{Se}(p, \gamma){ }^{69} \mathrm{Br}$, was subsequently found to be proton unbound (Blank et al., 1995). Since the half-life of ${ }^{68} \mathrm{Se}$ is $35.5 \mathrm{~s}$, appreciably longer than the highest temperature conditions can persist, the $r p$ process will be slowed appreciably at mass 68 . Of course, some leakage past ${ }^{68} \mathrm{Se}$ will occur from the decays that do occur, and this could be a fairly large fraction of those that end up at ${ }^{68} \mathrm{Se}$ if the high-temperature conditions last a significant fraction of the ${ }^{68} \mathrm{Se}$ half-life.

The yields were reexamined by Herndl et al. (1995) with reaction rates determined from the nuclear structure results of shell model calculations. The possibility that two-proton captures could influence the results were also studied (Schatz et al., 1997). It was found that the usual $r p$-process termination points could be circumvented, but only at rather extreme conditions $\left(T_{9}=1=1.8\right.$, density $\left.=10^{6} \mathrm{~g} \mathrm{~cm}^{-3}\right)$. In such environments, the first capture might also circumvent the termination points (Schatz et al., 1997) in particularly hot dense environments, as the first might result in a sufficiently long-lived state that a second can occur before the initial proton is reemitted. Finally, Thielemann et al. (1994) studied the general features of the $r p$-process path, most notably, that it tends to involve cycles superimposed on its general flow. Indeed the general features of the $r p$ process were found to be considerably more complex than those of, e.g., the $r$ process, due to the interplay of proton captures, $\beta$ decays, possible photodisintegrations, and $\alpha$ particle induced reactions throughout the $r p$-process path.

Radioactive beam studies have made the present description of the $r p$-process possible. It had been thought that ${ }^{64} \mathrm{Ge}$ would be the end of the $r p$ process, as mass formulae suggested that ${ }^{65} \mathrm{As}$, the nucleus formed by ${ }^{64} \mathrm{Ge}(p, \gamma){ }^{65} \mathrm{As}$, would be unstable to proton decay. This, however, was found (Winger et al., 1993) not to be the case, allowing the $r p$ process to proceed up to ${ }^{68} \mathrm{Se}$.

The ${ }^{68} \mathrm{Se}$ roadblock would also be circumvented if the $r p$ process occurred in a Thorne-Żytkow object (Biehle, 1991), a hypothetical star in its red giant phase that has a neutron star at its center. Such a composite star would be sufficiently convective that material might undergo multiple processing episodes at the high temperature region near the surface of the neutron star. Thus the proton rich nuclides synthesized in each processing interval would undergo $\beta^{+}$decay to provide seeds for the next processing sequence. Such objects might be expected to make copious amounts of the light $p$-process nuclides (Cannon, 1993), although, as shown by van Wormer et al. (1994), $T_{9}$ must be less than about 2 or $(\gamma, \alpha)$ reactions will terminate the nucleosynthesis at about mass 56. In addition, recent studies (Chevalier, 1996; Fryer et al., 1996) have cast some doubt on the stability of a dense gas just above the surface of a neutron star. Most troublesome, though, is the absence of a definitive discovery of a Thorne-Zytkow object.

\section{E. The $\nu$ process}

In 1990, the $\nu$ process was proposed by Woosley et al. (1990), after having first been suggested by Domogatskii et al. (1977), and was shown to be capable of producing some of the lowest abundance $p$ nuclides. The process is thought to occur in the neutrino wind generated by supernova collapse. The nuclides synthesized clearly depend on the shell in which the $\nu$ process occurs. For example, ${ }^{11} \mathrm{~B}$ would be expected to be made in a shell in which the dominant constituent was ${ }^{12} \mathrm{C}$, and ${ }^{19} \mathrm{~F}$ would be made in a shell with ${ }^{20} \mathrm{Ne}$, both by processes in which a neutrino would excite the target nucleus via the neutral-current interaction (which therefore allows interactions with all neutrino flavors) to an excited state from which the nucleus could emit either a proton or a neutron.

However, the $\nu$ process could make two of the rarest stable nuclides in the periodic table: ${ }^{138} \mathrm{La}$ and ${ }^{180} \mathrm{Ta}$. ${ }^{180} \mathrm{Ta}$ would be made by the ${ }^{181} \mathrm{Ta}\left(\nu, \nu^{\prime} n\right)^{180} \mathrm{Ta}$ (neutral current) reaction, which appears to produce an abundance consistent with that observed. Similarly, the ${ }^{139} \mathrm{La}\left(\nu, \nu^{\prime} n\right){ }^{138} \mathrm{La}$ reaction produces ${ }^{138} \mathrm{La}$. However, it appears that a comparable amount of ${ }^{138} \mathrm{La}$ would also be produced by the ${ }^{138} \mathrm{Ba}\left(\nu, e^{-}\right){ }^{138} \mathrm{La}$ (charged current) reaction; these two reactions appear capable of synthesizing roughly the observed ${ }^{138} \mathrm{La}$ abundance.

Note that the requirement of heavy seeds for production of ${ }^{138} \mathrm{La}$ and ${ }^{180} \mathrm{Ta}$ is not in conflict with the photodissociation to $\alpha$ particles and neutrons that occurs during $\alpha$-rich freeze out, discussed in Sec. XII and briefly below. The $\nu$ process occurs outside the bubble in which the $\alpha$-rich freeze out occurs, so it can operate on its requisite previously synthesized nuclei. Indeed, the $\nu$ process seems to provide a natural mechanism for synthesis of ${ }^{138} \mathrm{La}$ and ${ }^{180} \mathrm{Ta}$, whose synthesis has evaded description for several decades.

While the $\nu$ process appears very promising for synthesizing some of the more difficult to explain $p$-process nuclides, its results are somewhat in question due to uncertainties about the neutrino spectrum resulting from a Type II supernova (Myra and Burrows, 1990) and about the neutrino cross sections. Thus its full acceptance requires that the details of its nucleosynthesis be worked into a realistic supernova model.

\section{F. Recent developments}

In the past several years the $p$ process, along with the $r$ and $\nu$ processes, has undergone a revolution that might 
solve some of the most troublesome problems of nucleosynthesis. The process that has emerged is $\alpha$-rich freeze out in neutrino-driven winds occuring after core bounce in a supernova (Woosley and Hoffman, 1992); this shows promise of providing a site for both the $r$ and $\nu$ processes in addition to a site for synthesis of some of the most challenging $p$ nuclides. This process occurs about $1 \mathrm{~s}$ after a supernova collapse undergoes core bounce in the high-entropy high-temperature (initially $\left.\mathrm{T}_{9} \sim 10\right)$ bubble near the core.

In this process, the photons in the high temperature environment dissociate all the preexisting nuclei down to essentially $\alpha$ particles and neutrons. A critical parameter in characterizing the environment is the electron fraction $Y_{e}=p /(n+p)$, where $n$ and $p$ are the neutron and proton densities. As the environment cools, nuclear statistical equilibrium dictates the abundances created until the nuclear reactions initiated with charged particles freeze out. Then the remaining neutrons synthesize all the $r$-process nuclides, provided that $Y_{e}$ is appropriately chosen. This environment has also been shown to create the light $p$ nuclides through ${ }^{92} \mathrm{Mo}$ (Hoffman et al., 1996), both in the correct relative abundances and in absolute magnitude, again if $Y_{e}$ is chosen appropriately. Some of these are actually synthesized from nearby $r$-process products through charged current interactions with the electron neutrinos. For example (Fuller and Meyer, 1995), neutrino capture will dominate over antineutrino capture, so that processes such as ${ }^{92} \mathrm{Zr}\left(\nu, e^{-}\right){ }^{92} \mathrm{Nb}$ and ${ }^{92} \mathrm{Nb}\left(\nu, e^{-}\right){ }^{92}$ Mo become possible, and could make a great deal of ${ }^{92} \mathrm{Mo}$. Since such processes can proceed through stable nuclei, they do allow production of $p$ nuclides from $r$ - and $s$-process seeds. However, the extent to which such processing can occur is limited, as too many neutrino-initiated interactions would smear out and shift the well-established $r$-process abundance peaks resulting from the neutron closed shells.

The $\alpha$-rich freeze-out model does have some deficiencies that have persisted since its inception. Those associated with the $r$ process are discussed in Sec. XII. From the perspective of the $p$ process; however, although this process does make ${ }^{92}$ Mo with enough abundance to provide all that observed in nature, it is unable to produce abundances even close to those of the next several $p$ nuclides. In addition, the results of the model calculations exhibit an uncomfortable sensitivity to $Y_{e}$. Only future work will tell if these problems can be solved, and the $\alpha$-rich freeze-out model can provide the description of the nucleosynthesis of at least part of the $p$ nuclides.

\section{G. Summary}

Although the various subprocesses by which the synthesis of the $p$ nuclides is explained seem promising, some of them are not yet fully developed. Furthermore, the overview study of these processes that is required to demonstrate that together they produce the correct overproduction factors of the $p$ nuclides has certainly not been done. Most notably, the promising $\alpha$-rich freeze-out model produces appropriate abundances of the $p$ nuclides up through ${ }^{92}$ Mo. But the exploding white dwarf model also produces those same nuclides, excepting ${ }^{92} \mathrm{Mo}$, at such abundances, as to possibly create an excess of those nuclides. And no model has yet shown how to produce ${ }^{94} \mathrm{Mo},{ }^{96} \mathrm{Ru}$, and ${ }^{98} \mathrm{Ru}$ in adequate quantities. Thus, although the present $p$-process descriptions have advanced greatly beyond that of $\mathrm{B}^{2} \mathrm{FH}$, serious questions remain as to the viability of our present descriptions of that process.

\section{THE e PROCESS AND THE IRON-GROUP NUCLEI}

\section{A. Energetics and equilibria}

In even a cursory glance at the abundance distribution of nuclides in the solar system, the iron peak leaps out at the eye. It stretches from nuclear mass number $A \approx 40$ to $A \approx 60$, comprising isotopes from $\mathrm{Ca}$ to $\mathrm{Zn}$. The peak is at ${ }^{56} \mathrm{Fe}$, which is the 10 th most abundant species in the solar system. ${ }^{56} \mathrm{Fe}$ makes up $\sim 0.1 \%$ of the solar system's mass. This is even more impressive when one realizes that ${ }^{56} \mathrm{Fe}$ is $\sim 6 \%$ of the solar system's mass in species other than $\mathrm{H}$ and $\mathrm{He}$ and $\sim 20 \%$ of the mass in everything other than $\mathrm{H}, \mathrm{He}, \mathrm{C}, \mathrm{N}$, and $\mathrm{O}$ ! The other irongroup isotopes are less abundant than ${ }^{56} \mathrm{Fe}$, but their contribution to the mass of the solar system is still significant: $\sim 5 \%$ of the contribution of ${ }^{56} \mathrm{Fe}$. Clearly something special about ${ }^{56} \mathrm{Fe}$ and its iron-group neighbors makes them so abundant.

That something special is their strong nuclear binding energy: the iron-group nuclides are the most tightly bound isotopes in nature. The binding energy $B(Z, N)$ of a nucleus with $Z$ protons and $N$ neutrons is

$$
B(Z, N)=Z m_{p} c^{2}+N m_{n} c^{2}-M(Z, N) c^{2},
$$

where $m_{p}$ and $m_{n}$ are the masses of the proton and neutron, respectively, and $M(Z, N)$ is the mass of the nucleus $(Z, N)$. Figure 24 illustrates the strong binding of the iron-group isotopes. Among isotopes with equal numbers of neutrons and protons, the iron-group nuclide ${ }^{56} \mathrm{Ni}$ has the greatest binding energy per nucleon. As defined in Eq. (28), the larger the binding energy, the smaller the nuclear mass relative to the total mass of the constituent nucleons, and the more tightly bound the nucleus. The binding even increases for somewhat more neutron-rich isotopes. For isotopes with $0.86 \leqslant \mathrm{Z} /$ $\mathrm{N} \leqslant 0.88$, Fig. 24 shows that the dominant iron-group nuclide ${ }^{56} \mathrm{Fe}$ is the most tightly bound isotope. And among all nuclei, the iron-group isotope ${ }^{62} \mathrm{Ni}$ has the largest binding energy per nucleon (8.794 MeV) according to the mass compilation of Tuli (1995).

The strong binding energy per nucleon of iron-group nuclei means that these isotopes will tend to dominate the nuclear abundances if the system reaches nuclear equilibrium. Hoyle (1946) recognized this fact, and $\mathrm{B}^{2} \mathrm{FH}$ elaborated on it. In the intervening years, the progress in understanding the synthesis of iron-group nuclei has been in a broadening of the picture of possible statistical equilibria and how nature realizes these 

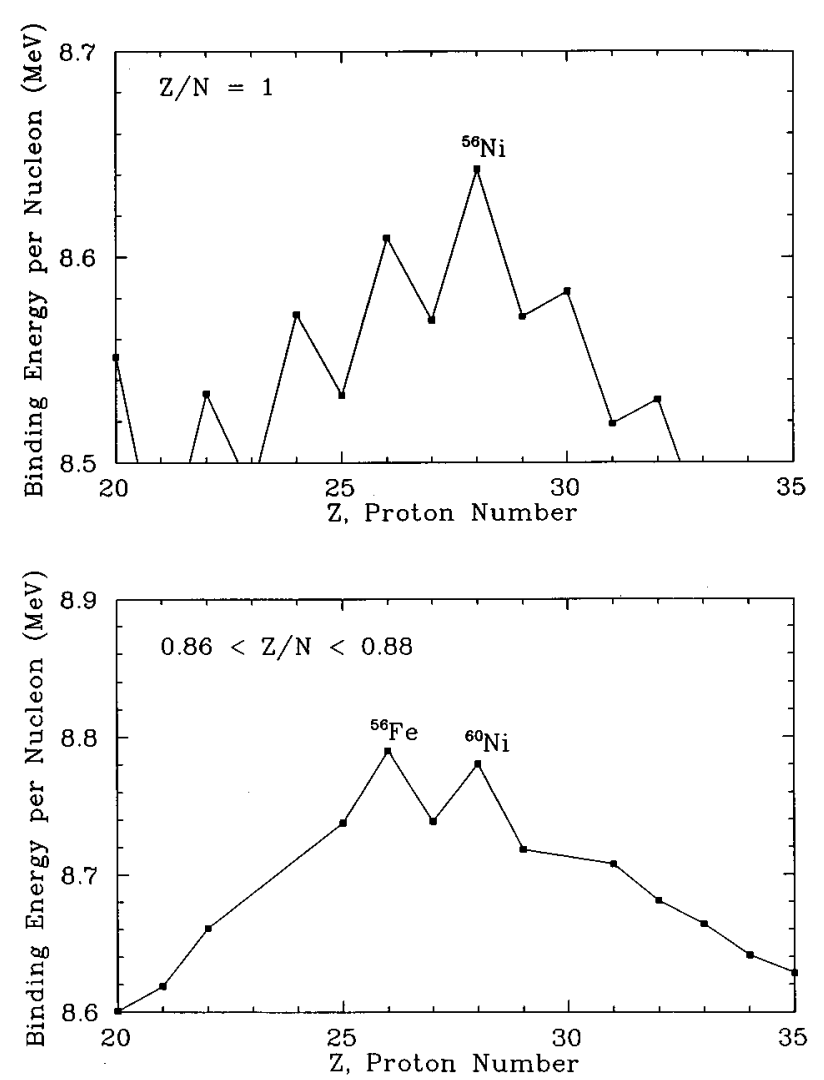

FIG. 24. The binding energy per nucleon in $\mathrm{MeV}$ for nuclides with $Z=N$ (top panel) and with $0.86 \leqslant Z / N \leqslant 0.88$ (bottom panel). ${ }^{56} \mathrm{Ni}$ has the strongest binding energy per nucleon for $Z=N$ nuclei while ${ }^{56} \mathrm{Fe}$ has the strongest binding for nuclei with $Z / N \approx 0.87$. ${ }^{60} \mathrm{Ni}$ is also tightly bound, but not as much as ${ }^{56} \mathrm{Fe}$. No isotope of vanadium $(Z=23)$ or chromium $(Z=24)$ has $0.86 \leqslant Z / N \leqslant 0.88$. ${ }^{56} \mathrm{Fe}$ could be made as itself in equilibrium if the ratio of neutrons to protons in the nucleosynthetic environment were around 0.87 . This is $\mathrm{B}^{2} \mathrm{FH}$ 's $e$ process. In fact, nature seems to have chosen to assemble most of the solar system's iron-group nuclei in matter that had equal numbers of neutrons and protons. In this case, ${ }^{56} \mathrm{Ni}$ was made and later decayed to ${ }^{56} \mathrm{Fe}$. The masses needed for this figure are from Tuli (1995).

equilibria. Summarizing this progress is the dominant theme of this review. Before proceeding to this, however, a few further comments on the nuclear physics of the iron-group nuclei are in order.

Figure 25 shows the binding energy per nucleon for the $A=28,32,36,40,44$, and 56 isobars (isobars are nuclides with the same mass number $A$ ). What is clear is that for $A>32$, the $Z=N$ nuclide is not the most tightly bound isobar. This is understandable in terms of the liquid-drop model of the nucleus (von Weizsäcker, 1935; for a modern descendant of this model, see Möller et al., 1995). In this model, the nuclear mass is the sum of (1) the masses of the constituent neutrons and protons, (2) a volume term, (3) a surface term, (4) a symmetry term, and (5) a Coulomb term. Refinements account for the single-particle effects of nuclear pairing and the nuclear shells. It is decisive that the symmetry term falls off with $A$ as $[(A / 2)-Z]^{2} / A$ while the Coulomb term grows with $Z$ as $Z^{2} / A^{1 / 3}$. For nuclei with $A \lesssim 32$, the symmetry term is sufficiently important relative to the Coulomb energy that $Z=N$ nuclides can be the most bound isobar. Nuclear pairing favors even- $Z-$-even- $N$ nuclei over odd- $Z$-odd- $N$ nuclei, which explains why ${ }^{30} \mathrm{P}$, a $Z=N$ nuclide, is less bound than ${ }^{30} \mathrm{Si}$. By contrast, the Coulomb term begins to dominate for nuclei with $Z>16$, and the $Z=N$ nuclide is no longer the most bound isobar. Nevertheless, for $A=36$ and $A=40$ the $Z=N$ nuclide is still stable. In the case of $A=36$, although ${ }^{36} \mathrm{Cl}$ is more tightly bound than ${ }^{36} \mathrm{Ar}$, the total mass of the nucleons in ${ }^{36} \mathrm{Cl}$ is greater than that in ${ }^{36} \mathrm{Ar}$. As a consequence, the mass of ${ }^{36} \mathrm{Ar}$ is in fact less than that of ${ }^{36} \mathrm{Cl}$. This means that the decay ${ }^{36} \mathrm{Ar} \rightarrow{ }^{36} \mathrm{Cl}$ does not occur. For $A=40,{ }^{40} \mathrm{Ca}$ is more bound than ${ }^{40} \mathrm{~K}$. Above $A=40$, however, the Coulomb energy is so strong that it dominates the single-particle effects that allowed lower mass $Z=N$ nuclides to be stable. The consequence is that there is no stable $Z=N$ nuclide having $A>40$, and the iron-group elements are the first not to have stable $Z=N$ isotopes. Because nature has chosen to synthesize the bulk of the iron-group isotopes in matter with equal numbers of neutrons and protons, these isotopes generally had radioactive progenitors. This has enormous implications for astronomy, but it was not known to $\mathrm{B}^{2} \mathrm{FH}$ !

\section{B. Statistical equilibrium}

The idea of statistical equilibrium has played a special role in the history of stellar nucleosynthesis theory. Hoyle recognized in 1946 that the dramatic peak in the abundances of the iron-group nuclei called for synthesis under conditions of temperature and density such that statistical equilibrium was attained between nuclei and free neutrons and protons, and he showed that evolved stars reached appropriate thermal conditions (Hoyle, 1946). This achievement launched the paradigm of element formation in stars and led eleven years later to the still-celebrated review by $\mathrm{B}^{2} \mathrm{FH}$. The name that $\mathrm{B}^{2} \mathrm{FH}$ bestowed on this mode of element synthesis was the " $e$ process" (for "equilibrium"). $\mathrm{B}^{2} \mathrm{FH}$ had a rather specific definition in mind for the $e$ process, one using the nuclear properties of iron; but modern usage of the term often is looser than the original in simply focusing on statistical equilibrium. This review will distinguish between $\mathrm{B}^{2} \mathrm{FH}$ 's $e$ process, which ultimately was not the correct explanation for the origin of the bulk of the solar system's iron isotopic abundances, and the more general notion of nuclear statistical equilibrium (NSE). That distinction facilitates better understanding of modern ideas of iron-group elements synthesis and of the historical development of those ideas.

The essential statistical notions have not changed from those contained in Hoyle (1946) and $\mathrm{B}^{2} \mathrm{FH}$. The years since 1957 have, however, seen a broadening of these ideas. It is now becoming clear that a hierarchy of possible statistical equilibria exist and that differing natural systems can achieve these equilibria to high accuracy. This perspective may not be familiar to most 

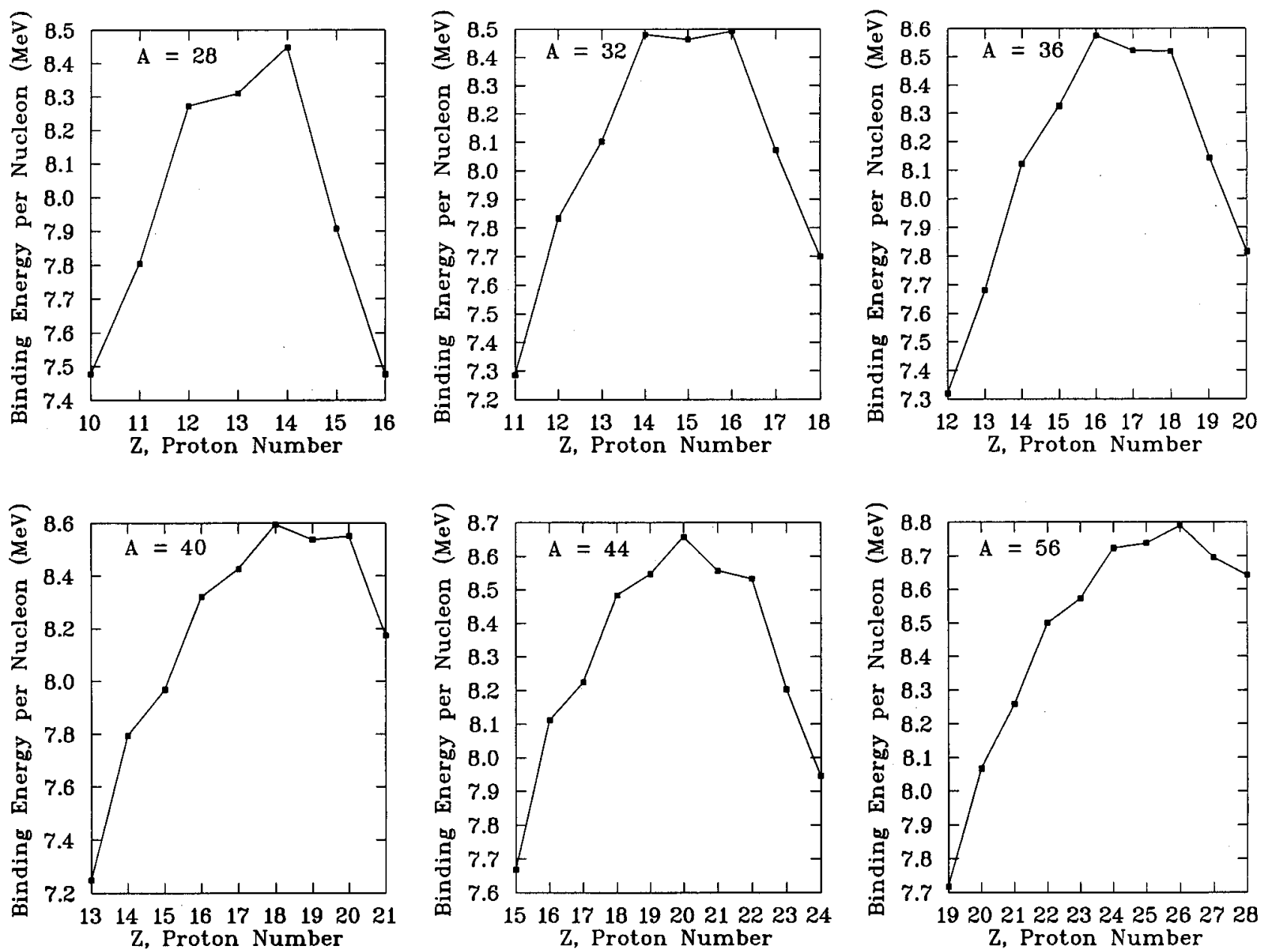

FIG. 25. The binding energy per nucleon of the isobars with $A=24,32,36,40,44$, and 56 . Only for $A \leqslant 32$ is the $Z=N$ nuclide the most bound isobar. For $A>32$ the $Z=N$ nuclide is not the most bound isobar. This is due to the fact that the Coulomb energy in these nuclei is sufficiently strong that it favors fewer protons and more neutrons. Nevertheless, the $Z=N$ isobars of $A=36$ and $A=40$ are still stable. For nuclei with $A>40$, however, the Coulomb energy is so strong that the $Z=N$ nuclide is no longer stable. Nature has chosen to assemble most of the iron-group nuclei present in the solar system in matter with equal numbers of neutrons and protons. As a consequence of that choice, most of these nuclei were originally synthesized as radioactive $Z=N$ progenitors. The masses needed for this figure are from Tuli (1995).

readers of this review, and it may require some effort to develop. Nevertheless, the insight garnered is well worth the intellectual investment.

The entropy of a system provides the most natural framework for understanding statistical equilibrium. The entropy $S$ is properly a function of the energy $E$, the volume $V$, and the numbers $\left\{N_{i}\right\}$ of each species $i$ in the system. $S$ is defined as

$$
S\left(E, V,\left\{N_{i}\right\}\right)=k \ln \Gamma,
$$

where $k$ is Boltzmann's constant and $\Gamma$ is the number of available allowed macroscopic states of the system. A macroscopic state is a particular arrangement of the various species $i$, and an allowed macroscopic state is one that has energy $E$. For nucleosynthetic systems, for example, a macroscopic state would be a particular set of abundances of nuclear species, but arranged in such a way that the total energy is $E$.

The value of the concept of the entropy is that it gives a clear notion of the evolution of a system. If the system is thermally isolated, and the volume is left constant, the natural evolution is towards maximum entropy. If any macroscopic states with energy $E$ were not available to the system at time $t_{1}$, the system evolves in such a way as to make those states available at a later time $t_{2}$. The system will continue to evolve until all allowed macroscopic states are available. Once this condition is attained, the entropy is a maximum, and there is no further evolution. The system has reached complete statistical equilibrium.

Systems rarely reach complete statistical equilibrium. The problem is that some key interaction time scale or time scales may exceed the duration of the natural event. This means that a subset of allowed macroscopic states will be closed off to the system. Nevertheless, if all other interaction time scales are sufficiently short, all allowed macroscopic states excluding those in the closed off subset will be available. That system maximizes the entropy as best it can, but its search is "constrained" to the available subset of states. This leads to a hierarchy of 
possible statistical equilibria in which systems have maximum entropy subject to different numbers of constraints.

Stellar nucleosynthesis systems present an example of such a constrained system. Conditions in stars are rarely, if ever, sufficiently extreme to allow nucleons to convert into other types of subatomic particles such as hyperons or free quarks. The time scale for the nucleons to come into equilibrium with these other particles is too long. This means that some macroscopic states with energy $E$, but with hyperons present in equilibrium with the nucleons, are not available to the system. The system is thus constrained to a smaller number of macroscopic states than the number in the full ensemble. This constraint may be incorporated by writing

$$
\sum_{i} A_{i} Y_{i}=1
$$

where $A_{i}$ is the mass number of species $i, Y_{i}$ the abundance per nucleon of species $i$, and the sum runs only over nuclear species.

Statistical equilibrium is an entropy extremum, so it is found by considering changes in the entropy. In the thermally isolated system, infinitesimal, quasistatic changes in the entropy per nucleon $s$ are given by

$$
T d s=-\sum_{i} \mu_{i} d Y_{i}
$$

where $T$ is the temperature, $\mu_{i}$ and $Y_{i}$ are the chemical potential and the abundance per nucleon of species $i$, respectively, and the sum runs over all species present. Because photons always and neutrinos generally have zero chemical potentials in nucleosynthetic environments, Eq. (31) becomes

$$
T d s=-\sum_{i}^{\text {nuclear }} \mu_{i} d Y_{i}-\mu_{e} d Y_{e},
$$

where now the sum runs only over nuclear species and where $\mu_{e}$ and $Y_{e}$ respectively refer to the chemical potential of electrons and the abundance per nucleon of net electrons (i.e., electrons in excess of positrons). By charge neutrality,

$$
Y_{e}=\sum_{i} Z_{i} Y_{i}
$$

where $Z_{i}$ is the nuclear charge of species $i$.

The maximum of the entropy per nucleon $s$ (the condition $d s=0$ ) subject to the constraint in Eq. (29) is the most general statistical equilibrium for a nucleosynthetic system. If no constraint is imposed on $Y_{e}$, this statistical equilibrium includes weak equilibrium between neutrons and protons. To find the entropy maximum subject to the constraint in Eq. (29), it is convenient to define a function $f$ such that

$$
f=1-\sum_{i}^{\text {nuclear }} A_{i} Y_{i}
$$

and to introduce a Lagrange multiplier $\lambda$. The problem of finding the entropy maximum subject to conservation of the number of nucleons thus becomes that of finding the abundances $Y_{i}$ such that $d(s-\lambda f)=0$. From Eqs. (31), (32), and (33), one finds that $\lambda=\mu_{n} / k T$, $\mu_{n}=\mu_{p}+\mu_{e}$, and $\mu_{i}=Z_{i} \mu_{p}+\left(A_{i}-Z_{i}\right) \mu_{n}$, where $\mu_{n}$ and $\mu_{p}$ are the chemical potentials of neutrons and protons, respectively. With the appropriate ideal gas expressions, the following equations result:

$$
\begin{aligned}
Y\left(Z_{i}, A_{i}\right)= & G\left(Z_{i}, A_{i}\right)\left[\zeta(3)^{A_{i}-1} \pi^{\left(1-A_{i}\right) / 2} 2^{\left(3 A_{i}-5\right) / 2}\right] \\
& \times A_{i}^{3 / 2}\left(k T / m_{N} c^{2}\right)^{3\left(A_{i}-1\right) / 2} \phi^{1-A_{i}} \\
& \times Y_{p}^{Z_{i}} Y_{n}^{A_{i}-Z_{i}} \exp \left[B\left(Z_{i}, A_{i}\right) / k T\right]
\end{aligned}
$$

and

$$
Y_{p} / Y_{n}=\frac{\rho N_{A} Y_{e}}{2}\left(\frac{2 \pi \hbar^{2}}{m_{e} k T}\right)^{3 / 2} \exp \left(Q_{0} / k T\right) .
$$

In these equations $G(Z, A)$ is the nuclear partition function, $\zeta(3)$ is the Riemann zeta function of argument $3[\zeta(3) \simeq 1.202], m_{N}$ is the nucleon mass, $\phi$ is the photon to nucleon ratio, $N_{A}$ is Avagadro's number, $m_{e}$ is the mass of the electron, $\rho$ is the density, and $Q_{0}=m_{p} c^{2}+m_{e} c^{2}-m_{n} c^{2}$. The photon to nucleon ratio is given by

$$
\phi=\frac{1}{\pi^{2}} \frac{2}{(\hbar c)^{3}} \frac{\zeta(3)(k T)^{3}}{\rho N_{A}} .
$$

With Eqs. (36), (35), (34), and (29), it is possible to determine the abundances given $T$ and $\rho$.

It may seem paradoxical that definite expressions exist for the abundances of the nuclear species in statistical equilibrium. Statistical equilibrium is the condition of maximum entropy and thus of maximum randomness: all allowed macroscopic states, that is, all sets of abundances yielding total energy $E$ and satisfying Eq. (31), are available to the system, and all are equally likely. How then are definite abundances [Eq. (34)] possible? The answer is that the statistical equilibrium abundances in Eqs. (34) and (35) actually represent an average over the ensemble of allowed macroscopic states. The vast majority of the allowed macroscopic states have abundances $\left\{Y_{i}\right\}$ very similar to those in Eqs. (34) and (35). Because of the huge number of allowed macroscopic states in the ensemble, the deviation of the abundances of any macroscopic state from the average is unlikely to be large, and Eq. (34) accurately gives the abundance in equilibrium at any instant in time. This point is not merely pedantic-it is crucial for understanding how order develops during nucleosynthesis.

As Hoyle (1946) and $\mathrm{B}^{2} \mathrm{FH}$ recognized, the time scales present in nucleosynthetic environments may not be sufficiently long to allow weak equilibrium. In this case $Y_{e}$ is slowly varying and may be taken to be instantaneously constant; thus, a new constraint is imposed on 
allowed macroscopic states. This constraint vastly reduces the number of allowed macroscopic states in the ensemble from the number when that constraint is absent. The statistical equilibrium in which $Y_{e}$ is fixed is therefore much more ordered than that for which weak equilibrium holds.

When the entropy is maximized subject to the constraints in Eqs. (29) and (32) the abundances again are given by Eq. (34). Now, however, Eq. (35) is replaced by the requirement that the abundances also satisfy Eq. (32). The abundances for specified $T, \rho$, and $Y_{e}$ may now be found from Eqs. (34), (32), and (28). This particular statistical equilibrium at constant $Y_{e}$ is what most researchers mean when they refer to "nuclear statistical equilibrium," or NSE.

An even more ordered equilibrium in the hierarchy of statistical equilibria is quasiequilibrium (QSE). There exists a whole sequence of possible QSE's, but the least restricted arises from the additional possible constraint that $Y_{h}$, the number of heavy nuclei (those nuclei with $A \geqslant 12$ ), is instantaneously fixed. This equilibrium is in fact realized in nature because the reactions assembling heavy nuclei can be much slower than the other reactions occurring in the system (except those changing $\left.Y_{e}\right) . Y_{h}$ is calculated as

$$
Y_{h}=\sum_{i, A_{i} \geqslant 12}^{\text {nuclear }} Y_{i}
$$

This QSE is even more ordered than NSE because of the further reduction in the number of allowed macroscopic states in the ensemble. Meyer, Krishnan, and Clayton (1997) have found the abundances in this QSE by maximizing the entropy subject to the constraints of constant nucleon number, $Y_{e}$, and $Y_{h}$, that is, Eqs. (29), (32), and (37). The result is that the abundance of each heavy nucleus $(A \geqslant 12)$ is given by

$$
Y(Z, A)=e^{\mu_{h} / k T} R_{p}^{Z} R_{n}^{A-Z} Y^{\mathrm{NSE}}(Z, A),
$$

where $R_{p}=Y_{p} / Y_{p}^{\mathrm{NSE}}$ and $R_{n}=Y_{n} / Y_{n}^{\mathrm{NSE}}$ measure the overabundances of free neutrons and protons in the QSE relative to NSE and where $Y^{\mathrm{NSE}}(Z, A)$ is the NSE abundance of nucleus $(Z, A)$. Meyer et al. (1997) were able to interpret $\mu_{h}$ as the chemical potential of heavy nuclei in the system. The abundances in QSE for specified $T, \rho, Y_{e}$, and $Y_{h}$ may now be found from Eqs. (38), (37), (32), and (29). It is also necessary in solving for QSE to solve for the NSE abundances via Eq. (34).

More restricted QSE's arise from the possibility that the total number of nuclei in different ranges of atomic number or mass number might be slowly varying. For example, it may be that not only are the total number of heavy nuclei only slowly changing with time, but also the number $Y_{h 1}$ of nuclei with $10 \leqslant Z \leqslant 20$ and the number $Y_{h 2}$ with $21 \leqslant Z \leqslant 30$ might separately be nearly constant in time. In this case, there arises an additional constraint on the equilibrium, and the system is even further ordered. The abundances are given by equations similar to Eq. (28), but now with chemical potentials $\mu_{1}$ and $\mu_{2}$ appropriate for the separate equilibrium clusters.

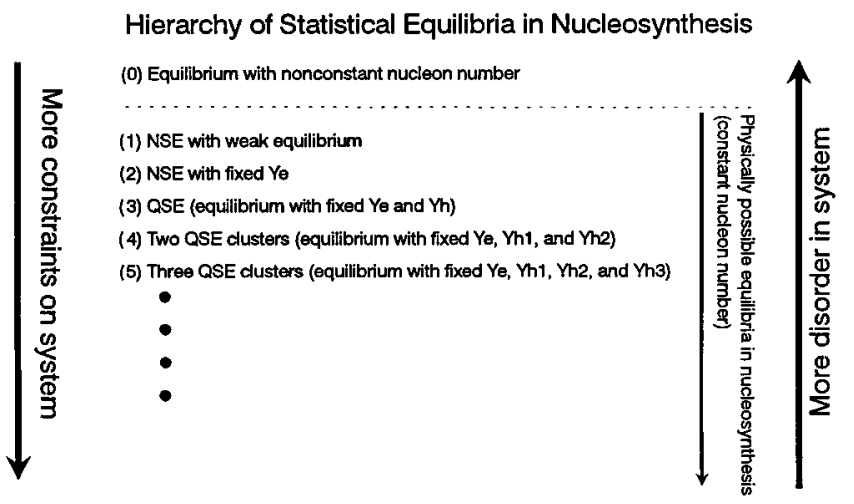

FIG. 26. The hierarchy of possible statistical equilibria in nucleosynthetic systems. The statistical equilibria are conditions of maximum entropy subject to certain constraints. The smaller the number of constraints present, the more disordered the system is and the higher up the hierarchy the equilibrium sits. All possible statistical equilibria in nucleosynthetic systems have the constraint of constant nucleon number and thus lie below the dotted line. Since one or a few key time scales control the evolution of the system in many cases, nature is able to approximate these equilibria to high accuracy.

Figure 26 summarizes the hierarchy of statistical equilibria possible in nucleosynthesis. At the very top, the only constraint is that the energy of the system is $E$. There is no constraint on the number of nucleons in the system. As argued above, such an equilibrium is impossible in any nucleosynthetic environment; therefore, only statistical equilibria lying below the dotted line in Fig. 26 are possibly relevant to nucleosynthesis. The highest relevant statistical equilibrium is NSE with weak equilibrium. Adding constraints leads to a descent of the hierarchy and an increase in order in the system. On the other hand, temporal evolution may be such as to ascend the hierarchy by removing constraints.

A system in a dynamical environment is unlikely to precisely achieve any of the statistical equilibria shown in Fig. 26. This depends on the interplay of many nuclear reactions, all occurring on different time scales. Nevertheless, many systems do nearly manage to maximize the entropy subject to one or a few key time scales. This means that the concept of a hierarchy of statistical equilibria will be helpful in understanding these systems. Such is the case with the iron-group nuclides.

\section{A brief history of the ideas of iron-group element synthesis}

For a valuable chronicle of this major intellectual step, the reader is referred to Clayton (1996).

$\mathrm{B}^{2} \mathrm{FH}$ ascribed synthesis of the iron-group isotopes to the $e$ process, by which they meant the "equilibrium" process. They envisioned NSE occurring near $Y_{e}=26 / 56=0.464$ in order that ${ }^{56} \mathrm{Fe}$ be made as itself in correct proportion to other $\mathrm{Fe}$ isotopes. In fact, they fixed the temperature and the ratio $Y_{p} / Y_{n}$ of free protons to neutrons in their calculations. Their argument for fixed $Y_{p} / Y_{n}$ was that once the system falls out of 
weak equilibrium, the weak reactions will be slow and will change $Y_{p} / Y_{n}$ very slowly throughout the rest of the expansion and cooling of the material. This is actually a bit of a shaky assumption. It is true that the slowness of the weak reactions will keep $Y_{e}$ nearly constant, but there is no guarantee, for example, that free neutrons would not capture onto nuclei more quickly than free protons. This would certainly alter $Y_{p} / Y_{n} . \mathrm{B}^{2} \mathrm{FH}$ recognized this difficult point and called for detailed astrophysical calculations to get $Y_{p} / Y_{n}$ correct throughout a stellar explosion. Much of the progress since 1957 has come in precisely this quest.

$\mathrm{B}^{2} \mathrm{FH}$ obtained their best fit to the solar abundances of iron-group nuclei in the mass range $50 \leqslant A \leqslant 62$ for a temperature $T_{9}=3.78$ and a ratio $Y_{p} / Y_{n} \approx 300$. With the assumption of weak equilibrium Eq. (36), this implies a density of $\rho \approx 1.5 \times 10^{5} \mathrm{~g} / \mathrm{cm}^{3}$. Their results included some estimates for freeze-out corrections.

It is interesting to note that the conditions $\mathrm{B}^{2} \mathrm{FH}$ found best fit the solar system's abundances of Fe-group nuclei, corresponding at $T_{9}=3.78$ to an entropy per baryon $s / k \approx 50$, which is fairly large. By comparison, modern supernova calculations show that Fe-group nuclei are produced in Type Ia supernovae at entropies $s / k \leq 1$ and in Type II supernovae at entropies $s / k \approx 10$.

A fundamental obstacle to the synthesis of ${ }^{56} \mathrm{Fe}$ in stellar evolution was that, in a sequence of burning phases, the synthesis of ${ }^{28} \mathrm{Si}$ would precede the $e$ process. Direct conversion of ${ }^{28} \mathrm{Si}$ nuclei into ${ }^{56} \mathrm{Fe}$ is difficult because (1) the Coulomb barriers for interactions of ${ }^{28} \mathrm{Si}$ with each other are too large and (2) $Y_{e}$ would have to drop from $\sim 0.5$ for ${ }^{28} \mathrm{Si}$ to $\sim 0.464$ for ${ }^{56} \mathrm{Fe}$. The correct answer would turn out to be what we now call silicon burning.

First, network calculations showed that ${ }^{28} \mathrm{Si}$ burned into ${ }^{56} \mathrm{Ni}$ (Truran, Arnett, and Cameron, 1967). This is because $Y_{e}$ changes little during the burning of silicon and ${ }^{56} \mathrm{Ni}$ is the most bound nucleus with $Y_{e}$ near 0.5 (see Fig. 24). The ${ }^{56} \mathrm{Ni}$ subsequently decays into ${ }^{56} \mathrm{Fe}$ since the latter is a more tightly bound nucleus, and a profoundly important question became whether that decay occurred during presupernova evolution or after ejection of the matter. A second crucial point is the way silicon burning actually proceeds. Because the reaction ${ }^{28} \mathrm{Si}+{ }^{28} \mathrm{Si} \rightarrow{ }^{56} \mathrm{Ni}$ does not occur directly, some ${ }^{28} \mathrm{Si}$ must break down into alpha particles which then capture onto the remaining ${ }^{28} \mathrm{Si}$. The effect is to decrease the number of heavy nuclei in accordance with the dictates of NSE. The crucial point recognized by Bodansky et al. (1968) is that, as the ${ }^{28} \mathrm{Si}$ nuclei are stripped down by $(\gamma, \alpha)$, additional reactions such as ${ }^{20} \mathrm{Ne}(\gamma, \alpha){ }^{16} \mathrm{O}$ increase the abundance of alphas. This leads to an increase in the rate of $(\alpha, \gamma)$ reactions which impede the ${ }^{28} \mathrm{Si}$ disintegration flow and slow the decrease in the number of heavy nuclei. At the same time, reverse reactions between $A=28$ and $A=58$ come into a steady-state balance. Thus, while most reactions occur quickly and drive the system toward equilibrium, the equilibrium achieved is not NSE because the abundance of heavy nuclei is not that demanded by NSE. The equilibrium achieved is rather QSE, the third of the possible nucleosynthesis statistical equilibria in the hierarchy in Fig. 26. Only for long times can $Y_{h}$ reach its NSE value and allow the system to ascend the hierarchy further. The agreement between the solar system Fe-group abundances and results of QSE and network calculations showed that QSE silicon burning was important in the synthesis of the bulk of the solar system's ${ }^{56} \mathrm{Fe}$. It also offered the hope of testing this theory through observation of gamma rays emitted by specific nuclei (Clayton et al., 1969). The possibility of confirming nucleosynthesis in this way was not foreseen by $\mathrm{B}^{2} \mathrm{FH}$.

Woosley et al. (1973) provided further refinements in ideas about equilibrium synthesis of Fe-group nuclei. Most importantly, they considered higher entropies in silicon (and oxygen) burning, such as those that might be established by dissipation of a supernova shock wave in the Si shell. The interesting result was that, unlike in calculations of lower-entropy expansions of material from high temperature and density, the final asymptotic mass fraction of free alphas was not negligibly small. Woosley et al. (1973) termed this the "alpha-rich freeze out." The astronomical relevance is that synthesis of ${ }^{56} \mathrm{Ni}$ in Type II supernovae appears to occur via this process. Also of significance is the fact that an alpha-rich freeze out naturally produces the radioactive species ${ }^{44} \mathrm{Ti}$ in sufficiently high abundance to account for natural ${ }^{44} \mathrm{Ca}$.

The second interesting point was that Woosley et al. (1973) studied in detail the approach both to QSE and to NSE. The initial state of the system in these alpharich freeze-out scenarios, the ${ }^{28} \mathrm{Si}$-rich shell of a massive star, is a highly ordered state because nearly all nucleons are locked into ${ }^{28} \mathrm{Si}$ nuclei. As a shock wave passes through this zone during the supernova explosion, the material is heated. Normal silicon burning occurs as some ${ }^{28} \mathrm{Si}$ nuclei are stripped down into alphas which then are captured by other Si nuclei. Eventually small clusters of neighboring isotopes come into equilibrium under exchange of $n$ 's, $p$ 's, and $\alpha$ 's. This type of statistical equilibrium is low in the hierarchy of statistical equilibria, but it is more disordered than the original state. In the subsequent evolution, the different equilibrium clusters begin to merge, first to the $A=28$ to $A=42$ region, later up to the $A=58$. This reduction in time in the number of constraints on the system enable the equilibria to climb up the hierarchy of statistical equilibria, with the system becoming more disordered. Given sufficient time, the clusters merge into a single large equilibrium cluster containing almost all of the heavy isotopes, but the abundance of heavy nuclei still differs from that desired by NSE. In many cases in Type II supernova explosions, this is the farthest up the statistical equilibrium hierarchy (and the most disordered) the system gets during explosive silicon burning because of the short hydrodynamical timescales. As the system expands and cools, the evolution reverses in the sense that larger clusters break up into smaller ones, the system descends the hierarchy of statistical equilibria, and order reap- 
pears. For a recent discussion of the evolution of QSE clusters in silicon burning, see Hix and Thielemann (1996).

Natural systems do reach the higher ranks of the statistical equilibrium hierarchy. The cores of massive stars can evolve nearly all the way to the top (NSE with weak equilibrium) because of the relatively long time scales and high densities in presupernova evolution. Interestingly, ${ }^{56} \mathrm{Fe}$ can there dominate the abundance, just as $\mathrm{B}^{2} \mathrm{FH}$ envisioned. Were this material (and only this material) simply ejected, ${ }^{56} \mathrm{Fe}$ would indeed be made by the $e$ process! In fact, calculations show that little of this matter actually gets ejected during the explosion. Most of it remains in the compact remnant. That little which is ejected is heated to high temperatures and undergoes an alpha-rich freeze out (Thielemann et al., 1990; Woosley and Hoffman, 1991, Woosley and Hoffman, 1992). Silicon burning in the $\mathrm{Si}$ and $\mathrm{O}$ shells makes the bulk of the ${ }^{56} \mathrm{Fe}$, via ${ }^{56} \mathrm{Ni}$, ejected by the massive star.

The nucleosynthesis in Type Ia supernovae (that is, deflagration or detonation of carbon-oxygen white dwarf stars) also can ascend to near the top of the statistical equilibrium hierarchy. The bulk of the matter in the explosion can certainly achieve an NSE (e.g., Thielemann et al., 1986; Woosley and Weaver, 1994) having $Y_{e}=0.5$. Thus there is copious production of ${ }^{56} \mathrm{Ni}$. In the cores, the density may even get high enough that the material approaches weak equilibrium. In such a case, electron captures occur and $Y_{e}$ drops so that more neutron-rich species (including ${ }^{56} \mathrm{Fe}$ !) may be produced directly. It is here that nature may produce and even eject ${ }^{56} \mathrm{Fe}$ by $\mathrm{B}^{2} \mathrm{FH}$ 's $e$ process! In certain rare Type Ia's, the central density may even be high enough that the approach to weak equilibrium drives $Y_{e}$ low enough to produce rare iron-group isotopes like ${ }^{48} \mathrm{Ca},{ }^{50} \mathrm{Ti}$, and ${ }^{54} \mathrm{Cr}$ (Woosley et al., 1995; Meyer et al., 1996; Woosley, 1996).

While $\mathrm{B}^{2} \mathrm{FH}$ correctly surmised that the bulk of the Fe-group isotopes in the solar system were made in equilibrium processes, they also found that the $s$ process had to be responsible for the synthesis of some of the iron-group nuclides. Work since 1957 has confirmed this to be the case (e.g., Peters et al., 1972; Lamb et al., 1977; Baraffe et al., 1992). In this process, seed iron-group nuclei from previous generations of stars are exposed to a flux of neutrons. This shifts the abundances over to the more neutron-rich of the iron-group isotopes. The weak $s$ process most likely occurs in the helium burning cores of massive stars where $(\alpha, n)$ reactions on ${ }^{13} \mathrm{C}$ and ${ }^{22} \mathrm{Ne}$ can generate a sufficient supply of neutrons. For more details, the reader should turn to Secs. IX and X of this review.

\section{Significance for astrophysics}

With the key ideas of iron-group nucleosynthesis more or less clear since the early 1970's, the recent work has centered on obtaining improved yield estimates for these nuclei and on exploring their significance for astro- physics. This subsection provides a very brief discussion of these aspects of synthesis of the iron-group elements.

Advances in computer technology have fostered great increases in the sophistication of astrophysical irongroup yield estimates. The yields are now computed in postprocessing calculations using detailed nuclear networks and hydrodynamics from realistic stellar models. This is true for both Type Ia (e.g., Thielemann et al., 1986; Woosley and Weaver, 1994) and Type II (e.g., Thielemann et al., 1996; Woosley and Weaver, 1995) supernova models (see also Arnett, 1996). Further advances will come as the remaining uncertainties in the models are dealt with realistically. In Type Ia models, key uncertainties affecting the Fe-group yields include the correct treatment of the nuclear flame speed and acceleration (e.g., Woosley and Weaver, 1994; Niemeyer and Woosley, 1997; also Arnett, 1996) and the rates for electron capture on the Fe-group isotopes (e.g., Fuller et al., 1980, 1982a, 1982b, 1985; Aufderheide et al., 1994). Stellar spectroscopists hope to clarify the Ia mechanism through observations of time-dependent atomic lines. In Type II models, key uncertainties include the location of the mass cut that separates matter ejected from that that stays in the remnant (e.g., Weaver and Woosley, 1993), the role of convection near the nascent neutron star (e.g., Herant et al., 1992), and the correct treatment of the explosion shock (e.g., Aufderheide et al., 1991).

Astronomical observations will play a crucial role in determining the improvements needed in theoretical yield estimates. Both gamma line astronomy and light curves rely on the key fact that so many of the irongroup species had radioactive progenitors. Observations of supernova light curves, powered in large measure by the decay of the radioactive iron-group species ${ }^{56} \mathrm{Ni}$ and ${ }^{56} \mathrm{Co},{ }^{57} \mathrm{Co}$, and ${ }^{44} \mathrm{Ti}$, provide important estimates of the yields of these isotopes (e.g., Woosley et al., 1989; Suntzeff $e$ al., 1992), replacing the ${ }^{254} \mathrm{Cf}$ hypothesis of $\mathrm{B}^{2} \mathrm{FH}$. Long standing theoretical expectations were confirmed, with surprises, by observations of $\gamma$ rays from the decay of these radioactive species in the debris of individual supernovae: ${ }^{56}$ Co (e.g., Leising and Share, 1990) and ${ }^{57}$ Co (Kurfess et al., 1992) from SN 1987A; ${ }^{44} \mathrm{Ti}$ (Dupraz et al., 1996; The et al., 1996) from Cas A. While the observations of the $\mathrm{Ni}$ isotopes are in fairly good agreement with theoretical predictions (agreement within $\sim 50 \%$ ), the differences present valuable challenges that will ultimately lead to improved supernova models. Of further astronomical interest, ${ }^{56} \mathrm{Co}$ and ${ }^{44} \mathrm{Sc}$ also eject positrons which may be the dominant source of the 511 $\mathrm{keV} \gamma$ ray line and positronium continuum flux from the Galactic plane (e.g., Lingenfelter and Ramaty, 1989).

Astronomical observations of iron-group isotopes may also provide information on the Galaxy's history. For example, stellar observations of iron and oxygen in stars of varying age (e.g., Peterson et al., 1990, Gratton and Sneden, 1991) provide valuable constraints on the growth of elemental abundances during the Galaxy's history. Detailed models of Galactic chemical evolution (e.g., Timmes et al., 1995) must confront these con- 
straints. Among the pieces of information such a confrontation will yield is the relative contribution of Type I and Type II supernovae to the solar system's budget of ${ }^{56} \mathrm{Fe}$. The current models seem to suggest they contributed roughly equally (e.g., Timmes et al., 1995). Other valuable information on the Galaxy's history comes from gamma ray astronomy. Gamma ray observations of the relatively long-lived iron-group radioactivity ${ }^{60} \mathrm{Fe}$ provide useful information on the Galactic supernova rate over the last million years (Leising and Share 1994).

Another area of astronomy on which the iron-group isotopes may have a great impact is in solar system studies. Strong evidence exists that the iron-group radioactivities ${ }^{53} \mathrm{Mn}$ and ${ }^{60} \mathrm{Fe}$ were alive in small part at the time of formation of solar system solid bodies (Harper and Wiesmann, 1992; Shukolyukov and Lugmair, 1993). This information constrains the last nucleosynthesis events contributing to the solar system's supply of isotopes (e.g., Harper, 1996) and the time scales of solidbody formation in the early solar system (e.g., Shukolyukov and Lugmair, 1993). Correlated anomalies of the neutron-rich iron-group species ${ }^{48} \mathrm{Ca},{ }^{50} \mathrm{Ti}$, and ${ }^{54} \mathrm{Cr}$ in calcium-aluminum-rich inclusions in meteorites (e.g., Loss and Lugmair, 1990; Volkening and Papanastassiou, 1990) strongly suggest these isotopes retain some memory of their site of nucleosynthetic origin, while endemic anomalies in ${ }^{50} \mathrm{Ti}$ in all classes of carbonaceous meteorites (Niemeyer, 1988) hint at an inhomogeneous distribution of this isotope in the interstellar dust that comprised the original building material of the solar system. Especially noteworthy are the huge ${ }^{44} \mathrm{Ca}$ excesses found in graphite and silicon carbide $\mathrm{X}$ grains in primitive meteorites (e.g., Hoppe et al., 1996). The excesses undoubtedly come from in situ decay of ${ }^{44} \mathrm{Ti}$ (half life of 47 years) and provide proof that these grains condensed in outflowing supernova debris. In $1957, \mathrm{~B}^{2} \mathrm{FH}$ could only have dreamed of having such tangible evidence of element formation in stars!

\section{CARBON STARS: WHERE THEORY MEETS OBSERVATIONS}

\section{A. Prologue}

To this observer the most remarkable aspect of the classic tale "Synthesis of the Elements in Stars" told by Burbidge, Burbidge, Fowler, and Hoyle (1957, here $\mathrm{B}^{2} \mathrm{FH}-$ of course) is, perhaps, their confidence in theoretical ideas as revealed by the almost complete lack of appeal to observations of the compositions of the stars to whom element synthesis was attributed. In large part, the omission of observational data on the compositions of stellar atmospheres reflected, however, the simple fact that in 1957 the available quantitative data were sparse in the extreme. Fortunately, key clues to the nuclear astrophysics of various kinds of carbon stars were obtainable from qualitative inspection of stellar spectra: for example, the presence of ${ }^{13} \mathrm{C}$-containing molecules suggested exposure of material to a hydrogenburning region; furthermore, the presence of technetium in some cool carbon stars implied, as in the S-type oxygen-rich stars, recent or even continuing operation of the $s$ process. Curiously, $\mathrm{B}^{2} \mathrm{FH}$ were extremely cautious in their sifting of spectroscopic clues for evidence of helium burning. In contrast to several lines of evidence cited in support of hydrogen burning in carbon and other stars, $\mathrm{B}^{2} \mathrm{FH}$ described the evidence for helium burning as "less direct" and among a few snippets of such evidence did not cite carbon stars.

In 1957, origins of carbon stars were shrouded in mystery. Bidelman (1956) opened the review "The Carbon Stars-An Astrophysical Enigma" with the declaration that the stars have "posed a vexing astrophysical problem" since Father Secchi's identification of the cool carbon stars in the previous century. Forty years on, problems remain but for some classes of carbon stars observation and theory have converged on an evolutionary scenario in which nuclear astrophysics occurring in a stellar interior and spectroscopy of an atmosphere are related by a complete model of a stellar interior, envelope, and atmosphere. In this brief review, I sketch the convergence of theory and observation, as well as the lack of convergence that remains for some classes of carbon stars. I do not discuss at all the contributions of the carbon stars to the chemical evolution of the Galaxy, as Gustafsson and Ryde (1997) give a comprehensive review of that topic.

\section{B. Carbon stars-An observer's view}

\section{What is a carbon-rich star?}

An observer's working definition is that "carbon star" is certainly an appropriate label when the ratio of carbon to oxygen by number of atoms exceeds unity, here $\mathrm{C} / \mathrm{O}>1$.

Thanks to the preeminent role of the $\mathrm{CO}$ molecule in controlling the partial pressures of carbon and oxygen at low temperatures, spectra of oxygen $(\mathrm{C} / \mathrm{O}<1)$ and carbon $(\mathrm{C} / \mathrm{O}>1)$ rich cool stars are readily distinguishable at even low spectral resolution: optical spectra of oxygen stars are dominated by metal oxide (e.g., TiO, YO, ZrO) bands, and spectra of carbon stars by bands of carbon containing molecules, principally $\mathrm{C}_{2}$ and $\mathrm{CN}$. (For $\mathrm{C} / \mathrm{O}$ $<1$, the presence of oxides other than $\mathrm{TiO}-$ i.e., $\mathrm{YO}$ and $\mathrm{ZrO}$-implies a $\mathrm{C} / \mathrm{O}$ ratio close to unity. Such molecular features are a defining mark of the S-type stars.)

At higher temperatures when the influence of the $\mathrm{CO}$ molecule is weak or unimportant, mildly carbon-rich stars may go unnoticed by mere inspection of low resolution spectra. I have in mind here the case of the classical barium giants discovered by Bidelman and Keenan (1951). These G- and K-type giants with enhanced CH and $\mathrm{C}_{2}$ bands are presumably carbon enriched relative to normal giants of the same type. CO still has an influence on the partial pressures of carbon and oxygen but a detailed analysis is needed to show if $\mathrm{C} / \mathrm{O}$ exceeds unity. Such analyses show that Ba II stars of roughly solar metallicity have $\mathrm{C} / \mathrm{O}<1$ but $\mathrm{C} / \mathrm{O}$ is higher than in normal giants (Tomkin and Lambert, 1979; Smith, 1984; Barbuy 
et al., 1992). For the $\mathrm{CH}$ stars discovered by Keenan (1942) which are metal-poor halo counterparts of the Ba II stars, Vanture (1992a, 1992b) found C/O to run from just in excess of 1 to 8 . Clearly, the $\mathrm{CH}$ stars deserve the appellation "carbon star" but, in a strict sense, the Ba II stars do not. Since it is now evident that the two groups have a common evolutionary origin, I consider Ba II stars here with stars that do satisfy the criterion $\mathrm{C} / \mathrm{O}>1$.

For the several classes of carbon stars alluded to in the preceding paragraphs, it appears that they possess a roughly normal hydrogen abundance, which implies a normal helium abundance. Certainly, hydrogen is seen in the spectra either as the Balmer lines in absorption or emission or as lines of a $\mathrm{H}$-containing molecule such as $\mathrm{CH}$. Bidelman (1956) included several categories of very hydrogen-poor stars with strong spectral features of carbon among the carbon stars. $\mathrm{B}^{2} \mathrm{FH}$ also refer to these stars: "Some rare specimens among the carbon stars have weak or absent $\mathrm{CH}$ bands and hydrogen lines." For these stars, there is the possiblity that carbon is enriched in the sense that the carbon mass fraction is many times the low value it has in normal stars and even in the carbon stars of normal hydrogen abundance. A few of the hydrogen deficient stars are cool enough to be sure that $\mathrm{C} / \mathrm{O}>1$ but others are too warm for the $\mathrm{C} / \mathrm{O}$ ratio to be inferred except by a rather detailed analysis. For lack of space I do not consider hydrogen deficient stars such as the $\mathrm{R}$ Coronae Borealis variables, the hydrogen deficient cool carbon stars, and the extreme helium hot stars.

\section{What makes a carbon-rich star?}

At the present time, the interstellar medium is oxygen rich. Models of galactic chemical evolution (e.g., Timmes et al., 1995) predict that the interstellar medium has always been oxygen rich thanks to a greater production of oxygen relative to carbon. Oxygen (here, ${ }^{16} \mathrm{O}$ ) is synthesized by massive stars. Carbon (here, ${ }^{12} \mathrm{C}$ ) is synthesized not only by massive stars but also by lower mass stars. Prantzos et al. (1996) summarize theoretical evidence on the stellar sites for the synthesis of all stable isotopes of carbon, nitrogen, and oxygen. On the assumption that the surface abundances of carbon and heavier elements for an unevolved or main sequence star are identical to the abundances in the parental interstellar cloud, all stars began life with oxygen-rich atmospheres. For the sun and stars of approximately solar metallicity, the $\mathrm{C} / \mathrm{O}$ ratio is about 0.6 . Analyses of main sequence stars show that the $\mathrm{C} / \mathrm{O}$ ratio in metal-poor $([\mathrm{Fe} / \mathrm{H}] \leqslant-1)$ stars is about $60 \%$ lower than the solar value (Tomkin et al., 1995). Models of galactic chemical evolution can account for this drop in the $\mathrm{C} / \mathrm{O}$ ratio. In short, carbon stars are not born but are transformed oxygen-rich stars.

The galactic experience as revealed by local disk and halo stars is possibly not universal as to location and age of a stellar system. Since considerable production of carbon but not of oxygen is provided by low mass stars, the $\mathrm{C} / \mathrm{O}$ ratio depends on the history of star formation and, in particular, on the relative numbers of high and low mass stars. If formation of high $\left(M>10 M_{\odot}\right)$ mass stars is consistently suppressed relative to low mass stars in a particular environment, the $\mathrm{C} / \mathrm{O}$ ratio will evolve to higher values than the present value and may well exceed unity. No such regions have been identified, as yet.

Certainly, oxygen-rich main sequence stars are considered the norm. Then, the question "What makes a carbon-rich star?" becomes "How is an oxygen-rich star transformed to a carbon-rich star?" Existence of carbonrich stars might be accounted for in three broad ways: (i) the stars formed from an interstellar cloud locally enriched in carbon (relative to oxygen); (ii) nucleosynthesis followed by internal processes (diffusion, mixing, mass loss) transformed the star from oxygen-rich to carbon-rich; and (iii) carbon was transferred to the star after its birth to increase the surface $\mathrm{C} / \mathrm{O}$ ratio.

Possibility (i) seems unlikely but data on the composition of dark clouds, the sites of ongoing or prospective star formation, are too sparse for its exclusion on observational grounds. Limited data on the carbon abundance of $\mathrm{H}$ II regions in conjunction with ample measurements of their oxygen abundance suggests that the $\mathrm{C} / \mathrm{O}$ ratio is close to the solar value and never exceeds unity. Since low mass stars eject lots of carbon at low speeds into the interstellar medium, it may be possible for pockets within clouds to be made carbon rich and to have eluded observers.

Possibility (ii) provides opportunities for transforming evolved stars with $\mathrm{C} / \mathrm{O}<1$ to stars with $\mathrm{C} / \mathrm{O}>1$ but opportunities for transforming main sequence stars seem limited. Among the chemically peculiar A and B-type main sequence stars is one class-the $\mathrm{SrCrEu}$ stars-with members that are carbon-rich by the measure $\mathrm{C} / \mathrm{O}>1$ (Roby and Lambert, 1990). These stars for which elemental segregation driven by the combination of gravitational settling and radiation pressure is a likely explanation will not be discussed here, as they are not generally considered under the rubric of "carbon stars." Surface abundances of carbon and oxygen will be affected by internal mixing only if it extends into regions that are experiencing or have experienced nuclear reactions. Since hydrogen-burning reactions at the interior temperatures of main sequence stars reduce the $\mathrm{C} / \mathrm{O}$ ratio, mixing cannot result in a carbon-rich star. Mixing occurs in massive main sequence stars where it is probably driven by rapid rotation and revealed by the presence of carbon-poor but nitrogen-rich stars (Lyubimkov, 1993; Gies and Lambert, 1992). Hydrogen burning is followed by helium burning with a product that has $\mathrm{C} / \mathrm{O}>1$. Wolf-Rayet stars of the WC types are carbon stars by the definition $\mathrm{C} / \mathrm{O}>1$ but are not considered here. In the case of the WC stars, severe mass loss exposes the carbon-rich layers that were at a prior time deep in the star and exposed to helium burning.

For evolved stars, possibility (ii) offers a richer variety of possibilities. In particular, low $\left(M<10 M_{\odot}\right)$ mass stars evolved beyond helium-core burning experience heliumshell flashes (thermal pulses) which, in some calculations, are predicted to be followed by extension of the 
giant's deep convective envelope into the top of the (now quiescent) helium shell. This extension or third dredge-up occurs repeatedly and brings carbon and $s$-process products into the photosphere. (I. Iben, Jr., discusses this phase of AGB evolution in Sec. III.) There is no doubt that the third dredge up in AGB stars is a primary process for converting oxygen-rich to carbonrich giants. Questions remain about, for example, the efficacy of the third dredge up in low mass stars, but it may be claimed that theory and observation have converged for carbon-rich AGB stars (N-type stars-see below), as well as for the carbon-rich stars produced by mass transfer across a binary system (see below). There remain speculative possibilities for internal mixing leading to a carbon-rich star. Invocation of the helium core flash in low mass stars is one such possibility. Severe mass loss is another possibility. Such speculations are aired in connection with carbon-rich stars for which theory and observation have yet to converge, as in the case of the R stars (see below).

Mass transfer [possibility (iii)] is the accepted mechanism for the formation of several classes of main unevolved and evolved carbon stars (barium dwarfs, $\mathrm{CH}$ subgiants, barium and $\mathrm{CH}$ giants). In this case, a star evolves to become a cool carbon star (N-type AGB star, see below) and transfers mass to its less evolved companion, which is converted to a carbon-rich star. The companion remains as a white dwarf orbiting the new carbon star. Mass transfer from an AGB star to a companion occurs only in a binary of wide separation. Binaries of closer separation experience mass transfer before the more massive star has become a large AGB star. Perhaps, in certain cases, the two stars develop a common envelope, coalesce, and the single star that results has experienced such extensive mixing that carbon from the core of the previously more evolved star appears at the surface.

\section{The principal types of carbon-rich stars}

In the following sections, I highlight some of the properties of several classes of galactic carbon stars. In general, the highlights are restricted to observations that are particularly pertinent to establishing the evolutionary state of the stars, i.e., luminosity, and aspects of the chemical compositions.

\section{a. R-type carbon stars}

The Harvard system of spectral classification introduced types $\mathrm{R}$ and $\mathrm{N}$ for cool stars with strong bands of $\mathrm{CN}$ and $\mathrm{C}_{2}$. This separation remains useful. The $\mathrm{R}$ stars, which are systematically warmer than the $\mathrm{N}$ stars, are, as judged by temperature and luminosity, $\mathrm{K}$ giants with enhanced bands of $\mathrm{CN}$ and $\mathrm{C}_{2}$. The $\mathrm{N}$ stars are cooler and more luminous. A further broad division is helpful: the $\mathrm{N}$ stars may be divided into those that do and do not show strong spectral features attributable to the isotope ${ }^{13} \mathrm{C}$ : bands of ${ }^{13} \mathrm{CN}$ and ${ }^{12} \mathrm{C}^{13} \mathrm{C}$ or even ${ }^{13} \mathrm{C}_{2}$ are often distinguishable at low to moderate spectral resolution from their parent bands of the more abundant ${ }^{12} \mathrm{C}$ iso- tope. Stars with strong ${ }^{13} \mathrm{C}$ bands are termed J-type. A small fraction of N-type stars are of the J-type. All or almost all of the R-type stars qualify for the designation of J-type. Thus observers challenge theorists to provide an evolutionary scenario for three types of cool carbon giant stars: $\mathrm{N}, \mathrm{N}(\mathrm{J})$, and $\mathrm{R}$. In broad terms, observations and theory have met and reached agreement about the $\mathrm{N}$ stars: asymptotic giant branch (AGB) stars in which the third dredge up (Iben, this review) has converted an oxygen-rich to a carbon-rich envelope. By contrast, the $\mathrm{R}$ stars are unexplained by theory despite more extensive data on their chemical compositions than are available for the $\mathrm{N}$ stars.

A sample of 11 early R stars was analyzed by Dominy (1984) from high-resolution optical and infrared spectra. His abundance analysis provided the following results that an evolutionary scenario must explain:

(1) Metallicity. The stars are of approximately solar metallicity: $[\mathrm{Fe} / \mathrm{H}]$ ranges from +0.2 to -0.6 .

(2) Lithium. Lithium was not detected. It appears that $\mathrm{R}$ and normal $\mathrm{K}$ giants are not distinguishable by their lithium abundances.

(3) $C, N$, and $O$. The $\mathrm{R}$ stars are carbon stars in the sense that $\mathrm{C} / \mathrm{O}>1$ : Dominy gave $\mathrm{C} / \mathrm{O}$ ratios in the range $1-3$. The oxygen abundance is normal. Nitrogen was markedly enhanced: $[\mathrm{N} / \mathrm{Fe}]=0.7$ where $[\mathrm{N} / \mathrm{Fe}]=0.3$ is characteristic of normal $\mathrm{K}$ giants. The ${ }^{12} \mathrm{C} /{ }^{13} \mathrm{C}$ ratios of the $\mathrm{R}$ stars are in the range $4-15$ for a mean value of $7 \pm 3$.

(4) $s$ process. The $s$-process elements are not significantly enhanced: $[s / \mathrm{Fe}]=0.25 \pm 0.18$. Certainly, the $s$-process abundances are not at the level that is characteristic of Ba II stars.

These results imply that carbon from a heliumburning region has been added to the envelope after substantial exposure to hot protons that convert some of the ${ }^{12} \mathrm{C}$ to ${ }^{13} \mathrm{C}$ and ${ }^{14} \mathrm{~N}$. The stars are too low in luminosity for the addition to have occurred as part of a third dredge-up in the R star itself. Dominy speculated that the helium core flash led to extensive mixing. Since all low $\left(M<2.3 M_{\odot}\right)$ mass stars experience a helium core flash but $\mathrm{R}$ stars are rare among the $\mathrm{K}$ giants (i.e., no $\mathrm{R}$ stars in the Bright Star Catalogue, which contains more than a thousand $\mathrm{K}$ giants), a special condition must be sought that ensures that, in just a few cases, the helium core burning giant is a $\mathrm{R}$ rather than a normal $\mathrm{K}$ giant. Dominy suggested a rapidly rotating core might induce mixing and create a R star. That the helium core flash is the agent creating $\mathrm{R}$ stars has yet to be demonstrated theoretically. Theorists appear to consider the flash to be benign (i.e., mixing does not result). Perhaps recent startling advances in numerical hydrodynamics may be applied to this problem.

In light of the success of the mass transfer hypothesis at explaining the $\mathrm{Ba}$ II and $\mathrm{CH}$ stars, suspicions have surely lingered that the $\mathrm{R}$ stars might also be binaries in which the nucleosynthesis occurred in a luminous highly evolved companion star (now extinct) and transferred to the less evolved $\mathrm{R}$ star. This idea has now been put effectively to rest by the radial velocity survey of $\mathrm{R}$ stars 
of McClure (1997). The remarkable result of his longterm survey is that, in a sample of $22 \mathrm{R}$ stars, "no evidence for binary motion has been detected in any of them" while $20 \%$ of normal $\mathrm{K}$ giants are spectroscopic binaries. McClure suggests that R stars may result from coalescence of two stars with coalescence providing the trigger for mixing at the helium core flash. One might expect coalescence to result in a rapidly rotating $\mathrm{R}$ star as orbital angular momentum is transferred to the coalesced pair but, as McClure notes and Dominy's spectra clearly show, there is no evidence that the R stars are more rapidly rotating than $\mathrm{K}$ giants.

\section{b. N-type carbon stars}

Molecular spectra so dominate the spectra of the Ntype stars that abundance analyses have been seriously hampered. Even with the advent of spectrum synthesis as an analytical tool, progress has been slow. In part, this is due to the fact that the many molecular bands are much more richly represented in the stellar spectra than in laboratory sources; unidentified lines are commonly a nuisance. The principal results can best be set out in the format used above for the R stars.

(1) Metallicity. Kinematics of N stars suggests solarlike metallicities. Spectroscopic estimates are few, uncertain, and based on few lines. Lambert et al. (1986) found $[\mathrm{Fe} / \mathrm{H}]=0$ to within about \pm 0.3 dex from a handful of infrared lines of $\mathrm{Na}, \mathrm{Ca}$, and $\mathrm{Fe}$ in a sample of 30 bright carbon stars.

(2) Lithium. Lithium abundances of the majority of $\mathrm{N}$ stars appear to be at the same level as in normal M giants (Denn et al., 1991; Abia et al., 1993) which implies that transformation of $\mathrm{M}$ giants to $\mathrm{N}$ stars by operation of the third dredge up has not substantially altered the lithium abundance. This result seems compatible with calculations of the third dredge up. A few stars are so remarkably enriched in lithium that production of lithium must have occurred. These stars are the J-type stars having a low ${ }^{12} \mathrm{C} /{ }^{13} \mathrm{C}$ ratio and little or no enhancement of $s$-process elements (Dominy, 1985). Abia and Isern (1996) suggest that lithium increases with decreasing ${ }^{12} \mathrm{C} /{ }^{13} \mathrm{C}$ ratio but this may not reflect a physical relation as the $\mathrm{N}$ and $\mathrm{N}(\mathrm{J})$ stars appear so very different in $s$-process abundances. One is tempted to identify the $\mathrm{R}$ stars as progenitors of the $\mathrm{N}(\mathrm{J})$ stars. Then, the $\mathrm{N}(\mathrm{J})$ stars must manufacture lithium or lithium-rich $\mathrm{R}$ stars have gone undetected. Unusual constraints are placed on lithium manufacture in this case: no prior or attendant $s$ production or reduction of the $\mathrm{C} / \mathrm{O}$ ratio.

(3) $C, N$, and $O$. Elemental abundance were given by Lambert et al. (1986) from infrared spectra that contained $\mathrm{CO}, \mathrm{C}_{2}, \mathrm{CN}$, and other molecular lines. Three results deserve brief mention. First, the oxygen abundances $[\mathrm{O} / \mathrm{H}]$ were on average slightly subsolar $([\mathrm{O} / \mathrm{H}]$ $\approx-0.2$ ). The oxygen abundances were in good agreement with those found for $\mathrm{M}$ giants (progenitors of $\mathrm{N}$ stars?) and for planetary nebulae (descendants of $\mathrm{N}$ stars?). Second, the $\mathrm{C} / \mathrm{O}$ ratios show the carbon enrichment to be relatively small: the most carbon-rich star had $\mathrm{C} / \mathrm{O} \sim 1.6$ and about half of the sample had $\mathrm{C} / \mathrm{O}<1.1$.
These values do show that substantial amounts of carbon have been added to convert an $\mathrm{M}$ giant $(\mathrm{C} / \mathrm{O} \sim 0.3)$ to a $\mathrm{N}$ star but there are planetary nebulae that are significantly more C rich (Zuckerman and Aller, 1986). Third, the nitrogen abundances of the $\mathrm{N}$ stars were less than those derived for the $\mathrm{M}$ (and $\mathrm{K}$ ) giants by about 0.6 dex. M giants presumably may evolve to $\mathrm{N}$ stars, but it is difficult to imagine that nitrogen can be destroyed by an AGB star. (Many $\mathrm{M}$ giants may lose their envelope as O-rich AGB stars and not evolve to $\mathrm{N}$ stars.) The mean nitrogen abundance of the $\mathrm{N}$ stars $([\mathrm{N} / \mathrm{H}]=-0.3 \pm 0.2)$ is less than that from C-rich planetary nebulae for which $[\mathrm{N} / \mathrm{H}]=0.1 \pm 0.3$ from Zuckerman and Aller (1986). Lambert et al. (1986) stress that their $\mathrm{N}$ abundance determinations are particularly susceptible to systematic errors.

Isotopic abundances of carbon were derived in three ways using infrared lines of two molecules; $\mathrm{CN}$ and $\mathrm{CO}$. The three results were in good agreement. The range $30<{ }^{12} \mathrm{C} /{ }^{13} \mathrm{C}<70$ encompassed the larger part of the sample. The $\mathrm{N}(\mathrm{J})$ stars had, of course, lower values close to the equilibrium value for the $\mathrm{CN}$ cycle. Lambert et al. (1986) argued that the ${ }^{12} \mathrm{C} /{ }^{13} \mathrm{C}$ ratios of $\mathrm{N}$ stars resulted from the addition of ${ }^{12} \mathrm{C}$ but not ${ }^{13} \mathrm{C}$ to the atmosphere of a $\mathrm{M}$ giant with its lower carbon abundance and lower ${ }^{12} \mathrm{C} /{ }^{13} \mathrm{C}$ ratio. Recently, Ohnaka and Tsuji (1996) have measured ${ }^{12} \mathrm{C} /{ }^{13} \mathrm{C}$ ratios from near-infrared $\mathrm{CN}$ bands obtaining lower ratios than those given by Lambert et al. (1986). Although it does not seem critical to evolutionary interpretation of $\mathrm{N}$ stars, this discrepancy needs to be examined.

A general point of great importance may be inserted at this juncture. Two different observational approaches now provide information on the isotopic carbon (and other) ratios. First, observations of molecular millimeter and radio line emission from circumstellar shells provide independent data. Presently, the reliable data refer to AGB stars whose photospheric spectra have not been analyzed for the same ratios. Kahane et al. (1992) provide the ${ }^{12} \mathrm{C} /{ }^{13} \mathrm{C}$ ratio for shells of five carbon stars. The results $\left(>65,32_{-7}^{+10},>30,44 \pm 3\right.$, and $\left.31_{-5}^{+6}\right)$ might be viewed as supporting the results given by Lambert et al. (1986). Second, the isolation of $\mathrm{SiC}$ grains from carbonaceous chondrites also provides new data on carbon stars, which are the most likely source of these surviving circumstellar grains. The distribution of ${ }^{12} \mathrm{C} /{ }^{13} \mathrm{C}$ ratios for $\mathrm{SiC}$ grains mimics well the stellar distribution found by Lambert et al. (1986) ( see also Anders and Zinner, 1993).

Oxygen isotopic abundances in carbon stars were estimated by Harris and Lambert (1987) following earlier determinations of the abundances for oxygen-rich stars on and off the AGB (Harris and Lambert, 1984, 1987; Harris et al., 1985, 1988). Kahane et al. (1992) provide the ratio ${ }^{16} \mathrm{O} /{ }^{18} \mathrm{O}$ and ${ }^{16} \mathrm{O} /{ }^{17} \mathrm{O}$ for circumstellar shells of five carbon stars. Analyses of oxide grains from primitive meteorites (Nittler et al., 1994) has also provided these ratios which pertain presumably to circumstellar shells of oxygen-rich (now deceased) AGB stars. Theoretical work on interpreting the measured oxygen isoto- 
pic ratios has been quite extensive. Forestini and Charbonnel (1997) indicate that their models of intermediate mass AGB stars can explain the ratios except where the ${ }^{16} \mathrm{O} /{ }^{17} \mathrm{O}$ ratio is high; for example, they remark that the measured isotopic carbon, nitrogen, and oxygen ratios for nearby dust-shrouded carbon star IRC+10216 (CW Leo) are consistent with their predictions for an AGB star of mass 4 to $5 M_{\odot}$ and solar metallicity except that the observed ${ }^{16} \mathrm{O} /{ }^{17} \mathrm{O}$ ratio is not well explained. Wasserburg et al. (1995) invoke deep mixing in red ( $\mathrm{H}$-shell burning) giants to explain the oxygen isotopic abundance in giants (before and on the the AGB) and in the meteoritic oxide grains.

(4) $s$ process. The $\mathrm{N}$ stars are $s$-process enriched but the $\mathrm{N}(\mathrm{J})$ are not. Quantitative assessments of the $s$-process abundances in $\mathrm{N}$ stars come from a pioneering study by Utsumi $(1967,1970,1985)$ based on photographic spectra in "windows" at 4750-4900 $\AA$ and 4400$4500 \AA$ between $\mathrm{C}_{2}$ bands. Busso et al. compare the abundances of the $s$ elements with those of Ba II and other $s$-process enriched stars and with predictions for AGB stars experiencing the third dredge up. In short, the observations for the carbon stars are consistent with predictions as to neutron exposure and mixing fraction (see V. V. Smith, Sec. XI, for further discussion). Moreover, the observations of $\mathrm{N}$ stars are consistent with the hypothesis that $\mathrm{Ba}$ II and other stars result from transfer of mass to the present star from an AGB $s$-process enriched star; the $s$-process enrichment of Ba II stars is less than that of the carbon stars (i.e., diluted following mass transfer) but of a similar pattern as regards light to heavy $s$ elements. The large enhancement of $s$-process species in the $\mathrm{CH}$ stars is discussed in Sec. XI.

A clear signature of active or recent $s$ processing is the presence of technetium, as noted by $\mathrm{B}^{2} \mathrm{FH}$ for carbon stars (Merrill, 1956). Little-Marenin's (1989) review of technetium in stars reports observations of $15 \mathrm{~N}$ stars of which 12 showed this unstable element. One N(J) star did not show technetium. The absence of technetium from the $\mathrm{N}(\mathrm{J})$ is expected as these stars are not $s$-process enhanced. The absence of the technetium from $3 \mathrm{~N}$ stars does not necessarily imply the stars are not active AGB stars. Detection of the Tc I lines in spectra of cool carbon stars is difficult. There is also the possibility that continuous decay of technetium may reduce the abundance below detectable limits.

No review of carbon stars, especially one discussing the convergence of observation and theory, could be considered complete without mention of the carbon stars in the Magellanic Clouds. Since the Clouds are at a known distance, stars may be assigned absolute luminosities. In contrast, distances to carbon stars in our Galaxy and, therefore, luminosities are quite uncertain. The luminosity distribution function for carbon stars in the Clouds showed an absence of the predicted very luminous carbon stars and peaked at a luminosity too low to correspond to the intermediate-mass AGB stars for which the third dredge up had been predicted to operate. A seminal discussion of the luminosity function is that by Iben (1981). Two points should be stressed. First, the marked absence of luminous carbon AGB stars is now attributed primarily to two effects: (i) severe mass loss that may terminate AGB evolution before high luminosities are attained, and (ii) the onset of hot bottom burning that reconverts the carbon-rich to an oxygenrich AGB star. Second, as emphasised by Iben (1981), the "low" mean luminosity of carbon stars implies that low mass AGB stars also experience the third dredge up, an observation not predicted by then extant calculations. Iben wrote "a significant fraction of the carbon that is freshly synthesised in an AGB star during thermal pulses is brought to the surface of every AGB star in the Clouds, whatever the mass of the underlying carbonoxygen core." Understanding mass loss and the third dredge up in low mass AGB stars remain the key theoretical problems in AGB evolution.

Luminous AGB stars do exist in the Clouds, as emphasised by Wood et al. (1983). These are S stars (i.e., enriched in $s$ elements) and all are lithium rich (Smith and Lambert, 1989, 1990; Plez et al., 1993; Smith et al., 1995). The luminosities of the lithium-rich stars and the lithium abundances are consistent with the predictions for hot bottom burning (Sackmann and Boothroyd, 1992). In this phase, a very luminous AGB star supports hydrogen burning at the base of the convective envelope where previously the hydrogen burning shell was below the base of the convective envelope. Hot bottom burning converts carbon to nitrogen and reduces the $\mathrm{C} / \mathrm{O}$ ratio below unity. Lithium is produced by the chain ${ }^{3} \mathrm{He}\left({ }^{4} \mathrm{He}, \gamma\right){ }^{7} \mathrm{Be}(e, \nu){ }^{7} \mathrm{Li}$ (Cameron and Fowler, 1971) where the ${ }^{3} \mathrm{He}$ was produced in the main sequence star. This cannot account for all the lithium-rich stars in the Clouds as some have lower luminosities than predicted for hot bottom burning. One is reminded of the lithiumrich galactic carbon stars.

\section{c. Barium and related stars}

To $\mathrm{B}^{2} \mathrm{FH}$ the $\mathrm{Ba}$ II stars were a laboratory in which to test predicted abundance patterns for the $s$ process. Certainly, then and now, the spectra of Ba II K giants are more tractable than the spectra of the $\mathrm{N}$ stars with similar $s$-process enrichments. Early attempts to account for the $\mathrm{Ba}$ II and $\mathrm{CH}$ giants, which are metal-poor C-rich stars, focused on the helium core flash that occurs in low $\left(M<2.3 M_{\odot}\right)$ mass stars when helium is ignited in the electron degenerate core. This explosive event was seen by some observers as the driver for the nucleosynthesis and mixing needed to transform a star to a barium star. An early call on the helium core flash as the deus ex machina was made by Warner (1965) with, perhaps, the last call by Tomkin and Lambert (1983). The luminosities of the barium stars seemed compatible with the idea too; the mean luminosity appeared to be that of a clump or horizontal branch (i.e., a post-flash helium core burning star).

Discovery of the CH subgiants by Bond (1974) showed that stars with compositions of the Ba II giants existed at luminosities too faint for helium core burning giants. The $\mathrm{Ba}$ II phenomenon appeared to extend to the main sequence. A speculation was advanced that the 
helium core flash led to such extensive mixing that, with hydrogen replenished in the core, the postflash star assumed again the structure (and luminosity) of a main sequence star (see also Luck and Bond, 1982).

All attachments to the helium core flash by observers were shattered with the delightful discovery that all $\mathrm{Ba}$ II and CH stars are long period spectroscopic binaries. This result was first reported by McClure et al. (1980)reviews by McClure $(1985,1989)$ should be read! This discovery was a reminder that binary stars must be considered to obtain a complete picture of stellar evolution and a full understanding of peculiar stars. The discovery led to the idea that mass transfer between components of a binary star may create a low luminosity star with a composition similar to that of an AGB star. The helium core flash was cast aside by observers.

A prediction of the mass transfer hypothesis is that main sequence barium stars should exist. Happily, examples have been found by Tomkin et al. (1989), Edvardsson et al. (1993), and North et al. (1994).

For a discussion of the compositions of barium stars of all kinds, the reader is referred to Lambert $(1985,1988)$, Barbuy et al. (1992), Vanture (1992a, 1992b), with V. V. Smith, Sec. XI of this review, providing remarks on the $s$ process. A comparison of the compositions of barium stars with those of active AGB stars including the Ntype carbon stars shows the great similarities required under the mass transfer hypothesis. Mass transfer is most probably effected by accretion of gas and dust from the AGB star's wind (Boffin and Jorissen, 1988).

The main sequence barium stars just mentioned are all stars with spectral types of F and G. Cooler stars will show strong molecular bands and may look at low spectral resolution like N-type (giant) stars. The first such case was G77-61 with a classical carbon star spectrum but a high proper motion suggesting a main sequence luminosity. It is a spectroscopic binary and mass transfer is a likely explanation for it (Dearborn et al., 1986). Additional examples have now been found (Green et al., 1991; Green et al., 1992; Warren et al., 1993). An abundance analysis of G77-61 (Gass et al., 1988) indicates that the dwarf carbon stars may not be simply cool close relatives of the barium $F$ and $G$ main sequence stars.

Gass et al. (1988) conclude that G77-61 has low metallicity: $[\mathrm{Fe} / \mathrm{H}] \approx-5.6$ but a normal hydrogen to helium ratio. Relative to iron, carbon is substantially enriched $([\mathrm{C} / \mathrm{H}] \approx-1)$. No $s$-process element was measured. Gass et al. suppose the star was initially a very metal-poor star with a composition reflecting contamination of primordial gas by debris from a very massive star. Perhaps, there is a simpler alternative to consider: gas but not dust was accreted as part of the mass transfer process that led to the dwarf carbon star. Gas in circumstellar winds off AGB stars is highly depleted in those elements that form or adhere to grains. Iron and other elements will be underabundant in the gas. If gas and not dust is accreted by the companion in large amounts, the resulting star could be metal poor. Until other dwarf carbon stars are analyzed, scepticism might be maintained about their origin and their link to other main sequence barium stars. This is an important area for future spectroscopic work because de Kool and Green (1995) suggest that "dwarf $\mathrm{C}$ stars may be the numerically dominant type of carbon star in the Galaxy."

\section{Epilogue}

In 1957, $\mathrm{B}^{2} \mathrm{FH}$ 's bold discussions of nuclear astrophysics and element synthesis were many years ahead of an understanding of stellar evolution, which has come in two main areas. First, enormous progress has been made in observational abundance studies and theoretical modeling of single AGB stars. In broad terms, observed AGB stars and the theoretical models of these stars have converged. It may be said that the N-type carbon stars are understood. Models also show how hot bottom burning converts the carbon star back to an S star. Second, the appreciation that $\mathrm{Ba}$ II and related stars are binaries introduced the idea that mass transfer from an AGB star suffices to account for these stars. Addition of an innocent companion in a wide orbit provides the opportunity to transform that companion to a barium star. Classical Ba II stars, CH giants, and chemically similar stars of lower and higher luminosity surely result from accretion of mass shed by an oxygen or carbon rich AGB star. These expressions of convergence between observation and theory should not hide vital topics requiring additional scrutiny. On the theoretical side, present treatments of the third dredge up and of mass loss by a wind need to be replaced by accurate $a b$ initio theories. On the observational side, quantitative spectroscopy of the cool carbon stars needs to be refined, and the advent of large telescopes in the south should be exploited through high-resolution spectroscopy of AGB stars in the Magellanic Clouds.

Classes of stars for which observation and theory have yet to converge particularly deserve further examination. In this review, we have mentioned just some of these recalcitrant classes. We recall the $\mathrm{R}$ [and $\mathrm{N}(\mathrm{J})$ ] stars: do they result from an aberrant heliuim core flash or coalescence of stars in a binary system? The dwarf C stars deserve a mention, particularly if G77-61 with $[\mathrm{Fe} / \mathrm{H}] \approx-5.6$ is seen to be typical. Observers should take note of this tip of the dwarf carbon star iceberg. Other classes of carbon stars were not mentioned; for example, the $\mathrm{R} \mathrm{CrB}$ and related hydrogen deficient stars, stars that may be examples of "born again" supergiants experiencing a final helium shell flash (FG Sge?), and carbon stars with circumstellar shells of silicate grains. Perhaps, at the next major anniversary of the classic tale "Synthesis of the Elements in Stars," the convergence of observation and theory will have been extended to all carbon stars. Perhaps!

\section{CONCLUSIONS}

Forty years after the publication of $\mathrm{B}^{2} \mathrm{FH}$, we find that new discoveries and developments in nucleosynthesis and stellar evolution (astronomical observations, laboratory measurements, and model calculations) have 
in large measure borne out and expanded the insights of the original authors. For example, the development of mass spectroscopy techniques have made possible the isotopic analysis of individual meteorite grains, while the expansion of the observational spectrum to include gamma rays and neutrinos have made possible the study of such diverse processes as the distribution of ${ }^{26} \mathrm{Al}$ in our galaxy, neutrinos from the center of our sun, and the neutrinos and ${ }^{56} \mathrm{Fe}$ gamma rays from the collapse and subsequent decay of SN-1987a in the Large Magellenic Cloud, some 55 kiloparsecs away. At the same time a number of important open questions still remain, relating to areas ranging from the resolution of the solar neutrino problem to the determination of the important ${ }^{14} \mathrm{~N}(p, \gamma)$ and ${ }^{12} \mathrm{C}(\alpha, \gamma)$ nuclear reaction rates both of which are still uncertain to approximately a factor of 2 due to questions associated with the role of subthreshold resonances in the low-energy extrapolation of laboratory measurements.

The remaining uncertainties on the astronomy side are substantial and, in some cases, difficult to overcome. While the opacity calculations for stellar structure calculations have been greatly improved by the inclusion of bound-bound transitions of iron and some other species (Rogers and Iglesias, 1992) the problems of convection theory remain. In the deep interior of stars more massive than about $1.5 M_{\odot}$ the core in which the $\mathrm{CNO}$ cycle and later helium burning is taking place is convective. A problem arises with the extent to which convection penetrates beyond the nuclear reaction region thereby bringing in fresh fuel and extending the lifetime of the star in that particular state. In stars cooler than about $6000 \mathrm{~K}$ at their surfaces an outer convection zone develops that homogenizes the layers in which it is active. The depth to which this convection zone penetrates is uncertain and the temperature gradient in the superadiabatic region is difficult to calculate.

The analysis of the chemical composition of stars has improved vastly during the past 40 years due to the replacement of photographic plates by digital detectors and the construction of new large telescopes such as Keck I and II. Very soon we should see high resolution spectra of stars in nearby systems at distances of 100 kiloparsecs. The analyses of stellar spectra have been greatly improved by the routine use of model stellar atmospheres, but the models have their limitations. The most important of these is the difficult problem of the boundary temperature for a star of given effective temperature, since the strengths of an absorption line is very sensitive to the temperature gradient, not just the stellar effective temperature. Two stars of the same effective temperature may have different degrees of chromospheric heating and hence different backwarming of the outer layers of the atmosphere (Kurucz, 1996).

Finally, we must mention the ever-improving but never good enough atomic data, especially the transition probabilities, that are vital to the analysis of atomic absorption lines. The need is especially great for the rare earth and heavier species produced by the $s, r$, and $p$ processes. Similarly, for both oxide and carbon mol- ecules, we need transition probabilities accurate to better than 0.1 dex and dissociation potentials that are accurate to better than $0.1 \mathrm{eV}$ to derive abundances of various elements in cool stars.

Finally, we call attention to the complexity of the events during a supernova explosion which is responsible for the production of almost all the species from $\mathrm{Si}$ to $\mathrm{Zn}$ as well as the $r$-process elements. Both the great progress and remaining uncertainties are fully discussed in the monograph of Arnett (1996).

\section{ACKNOWLEDGMENTS}

This paper originated in the invitation by Michael Wiescher to one of us (G.W.) to present the introductory talk at the "Nuclei in the Cosmos III" conference at Notre Dame University in June, 1996. The research of the many authors has been supported by the National Science Foundation, Department of Energy, The National Aeronautics and Space Administration, the Robert Welch Foundation, and the Kennilworth Fund of the New York Community Trust. In addition, we want to express our appreciation to our home institutions for providing us with the opportunity to have conducted research in nuclear astrophysics, stellar evolution, and observational astronomy over the (in some cases, many) years. Finally, each individual author acknowledges assistance, comments, and criticism from a number of colleagues, the total list of whom is too long to include here. Special acknowledgment is due Ms. Karen Fisher who assembled the various manuscripts and translated a variety of Tex forms into the single form of this journal.

\section{REFERENCES}

Abbot, D. C., and P. S. Conti, 1987, Annu. Rev. Astron. Astrophys. 25, 113.

Abia, C., H. M. Boffin, J. Isern, and R. Rebolo, 1993, Astron. Astrophys. 272, 455.

Abia, C., and J. Isern, 1996, Astrophys. J. 460, 443.

Ajzenberg-Selove, F., 1990, Nucl. Phys. A 506, 1.

Alexander, T. K., G. C. Ball, W. N. Lennard, H. Geissel, and H. B. Mak, 1984, Nucl. Phys. A 427, 526.

Allen, C. W., 1973, Astrophysical Quantities (Athlone, London).

Almqvist, E., D. A. Bromley, and J. A. Kuehner, 1960, Phys. Rev. Lett. 4, 515.

Almqvist, E., D. A. Bromlev, and J. A. Kvehner, 1963, Phys. Rev. 130, 1140.

Alpar, M. A., A. F. Cheng, M. A. Ruderman, and J. Shalam, 1982, Nature (London) 316, 681.

Alpar, M. A., and J. Shalam, 1985, Nature (London) 317, 681. Alvarez, L., 1949, University of California Radiation Laboratory Report No. UCRL-328 (unpublished).

Anders, E. H., and N. Grevesse, 1989, Geochim. Cosmochim. Acta 53, 197.

Anders, E. H., and E. Zinner, 1993, Meteoritics 28, 490.

Anderson, M. R., S. R. Kennett, L. W. Mitchell, and D. G. Sargood, 1980, Nucl. Phys. A 349, 154.

Anderson, M. R., L. W. Mitchell, S. E. Sevior, S. R. Kennett, and D. G. Sargood, 1982, Nucl. Phys. A 373, 326. 
Arnett, D., 1995, Annu. Rev. Astron. Astrophys. 33, 115.

Arnett, D., 1996, Supernovae and Nucleosynthesis (Princeton University, Princeton, NJ).

Arnett, D., J. N. Bahcall, R. P. Kirshner, and S. E. Woosley, 1989, Annu. Rev. Astron. Astrophys. 27, 629.

Arnett, D., J. W. Truran, and S. E. Woosley, 1971, Astrophys. J. 165, 87.

Arnould, M., 1976, Astron. Astrophys. 46, 117.

Arnould, M., N. Mowlavi, and A. E. Champagne, 1995, Proceedings of the 32nd Liége International Astrophysics Coll. Arpesella, C., et al., 1996, Phys. Lett. B 389, 452.

Assenbaum, H. J., K. Langanke, and C. Rolfs, 1987, Z. Phys. A 327, 461.

Audouze, J., and J. W. Truran, 1975, Astrophys. J. 202, 204.

Aufderheide, M. B., E. Baron, F.-K. Thielemann, 1991, Astrophys. J. 370, 630 .

Aufderheide, M. B., I. Fushiki, S. E. Woosley, and D. H. Hartmann, 1994, Astrophys. J. Suppl. 91, 398.

Azuma, R. E., R. E. L. Buchmann, F. C. Barker, C. A. Barnes, J. M. D'Auria, M. Dombsky, U. Giesen, K. P. Jackson, J. D. King, R. G. Korteling, P. McNeely, J. Powell, G. Roy, J. Vincent, T. R. Wang, S. S. M. Wong, and P. R. Wrean, 1994, Phys. Rev. C 50, 1194.

Baade, W., and R. Minkowski, 1957, cited in $\mathrm{B}^{2} \mathrm{FH}$ as a private communication.

Bahcall, J. N., 1961, Phys. Rev. 124, 495.

Bahcall, J. N., 1964a, Astrophys. J. 139, 318.

Bahcall, J. N., 1964b, Phys. Rev. Lett. 12, 300.

Bahcall, J. N., 1989, Neutrino Astrophysics (Cambridge University Press, Cambridge, England).

Bahcall, J. N., 1994, Phys. Lett. B 338, 276.

Bahcall, J. N., and R. M. May, 1969, Astrophys. J. 155, 501.

Bahcall, J. N., and M. H. Pinsonneault, 1992, Rev. Mod. Phys. 64, 885.

Bahcall, J. N., M. H. Pinsonneault, and X. Wasserburg, 1995, Rev. Mod. Phys. 69, 781.

Balachandran, S., 1990, Astrophys. J. 354, 310.

Balsar, D. S., T. M. Bania, R. T. Rood, and T. L. Wilson, 1997, Astrophys. J. 483, 320.

Bao, Z. Y., and F. Käppeler, 1987, At. Data Nucl. Data Tables 36, 411.

Baraffe, I., M. F. El Eid, and N. Prantzos, 1992, Astron. Astrophys. 258, 357.

Barbuy, B., A. Jorissen, S. C. F. Rossi, and M. Arnould, 1992, Astron. Astrophys. 262, 216.

Barker, F. C., 1971, Aust. J. Phys. 24, 777.

Barker, F. C., 1987, Aust. J. Phys. 40, 25.

Barker, F. C., and T. Kajino, 1991, Aust. J. Phys. 44, 369.

Barnes, C. A., S. Trentalange, and S.-C. Wu, 1985, in Treatise on Heavy Ion Science, Vol. 6, edited by D. A. Bromley (Plenum, New York).

Barnett, R. M., et al., 1996, Phys. Rev. D 54, 1.

Basri, G., E. L. Martin, and C. Bertout, 1991, Astron. Astrophys. 252, 625.

Bazan, C., and W. D. Arnett, 1994a, Astrophys. J. 433, L41.

Bazan, C., and W. D. Arnett, 1994b, Astrophys. J. Lett. 41, 3. Beaudet, G., V. Petrosian, and E. E. Salpeter, 1967, Astrophys. J. 150, 979.

Becker, H. W., H. Ebbing, W. H. Shulte, S. Wüstenbecker, M. Berheide, M. Buschmann, C. Rolfs, G. E. Mitchell, and J. S. Schweizer, 1992, Z. Phys. A 343, 361.

Becker, H. W., K. U. Kettner, C. Rolfs, and H. P. Trautvetter, 1981, Z. Phys. A 303, 305.
Becker, S. A., 1981, in Physical Processes in Red Giants, edited by I. Iben and A. Renzini (Reidel, Dordrecht), p. 141.

Becker, S. A., and I. Iben, Jr., 1980, Astrophys. J. 232, 831.

Beer, H., F. Corvi, and P. Mutti, 1997, Astrophys. J. 474, 843.

Beer, H., and R. L. Macklin, 1989, Astrophys. J. 339, 962.

Beer, H., G. Walter, and R. Macklin, 1985, in Capture GammaRay Spectroscopy and Related Topics, edited by S. Raman (AIP, New York), p. 778.

Beers, T. C., G. W. Preston, and S. A. Shectman, 1985, Astron. J. 90, 2089.

Beers, T. C., G. W. Preston, and S. A. Schetman, 1992, Astron. J. 103, 1987.

Benz, W., R. L. Bowers, A. G. W. Cameron, W. Press, 1990, Astrophys. J. 348, 647.

Berg, H. L., W. Hietze, C. Rolfs, and H. Winkler, 1977, Nucl. Phys. A 276, 168.

Bethe, H. A., 1939, Phys. Rev. 55, 434.

Bethe, H. A., and C. L. Critchfield, 1938, Phys. Rev. 54, 248.

Bhattacharya, D., and E. P. J. van den Heuvel, 1991, Phys.

Rep. 203, 1.

Bidelman, W. P., 1953, Astrophys. J. 117, 25.

Bidelman, W. P., 1956, Vistas Astron. 2, 1428.

Bidelman, W. P., and P. C. Keenan, 1951, Astrophys. J. 114, 473.

Biehle, G., 1991, Astrophys. J. 380, 167.

Black, D. C., 1972, Geochim. Cosmochim. Acta 36, 377.

Blackmon, J. C., A. E. Champagne, J. K. Dickens, J. A. Harvey, M. A. Hofstee, S. Kopecky, D. C. Larson, D. C. Powell, S. Raman, and M. S. Smith, 1996, Phys. Rev. C 54, 383.

Blackmon, J. C., A. E. Champagne, M. A. Hofstee, M. S. Smith, R. G. Downing, and G. P. Lamaze, 1995, Phys. Rev. Lett. 74, 2642.

Blank, B., S. Andriamonje, S. Czajkowski, F. Davi, R. Del Moral, J. P. Dufour, A. Fleury, A. Musquére, M. S. Pravikoff, R. Grzywacz, Z. Janas, M. Pfützner, A. Grewe, A. Heinz, A. Junghans, M. Lewitowicz, J.-E. Sauvestre, and C. Donzaud, 1995, Phys. Rev. Lett. 74, 4611.

Blöcker, T., F. Herwig, D. Schönberner, and M. El Eid, 1997, The Carbon Star Phenomenon, ASP Conf. Ser., in press.

Bodansky, D., D. D. Clayton, and W. A. Fowler, 1968, Astrophys. J. Suppl. 16, 299.

Boesgaard, A. M., 1970, Astrophys. J. 161, 1003.

Boesgaard, A. M., 1976, Astrophys. J. 210, 466.

Boesgaard, A. M., 1991, Astrophys. J. 370, L95.

Boesgaard, A. M., 1996, ASP Conf. Series, edited by H. L. Morrison and A. Sarajedini (ASP San Francisco), Vol. 92, p. 327.

Boesgaard, A. M., C. P. Deliyannis, J. R. King, and A. Stephens, 1997, Astrophys. J., in press.

Boesgaard, A. M., and W. D. Heacox, 1978, Astrophys. J. 226, 888.

Boesgaard, A. M., and J. R. King, 1993, Astron. J. 106, 2309.

Boesgaard, A. M., F. Praderie, D. S. Leckrone, R. Faraggiana, and M. Hack, 1974, Astrophys. J. 194, L143.

Boesgaard, A. M., and G. Steigman, 1985, Annu. Rev. Astron. Astrophys. 23, 319.

Boesgaard, A. M., and M. J. Tripicco, 1986, Astrophys. J. 303, L42.

Boffin, H. M. J., and A. Jorissen, 1988, Astron. Astrophys. 205, 155.

Bond, H. E., 1974, Astrophys. J. 194, 95. 
Boothroyd, A. I., and I.-J. Sackmann, 1997, Astrophys. J. (submitted).

Bosch, F., T. Faestermann, J. Friese, F. Heine, P. Kienle, E. Wefers, K. Zeitelhack, K. Beckert, B. Franzke, O. Klepper, C. Kozhuharov, G. Menzel, R. Moshammer, F. Nolden, H. Reich, B. Schlitt, M. Steck, T. Stöhlker, T. Winkler, and K. Takahashi, 1996, Phys. Rev. Lett. 77, 5190.

Bowen, G. H., 1988, Astrophys. J. 329, 299.

Bowen, G. H., and L. A. Willson, 1991, Astrophys. J. 375, L53.

Boyd, R. N., 1994, Int. J. Mod. Phys. Suppl. E3, 249.

Bressan, A., F. Fagotto, G. Bertelli, and C. Chiosi, 1992, Astron. Astrophys. Suppl. Ser. 100, 647.

Briley, M. M., R. A. Bell, J. E. Hesser, and G. H. Smith, 1994, Can. J. Phys. 72, 772.

Briley, M. M., V. V. Smith, N. B. Suntzeff, D. L. Lambert, R. A. Bell, and J. E. Hesser, 1996, Nature (London) 383, 604.

Brown, J. A., V. V. Smith, D. L. Lambert, E. Dutchover, Jr., K. H. Hinkle, and H. R. Johnson, 1990, Astron. J. 99, 1930.

Brown, J. A., G. Wallerstein, and J. B. Oke, 1991, Astron. J. 101, 1693.

Buchmann, L., R. E. Azuma, C. A. Barnes, J. M. D'Auria, M. Dombsky, U. Giesen, K. P. Jackson, J. D. King, R. G. Korteling, P. McNeely, J. Powell, G. Roy, J. Vincent, T. R. Wang, S. S. M. Wong, and P. R. Wrean, 1993, Phys. Rev. Lett. 70, 726.

Buchmann, L., R. E. Azuma, C. A. Barnes, J. Humblet, and K. Langanke, 1996, Phys. Rev. C 54, 393.

Buchmann, L., H. W. Becker, K. U. Kettner, W. E. Kieser, P. Schmalbrock, and C. Rolfs, 1980, Z. Phys. A 296, 273.

Buchmann, L., M. Hilgemeier, A. Krauss, A. Redder, C. Rolfs, and H. P. Trautvetter, 1984, Nucl. Phys. A 415, 93.

Burbidge, E. M., G. R. Burbidge, W. A. Fowler, and F. Hoyle, 1957, Rev. Mod. Phys. 29, 547.

Burbidge, G. R., and E. M. Burbidge, 1957, Astrophys. J. 126, 357.

Burrows, A., and J. Hayes, 1996, Phys. Rev. Lett. 76, 352.

Burrows, A., J. Hayes, and B. A. Fryxell, 1995, Astrophys. J. 450, 830.

Burrows, A., and J. M. Lattimer, 1986, Astrophys. J. 307, 178. Burrows, A., and J. M. Lattimer, 1987, Astrophys. J. 318, L63. Busso, M., R. Gallino, D. L. Lambert, C. M. Raiteri, and V. V. Smith, 1992, Astrophys. J. 399, 218.

Busso, M., D. L. Lambert, L. Beglio, R. Gallino, C. M. Raiteri, and V. V. Smith, 1995, Astrophys. J. 446, 775.

Butcher, H. R., 1987, Nature (London) 328, 127.

Cameron, A. G. W., 1955, Astrophys. J. 121, 144.

Cameron, A. G. W., 1957, Chalk River Report CRL-41.

Cameron, A. G. W., 1961, Astron. J. 65, 485.

Cameron, A. G. W., and W. A. Fowler, 1971, Astrophys. J. 164, 111.

Cannon, R., 1993, Mon. Not. R. Astron. Soc. 263, 817.

Canuto, V., 1970, Astrophys. J. 159, 641.

Carlsson, M., R. J. Rutten, J. H. M. J. Bruls, and N. G. Shchukina, 1994, Astron. Astrophys. 288, 860.

Cassinelli, J. P., 1979, Annu. Rev. Astron. Astrophys. 11, 275. Caughlan, G. R., and W. A. Fowler, 1962, Astrophys. J. 136, 453.

Caughlan, G. R., and W. A. Fowler, 1988, At. Data Nucl. Data Tables 40, 284.

Cavallo, R. M., A. V. Sweigart, and R. A. Bell, 1996, Astrophys. J. 464, L79.

Cavallo, R. M., A. V. Sweigart, and R. A. Bell, 1997, Astrophys. J. (in press).
Chaboyer, B., and P. Demarque, 1994, Astrophys. J. 433, 510. Chaboyer, B., P. Demarque, P. J. Kernan, and L. M. Krauss, 1996, Science 271, 957.

Chamberlain, J. W., and L. H. Aller, 1951, Astrophys. J. 114, 52.

Champagne, A. E., B. A. Brown, and R. Sherr, 1993, Nucl. Phys. A 556, 123.

Champagne, A. E., C. H. Cella, R. T. Kouzes, M. M. Lowry, P. V. Magnus, M. S. Smith, and Z. Q. Mao, 1988, Nucl. Phys. A 487, 433.

Champagne, A. E., A. J. Howard, and P. D. Parker, 1983, Nucl. Phys. A 402, 159.

Champagne, A. E., A. J. Howard, M. S. Smith, P. V. Magnus, and P. D. Parker, 1989, Nucl. Phys. A 505, 384.

Champagne, A. E., P. V. Magnus, M. S. Smith, and A. J. Howard, 1990, Nucl. Phys. A 512, 317.

Champagne, A. E., A. B. McDonald, T. F. Wang, A. J. Howard, P. V. Magnus, and P. D. Parker, 1986a, Nucl. Phys. A 451, 498.

Champagne, A. E., M. L. Pitt, P. H. Zhang, L. L. Lee, Jr., and M. J. LeVine, 1986b, Nucl. Phys. A 459, 239.

Charbonnel, C., 1995, Astrophys. J. 453, L41.

Charbonnel, C., G. Meynet, A. Maeder, G. Schaller, and D. Schaerer, 1993, Astron. Astrophys. Suppl. Ser. 101, 415.

Chen, K., and M. Ruderman, 1993, Astrophys. J. 408, 179.

Chevalier, R. A., 1996, Astrophys. J. 459, 322.

Chiosi, C., G. Bertelli, and A. Bressan, 1992, Annu. Rev. Astron. Astrophys. 30, 235.

Chiosi, C., and A. Maeder, 1986, Annu. Rev. Astron. Astrophys. 24, 329.

Chmielewski, Y., E. Müller, and J. W. Brault, 1975, Astron. Astrophys. 42, 37.

Clayton, D. D., 1968, Principles of Stellar Evolution and $\mathrm{Nu}$ cleosynthesis (McGraw-Hill, New York).

Clayton, D. D., 1988, Mon. Not. R. Astron. Soc. 234, 1.

Clayton, D. D., 1996, "Radiogenic Iron," preprint (unpublished).

Clayton, D. D., S. A. Colgate, and G. J. Fishman, 1969, Astrophys. J. 155, 75.

Cottrell, P. L., and G. S. Da Costa, 1981, Astrophys. J. 245, L79.

Couch, R. G., A. B. Schmeidekamp, and W. D. Arnett, 1974, Astrophys. J. 190, 95.

Cowan, J. J., and D. L. Burris, 1997, private communication.

Cowan, J. J., D. L. Burris, C. Sneden, G. W. Preston, and A. McWilliam, 1995, Astrophys. J. 439, L51.

Cowan, J. J., A. McWilliam, C. Sneden, D. L. Burris, and G. W. Preston, 1997, Astrophys. J., in press.

Cowan, J. J., C. Sneden, J. W. Truran, and D. L. Burris, 1996, Astrophys. J. 460, L115.

Cowan, J. J., F.-K. Thielemann, and J. W. Truran, 1991, Phys. Rep. 208, 267.

Cox, A. N., 1965, Astrophys. J. Suppl. 11, 22.

Cox, A. N., and J. Stewart, 1970a, Astrophys. J. Suppl. 19, 243.

Cox, A. N., and J. Stewart, 1970b, Astrophys. J. Suppl. 19, 261.

Cox, A. N., J. Stewart, and D. D. Eilers, 1965, Astrophys. J. Suppl. 11, 1.

Cujec, B., and C. A. Barnes, 1976, Nucl. Phys. A 266, 461.

Cummings, A., and W. C. Haxton, 1996, Phys. Rev. Lett. 77, 4286.

Cunha, K., D. L. Lambert, M. Lemke, D. R. Gies, and L. C. Roberts, 1997, Astrophys. J., in press. 
Cunha, K., V. V. Smith, and D. L. Lambert, 1995, Astrophys. J. 452, 634.

Daudel, R., P. Benoist, R. Jaques, and M. Jean, 1947, C. R. Acad. Sci. 224, 1427.

Davis, Jr., R. 1964, Phys. Rev. Lett. 12, 303.

Davis, Jr., R., D. S. Harmer, and K. C. Hoffman, 1968, Phys. Rev. Lett. 20, 1205.

Dayras, R., Z. E. Switkowski, and S. E. Woosley, 1977, Nucl. Phys. A 279, 70.

Dearborn, D. S. P., 1992, Phys. Rep. 210, 367.

Dearborn, D. S. P., P. P. Eggleton, and D. N. Schram, 1976, Astrophys. J. 203, 455.

Dearborn, D. S. P., J. Liebert, M. Aaronson, C. C. Dahn, R. Harrington, J. Mould, and J. L. Greenstein, 1986, Astrophys. J. 300, 314.

Decrock, P., et al., 1991, Phys. Rev. Lett. 67, 808.

De Jager, C., 1984, Astron. Astrophys. 138, 246.

de Kool, M., and P. J. Green, 1995, Astrophys. J. 449, 236.

Deliyannis, C. P., 1990, Ph.D. Dissertation, Yale University.

Deliyannis, C. P., A. M. Boesgaard, and J. R. King, 1995, Astrophys. J. 452, L13.

Deliyannis, C. P., A. M. Boesgaard, J. R. King, and D. K. Duncan, 1997, Astron. J., submitted.

Deliyannis, C. P., P. Demarque, and S. D. Kawaler, 1990, Astrophys. J. 73, 21.

Deliyannis, C. P., J. R. King, and A. M. Boesgaard, 1996, preprint.

Deliyannis, C. P., J. R. King, A. M. Boesgaard, and S. G. Ryan, 1994, Astrophys. J. 434, L71.

Deliyannis, C. P., and R. A. Malaney, 1995, Astrophys. J. 453, 810.

Deliyannis, C. P., M. H. Pinsonneault, and D. K. Duncan, 1993, Astrophys. J. 414, 740.

Demarque, P., 1996, private communication.

Denissenkov, P. A., and S. N. Denissenkova, 1990, Sov. Astron. Lett. 16, 275.

Dennissenkov, P. A., A. Weiss, and J. Wagenhuber, 1997, Astron. Astrophys. 320, 115.

Denn, G. R., R. E. Luck, and D. L. Lambert, 1991, Astrophys. J. 377, 657.

Descouvemont, P., and D. Baye, 1994, Nucl. Phys. A 567, 341. Deutsch, A. J., 1956, Astrophys. J. 123, 210.

Dicus, D. A., 1972, Phys. Rev. D 6, 941.

Dicus, D. A.,E. W. Kolb, D. N. Schramm, and D. L. Tubbs, 1976, Astrophys. J. 210, 481.

Diehl, R., K. Bennett, H. Bloemen, C. Dupraz, W. Hermsen, J. Knödlseder, G. G. Lichti, D. Morris, U. Oberlack, J. Ryan, V. Schönfelder, H. Steinle, M. Varendorff, and C. Winkler, 1995, Astron. Astrophys. 298, L25.

Di Stefano, R., L. A. Nelson, W. Lee, J. H. Wood, and S. Rappaport, 1977, in Thermonuclear Supernovae, edited by R. Ruiz-Lapuente, R. Canal, and J. Isern (Kluwer, Dordrecht), p. 147.

Dominy, J. F., 1984, Astrophys. J. Suppl. 55, 27.

Dominy, J. F., 1985, Publ. Astron. Soc. Pac. 97, 1104.

Domogatskii, G. V., R. A. Eramzhyan, and D. K. Nadyozhin, 1977, in Neutrino 77 (Nauka, Moscow), p. 115.

Duerbeck, H. W., and S. Benetti, 1996, preprint.

Duncan, D. K., 1981, Astrophys. J. 248, 651.

Duncan, D. K., D. L. Lambert, and M. Lemke, 1992, Astrophys. J. 401, 584.

Duncan, D. K., R. C. Peterson, J. A. Thorburn, and M. H. Pinsonneault, 1994, Bull. Am. Astron. Soc. 26, 868.
Duncan, D. K., F. Primas, L. M. Rebull, A. M. Boesgaard, C. P. Deliyannis, L. M. Hobbs, J. R. King, and S. G. Ryan, 1997, Astrophys. J., in press.

Duncan, D. K., and L. Rebull, 1996, Publ. Astron. Soc. Pac. 108, 738.

Duncan, R. C., S. L. Shapiro, and I. Wasserman, 1986, Astrophys. J. 309, 141.

Dupraz, C., H. Bloeman, K. Bennett, R. Diehl, W. Hermsen, A. F. Iyudin, J. Ryan, and V. Schönfelder, 1996, Astron. Astrophys., in press.

Dyer, P., and C. A. Barnes, 1974, Nucl. Phys. A 233, 495.

Eberhardt, P., M. H. A. Junck, F. O. Meier, and F. Niederer, 1979, Astrophys. J. 234, L169.

Edvardsson, B., J. Andersen, B. Gustafsson, D. L. Lambert, P. E. Nissen, and J. Tomkin, 1993, Astron. Astrophys. 275, 101.

Edvardsson, B., B. Gustafsson, S. G. Johanssen, D. Kiselman, D. L. Lambert, P. E. Nissen, and G. Gilmore, 1994, Astron. Astrophys. 290, 176.

El Eid, M. F., 1994, Astron. Astrophys. 285, 915.

El Eid, M. F., and I. Baraffe, 1990, in Nuclei in the Cosmos, edited by $\mathrm{H}$. Oberhummer and W. Hillebrandt (MPI für Physik und Astrophysik, Garching), p. 238.

El Eid, M. F., and A. E. Champagne, 1995, Astrophys. J. 451, 298.

Elix, K., H. W. Becker, L. Buchmann, L. Görres, U. Kettner, M. Wiescher, and C. Rolfs, 1979, Z. Phys. A 293, 261.

Endt, P. M., 1990, Nucl. Phys. A 521, 1.

Endt, P. M., P. de Wit, and C. Alderliesten, 1986, Nucl. Phys. A 459, 61.

Endt, P. M., and C. Rolfs, 1987, Nucl. Phys. A 467, 261.

Engelbertink, G. A. P., and P. M. Endt, 1966, Nucl. Phys. 88, 12.

Erb, K. A., R. R. Betts, S. K. Korotky, M. M. Hindi, M. W. Sachs, S. J. Willet, and D. A. Bromley, 1980, Phys. Rev. C 22, 507.

Faulkner, J., 1971, Astrophys. J. 170, L99.

Federman, S. R., D. L. Lambert, J. A. Cardelli, Y. Sheffer, 1996, Nature (London) 381, 764.

Federman, S. R., Y. Sheffer, D. L. Lambert, and R. L. Gilliland, 1993, Astrophys. J. 413, L51.

Feltzing, S., and B. Gustafsson, 1994, Astrophys. J. 423, 68.

Festa, G. G., and M. Ruderman, 1969, Phys. Rev. 180, 1227.

Feynman, R. P., and M. Gell-Mann, 1958, Phys. Rev. 109, 193.

Filippone, B. W., J. Humblet, and K. Langanke, 1989, Phys.

Rev. C 40, 515.

Filippone, B. W., A. J. Elwyn, C. N. Davids, and D. D. Koetke, 1983a, Phys. Rev. Lett. 50, 412.

Filippone, B. W., A. J. Elwyn, C. N. Davids, and D. D. Koetke, 1983, Phys. Rev. C 28, 2222.

Ford, E., P. Kaaret, M. Tavani, D. Barret, P. Bloser, J. Grindlay, B. A. Harmom, W. S. Paciesas, and S. N. Zhang, 1997, Astrophys. J. 475, L123.

Forestini, M., and C. Charbonnel, 1997, Astron. Astrophys., in press.

Forestini, M., S. Goriely, A. Jorissen, and M. Arnould, 1992, Astron. Astrophys. 261, 157.

Fowler, W. A., G. R. Caughlin, and B. A. Zimmerman, 1975, Annu. Rev. Astron. Astrophys. 13, 69.

Fowler, W. A., and F. Hoyle, Astrophys. J. Suppl. 9, 201.

France III, R. H., E. L. Wilds, N. B. Jevtic, J. E. McDonald, and M. Gai, 1997, in Proceedings of Nuclei in the Cosmos IV (Nucl. Phys. A 621, 165c), and private communication.

François, P., 1986, Astron. Astrophys. 160, 264. 
François, P., M. Spite, and F. Spite, 1993, Astron. Astrophys. 274, 821.

Freedman, S. J., 1990, Comments Nucl. Part. Phys. 19, 209.

Fryer, C. L., W. Benz, and M. Herant, 1996, Astrophys. J. 460, 801.

Fujimoto, M. Y., 1977, Publ. Astron. Soc. Jpn. 29, 331.

Fujimoto, M. Y., 1988, Astron. Astrophys. 198, 163.

Fujimoto, M. Y., 1993, Astrophys. J. 419, 768.

Fujimoto, M. Y., and I. Iben, Jr., 1997, in Advances in Stellar Evolution, edited by R. T. Rood (Cambridge University Press, Cambridge), p. 245.

Fuller, G. M., W. A. Fowler, and M. J. Newman, 1980, Astrophys. J. Suppl. 42, 447.

Fuller, G. M., W. A. Fowler, and M. J. Newman, 1982a, Astrophys. J. 252, 715.

Fuller, G. M., W. A. Fowler, and M. J. Newman, 1982b, Astrophys. J. Suppl. Ser. 48, 279.

Fuller, G. M., W. A. Fowler, and M. J. Newman, 1985, Astrophys. J. 293, 1.

Fuller, G. M., R. W. Mayle, J. R. Wilson, and D. N. Schramm, 1987, Astrophys. J. 322, 795.

Fuller, G. M., and B. S. Meyer, 1995, Astrophys. J. 453, 792.

Galli, D., L. Stranghellini, M. Tosi, and F. Palla, 1996, Astrophys. J., in press.

Gallino, R., 1989, in The Evolution of Peculiar Red Giant Stars, edited by H. R. Johnson and B. Zuckerman (Cambridge University Press, Cambridge), p. 176.

Gallino, R., C. Arlandini, M. Lugaro, M. Busso, and O. Straniero, 1996, in Proceedings of Nuclei in the Cosmos IV (Nucl. Phys. A 621, 423c).

Gallino, R., M. Busso, G. Picchio, C. Raiteri, and A. Renzini, 1988, Astrophys. J. 334, L45.

Gallino, R., C. M. Raiteri, M. Busso, and F. Matteucci, 1994, Astrophys. J. 430, 858.

García-Berro, E., C. Ritossa, and I. Iben, Jr., 1997, Astrophys. J. 485, 765 .

Garcia-Lopez, R. J. , G. Severino, and M. T. Gomez, 1995, Astron. Astrophys. 297, 787.

Gass, H., J. Liebert, and R. Wehrse, 1988, Astron. Astrophys. 189, 194.

Gialanella, G., et al., 1996, Z. Phys. A 356, 107.

Gies, D. R., and D. L. Lambert, 1992, Astrophys. J. 387, 673.

Gilmore, G., B. Edvardsson, and P. E. Nissen, 1991, Astrophys. J. 378, 17.

Gilmore, G., B. Gustafsson, B. Edvardsson, and P. E. Nissen, 1992, Nature (London) 357, 379.

Gilroy, K. K., 1989, Astrophys. J. 347, 835.

Gilroy, K. K., and J. A. Brown, 1991, Astrophys. J. 371, 578.

Gilroy, K. K., C. Sneden, C. A. Pilachowski, and J. J. Cowan, 1988, Astrophys. J. 327, 298.

Glasner, S. A., and E. Livne, 1995, Astrophys. J. 445, L149.

Gonzalez, G., and G. Wallerstein, 1994, Astron. J. 108, 1325.

Görres, J., H. W. Becker, L. Buchmann, C. Rolfs, P. Schmalbrock, H.-P. Trautvetter, A. Vlieks, J. W. Hammer, and T. R. Donogue, 1983, Nucl. Phys. A 408, 372.

Görres, J., C. Rolfs, P. Schmalbrock, H.-P. Trautvetter, and J. Keinonen, 1982, Nucl. Phys. A 385, 57.

Görres, J., M. Wiescher, and C. Rolfs, 1989, Astrophys. J. 343, 365.

Gosh, P., and F. K. Lamb, 1992, in X-Ray Binaries and Recycled Pulsars, edited by E. P. J. van den Heuvel and S. A. Rappaport (Kluwer, Dordrecht), p. 487.
Gosh, P., F. K. Lamb, and C. J. Pethick, 1977, Astrophys. J. 217, 578 .

Gratton, R. G., and C. Sneden, 1991, Astron. Astrophys. 241, 501.

Gratton, R. G., and C. Sneden, 1994, Astron. Astrophys. 287, 927.

Green, P. J., B. Margon, S. F. Anderson, and D. J. MacConnell, 1992, Astrophys. J. 400, 659.

Green, P. J., B. Margon, and D. J. MacConnell, 1991, Astrophys. J. 380, L31.

Greenstein, J. L., 1954, in Modern Physics for Engineers, edited by J. L. Ridenour (McGraw-Hill, New York).

Greenstein, J. L., and R. S. Richardson, 1951, Astrophys. J. 113, 536.

Greenstein, J. L., and E. Tandberg-Hanssen, 1954, Astrophys. J. 119, 113.

Grevesse, N., Noels, Arletter, and A. J. Sauval, 1996, in Cosmic Abundances, edited by S. S. Holt and G. Sonneborn, ASP Conf. Ser. 99, 117.

Griffin, R., R. Griffin, B. Gustafsson, and T. Viera, 1982, Mon. Not. R. Astron. Soc. 198, 637.

Gustafsson, B., and N. Ryde, 1997, The Carbon Star Phenomenon, ASP Conf. Ser. (in press).

Gutiérrez, J., E. García-Berro, I. Iben, Jr., J. Isern, J. Labay, and R. Canal, 1996, Astrophys. J. 459, 701.

Hale, G. M., 1997, in Proceedings of Nuclei in the Cosmos IV, (Nucl. Phys. A 621, 177c).

Hansper, V. Y., S. G. Tims, A. J. Morton, A. F. Scott, C. I. W. Tingwell, and D. S. Sargood, in Proceedings of Nuclei in the Cosmos IV (Nucl. Phys. A 621, 285c).

Hardy, J., I. S. Towner, V. T. Koslowsky, E. Hagberg, and H. Schmeing, 1990, Nucl. Phys. A 509, 429.

Härm, R., and M. Schwarzschild, 1975, Astrophys. J. 200, 324.

Harper, C. L., 1996, Astrophys. J. 466, 1026.

Harper, C. L., and H. Wiesmann, 1992, Lunar Planet. Sci. XXIII, 489.

Harris, M. J., and D. L. Lambert, 1984, Astrophys. J. 281, 739.

Harris, M. J., and D. L. Lambert, 1987, Astrophys. J. 318, 868.

Harris, M. J., D. L. Lambert, and V. V. Smith, 1985, Astrophys. J. 299, 375.

Harris, M. J., D. L. Lambert, and V. V. Smith, 1988, Astrophys. J. 325, 768.

Hättig, H., K. Hünchen, and H. Wäffler, 1970, Phys. Rev. Lett. 25, 941.

Hatzes, A. P., 1987, Publ. Astron. Soc. Pac. 99, 369.

Hauser, W., and H. Feshbach, 1952, Phys. Rev. 87, 366.

Haxton, W. C., 1995, Annu. Rev. Astron. Astrophys. 33, 459.

Haxton, W. C., K. Langanke, Y.-Z. Qian, and P. Vogel, 1997, Phys. Rev. Lett. 78, 2694.

Hazard, C., B. Terlevich, D. C. Morton, W. L. W. Sargent, and G. Ferland, 1980, Nature (London) 285, 463.

Helfer, H. L., G. Wallerstein, and J. L. Greenstein, 1959, Astrophys. J. 129, 700.

Henyey, L. G., J. E. Forbes, and N. L. Gould, 1964, Astrophys. J. 139, 306.

Herant, M., W. Benz, and S. A. Colgate, 1992, Astrophys. J. 395, 642 .

Herbig, G. H., 1965, Astrophys. J. 141, 588.

Herndl, H., J. Görres, M. Wiescher, B. A. Brown, and L. van Wormer, 1995, Phys. Rev. C 52, 1078.

Herwig, S., T. Blöcker, D. Schonberner, and M. El Eid, 1997, Astron Astrophys., in press.

High, M. D., and B. Cujec, 1977, Nucl. Phys. A 282, 181. 
Hilgemeier, M., H. W. Becker, C. Rolfs, H. P. Trautvetter, and J. W. Hammer, 1988, Z. Phys. A 329, 243.

Hillebrandt, W., 1978, Space Sci. Rev. 21, 639.

Hix, W. R., and F.-K. Thielemann, 1996, Astrophys. J. 460, 869.

Hobbs, L. M., and D. K. Duncan, 1987, Astrophys. J. 317, 796. Hoffman, R. D., S. E. Woosley, G. M. Fuller, and B. S. Meyer, 1996, Astrophys. J. 460, 478.

Hoffman, R. D., S. E. Woosley, and Y.-Z. Qian, 1997, Astrophys. J. 482, in press.

Höflich, P., and A. Khokhlov, 1996, Astrophys. J. 457, 500.

Höflich, P., A. Khokhlov, J. C. Wheeler, M. M. Phillips, N. B. Suntzeff, and M. Hamuy, 1996, Astrophys. J. 472, L81.

Hogan, C. J., 1995, Astrophys. J. Lett. 441, L17.

Holberg, J. B., R. A. Saffer, R. W. Tweedy, and M. A. Bastrow, 1995, Astrophys. J. 452, L133.

Hollowell, D., and I. Iben, Jr., 1988, Astrophys. J. Lett. 333, L25.

Hollowell, D., and I. Iben, Jr., 1989, Astrophys. J. 340, 966.

Holmes, J. A., S. E. Woosley, W. A. Fowler, and B. A. Zimmerman, 1976, At. Data Nucl. Data Tables 18, 305.

Hoppe, P., P. S. Amari, E. Zinner, T. Ireland, and R. S. Lewis, 1994, Astrophys. J. 430, 870.

Hoppe, P., R. Strebel, P. Eberhardt, S. Amari, and R. S. Lewis, 1996, Science 272, 1314.

Howard, W. M., B. S. Meyer, and S. E. Woosley, 1991, Astrophys. J. 373, L5.

Hoyle, F., 1946, Mon. Not. R. Astron. Soc. 106, 343.

Hoyle, F., 1956, Astrophys. J. 124, 482.

Hoyle, F., D. N. F. Dunbar, W. A. Wenzel, and W. Whaling, 1953, Phys. Rev. 92, 1095.

Hoyle, F., and W. A. Fowler, 1960, Astrophys. J. 132, 565.

Hoyle, F., and M. Schwarzschild, 1955, Astrophys. J. Suppl. 2, 1.

Hubbard, W. B., and M. Lampe, 1969, Astrophys. J. Suppl. 18, 279.

Hulke, G., C. Rolfs, and H. P. Trautvetter, 1980, Z. Phys. A 297, 161.

Hulse, R. A., and J. H. Taylor, 1975, Astrophys. J. 195, L51.

Humblet, J., B. W. Filippone, and S. E. Koonin, 1991, Phys.

Rev. C 44, 2530.

Humphreys, R. M., 1978, Astrophys. J. Suppl. 38, 309.

Humphreys, R. M., and K. Davidson, 1979, Astrophys. J. 232, 409.

Humphreys, R. M., and K. Davidson, 1994, Publ. Astron. Soc. Pac. 106, 1025.

Iben, Jr., I., 1964, Astrophys. J. 140, 1631.

Iben, Jr., I., 1965, Astrophys. J. 142, 1447.

Iben, Jr., I., 1967a, Annu. Rev. Astron. Astrophys. 5, 571.

Iben, Jr., I., 1967b, Astrophys. J. 147, 650.

Iben, Jr., I., 1972, Astrophys. J. 178, 443.

Iben, Jr., I., 1975a, Astrophys. J. 196, 525.

Iben, Jr., I., 1975b, Astrophys. J. 196, 549.

Iben, Jr., I., 1976, Astrophys. J. 208, 165.

Iben, Jr., I., 1977a, Astrophys. J. 217, 788.

Iben, I., Jr., 1977b, in Advanced Stages of Stellar Evolution, edited by P. Bouvier and A. Maeder (Geneva Observatory, Sauverny), p. 1.

Iben, Jr., I., 1981, Astrophys. J. 246, 278.

Iben, Jr., I., 1982, Astrophys. J. 259, 244.

Iben, Jr., I., 1983, Astrophys. J. 275, L65.

Iben, Jr., I., 1984, Astrophys. J. 277, 333.

Iben, Jr., I., 1991a, Astrophys. J. Suppl. Ser. 76, 55.
Iben, I., Jr., 1991b, in Evolution of Stars: The Photospheric Abundance Connection, edited by G. Michaud and A. V. Tutukov (Dordrecht, Kluwer), p. 257.

Iben, J., Jr., 1997, in Thermonuclear Supernovea, edited by P. Ruiz-Lapuente, R. Canal, and J. Isern (Kluwer, Dordrecht), p. 111.

Iben, Jr., I., M. Y. Fujimoto, and J. MacDonald, 1991, Astrophys. J. 384, 580.

Iben, Jr., I., J. B. Kaler, J. W. Truran, and A. Renzini, 1983, Astrophys. J. 264, 605.

Iben, Jr., I., and M. Livio, 1993, Publ. Astron. Soc. Pac. 105, 1373.

Iben, I., Jr., and J. MacDonald, 1995, in White Dwarfs, edited by D. Koester and K. Warner (Springer, Berlin).

Iben, Jr., I., and A. Renzini, 1982a, Astrophys. J. 259, L79.

Iben, Jr., I., and A. Renzini, 1982b, Astrophys. J. Lett. 263, L23.

Iben, Jr., I., and A. Renzini, 1983, Annu. Rev. Astron. Astrophys. 21, 271.

Iben, Jr., I., C. Ritossa, and E. García-Berro, 1997, Astrophys. $\mathrm{J}$. , in press.

Iben, Jr., I., and J. W. Truran, 1978, Astrophys. J. 220, 980.

Iben, Jr., I., and A. V. Tutukov, 1984, Astrophys. J. Suppl. Ser. 54, 335.

Iben, Jr., I., and A. V. Tutukov, 1985, Astrophys. J. Suppl. Ser. 58, 661.

Iben, Jr., I., and A. V. Tutukov, 1989, Astrophys. J. 342, 430.

Iben, Jr., I., and A. V. Tutukov, 1996, Astrophys. J. Suppl. Ser. $\mathbf{1 0 5}, 145$.

Iben, Jr., I, and A. V. Tutokov, 1997, Astrophys. J. Suppl. Ser. 491, in press.

Iben, Jr., I., A. V. Tutukov, and L. R. Yungelson, 1995, Astrophys. J. Suppl. Ser. 100, 233.

Iben, Jr., I., A. V. Tutukov, and L. R. Yungelson, 1996, Astrophys. J. 456, 750.

Iben, Jr., I., A. V. Tutukov, and L. R. Yungelson, 1997, Astrophys. J. 475, 291.

Iglesias, C. A., and F. J. Rogers, 1996, Astrophys. J. 464, 943. Iglesias, C. A., F. J. Rogers, and B. G. Wilson, 1990, Astrophys. J. 360, 221.

Iliadis, C., 1996, private communication.

Iliadis, C., et al., 1990, Nucl. Phys. A 512, 509.

Imhof, W. L., R. G. Johnson, F. J. Vaughn, and M. Walt, 1959, Phys. Rev. C 114, 1037.

INT Workshop on Solar Nuclear Fusion Rates, 1997, Rev. Mod. Phys. (to be published).

Ireland, T. R., and W. Compston, 1987, Nature (London) 327, 689.

Ito, K., 1961, Prog. Theor. Phys. 26, 990.

Itoh, N., H. Hayashi, A. Nishikawa, and Y. Kohyama, 1996, Astrophys. J. Suppl. Ser. 102, 411.

Jaag, S., 1990, master thesis, University of Karlsruhe.

Jaag, S., and F. Käppeler, 1995, Phys. Rev. C 51, 3465.

Jaag, S., and F. Käppeler, 1996, Astrophys. J. 464, 874.

Jacoby, G., and H. Ford, 1983, Astrophys. J. 266, 298.

Johnson, C. W., E. Kolbe, S. E. Koonin, and K. Langanke, 1992, Astrophys. J. 392, 320.

Jorissen, A., D. T. Frayer, H. R. Johnson, M. Mayor, and V. V. Smith, 1993, Astron. Astrophys. 271, 463.

Jorissen, A., V. V. Smith, and D. L. Lambert, 1992, Astron. Astrophys. 251, 164.

Jung, M., F. Bosch, K. Beckert, H. Eickhoff, H. Folger, B. Franzke, A. Gruber, P. Kienle, O. Klepper, W. Koenig, C. 
Kozhuharov, R. Mann, R. Moshammer, F. Nolden, U. Schaaf, G. Soff, P. Spädke, M. Steck, T. Stöhlker, and K. Sümmerer, 1992, Phys. Rev. Lett. 69, 2164.

Jura, M., D. M. Meyer, I. Hawkins, and J. A. Cardelli, 1996, Astrophys. J. 456, 598.

Kahane, C., J. Cernicharo, J. Gomez-Gonzalés, and M. Guélin, 1992, Astron. Astrophys. 256, 235.

Kajino, T., H. Toki, and S. M. Austin, 1987, Astrophys. J. 319, 531.

Kamkionkowski, M., and J. N. Bahcall, 1994, Astrophys. J. 420, 884.

Käppeler, F., 1992, in Radioactive Nuclear Beams, edited by T. D. A. Hilger, Bristol, p. 305.

Käppeler, F., H. Beer, and K. Wisshak, 1989, Rep. Prog. Phys. 52, 945 .

Käppeler, F., R. Gallino, M. Busso, G. Picchio, and C. M. Raiteri, 1990, Astrophys. J. 354, 630.

Käppeler, F., S. Jaag, Z. Bao, and G. Reffo, 1991, Astrophys. J. 366, 605.

Käppeler, F., W. Schanz, K. Wisshak, and G. Reffo, 1993, Astrophys. J. 410, 370.

Kato, M., 1983, Publ. Astron. Soc. Jpn. 35, 507.

Kato, M., and I. Hachisu, 1994, Astrophys. J. 437, 296.

Keenan, P. C., 1942, Astrophys. J. 96, 101.

Keenan, P. C., 1954, Astrophys. J. 120, 484.

Keinonen, J., and S. Brandenburg, 1980, Nucl. Phys. A 341, 345.

Keinonen, J., M. Riihonen, and A. Anttila, 1977, Phys. Rev. C 15, 579 .

Keller, G., and R. E. Meyerott, 1955, Astrophys. J. 122, 32.

Kettner, K. U., H. Lorentz-Wirzba, and C. Rolfs, 1980, Z. Phys. A 298, 65.

Kiener, J., et al., 1993, Nucl. Phys. A 552, 66.

Kiener, J., V. Tatischeff, P. Aguer, G. Bogaert, A. Coc, D. Disdier, L. Kraus, A. Lefebvre, I. Linck, W. Mittif, T. Motobayashi, F. de Oliveira-Santos, P. Roussel-Chomaz, C. Stephan, and J. P. Thibaud, in Proceedings of Nuclei in the Cosmos IV (Nucl. Phys. A 621, 173c).

King, J. R., 1993, Astron. J. 105, 1087.

King, J. R., C. P. Deliyannis, and A. M. Boesgaard, 1996, Astron. J. 112, 2839.

King, J. R., C. P. Deliyannis, and A. M. Boesgaard, 1997, Astrophys. J. 478, 778.

King, J. R., C. P. Deliyannis, D. D. Hiltgen, A. Stephens, K. Cunha, and A. M. Boesgaard, 1997, Astron. J., 113, 1871.

Kiselman, D., 1994, Astron. Astrophys. 286, 169.

Kiselman, D., and M. Carlsson, 1995, in The Light Element Abundances, Isola d'Elba, ESO/EIPC Workshop, edited by Phillipe Crane (Springer, Berlin), p. 372.

Kiselman, D., and M. Carlsson, 1996, Astron. Astrophys. 311, 680.

Klay, N., and F. Käppeler, 1988, Phys. Rev. C 38, 295.

Klay, N., F. Käppeler, H. Beer, and G. Schatz, 1991, Phys. Rev. C 44, 2839.

Koehler, P. E., F. Käppeler, H. Schatz, Yu. M. Gledenov, Yu. P. Popov, J. A. Harvey, N. W. Hill, M. Wiescher, R. W. Kavanagh, and R. B. Vogellar, 1994, in Capture Gamma-Ray Spectroscopy and Related Topics, edited by J. Kern (World Scientific, Singapore), pp. 714-723.

Koehler, P., R. Spencer, R. Winters, K. Guber, J. Harvey, N. Hill, and M. Smith, 1996, Phys. Rev. C 54, 1463.

Kohl, J. L., W. H. Parkinson, and G. L. Withbroe, 1977, Astrophys. J. 212, 101.
Koonin, S. E., T. A. Tombrello, and G. Fox, 1974, Nucl. Phys. A 220, 221.

Kovacs, N., 1985, Astron. Astrophys. 150, 232.

Kraft, R. P., 1994, Publ. Astron. Soc. Pac. 106, 553.

Kraft, R. P., J. Mathews, and J. L. Greenstein, 1962, Astrophys. J. 136, 312.

Kraft, R. P., C. Sneden, G. E. Langer, and M. D. Shetrone, 1993, Astron. J. 106, 1490.

Kraft, R. P., C. Sneden, G. E. Langer, M. D. Shetrone, and M. Boltz, 1995, Astron. J. 109, 2586.

Kraft, R. P., C. Sneden, G. H. Smith, M. D. Shetrone, G. E. Langer, and C. A. Pilachowski, 1977, Astron. J. 113, 279.

Kraicheva, Z. T., E. I. Popova, A. V. Tutukov, and L. R. Yungelson, 1978, Astron. Zh. 55, 1176.

Kratz, K.-L., J.-P. Bitouzet, F.-K. Thielemann, P. Moller, and B. Pfeiffer, 1983, Astrophys. J. 403, 216.

Krauss, A., H. W. Becker, H. P. Trautvetter, and C. Rolfs, 1987, Nucl. Phys. A 467, 273.

Krauss, L. M., and P. Romanelli, 1990, Astrophys. J. 358, 47.

Kräwinkel, H., et al., 1982, Z. Phys. A 304, 307.

Kremer, R. M., C. A. Barnes, K. H. Chang, H. C. Evans, B. W. Fillipone, K. H. Hahn, and L. W. Mitchell, 1988, Phys. Rev. Lett. 60, 1475.

Kunth, D., M. Guiderdoni, M. Heydari-Malayeri, and T. X. Thuan, 1997, The Interplay between Massive Star Formation, the ISM, and Galaxy Evolution (Editions Frontiers, Gif-surYvette Cedex).

Kurfess, J. D., et al., 1992, Astrophys. J. Lett. 399, L137.

Kurucz, R. L., 1996, in Astron. Soc. of the Pac. Conf. Series, Vol. 108, edited by S. Adelman, F. Kupka, and W. W. Weiss. Laird, J. B., B. W. Carney, and D. W. Latham, 1988, Astron. J. 95, 1843.

Lamb, W. A. S., and R. E. Hester, 1957, Phys. Rev. 108, 1304.

Lamb, S. A., W. M. Howard, J. W. Truran, I. Iben, Jr., 1977, Astrophys. J. 217, 213.

Lamb, S. A., N. Shibazaki, M. A. Alpar, and J. Shalam, 1985, Nature (London) 317, 681.

Lambert, D. L., 1985, in Cool Stars with Excesses of Heavy Elements, edited by M. Jaschek and P. C. Keenan (Reidel, Dordrecht), p. 191.

Lambert, D. L., 1988, in The Impact of Very High S/N Spectroscopy on Stellar Physics, edited by G. Cayrel de Strobel and M. Spite (Kluwer, Dordrecht), p. 563.

Lambert, D. L., 1991, in Evolution of Stars: The Photospheric Abundance Connection, edited by G. Michaud and A. Tutukov (Kluwer, Dordrecht), p. 299.

Lambert, D. L., 1992, Astron. Astrophys. Rev. 3, 201.

Lambert, D. L., 1995, Astron. Astrophys. 301, 478.

Lambert, D. L., J. F. Dominy, and S. Silvertsen, 1980, Astrophys. J. 235, 114.

Lambert, D. L., B. Gustafsson, K. Eriksson, and K. H. Hinkle, 1986, Astrophys. J. Suppl. Ser. 62, 373.

Lambert, D. L., J. E. Heath, and B. Edvardsson, 1991, Mon. Not. R. Astron. Soc. 253, 610.

Lambert, D. L., and L. M. Ries, 1981, Astrophys. J. 248, 228.

Lambert, D. L., V. V. Smith, M. Busso, R. Gallino, and O. Straniero, 1995, Astrophys. J. 450, 302.

Langanke, K., 1994, private communication.

Langer, N., 1989a, Astron. Astrophys. 210, 93.

Langer, N., 1989b, Astron. Astrophys. 220, 135.

Langer, N., J.-P. Arcoragi, and M. Arnould, 1989, Astron. Astrophys. 210, 187. 
Lawson, W. A., P. L. Cottrell, P. M. Kilmartin, and A. C. Gilmore, 1990, Mon. Not. R. Astron. Soc. 247, 91.

Lee, C. W., E. L. Martin, and R. D. Mattieu, 1994, Astron. J. 108, 1445 .

Lee, T., D. A. Papanastassiou, and G. J. Wasserburg, 1977, Astrophys. J. 211, L107.

Leep, E. M., G. Wallerstein, and J. B. Oke, 1986, Astrophys. J. 91, 1117.

Leising, M. D., and G. H. Share, 1990, Astrophys. J. 357, 638. Leising, M. D., and G. H. Share, 1994, Astrophys. J. 424, 200. Lemke, M., D. L. Lambert, and B. Edvardsson, 1993, Publ. Astron. Soc. Pac. 105, 468.

Lemoine, M., M. Cassé, and E. Vangioni-Flam, 1977, Astrophys. J. , in press.

Lemoine, M., R. Ferlet, and A. Vidal-Madjar, 1995, Astron. Astrophys. 298, 879.

Lewis, R. S., L. Alaerts, I. Matsuda, and E. Anders, 1979, Astrophys. J. 234, L165.

Liebert, J., and P. Bergeron, 1995, in White Dwarfs, edited by

K. Werner and D. Koester (Berlin, Springer), p. 12.

Lingenfelter, R. E., and R. Ramaty, 1989, Astrophys. J. 343, 686.

Little-Marenin, I. R., 1989, in Evolution of Peculiar Red Giants, edited by H. R. Johnson and B. Zuckerman (Cambridge University Press, Cambridge), p. 131.

Livio, M., A. Shankar, A. Burkert, and J. W. Truran, 1990, Astrophys. J. 356, 250.

Livne, E., and W. D. Arnett, 1995, Astrophys. J. 452, 62.

Lloyd Evans, T., 1983, Mon. Not. R. Astron. Soc. 204, 975.

Lorimer, D. R., M. Bailes, R. J. Dewey, and P. A. Harrison, 1993, Mon. Not. R. Astron. Soc. 263, 403.

Loss, R. D., and G. W. Lugmair, 1990, Astrophys. J. Lett. 360, L59.

Luck, R. E., and H. E. Bond, 1982, Astrophys. J. 259, 792.

Luck, R. E., and D. L. Lambert, 1982, Astrophys. J. 256, 189. Lyubimkov, L. S., 1993, Astron. Soc. Pac. Conf. Ser. 40, 183.

MacCallum, C. J., A. F. Huters, P. D. Stang, and M. Leventhal, 1987, Astrophys. J. 317, 877.

MacDonald, J., 1980, Mon. Not. R. Astron. Soc. 191, 933.

MacDonald, J., 1986, Astrophys. J. 305, 251.

MacDonald, J., 1992, Astrophys. J. 394, 619.

Macklin, R. L., and J. H. Gibbons, 1967, Astrophys. J. 149, 577.

Maeder, A., 1992, Astron. Astrophys. 264, 105.

Magain, P., 1989, Astron. Astrophys. 209, 211.

Magain, P., 1995, Astron. Astrophys. 297, 686.

Mahoney,W. A., et al., 1988, Astrophys. J. 334, L81.

Mahoney, W. A., J. C. Ling, W. A. Wheaton, and A. S. Jacobson, 1984, Astrophys. J. 286, 578.

Malaney, R. A., 1987, Astrophys. J. 321, 832.

Malaney, R. A., and M. N. Butler, 1993, Astrophys. J. 407, L73.

Malaney, R. A., and W. A. Fowler, 1987, in Origin and Distribution of the Elements, edited by G. J. Mathews (World Scientific, Singapore), pp. 76-85.

Malaney, R. A., and W. A. Fowler, 1988, Astrophys. J. 333, 13. Malaney, R. A., and D. L. Lambert, 1988, Mon. Not. R. Astron. Soc. 235, 695.

Mann, F.M., 1976, HAUSER*4 code, Hanford Engineering Development Laboratory, Report HEDL-76-80.

Mann, F. M., 1978, HAUSER*5 code, Report HEDL-78-83.

Marion, J. B., and W. A. Fowler, 1957, Astrophys. J. 125, 221. Massevich, A. G., E. I. Popova, A. V. Tutukov, and L. R. Yungelson, 1979, Astrophys. Space Sci. 62, 451.
Mathews, G. J., and R. A. Ward, 1985, Rep. Prog. Phys. 48, 1371.

Matteucci, F., 1989, in Evolutionary Phenomena in Galaxies, edited by J. Beckmann and B. Pagel (Cambridge University Press, Cambridge), p. 341.

Matz, S. M., G. H. Share, M. D. Leising, E. L. Chupp, W. T. Vestrand, et al., 1988, Nature (London) 331, 416.

Mazarakis, M., and W. Stephens, 1972, Astrophys. J. 171, L97. Mazarakis, M., and W. Stephens, 1973, Phys. Rev. C 7, 1280.

McClure, R. D., 1984, Astrophys. J. Lett. 280, L31.

McClure, R. D., 1985, in Cool Stars with Excesses of Heavy Elements, edited by M. Jaschek and P. C. Keenan (Reidel, Dordrecht), p. 315.

McClure, R. D., 1989, in Evolution of Peculiar Red Giants, edited by H. R. Johnson and B. Zuckerman (Cambridge University Press, Cambridge), p. 196.

McClure, R. D., 1997, Publ. Astron. Soc. Pac., in press.

McClure, R. D., J. M. Fletcher, and J. M. Nemec, 1980, Astrophys. J. Lett. 238, L35.

McClure, R. D., and A. W. Woodsworth, 1990, Astrophys. J. 352, 709.

McDonald, A. B., T. K. Alexander, J. E. Beene, and H. B. Mak, 1977, Nucl. Phys. A 288, 529.

McLaughlin, G. C., G. M. Fuller, and J. R. Wilson, 1996, Astrophys. J. 472, 440.

McWilliam, A., G. W. Preston, C. Sneden, and L. Searle, 1995, Astron. J. 109, 2757.

Meneguzzi, M., J. Audouze, and H. Reeves, 1971, Astron. Astrophys. 15, 337.

Mengel, J. G., and A. V. Sweigart, 1981, in Astrophysical Parameters for Globular Clusters, edited by A. G. D. Philip (Reidel, Dordrecht), p. 277.

Merrill, P. W., 1922, Astrophys. J. 56, 457.

Merrill, P. W., 1952, Astrophys. J. 116, 21.

Merrill, P. W., 1956, Publ. Astron. Soc. Pac. 68, 70.

Meyer, B. S., 1994, Annu. Rev. Astron. Astrophys. 32, 153.

Meyer, B. S., T. D. Krishnan, and D. D. Clayton, 1966, Astrophys. J. 462, 865 .

Meyer, B. S., T. D. Krishnan, and D. D. Clayton, 1997, Astrophys. J., in press.

Mezzacappa, A., and S. Bruenn, 1993, Astrophys. J. 405, 637. Michaud, G., 1972, Astrophys. J. 175, 751.

Michaud, G., 1986, Astrophys. J. 302, 650.

Mikheyev, S. P., and A. Y. Smirnov, 1985, Sov. J. Nucl. Phys. 42, 913.

Miyaji, S., and K. Nomoto, 1987, Astrophys. J. 318, 307.

Miyaji, S., K. Nomoto, K. Yokoi, and D. Sugimoto, 1980, Publ. Astron. Soc. Pac. 32, 303.

Mochkovich, R., and M. Livio, 1990, Astron. Astrophys. 236, 378.

Mohar, M. F., D. Bazin, W. Benenson, D. J. Morrissey, N. A. Orr, B. M. Sherrill, D. Swan, J. A. Winger, A. C. Mueller, and D. Guillemaud-Mueller, 1991, Phys. Rev. Lett. 66, 1571.

Molaro, P., and L. Pasquini, 1994, Astron. Astrophys. 281, L77.

Molaro, P., F. Primas, and P. Bonifacio, 1995, Astron. Astrophys. 295, 47.

Möller, P., J. R. Nix, W. D. Meyers, and W. J. Swiatecki, 1995, At. Data Nucl. Data Tables 59, 185.

Morell, O., D. Källander, and H. R. Butcher, 1992, Astron. Astrophys. 259, 543.

Morgan, E. H., and D. A. Smith, 1996, IAV Circ. 6437.

Morgan, S., and G. Lake, 1989, Astrophys. J. 339, 71.

Motobayashi, T., et al., 1991, Phys. Lett. B 264, 259. 
Motobayashi, T., et al., 1994, Phys. Rev. Lett. 73, 2680.

Myra, E. S., and A. Burrows, 1990, Astrophys. J. 364, 222.

Nagai, Y., M. Igashira, N. Mugai, T. Ohsaki, F. Uesawa, K.

Takeda, T. Ando, H. Kitazawa, S. Kubono, and T. Fukuda, 1991, Astrophys. J. 381, 444.

Nagatani, K., M. R. Dwarakanath, and D. Ashery, 1969, Nucl. Phys. A 128, 325.

Neubeck, K., H. Schober, and H. Wäffler, 1974, Phys. Rev. C 10, 320 .

Niemeyer, J. C., W. Hillebrandt, and S. E. Woosley, 1996, Astrophys. J. 471, 903.

Niemeyer, J. C., and S. E. Woosley, 1997, Astrophys. J. 475, 740.

Niemeyer, S., 1988, Geochim. Cosmochim. Acta 28, 2941.

Nittler, L. R., C. M. O'D. Alexander, X. Gao, R. M. Walker, and E. K. Zinner, 1994, Nature (London) 370, 443.

Nomoto, K., 1984, Astrophys. J. 277, 791.

Nomoto, K., 1987, Astrophys. J. 322, 206.

Nomoto, K., and M. Hashimoto, 1988, Phys. Rep. 163, 13.

Nomoto, K., and I. Iben, Jr., 1985, Astrophys. J. 297, 531.

Nomoto, K., K. Iwamoto, N. Nakasato, F.-K. Thielmann, F. Brachwitz, T. Young, T. Sigeyama, T. Tsujimoto, and Y. Yoshii, 1977, in Thermonuclear Supernovae, edited by P. Ruiz-Lapuente, R. Canal, and J. Isern (Kluwer, Dordrecht). Nomoto, K., F.-K. Thielmann, and Yokoi, 1984, Astrophys. J. 286, 644.

Norris, J. E., and G. S. Da Costa, 1995, Astrophys. J. 447, 680. North, P., S. Berthet, and T. Lanz, 1994, Astron. Astrophys. 281, 775.

Novikov, I. D., and Ya. B. Zeldovich, 1966, Nuovo Cimento 4, 810.

Ohnaka, K., and T. Tsuji, 1996, Astron. Astrophys. 310, 933.

Olive, K., and D. N. Schramm, 1996, Phys. Rev. D 54, 109.

Opik, J., 1951, Proc. R. Irish Acad. A. 54, 49.

Osborne, J. L., C. A. Barnes, R. W. Kavanagh, R. M. Kremer, G. J. Mathews, J. L. Zyskind, P. D. Parker, and A. J. Howard, 1982, Phys. Rev. Lett. 48, 1664.

Osborne, J. L., C. A. Barnes, R. W. Kavanagh, R. M. Kremer, G. J. Matthews, J. L. Zyskind, P. D. Parker, and A. J. Howard, 1984, Nucl. Phys. A 419, 115.

Ouellet, J. M. L., M. N. Butler, H. C. Evans, H. W. Lee, J. R. Leslie, J. D. McArthur, W. McLatchie, H.-B. Mak, P. Skensved, J. L. Whitton, and X. Zhao, 1996, Phys. Rev. C 54, 1982.

Ouellet, J. M. L., H. C. Evans, H. W. Lee, J. R. Leslie, J. D. McArthur, W. McLatchie, H.-B. Mak, P. Skensved, X. Zhao, and T. K. Alexander, 1992, Phys. Rev. Lett. 69, 1896.

Paczyński, B., 1967, Acta Astron. 17, 287.

Paczyński, B., 1970, Acta Astron. 20, 47.

Paczyński, B., 1971a, Acta Astron. 21, 417.

Paczyński B., 1971b, Annu. Rev. Astron. Astrophys. 9, 183.

Paczyński, B., 1976, in The Structure and Evolution of Close Binary Systems, edited by P. Eggleton, S. Mitton, and J. Whelan (Reidel, Dordrecht), p. 75.

Padgett, D. L., 1990, ASP Conf. Series, Vol. 9, p. 355.

Padgett, D. L., 1991, Ph. D. thesis, Caltech.

Pagel, B. E. J., 1965, Roy. Obs. Bull., No. 104.

Pagel, B. E. J., 1989, in Evolutionary Phenomena in Galaxies, edited by J. Beckman and B. E. J. Pagel (Cambridge University Press, Cambridge), p. 201.

Pagel, B. E. J., 1997, Nucleosynthesis and Chemical Evolution of Galaxies (Cambridge University Press, Cambridge, England).
Paine, B. M., and D. G. Sargood, 1979, Nucl. Phys. A 331, 389. Parker, P. D., and R. W. Kavanagh, 1963, Phys. Rev. 131, 2578. Pasquini, L., and P. Molaro, 1996, Astron. Astrophys. 307, 761. Patterson, J. R., H. Winkler, and C. Zaidins, 1969, Astrophys. J. 157, 367.

Peters, J. G., 1968, Astrophys. J. 154, 225.

Peters, J. G., W. A. Fowler, and D. D. Clayton, 1972, Astrophys. J. 173, 637.

Peterson, R. C., 1980, Astrophys. J. 237, L87.

Peterson, R. C., R. L. Kurucz, and B. W. Carney, 1990, Astrophys. J. 350, 173.

Pilachowski, C., 1986, Astrophys. J. 300, 289.

Pilachowski, C. A., G. Wallerstein, and E. M. Leep, 1980, Astron. J. 236, 508.

Pinsonneault, M. H., C. P. Deliyannis, and P. Demarque, 1992, Astrophys. J. 78, 181.

Pinsonneault, M. H., S. D. Kawalar, and P. Demarque, 1990, Astrophys. J. 74, 501.

Plaga, R., H. W. Becker, A. Redder, C. Rolfs, H. P. Trautvetter, and K. Langanke, 1987, Nucl. Phys. A 465, 291.

Plaga, R., 1986, Diploma thesis, University of Münster.

Plez, B., V. V. Smith, and D. L. Lambert, 1993, Astrophys. J. 418, 812 .

Politano, M., S. Starrfield, J. W. Truran, A. Weiss, and W. M. Sparks, 1995, Astrophys. J. 448, 807.

Pontecorvo, B., 1946, Chalk River Report PD-205 (unpublished).

Popova, E. I., A. V. Tutukov, and L. R. Yungelson, 1982, Astrophys. Space Sci. 88, 155.

Praderie, F., A. M. Boesgaard, B. Milliard, and M. L. Pitois, 1977, Astrophys. J. 214, 130.

Prantzos, N., O. Aubert, and J. Audouze, 1996, Astron. Astrophys. 309, 760.

Prantzos, N., M. Cassé, and E. Vangioni-Flam, 1993, Astrophys. J. 403, 630.

Prantzos, N., M. Hashimoto, and K. Nomoto, 1990, Astron. Astrophys. 234, 211.

Prantzos, N., M. Hashimoto, M. Rayet, and M. Arnould, 1990, Astron. Astrophys. 238, 455.

Prialnik, D., and A. Kovetz, 1984, Astrophys. J. 281, 367.

Primas, F., 1996, Publ. Astron. Soc. Pac. 108, 724.

Qian, Y.-Z., and G. M. Fuller, 1995, Phys. Rev. D 51, 1479; 52, 656.

Qian, Y.-Z., and S. E. Woosley, 1996, Astrophys. J. 471, 331.

Raiteri, C., R. Gallino, M. Busso, D. Neuberger, and F. Käppeler, 1993, Astrophys. J. 419, 207.

Rao, N. K., and D. L. Lambert, 1996, in Hydrogen-Deficient Stars, edited by C. S. Jeffery and U. Heber (ASP, San Francisco), p. 43.

Rappaport, S. A., and E. P. J. van den Heuvel, 1982, in The Be Stars, edited by M. Jaschek and H. Groth (Reidel, Dordrecht), p. 327.

Rauscher, T., F. K. Thielemann, and K.-L. Kratz, 1997, in Proceedings of Nuclei in the Cosmos IV (Nucl. Phys. A 621, 331c).

Rayet, M., M. Arnould, M. Hasimoto, N. Prantzos, and K. Nomoto, 1995, Astron. Astrophys. 298, 517.

Rayet, M., N. Prantzos, and M. Arnould, 1990, Astron. Astrophys. 227, 271.

Razio, F. A., and M. Livio, 1996, Astrophys. J. 471, 366.

Rebolo, R., P. Molaro, C. Abia, and J. E. Beckman, 1988, Astron. Astrophys. 193, 193. 
Rebolo, R., P. Molaro, and J. E. Beckman, 1988, Astron. Astrophys. 192, 192.

Redder, A., H. W. Becker, C. Rolfs, H. P. Trautvetter, T. R. Donoghue, T. C. Rinkel, J. W. Hammer, and K. Langanke, 1987, Nucl. Phys. A 462, 385.

Reeves, H., W. A. Fowler, and F. Hoyle, 1970, Nature (London) 226, 727.

Reid, I. N., 1997, Astron J. 114, 161.

Reinhard, P. G., J. Friedrich, K. Goeke, F. Grümmer, and D. H. Gross, 1984, Phys. Rev. C 30, 878.

Rembges, F., F.-K. Thielemann, H. Schatz, J. Görres, and M. Wiescher, 1996, Proceedings of the Eighth Workshop on Nuclear Astrophysics, Ringberg Castle, Germany, 1996 (unpublished).

Renzini, A., 1981, in Physical Processes in Red Giants, edited by I. Iben, Jr., and A. Renzini (Reidel, Dordrecht), p. 431.

Renzini, A., 1990, in Confrontation Between Stellar Pulsation and Evolution, edited by C. Cacciari and G. Clementini (ASP, San Francisco), p. 549.

Renzini, A., and M. Voli, 1981, Astron. Astrophys. 94, 175.

Rester, A. C., et al., 1988, Astrophys. J. 342, L71.

Richard, O., S. Vauclair, C. Charbonnel, and W. A. Dziembrowski, 1996, Astron. Astrophys. 312, 1000.

Ritossa, C., E. García-Berro, and I. Iben, Jr., 1996, Astrophys. J. 460, 489.

Ritossa, C., E. Gracía-Berro, and I. Iben, Jr., 1997, Astrophys. $\mathrm{J}$. , in press.

Robertson, R. G. H., P. Dyer, T. J. Bowles, R. E. Brown, N. Jarmie, C. J. Maggiore, and S. M. Austin, 1983, Phys. Rev. C 27, 11.

Roby, R. W., and D. L. Lambert, 1990, Astrophys. J. Suppl. Ser. 73, 67.

Rogers, F. J., and C. A. Iglesias, 1992, Astrophys. J. Suppl. Ser. 79, 507.

Rolfs, C., and W. S. Rodney, 1975, Nucl. Phys. A 250, 295.

Rolfs, C., and W. S. Rodney, 1988, Cauldrons in the Cosmos (University of Chicago Press, Chicago).

Rolfs, C., W. S. Rodney, M. H. Shapiro, and H. Winkler, 1975, Nucl. Phys. A 241, 460.

Rollefson, A. A., V. Wijekumar, C. P. Browne, M. Wiescher, H. J. Hausman, W. Y. Kim, and P. Schmalbrock, 1990, Nucl. Phys. A 507, 413.

Rood, R. T., T. M. Bania, and T. L. Wilson, 1992, Nature (London) 355, 618.

Rood, R. T., T. M. Bania, T. L. Wilson, and D. S. Balsar, 1995, in The Light Element Abundances, edited by P. Crane (Springer, Berlin), p. 201.

Roughton, N. A., M. R. Fritts, R. J. Petersen, C. S. Zaidins, and C. J. Hansen, 1979, At. Data Nucl. Data Tables 23, 177.

Roughton, N. A., T. P. Intrator, R. J. Petersen, C. S. Zaidins, and C. J. Hansen, 1983, At. Data Nucl. Data Tables 28, 341.

Ruffert, M., H.-Th. Janka, K. Takahashi, and G. Schäfer, 1997, Astron. Astrophys. 319, 122.

Russell, S. S., G. Srinivasan, G. R. Huss, G. J. Wasserburg, and G. J. MacPherson, 1996, Science 273, 757.

Ryan, S. G., T. Beers, C. P. Deliyannis, and J. Thorburn, 1996, Astrophys. J. 458, 543.

Ryan, S. G., J. E. Norris, M. S. Bessell, and C. P. Deliyannis, 1992, Astrophys. J. 388, 184.

Ryter, C., H. Reeves, E. Gradsztajn, and J. Audouze, 1970, Astrophys. J. 388, 184.
Sackmann, I.-J., and A. I. Boothroyd, 1991, in Evolution of Stars: The Photospheric Abundance Connection, edited by G. Michaud and A. V. Tutukov (Kluwer, Dordrecht), p. 275.

Sackmann, I.-J., and A. I. Boothroyd, 1992, Astrophys. J. 392, L71.

Saio, H., and K. Nomoto, 1985, Astron. Astrophys. 150, L21. Salam, A., 1968, in Elementary Particle Physics, edited by N.

Swartholm (Almqvist, Stockholm).

Salpeter, E. E., 1952, Astrophys. J. 115, 326.

Salpeter, E. E., 1954, Aust. J. Phys. 7, 373.

Sanders, R. H., 1967, Astrophys. J. 150, 971.

Sandie, W. G., et al., 1988, Astrophys. J. 342, L91.

Sargood, D. G., 1982, Phys. Rep. 93, 63.

Savonije, G. J., 1979, Astron. Astrophys. 71, 352.

Scalo, J. M., K. H. Despain, and R. K. Ulrich, 1975, Astrophys. J. 196, 805.

Schaerer, D., C. Charbonnel, G. Meynet, A. Maeder, and G. Schaller, 1993, Astron. Astrophys. Suppl. Ser. 102, 339.

Schaerer, D., G. Meynet, A. Maeder, and G. Schaller, 1992, Astron. Astrophys. Suppl. Ser. 98, 523.

Schaller, G., D. Schaerer, G. Meynet, and A. Maeder, 1992, Astron. Astrophys. 96, 269.

Schatz, H., A. Aprahamian, J. Görres, M. Wiescher, T. Rauscher, J. F. Rembges, F.-K. Thielemann, B. Pfeiffer, P. Möller, K.-L. Kratz, H. Herndl, B. A. Brown, and H. Rebel, 1997, Phys. Rep., in press.

Schmid, H. M., 1994, Astron. Astrophys. 284, 156.

Schmid, H. M., and H. Nussbaumer, 1993, Astron. Astrophys. 268, 159.

Schmidt, S., C. Rolfs, W. H. Schulte, H. P. Trautvetter, R. W. Kavanagh, C. Hategan, S. Faber, B. D. Valnion, and G. Graw, 1995, Nucl. Phys. A 591, 227.

Schönberg, M., and S. Chandrasekhar, 1942, Astrophys. J. 96, 161.

Schönberner, D., 1979, Astron. Astrophys. 79, 108.

Schönberner, D., 1981, Astron. Astrophys. 103, 119.

Schramm, D. N., 1982, in Essays in Nuclear Astrophysics, edited by C. A. Barnes, D. D. Clayton, and D. N. Schramm (Cambridge University Press, Cambridge), p. 325.

Schröder, U., H. W. Becker, G. Bogaert, J. Görres, C. Rolfs, and H. P. Trautvetter, 1987, Nucl. Phys. A 467, 240.

Schwarzschild, K., 1906, Göttinger Nachr. 41.

Schwarzschild, M., and R. Härm, 1965, Astrophys. J. 145, 496. Schwarzschild, M., and R. Härm, 1967, Astrophys. J. 150, 961. Seaton, M. J., Y. Yan, D. Mihalis, and A. K. Pradhan, 1994, Mon. Not. R. Astron. Soc. 266, 805.

Seeger, P. A., W. A. Fowler, and D. D. Clayton, 1965, Astrophys. J. Suppl. 97, 121.

Seuthe, S., C. Rolfs, U. Schröder, W. H. Schulte, E. Somorjai, H. P. Trautvetter, F. B. Waanders, R. W. Kavanagh, H. Ravn, M. Arnould, and G. Paulus, 1990, Nucl. Phys. A 514, 471.

Share, G. H., R. L. Kinzer, J. D. Kurfess, E. L. Chupp, and E. Rieger, 1985, Astrophys. J. 292, L61.

Shetrone, M. D., 1996a, Astron. J. 112, 1517.

Shetrone, M. D., 1996b, Astron. J. 112, 2639.

Shklovski, I. S., 1967, Astrophys. J. 148, L1.

Shukolyukov, A., and G. W. Lugmair, 1993, Science 259, 1138.

Smith, V. V., 1984, Astron. Astrophys. 132, 326.

Smith, V. V., K. Cunha, A. Jorissen, and H. Boffin, 1997, Astron. Astrophys., in press.

Smith, V. V., K. Cunha, A. Jorissen, and H. Boffin, 1996, Astron. Astrophys. 315, 179. 
Smith, V. V., and D. L. Lambert, 1984, Publ. Astron. Soc. Pac. 96, 226.

Smith, V. V., and D. L. Lambert, 1988, Astrophys. J. 333, 219. Smith, V. V., and D. L. Lambert, 1989, Astrophys. J. 345, L75. Smith, V. V., and D. L. Lambert, 1990, Astrophys. J. Suppl. Ser. 72, 387.

Smith, V. V., D. L. Lambert, and P. E. Nissen, 1993, Astrophys. J. 408, 262.

Smith, V. V., B. Plez, D. L. Lambert, and D. A. Lubowich, 1995, Astrophys. J. 441, 735.

Sneden, C., A. McWilliam, G. W. Preston, J. J. Cowan, D. L. Burris, and B. J. Armosky, 1996, Astrophys. J. 467, 819.

Sneden, C., and M. Parthasarathy, 1983, Astrophys. J. 267, 757.

Sneden, C., and C. A. Pilachowski, 1985, Astrophys. J. 288, L55.

Sneden, C., C. A. Pilachowski, and D. Vandenberg, 1986, Astrophys. J. 311, 826.

Spinka, H., and H. Winkler, 1972, Astrophys. J. 174, 455. Spinka, H., and H. Winkler, 1974, Nucl. Phys. A 233, 456.

Spite, F., P. Malliard, and M. Spite, 1984, Astron. Astrophys. 141, 56.

Spite, M., and F. Spite, 1978, Astron. Astrophys. 67, 23.

Spite, F., and M. Spite, 1982a, Astron. Astrophys. 115, 357.

Spite, F., and M. Spite, 1993, Astron. Astrophys. 279, L9.

Spite, M., P. Francois, P. E. Nissen, and F. Spite, 1996, Astron. Astrophys. 307, 172.

Spite, M., and F. Spite, 1982b, Nature (London) 297, 483.

Starrfield, S., W. Sparks, and J. W. Truran, 1974, Astrophys. J. Suppl. Ser. 28, 247.

Starrfield, S., W. Sparks, and J. W. Truran, 1978, Astrophys. J. 226, 168.

Steenbock, W., and H. Holweger, 1984, Astron. Astrophys. 130, 319.

Stegmüller, F., C. Rolfs, S. Schmidt, W. H. Schulte, H. P. Trautvetter, and R. W. Kavanagh, 1996, Nucl. Phys. A 601, 168.

Steigman, G., and T. P. Walker, 1992, Astrophys. J. 385, L13. Stephens, A., A. M. Boesgaard, J. K. King, and C. P. Deliyannis, 1997, Astrophys. J., submitted.

Stothers, R. B., and C.-W. Chin, 1992, Astrophys. J. 390, 136. Stothers, R. B., and C.-W. Chin, 1993a, Astrophys. J. 408, L85. Stothers, R. B., and C.-W. Chin, 1993b, Astrophys. J. 412, 294. Stothers, R. B., and C.-W. Chin, 1996, Astrophys. J. 468, 842. Straniero, O., R. Gallino, M. Busso, A. Chieffi, C. M. Raiteri, M. Limongi, and M. Salaris, 1995, Astrophys. J. 440, L85.

Strieder, F., L. Gialanella, U. Greife, C. Rolfs, S. Schmidt, W. H. Schulte, H. P. Trautvetter, D. Zahnow, F. Terrasi, L. Campajola, A. D'Onofrio, V. Roca, M. Romano, and M. Romoli, 1996, Z. Phys. A 355, 209.

Strohmayer, T. E., W. Zhang, J. H. Swank, A. Smale, L. Titarchuk, C. Day, and V. Lee, 1996, Astrophys. J. 469, L9.

Suess, H. E., and H. C. Urey, 1957, Rev. Mod. Phys. 28, 53.

Suntzeff, N. B., M. M. Phillips, J. H. Elias, D. L. DePoy, and A. R. Walker, 1992, Astrophys. J. Lett. 384, L33.

Sweigert, A. V., L. Greggio, and A. Renzini, 1989, Astrophys. J. Suppl. Ser. 69, 911.

Taam, R. E., 1983a, Astrophys. J. 268, 361.

Taam, R. E., 1983b, Astrophys. J. 270, 694.

Takahashi, K., H.-Th. Janka, J. Witti, 1994, Astron. Astrophys. 286, 857.

Takahashi, K., and K. Yokoi, 1987, At. Data Nucl. Data Tables 36, 375.

Takeda, Y., and M. Takada-Hidai, 1994, Publ. Astron. Soc. Jpn. 46, 395.
Tanaka, Y., and W. H. G. Lewin, 1995, in X-Ray Binaries, edited by W. H. G. Lewin, J. van Paradijs, and E. P. J. van den Heuvel (Cambridge University Press, Cambridge), p. 126.

Tayler, R. J., 1995, Mon. Not. R. Astron. Soc. 273, 215.

Taylor, R., 1990, Q. J. R. Astron. Soc. 31, 281.

Teegarden, B. J., et al., 1989, Nature (London) 339, 122.

The, L.-S., M. D. Leising, J. D. Kurfess, W. N. Johnson, D. H. Harmann, N. Gehrels, J. E. Grove, and W. R. Purchell, 1996, Astron. Astrophys. 120, C357.

Thielemann, F.-K., M. Hashimoto, and K. Nomoto, 1986, Astron. Astrophys. 158, 17.

Thielemann, F.-K., M. Hashimoto, and K. Nomoto, 1990, Astrophys. J. 349, 222.

Thielemann, F.-K., K.-L. Kratz, B. Pfeiffer, T. Rauscher, L. van Wromer, and M. C. Wiescher, 1994, Nucl. Phys. A 570, $329 \mathrm{c}$.

Thielemann, F.-K., K. Nomoto, and M. Hashimoto, 1996, Astrophys. J. 460, 408.

Thielemann, F.-K. K. Nomoto, M. Hashimoto, K. Iwamoto, and F. Brachwitz, 1997, Thermonuclear Supernovea, edited by P. Ruiz-Lapuente, R. Canal, and J. Isern (Kluwer, Dordrecht).

Thomas, D., D. N. Schramm, K. Olive, and B. Fields, 1993, Astrophys. J. 406, 569.

Thomas, D., D. N. Schramm, K. A. Olive, G.J. Mathews, B. S. Meyer, and B. D. Fields, 1994, Astrophys. J. 430, 291.

Thomas, J., Y. T. Chen, S. Hinds, D. Meredith, and M. Olson, 1986, Phys. Rev. C 33, 1679.

Thorburn, J. A., 1994, Astrophys. J. 421, 318.

Thorburn, J. A., and L. M. Hobbs, 1996, Astron. J. 111, 2106.

Thorburn, J. A., L. M. Hobbs, C. P. Deliyannis, and M. Pinsonneault, 1983, Astrophys. J. 415, 150.

Thorsett, S. E., Z. Arzoumanian, M. McKinnon, and J. H. Taylor, 1993, Astrophys. J. 405, L29.

Tilley, D. R., H. R. Weller, and C. M. Cheves, 1993, Nucl. Phys. A 564, 1.

Timmermann, R., H. W. Becker, C. Rolfs, U. Schröder, and H. P. Trautvetter, 1988, Nucl. Phys. A 477, 105.

Timmes, F. X., S. E. Woosley, and T. A. Weaver, 1995, Astrophys. J. Suppl. Ser. 98, 617.

Timmes, F. X., S. E. Woosley, and T. A. Weaver, 1996, Astrophys. J. 457, 834.

Tombrello, T. A., and P. D. Parker, 1963, Phys. Rev. 131, 2582.

Tomkin, J., and D. L. Lambert, 1979, Astrophys. J. 227, 209.

Tomkin, J., and D. L. Lambert, 1983, Astrophys. J. 273, 722.

Tomkin, J., and D. L. Lambert, 1986, Astrophys. J. 311, 819.

Tomkin, J., D. L. Lambert, B. Edvardsson, B. Gustafsson, and P. E. Nissen, 1989, Astron. Astrophys. Lett. 219, 15.

Tomkin, J., V. M. Woolf, D. L. Lambert, and M. Lemke, 1995, Astron. J. 109, 2204.

Toukan, K. A., K. Debus, F. Käppler, and G. Reffo, 1995, Phys. Rev. C 51, 1540.

Trautvetter, H. P., 1975, Nucl. Phys. A 243, 37.

Trautvetter, H. P., and C. Rolfs, 1975, Nucl. Phys. A 242, 519.

Trautvetter, H. P., G. Roters, C. Rolfs, S. Schmidt, and P. Descouvemont, 1996, in Proceedings of Nuclei in the Cosmos IV (Nucl. Phys. A 621, 161c).

Trimble, V., 1975, Rev. Mod. Phys. 47, 877.

Trimble, V., 1982, Observatory 102, 133.

Trimble, V., 1991, Astron. Astrophys. Rev. 3, 1. 
Trimble, V., 1995, in Cosmic Abundances, edited by S. E. Holt and G. Sonneborn, Astron. Soc. of the Pac. Conference Series, 99.

Truran, J. W., 1981, Astron. Astrophys. 97, 391.

Truran, J. W., 1996, private communication.

Truran, J. W., W. D. Arnett, and A. G. W. Camerson, 1967, Can. J. Phys. 45, 2315.

Truran, J. W., and A. G. W. Cameron, 1972, Astrophys. J. 171, 89.

Truran, J. W., C. J. Hansen, A. G. W. Cameron, and A. Gilbert, 1966, Can. J. Phys. 44, 151.

Truran, J. W., and I. Iben, Jr., 1977, Astrophys. J. 216, 797.

Tuli, J. K., 1995, Nuclear Wallet Cards, U.S. Nuclear Data Center.

Tutukov, A. V., and L. R. Yungelson, 1973, Nauch. Inf. 27, 57 (Russian).

Tutukov, A. V., and L. R. Yungelson, 1979, Acta Astron. 29, 665.

Tutukov, A. V., and L. R. Yungelson, 1992, Sov. Astron. 36, 266.

Tutukov, A. V., and L. R. Yungelson, 1993, Astron. Rep. 37, 411.

Tutukov, A. V., L. R. Yungelson, and I. Iben, Jr., 1992, Astrophys. J. 386, 197.

Utsumi, K., 1967, Publ. Astron. Soc. Jpn. 19, 342.

Utsumi, K., 1970, Publ. Astron. Soc. Jpn. 22, 92.

Utsumi, K., 1985, in Cool Stars with Excesses of Heavy Elements, edited by M. Jashek and P. C. Keenan (Reidel, Dordrecht), p. 243.

Uus, U., 1970, Nauch. Inform. Acad. Nauk. 17, 3.

Vandenberg, D. A., P. B. Stetson, and M. Bolte, 1996, Annu. Rev. Astron. Astrophys. 34, 461.

van den Bergh, S., R. D. McClure, and R. Evans, 1987, Astrophys. J. 323, 44.

van den Bergh, S., and G. A. Tammann, 1991, Annu. Rev. Astron. Astrophys. 29, 363.

van den Heuvel, E. P. J., 1985, in Evolution of Galactic X-Ray Binaries, edited by J. Truemper, W. H. G. Lewin, and W. Brinkman (Reidel, Dordrecht).

van den Heuvel, E. P. J., D. Bhattacharia, K. Nomoto, and S. Rappaport, 1992, Astron. Astrophys. 262, 97.

van den Heuvel, E. P. J., and J. Heise, 1972, Nature (London) 239, 67.

Vangioni-Flam, E., M. Cassé, B. D. Fields, and K. Olive, 1996, Astrophys. J. 468, 199.

Vangioni-Flam, E., M. Cassé, and R. Oberto, 1990, Astrophys. J. 349, 510 .

van Paradijs, J., 1995, in X-Ray Binaries, edited by W. H. G. Lewin, J. van Paradijs, and E. P. J. van den Heuvel (Cambridge University Press, Cambridge), p. 536.

van Paradijs, J., and N. White, 1995, Astrophys. J. 447, L33.

Vanture, A. D., 1992a, Astron. J. 103, 2035.

Vanture, A. D., 1992b, Astron. J. 104, 1997.

Vanture, A. D., G. Wallerstein, and J. A. Brown, 1994, Publ. Astron. Soc. Pac. 106, 835.

van Wormer, L., J. Görres, C. Iliadis, M. Wiescher, and F.-K. Thielemann, 1994, Astrophys. J. 432, 326.

Verbunt, F., and C. Zwann, 1981, Astron. Astrophys. 100, L7. Vogelaar, R. B., 1989, Ph.D. thesis, California Institute of Technology.

Vogelaar, R. B., L. W. Mitchell, R. W. Kavanagh, A. E. Champagne, P. V. Magnus, M. S. Smith, A. J. Howard, P. D. Parker, and H. A. O’Brien, 1996, Phys. Rev. C 53, 1945.
Vogt, E., 1968, in Advances in Nuclear Physics, Vol. 1, edited by M. Baranger and E. Vogt (Plenum, New York), p. 261.

Volk, H., H. Kräwinkel, R. Santo, and L. Wallek, 1983, Z. Phys. A 310, 91.

Volkening, J., and D. A. Papanastassiou, 1990, Astrophys. J. Lett. 258, L29.

von Ballmoos, P., R. Diehl, and V. Schönfelder, 1987, Astrophys. J. 318, 654.

von Weizsäcker, C. F., 1935, Z. Phys. 96, 431.

Voss, F., K. Wisshak, K. Guber, F. Käppeler, and G. Reffo, 1994, Phys. Rev. C 50, 2582.

Wagoner, R. V., 1973, Astrophys. J. 179, 343.

Wagoner, R. V., W. A. Fowler, and F. Hoyle, 1967, Astrophys. J. 148, 3 .

Walborn, N. R., 1987, in Atmospheric Diagnostics of Stellar Evolution: Chemical Peculiarity, Mass Loss and Explosion, edited by K. Nomoto, Lecture Notes in Physics No. 305 (Springer-Verlag, Berlin), p. 70.

Walker, T. P., G. Steigman, D. N. Schramm, K. A. Olive, and H.-S. Kang, 1991, Astrophys. J. 376, 51.

Wallace, R. K., and S. E. Woosley, 1981, Astrophys. J. Suppl. Ser. 45, 389.

Wallerstein, G., 1992, Publ. Astron. Soc. Pac. 104, 511.

Wallerstein, G., 1994, private communication.

Wallerstein, G., and P. S. Conti, 1996, Annu. Rev. Astron. Astrophys. 7, 99.

Wallerstein, G., J. L. Greenstein, R. Parker, H. L. Helfer, and L. H. Aller, 1963, Astrophys. J. 137, 280.

Wallerstein, G., A. D. Vanture, E. B. Jenkins, and G. Fuller, 1995, Astrophys. J. 449, 688.

Ward, R. A., and W. A. Fowler, 1980, Astrophys. J. 238, 266.

Warner, B., 1965, Mon. Not. R. Astron. Soc. 129, 163.

Warner, B., 1995, Cataclysmic Variable Stars (Cambridge University Press, Cambridge).

Warren, S. J., M. J. Irwin, D. W. Evans, J. Liebert, P. S. Osmer, and P. C. Hewett, 1993, Mon. Not. R. Astron. Soc. 261, 185.

Wasserburg, G. J., A. I. Boothroyd, and I.-J. Sackmann, 1995, Astrophys. J. 447, L37.

Weaver, T. A., and S. E. Woosley, 1993, Phys. Rep. 227, 65.

Webbink, R. F., 1984, Astrophys. J. 277, 355.

Webbink, R. F., and I. Iben, Jr., 1987, in The Second Conference on Faint Blue Stars, edited by A. G. Davis Philip, D. S. Hayes, and J. W. Liebert (Davis, Schenectady), p. 445.

Webbink, R. F., S. A. Rappaport, and G. J. Savonije, 1983, Astrophys. J. 270, 678.

Webbink, R. F., and V. Kalogera, 1994, in The Evolution of $X$-Ray Binaries, edited by S. S. Holt and C. S. Day (AIP, New York), p. 321.

Weigert, A., 1966, Z. Astrophys. 64, 395.

Weinberg, S., 1967, Phys. Rev. Lett. 19, 1264.

Wheeler, J. C., R. P. Harkness, A. M. Khokhlov, and P. Höflich, 1995, Phys. Rep. 256, 211.

Wheeler, J. C., C. Sneden, and J. W. Truran, Jr., 1989, Annu. Rev. Astron. Astrophys. 27, 279.

Wiescher, M., 1996, private communication.

Wiescher, M., J. Görres, B. Sherrill, M. Mohar, J. S. Winfield, and B. A. Brown, 1988, Nucl. Phys. A 484, 90.

Wiescher, M., J. Görres, F.-K. Thielemann, and H. Ritter, 1986, Astron. Astrophys. 160, 56.

Wiescher, M., and K. H. Langanke, 1986, Z. Phys. A 325, 309. Wiescher, M., R. Steininger, and F. Käppler, 1989, Astrophys. J. 344, 464. 
Winger, J. A., D. P. Bazin, W. Benenson, G. M. Crawley, D. J. Morrissey, N. A. Orr, R. Pfaff, B. M. Sherrill, M. Steiner, M. Thoennessen, S. J. Yannello, and B. M. Young, 1993, Phys. Lett. B 299, 24.

Winters, R., F. Käppeler, K. Wisshak, A. Mengoni, and G. Reffo, 1986, Astrophys. J. 300, 41.

Wisshak, K., K. Guber, F. Käppeler, J. Krisch, H. Müller, G. Rupp, and V. Voss, 1990, Nucl. Instrum. Methods Phys. Res. A 292, 595 .

Wisshak, K., K. Guber, F. Voss, F. Käppeler, and G. Reffo, 1993, Phys. Rev. C 48, 1401.

Wisshak, K., F. Voss, F. Käppeler, K. Guber, L. Kazakov, N. Kornilov, M. Uhl, and G. Reffo, 1995, Phys. Rev. C 52, 2762.

Witti, J., H.-Th. Janka, and K. Takahashi, 1994, Astron. Astrophys. 286, 841.

Wolfenstein, L., 1978, Phys. Rev. D 17, 2369.

Woolf, V. M., J. Tomkin, and D. L. Lambert, 1995, Astrophys. J. 453, 660 .

Wood, P. R., M. S. Bessell, and M. W. Fox, 1983, Astrophys. J. 272, 99.

Woosley, S. E., 1986, in Nucleoysnthesis and Chemical Evolution, edited by B. Hauk, A. Meader, and G. Meynet (Geneva Observatory, CH-1290 Sauverny-Versoix, Switzerland).

Woosley, S. E., 1996, Astrophys. J. 476, 801.

Woosley, S. E., W. D. Arnett, and D. D. Clayton, 1972, Astrophys. J. 175, 731.

Woosley, S. E., W. D. Arnett, and D. D. Clayton, 1973, Astrophys. J. Suppl. 26, 231.

Woosley, S. E., T. S. Axelrod, and T. A. Weaver, 1981, Comments Nucl. Part. Phys. 9, 185.

Woosley, S. E., D. H. Hartmann, R. D. Hoffman, and W. C. Haxton, 1990, Astrophys. J. 356, 272.

Woosley, S. E., and R. D. Hoffman, 1991, Astrophys. J. 368, L31.

Woosley, S. E., and R. D. Hoffman, 1992, Astrophys. J. 395, 202.

Woosley, S. E., and W. M. Howard, 1978, Astrophys. J. Suppl. 36, 285.

Woosley, S. E., and W. M. Howard, 1990, Astrophys. J. 354, L21.
Woosley, S. E., P. A. Pinto, D. Hartmann, 1989, Astrophys. J. 346, 395.

Woosley, S. E., and T. A. Weaver, 1986, Annu. Rev. Astron. Astrophys. 24, 205.

Woosley, S. E., and T. A. Weaver, 1994, Astrophys. J. 423, 371.

Woosley, S. E., and T. A. Weaver, 1995, Astrophys. J. Suppl. Ser. 101, 181.

Woosley, S. E., T. A. Weaver, and R. D. Hoffman, 1995, in Nuclei in the Cosmos III, edited by M. Busso, C. M. Raiteri, and R. Gallino (AIP, New York), p. 462.

Woosley, S. E., J. R. Wilson, G. H. Mathews, R. D. Hoffman, and B. S. Meyer, 1994, Astrophys. J. 433, 229.

Wu, S.-C., and C. A. Barnes, 1984, Nucl. Phys. A 422, 373.

Yorke, H. W., P. Bodenheimer, and R. E. Taam, 1995, Astrophys. J. 451, 308.

Yungelson, L. R., and A. V. Tutukov, 1991, in Wolf-Rayet Stars and Interactions with Other Massive Stars in Galaxies, edited by K. A. van den Hucht and B. Hidayat (Reidel, Dordrecht), p. 459.

Yungelson, L. R., and A. V. Tutukov, 1993, in Planetary Nebulae, edited by R. Weinberger and A. Acker (Kluwer, Dordrecht), p. 389.

Zappala, R. R., 1972, Astrophys. J. 172, 57.

Zhai, M., and D. M. Shaw, 1994, Meteoritics 29, 607.

Zhao, G., and P. Magain, 1990, Astron. Astrophys. 238, 242.

Zhao, G., and P. Magain, 1991, Astron. Astrophys. 244, 425.

Zhao, Z., E. Adelberger, and L. Debrackeler, 1995, unpublished.

Zhao, Z., R. H. France III, K. S. Lai, S. L. Rugari, M. Gai, and E. L. Wilds, 1993, Phys. Rev. Lett. 70, 2066.

Zucker, D. B., G. Wallerstein, and J. A. Brown, 1996, Publ. Astron. Soc. Pac. 108, 911.

Zuckerman, B., and L. H. Aller, 1986, Astrophys. J. 301, 772. Zyskind, J. L., R. Marrs, M. H. Shapiro, J. Davidson, C. A. Barnes, and W. A. Fowler, 1977, Bull. Am. Phys. Soc. 22, 542. Zyskind, J. L., R. Marrs, M. H. Shapiro, J. Davidson, C. A. Barnes, and W. A. Fowler, 1980, Nucl. Phys. A 343, 295.

Zyskind, J. L., M. Rios, and C. Rolfs, 1981, Astrophys. J. 243, L53; 245, L97. 